\title{
The Role of Nanoscale Atomic Fluctuations in the Superconducting Mechanism of the $\mathrm{BiS}_{2}$ Superconductors
}

\author{
Athauda Mudiyanselage Shalani Anushika K Athauda \\ Colombo, Sri Lanka
}

B.Sc., University of Colombo, Sri Lanka, 2010

A Dissertation presented to the Graduate Faculty of the University of Virginia in Candidacy for the Degree of Doctor of Philosophy

Department of Physics

University of Virginia

December, 2017 



\section{Abstract}

The interplay of disorder and superconductivity has been a topic of intense interest for decades. Several exemplary systems can be found in the literature that demonstrate how disorder, manifested through doping, vacancies and strain in both two and three dimensions, plays an important role in the pairing mechanism. In the cuprates for example, it is well understood by now that disorder induced by doping leads to charge puddles creating an inhomogeneous lattice, and superconductivity is the result of percolation through filamentary regions surrounded by the puddles.

The newly discovered Bismuth Sulfide superconductors provide a testbed for investigating the effects of disorder on superconductivity in the absence of magnetism. The superconducting $\mathrm{BiS}_{2}$ planes consist of the same building blocks as in the cuprate and Febased superconductors. Charge carriers are introduced either via doping in the blocking layers or by introducing vacancies. The pairing mechanism is electron-phonon mediated, possibly of the conventional type but with strong electronic correlations indicated by electron phonon coupling constant of $\lambda \sim 0.8$. Moreover, they have been theoretically predicted to have unstable phonon modes, a charge density wave (CDW) instability, and strong spin orbit coupling.

In this dissertation, the characteristic nature of the lattice distortions, the effects of atomic inhomogeneities and how they couple to the mechanism of superconductivity are investigated in the $\mathrm{BiS}_{2}$ class. By combining neutron and high resolution X-ray diffraction, the structural investigation is carried out on polycrystalline and single crystal samples of $\mathrm{LnO}_{1-x} \mathrm{~F}_{x} \mathrm{BiS}_{2}(\mathrm{Ln}=\mathrm{La}, \mathrm{Nd}$ and $\mathrm{Pr})$. 
While on average, the crystal symmetry is presumed to be tetragonal, our results provide evidence for charge disproportionation of the Bi ions linked to a locally distorted lattice. In-plane ferro lattice distortions are evident from the local displacements of sulfur and bismuth in the basic building blocks giving rise to superlattice reflections. The inplane short-range distortions break the 2-fold and 4-fold symmetry operations and create distinct environments around $\mathrm{Bi}$. The ferro-distortive sulfur displacements occur in both non-superconducting and superconducting phases with diminished magnitude of displacements in the latter. In the superconducting phase, the out-of-plane motion of apical sulfur becomes significant that can act as charge transfer between the donor layers and superconducting layers. The lattice distortions that arise from these local modes may trap charge carriers decreasing the number of pairing electrons. Thus, the work presented in this thesis provides insight into the structural distortions coupled to the charge fluctuations and carrier transfer in $\mathrm{BiS}_{2}$ superconductors. 


\section{Acknowledgements}

First and foremost, it is with great pleasure I express my deeply felt gratitude to my advisor Prof. Despina Louca for her constant encouragement, clear guidance, invaluable feedback and constructive suggestions I have received throughout my $\mathrm{PhD}$. I owe her a deep debt of gratitude for all the opportunities I was given to attend conferences, and participate in experiments at national laboratories. My hearfelt thanks go to her for all the support and timely advice extended to me in diverse situations in all these years which is not limited to work.

I would like to express my deeply felt gratitude to the other members of my thesis committee, Prof. Israel Klich, Prof. Gia-Wei Chern and Prof. Leonid Zhigilei, and my research committee member, Prof. Jongsoo Yoon.

I owe a deep debt of gratitude to Prof. Nilanga Liyanage for all the advise and support given to me and being a great strength in numerous situations throughout my PhD.

I must thank the instrument scientists at the neutron and x-ray facilities; Dr. Christina Hoffman, Dr. Mikhail Feygenson and Dr. Joerg Neuefeind at Oak Ridge National Laboratory and Dr. Yang Ren, Dr. Przemyslaw Dera and Dr. Dongzhou Zhang at Argonne National Laboratory for their collaboration, useful discussions and support. I must thank Dr. Yoshikazu Mizuguchi's group, Tokyo Metropolitan University, Japan, Dr. Masanori Nagao's group, University of Yamanashi, Japan, and Dr. Gang Cao's group, University of Kentucky, for providing samples and their collaboration.

I would like to thank Dr. Junjie Yang, Dr. Bing Li, Dr. Shinichiro Yano and Dr. Keeseong Park for teaching me prepare samples and use x-ray diffractometor and physical property measurement system, and for all the useful discussions. I am thankful to 
my fellow lab mates, Chunruo Duan and Aaron Wegner for all the discussions and fun we shared.

I would like to call my friends to mind with sincere gratitude, especially, Kapila Wijayaratne, Udari Jayasiri, Harshanee Jayasekara and Amali Premathilake for the immense support and strength given to me in a number of situations.

Finally, I would like to thank my family for standing there as pillars of strength in my life. I am immensely grateful to my husband, Sachith Dissanayake, for all the patience, support, love and care, and my little daughter, Sanuki, for her sweet words and cute smiles. 


\section{Contents}

$\begin{array}{ll}\text { Abstract } & \text { ii }\end{array}$

Acknowledgements iv

Contents vi

List of Figures $\quad$ ix

List of Tables $\quad$ xiii

1 Introduction $\quad 1$

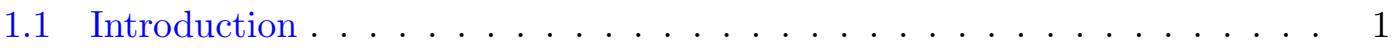

1.2 Layout of the Dissertation . . . . . . . . . . . . . . . 8

2 Background $\quad 10$

2.1 Introduction to $\mathrm{BiS}_{2}$ Superconductors . . . . . . . . . . . . . . . 10

2.2 High pressure-induced superconductivity in $\mathrm{BiS}_{2}$ superconductors . . . . . 12

2.3 Charge density wave instability in $\mathrm{BiS}_{2}$ superconductors . . . . . . . . . . 15

2.4 Hidden spin polarization in $\mathrm{BiS}_{2}$ superconductors . . . . . . . . . . . . . 16

2.5 Theoretically predicted possible symmetry lowering in $\mathrm{BiS}_{2}$ superconductors 17

2.6 Suggested superconducting mechanisms for $\mathrm{BiS}_{2}$ superconductors . . . . 20

2.6.1 Possible conventional pairing . . . . . . . . . . . . . . . . . . 20

2.6.2 Possible unconventional pairing . . . . . . . . . . . . . . . . . . . 21

2.7 Suggested possible pairing symmetries . . . . . . . . . . . . 24

3 Neutron and Synchrotron X-ray Scattering $\quad \mathbf{2 7}$

3.1 Introduction to Neutron Scattering . . . . . . . . . . . . . . . . . 27

3.2 Neutron Scattering Theory _ . . . . . . . . . . . . . . . . . 29

3.2 .1 Scattering geometry . . . . . . . . . . . . . . . . 29

3.2 .2 Nuclear scattering cross-section . . . . . . . . . . . . . . . . . . . . . . . . . . . . . . . . . .

3.2 .3 Bragg diffraction . . . . . . . . . . . . . . . . 33

3.3 Pair Distribution Function . . . . . . . . . . . . . . . . . . 35

3.4 Synchrotron X-ray scattering . . . . . . . . . . . . . . 40

3.5 Neutron scattering instruments . . . . . . . . . . . . . . . . . . 41 
3.5.1 Neutron powder diffractometer (NPD) . . . . . . . . . . . . 41

3.5.2 Time of flight Laue diffractometer . . . . . . . . . . . . . . . . . . . . . . . . . . 44

3.5.3 Four circle diffractometer . . . . . . . . . . . . . . . . 45

3.6 Data analysis . . . . . . . . . . . . . . . . . . . . . . . . . . . . . 45

3.6.1 Powder diffraction data analysis . . . . . . . . . . . . . . . . 45

3.6.2 Pair density function analysis and modelling . . . . . . . . . . . . . 47

3.6.3 Single crystal data analysis . . . . . . . . . . . . . . . . . . . 49

3.7 Sample preparation and characterization . . . . . . . . . . . 50

3.7.1 The transition temperature of $\mathrm{NdO}_{1-x} \mathrm{~F}_{x} \mathrm{BiS}_{2} \ldots \ldots \ldots$. . . . . 51

3.7.2 The transition temperature of $\mathrm{ReO}_{0.7} \mathrm{~F}_{0.3} \mathrm{BiS}_{2}(\mathrm{Re}=\mathrm{La}$ and $\mathrm{Nd})$ single crystals . . . . . . . . . . . . . . . . 52

4 The crystal structure and nature of local lattice distortions in superconducting $\mathrm{LaO}_{1-x} \mathrm{~F}_{x} \mathrm{BiS}_{2} \quad \mathbf{5 4}$

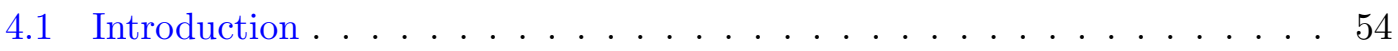

4.2 Crystal structure of $\mathrm{LaO}_{0.5} \mathrm{~F}_{0.5} \mathrm{BiS}_{2} \ldots \ldots \ldots \ldots \ldots \ldots$

4.3 The Debye temperature of $\mathrm{LaO}_{1-x} \mathrm{~F}_{x} \mathrm{BiS}_{2} \ldots \ldots \ldots \ldots$. . . . . . . . 65

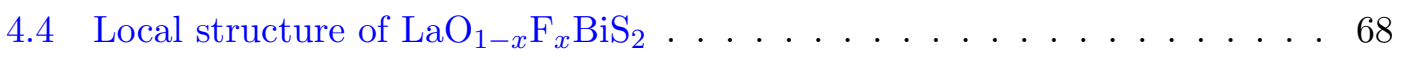

4.4.1 Composition dependence of local structure . . . . . . . . . . 68

4.4 Lattice distortions of $\mathrm{LaOBiS}_{2} \ldots \ldots \ldots$. . . . . . . . . . 70

4.4.2.1 Nature of lattice distortions in $\mathrm{LaOBiS}_{2} \ldots \ldots$. . . . . 71

4.4.3 Lattice distortions in $\mathrm{F}$ doped $\mathrm{LaOBiS}_{2}$. . . . . . . . . . . . . . . 75

4.4.4 Temperature dependence of bond length splitting . . . . . . . . . 78

4.5 Summary . . . . . . . . . . . . . . . . . . . . 80

5 Crystal structure and local lattice distortions in $\mathrm{NdO}_{1-x} \mathrm{~F}_{x} \mathrm{BiS}_{2}$ superconductor $\quad \mathbf{8 2}$

5.1 Introduction . . . . . . . . . . . . . . . . 82

5.2 Crystal structure of $\mathrm{NdO}_{1-x} \mathrm{~F}_{x} \mathrm{BiS}_{2} \ldots \ldots \ldots \ldots \ldots$. . . . . . . 86

5.3 The local structure of $\mathrm{NdO}_{1-x} \mathrm{~F}_{x} \mathrm{BiS}_{2} \ldots \ldots \ldots \ldots$. . . . . . . . . . . . . . . . 94

5.3.1 Composition dependence of local structure . . . . . . . . . . . 94

5.3.2 Nature of lattice distortions in $\mathrm{NdO}_{0.8} \mathrm{~F}_{0.2} \mathrm{BiS}_{2} \ldots \ldots \ldots$. . . . . . 95

5.3.3 Nature of lattice distortions in $\mathrm{NdO}_{0.6} \mathrm{~F}_{0.4} \mathrm{BiS}_{2} \ldots \ldots$. . . . . . . . 99

5.3.4 Temperature dependence of bond length splitting . . . . . . . . . 103

5.4 Summary . . . . . . . . . . . . . . . . . . . 103

6 Ferro-lattice-distortions and charge fluctuations in superconducting $\mathbf{R e O}_{1-x} \mathbf{F}_{x} \mathrm{BiS}_{2}(\mathrm{Re}=\mathrm{La}, \mathrm{Nd}$ and Pr) 105

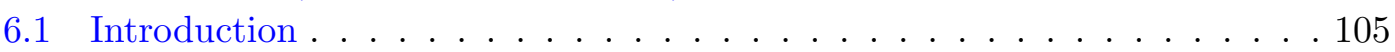

6.2 Symmetry lowering in $\mathrm{LaOBiS}_{2} \ldots$. . . . . . . . . . . . . . . 107

6.2.1 Testing the theoretically predicted symmetries . . . . . . . . 109

6.2.2 Sulfur distortions as a possible candidate . . . . . . . . . . . 112

6.2.3 Crystal structure refinement using $(+\mathrm{x},+\mathrm{y})$ displacement mode .115

6.2.4 Stacking of sulfur displacement modes as a candidate . . . . . . 118

6.3 Diffraction patterns of $\mathrm{ReO}_{0.7} \mathrm{~F}_{0.3} \mathrm{BiS}_{2} \ldots \ldots \ldots \ldots . \ldots \ldots$

6.4 Strain effect on $\mathrm{T}_{c}$ of $\mathrm{NdO}_{0.7} \mathrm{~F}_{0.3} \mathrm{BiS}_{2}$ single crystals $\ldots \ldots \ldots \ldots . . \ldots 121$

6.4.1 Characterization of strain of piezo stack . . . . . . . . . . 122 
6.4.2 Resistance of $\mathrm{NdO}_{0.7} \mathrm{~F}_{0.3} \mathrm{BiS}_{2}$ single crystals under strain $\ldots . .126$

6.5 Summary . . . . . . . . . . . . . . . . . . . . . 128

$\begin{array}{llr}7 & \text { Conclusions } & 129\end{array}$

$\begin{array}{ll}\text { Bibliography } & 133\end{array}$ 


\section{List of Figures}

1.1 The Cooper pair (green oval) formation of electrons (blue circles). The electron is attracted to the positive charge density (pink glow) created by the first electron around itself by distorting the lattice. . . . . . . . . . . 3

1.2 The anion height dependence of $\mathrm{T}_{c}$ in Fe-based superconductors. The curve peaks at $\sim 1.38 \AA \ldots \ldots \ldots \ldots$

1.3 The $\mathrm{T}_{c}$ vs AsFeAs bond angle for various pnictide superconductors. The AsFeAs bond angle determines the distortion of the Fe tetrahedron. Formulas of parent compositions of superconductors are shown in the inset. Crystal structure parameters of the samples are selected at the maximum $\mathrm{T}_{c}$ of each system. The vertical dashed line indicates the bond angle of a regular tetrahedron. . . . . . . . . . . . . . .

2.1 Schematic diagrams of layered crystal structures of $\mathrm{BiS}_{2}$ based superconductors, (a) $\mathrm{LaOBiS}_{2}$ (b) $\mathrm{SrFBiS}_{2}$ (c) $\mathrm{Eu}_{3} \mathrm{~F}_{4} \mathrm{Bi}_{2} \mathrm{~S}_{4}$ and (d) $\mathrm{Bi}_{6} \mathrm{O}_{4} \mathrm{~S}_{4}\left(\mathrm{SO}_{4}\right.$ ).

2.2 (a) magnetic susceptibility, (b) electrical resistivity and (c) specific heat measurements for the superconductor $\mathrm{Bi}_{4} \mathrm{O}_{4} \mathrm{~S}_{3}$ as a function of temperature. 11

2.3 The magnetic susceptibility of $\mathrm{LaO}_{0.5} \mathrm{~F}_{0} .5 \mathrm{BiS}_{2}$ as a function of temperature prepared under (a) ambient conditions (AP2) and (b) high pressure, HP. The high pressured samples are prepared using AP2 process and annealed secondarily with $2 \mathrm{GPa}$ at $600^{\circ} \mathrm{C} \ldots \ldots \ldots$

2.4 Phase diagrams of (a) $\mathrm{PrO}_{0.5} \mathrm{~F}_{0} .5 \mathrm{BiS}_{2}$ (b) $\mathrm{NdO}_{0.5} \mathrm{~F}_{0} .5 \mathrm{BiS}_{2}$ (c) $\mathrm{LaO}_{0.5} \mathrm{~F}_{0} .5 \mathrm{BiS}_{2}$

(d) $\mathrm{CeO}_{0.5} \mathrm{~F}_{0} .5 \mathrm{BiS}_{2}$ under pressure. The length of the vertical bars represent the transition width $\Delta \mathrm{T}_{c}$ and the bar caps represent the $\mathrm{T}_{c}{ }^{\text {onset }}$ (upper) and $\mathrm{T}_{0}$ (lower). The color shows the energy gap $\Delta$ in the semiconducting region where in (b) the green region to the right of the crosshatch corresponds to the metalization of $\mathrm{NdO}_{0.5} \mathrm{~F}_{0} .5 \mathrm{BiS}_{2}(\Delta=0) \ldots \ldots \ldots 14$

2.5 Phonon dispersion curves of $\mathrm{LaO}_{1-x} \mathrm{~F}_{x} \mathrm{BiS}_{2}$ at $\mathrm{x}=0$ and $\mathrm{x}=0.5$. . . . . 16

2.6 The sinusoidal atomic arrangement (top) and the one dimensional channels (bottom) of S and Bi atoms in the CDW phase. The pink and yellow spheres correspond to Bi and $\mathrm{S}$ atoms, respectively. . . . . . . . . 16

2.7 The unstable phonons of (a) $\mathrm{LaOBiS}_{2}$ and (b) $\mathrm{LaO}_{0.5} \mathrm{~F}_{0.5} \mathrm{BiS}_{2} \ldots \ldots$

2.8 Total energy during transformations to final all parameters relaxed polytypes $\mathrm{T}_{1}^{*}$ and $\mathrm{T}_{2}^{*}$. The red arrows show the direction of atomic displacement. 19

2.9 The resistivity of $\mathrm{NdO}_{1-x} \mathrm{~F}_{x} \mathrm{BiS}_{2}$ as a function of temperature under different magnetic fields. (a) $\mathrm{H} \| c$ and (b) $\mathrm{H} \| a b \ldots \ldots 22$

2.10 The resistivity of $\mathrm{NdO}_{0.5} \mathrm{~F}_{0.5} \mathrm{BiS}_{2}$ as a function of temperature at zero field. The inset shows the superconducting fluctuation up to $20 \mathrm{~K}$ and bulk superconductivity at $5 \mathrm{~K}$. 
2.11 The Fermi surface of the two orbital model. (a) Sign conserving $s$ wave for attractive pairing interactions such as mediated by phonons or charge fluctuations, (b) sign reversing $s$ wave, and (c) $d$ wave superconducting gaps obtained for repulsive pairing interactions mediated by spin fluctuations. . . . . . . . . . . . . . . . . . . 25

2.12 The intensity plots for imaginary parts of the spin susceptibility, Im $\chi(\mathrm{q}, \omega)=0.075$ for (a) $d$-wave, (b) extended $s$-wave, (c) $p$-wave $\chi^{+-}$and

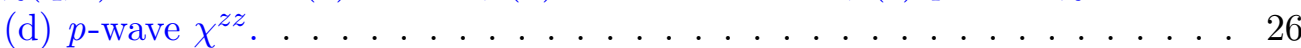

3.1 A cartoon illustrating how neutrons are produced by fission at reactor sources and by spallation at spallation sources. . . . . . . . . . . . 28

3.2 The scattering triangle with the incident wave vector $\vec{k}_{i}$, scattered wave vector $\vec{k}_{f}$ and transfered wave vector $\vec{Q}=\vec{k}_{i}-\vec{k}_{f} \ldots \ldots . \ldots 30$

3.3 The layout of the neutron powder diffractometer, D1B. . . . . . . . . . . . 42

3.4 The principle of time of flight technique. . . . . . . . . . . . . 43

3.5 Schematic diagram of Nanoscale-Ordered Materials Diffractometer . . . . 44

3.6 Topaz - high resolution Laue neutron single-crystal diffractometer located at Oak Ridge National Laboratory . . . . . . . . . . . . . . . . . . . . 45

3.7 The layout of a four circle diffractometer. . . . . . . . . . . . . . . 46

3.8 The susceptibility data of $\mathrm{NdO}_{1-x} \mathrm{~F}_{x} \mathrm{BiS}_{2}$ for non-superconducting $x=0.2$

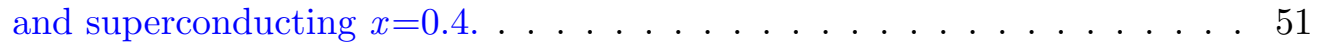

3.9 The bulk magnetic susceptibility of the $\mathrm{LaO}_{0.7} \mathrm{~F}_{0.3} \mathrm{BiS}_{2}$ crystal showing a

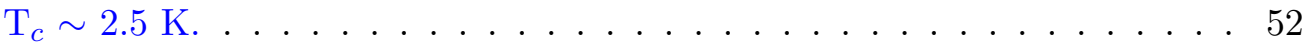

3.10 Resistivity data of $\mathrm{NdO}_{0.7} \mathrm{~F}_{0.3} \mathrm{BiS}_{2}$ crystal showing a $\mathrm{T}_{c} \sim 5 \mathrm{~K} . \ldots . .53$

4.1 The band structure of $\mathrm{LaOBiS}_{2} \ldots \ldots \ldots \ldots \ldots$. . . . . . . . . 55

4.2 Phase diagram of $\mathrm{LaO}_{1-x} \mathrm{~F}_{x} \mathrm{BiS}_{2}$ prepared under high pressure annealing. 56

$4.3 \mathrm{BiS}_{2}$ plane of $\mathrm{LaO}_{0.5} \mathrm{~F}_{0.5} \mathrm{BiS}_{2}$ at $\mathrm{x}=0$ and $0.5 \ldots \ldots \ldots \ldots$

4.4 Band structure of $\mathrm{LaO}_{0.5} \mathrm{~F}_{0.5} \mathrm{BiS}_{2}$ with and without spin orbit coupling. . 58

4.5 The neutron powder diffraction data of (a) $\mathrm{LaOBiS}_{2}$ at $6 \mathrm{~K}$, (b) $\mathrm{LaO}_{0.8} \mathrm{~F}_{0.2} \mathrm{BiS}_{2}$ at $2 \mathrm{~K}$, (c) $\mathrm{LaO}_{0.7} \mathrm{~F}_{0.3} \mathrm{BiS}_{2}$ at $2 \mathrm{~K}$, and (d) $\mathrm{LaO}_{0.5} \mathrm{~F}_{0.5} \mathrm{BiS}_{2}$ (HP annealed)

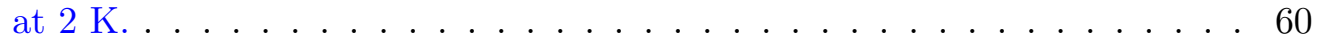

4.6 The comparison of (a) (110) (b) (114) Bragg peaks between the $x=0$

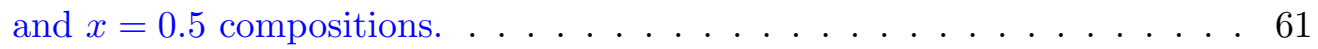

4.7 The gradual change of lattice constants with $\mathrm{F}$ doping. . . . . . . . . . . . 62

4.8 The unit cell of $\mathrm{LaO}_{1-x} \mathrm{~F}_{x} \mathrm{BiS}_{2}$ with the BiS tetrahedra. . . . . . . . . . 63

4.9 (a) The change of buckling angle (in degrees) with respect to a flat $\left(0^{\circ}\right)$ $\mathrm{BiS}_{2}$ plane (blue) and the bond length of $\mathrm{Bi}-\mathrm{S} 2$ change (red) as a function of doping level. . . . . . . . . . . . . . . . . 6 65

4.10 The temperature dependence of the pair density function of $\mathrm{LaOBiS}_{2}$. . . 66

4.11 The temperature dependence of squared FWHM values of the first peak of the local structural data of $\mathrm{LaOBiS}_{2}$, extracted from a Gaussian fitting. The fit of correlated Debye model is represented by the solid line. The error bars were calculated using one standard deviation of FWHM values of the Gaussian fitting . . . . . . . . . . . . . . . . . . . . 67

4.12 Composition dependence of the data corresponding to the local structures of $x=0,0.2,0.3$ and 0.5 . The data of $x=0$ were collected at $6 \mathrm{~K}$ and $x=0.2$, 0.3 and 0.5 were collected at $2 \mathrm{~K} \ldots \ldots \ldots \ldots$. . . . . . . . 69 
4.13 The PDF of $\mathrm{LaOBiS}_{2}$ at $6 \mathrm{~K}$ (black symbols) is compared to the PDF of average model (dashed blue line) . . . . . . . . . . . . . . . 71

4.14 (a) A schematic of the $\mathrm{BiS}_{2}$ plane showing the antiferrodistortive type mode. (b) A schematic of the $\mathrm{BiS}_{2}$ plane showing the ferrodistortive type mode. The displacements of $\mathrm{S} 1$ are either in the $\mathrm{x}$ or $\mathrm{y}$ direction. . . . . . 72

4.15 The two Bi-S1 planes in one unit cell of $\mathrm{LaO}_{1-x} \mathrm{~F}_{x} \mathrm{BiS}_{2}$ at $\mathrm{z} \sim \frac{1}{3}$ and $\mathrm{z} \sim \frac{2}{3}$ ). 73

4.16 Stripes of charge fluctuations in the two BiS1 planes of the crystal structure. 74

4.17 The PDF of $\mathrm{LaOBiS}_{2}$ at $6 \mathrm{~K}$ (black symbols) is compared to the PDF of average model (dashed blue line) and the local model (solid red line). . . . 75

4.18 The PDF of $\mathrm{LaOBiS}_{2}$ at $6 \mathrm{~K}$ (black symbols) is compared to the PDF of average model (dashed blue line) and the local model (solid red line). . . 76

4.19 The partial PDFs of Bi-S1 and Bi-S2 for $x=0$ (top) and $x=0.5$ (bottom). $\quad 77$

4.20 A schematic of the Bi-S tetrahedron with the bond lengths detremined from the refinement of the local structures of $x=0,0.2,0.3$ and $0.5 \ldots \ldots 78$

4.21 The temperature dependence the short (square) and long (circle) local Bi$\mathrm{S} 1$ bond lengths is contrasted to the Bi-S1 average bond length (triangle)

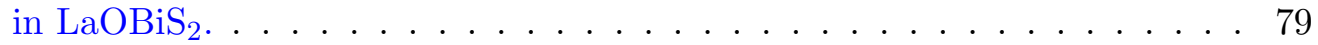

4.22 The temperature dependence of bond lengths in $\mathrm{LaO}_{1-x} \mathrm{~F}_{x} \mathrm{BiS}_{2}$. (a) The short (square) and long (circle) local Bi-S1 bond lengths is contrasted to the Bi-S1 average bond length (triangle). (b) The height of the S2 atom from the oxygen plane obtained from fitting the data of the local structure and from the Rietveld refinement of the average structure. . . . . . . . . 80

5.1 In-plane chemical pressure dependence of $\mathrm{T}_{c}$ for $\mathrm{ReO}_{1-x} \mathrm{~F}_{x} \mathrm{BiS}_{2}$ and $\mathrm{LaO}_{1-x} \mathrm{~F}_{x} \mathrm{Bi}\left(\mathrm{S}_{1-y} \mathrm{Se}_{y}\right)_{2} \ldots \ldots \ldots \ldots . \ldots \ldots$

5.2 The unit cell of $\mathrm{NdO}_{1-x} \mathrm{~F}_{x} \mathrm{BiS}_{2}$ with layers containing $\mathrm{BiS}_{2}$ polyhedra. . . 85

5.3 The neutron powder diffraction pattern of $\mathrm{NdO}_{1-x} \mathrm{~F}_{x} \mathrm{BiS}_{2}$ for (a) $x=0.2$

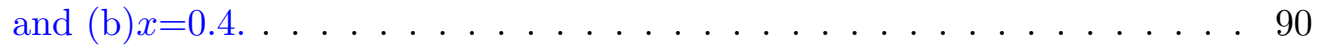

5.4 The lattice parameters (a) $a$ and (b) $b$, and (c) unit cell volume of $\mathrm{ReO}_{1-x} \mathrm{~F}_{x} \mathrm{BiS}_{2}$ for $\mathrm{Re}=\mathrm{Nd}$ and La. . . . . . . . . . . . . . . . . . 92

5.5 The isotropic thermal factors of $\mathrm{NdO}_{1-x} \mathrm{~F}_{x} \mathrm{BiS}_{2}$ is shown for (a) $x=0.2$ and $(\mathrm{b}) x=0.4 \ldots \ldots \ldots \ldots$

5.6 A comparison of the local structural data at $x=0.2$ and $x=0.4$ at $2 \mathrm{~K}$. . 94

5.7 The comparison of average model (in blue dashed line) and local model (in red solid line) with data represented by black dots in $\mathrm{NdO}_{0.8} \mathrm{~F}_{0.2} \mathrm{BiS}_{2} \quad 96$

5.8 The partial pair density function of Bi-S1 for $x=0.2$ in $\mathrm{NdO}_{1-x} \mathrm{~F}_{x} \mathrm{BiS}_{2}$. . 96

5.9 The Bi-S planes at $\mathrm{z} \sim 0.2 \mathrm{c}, 0.4 \mathrm{c}, 0.6 \mathrm{c}$ and $0.8 \mathrm{c} \ldots \ldots \ldots 7$

5.10 The schematics of $\mathrm{BiS}_{2}$ planes at $2 \mathrm{~K}$ depicting the $\mathrm{S} 1$ and $\mathrm{Bi}$ motion in $\mathrm{NdO}_{1-x} \mathrm{~F}_{x} \mathrm{BiS}_{2}$, two magnitudes of bond length at $x=0.2$. (b) Depicts the in-plane and out of plane displacements of $\mathrm{S}$ and $\mathrm{Bi}$ at $x=0.2 . \quad \ldots 98$

5.11 The comparison of average model (in blue dashed line) and local model (in red solid line) with data represented by black dots in $\mathrm{NdO}_{0.6} \mathrm{~F}_{0.4} \mathrm{BiS}_{2} .100$

5.12 The schematics of $\mathrm{BiS}_{2}$ planes at $2 \mathrm{~K}$ depicting the $\mathrm{S} 1$ and $\mathrm{Bi}$ motion in $\mathrm{NdO}_{1-x} \mathrm{~F}_{x} \mathrm{BiS}_{2}$, four magnitudes of bond length at $x=0.4$. (b) Depicts the in-plane and out of plane displacements of $\mathrm{S}$ and $\mathrm{Bi}$ at $x=0.4$. . . 101

5.13 The partial pair density function of Bi-S1 for $x=0.4$ in $\mathrm{NdO}_{1-x} \mathrm{~F}_{x} \mathrm{BiS}_{2}$. . 101 
5.14 The temperature dependence of bond length splitting of $\mathrm{NdO}_{1-x} \mathrm{~F}_{x} \mathrm{BiS}_{2}$

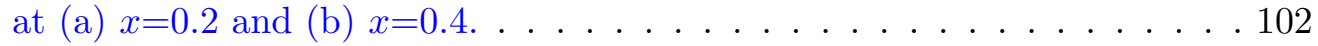

6.1 (a) The single crystal neutron diffraction pattern in the hk0 reciprocal plane for the parent compound, $\mathrm{LaOBiS}_{2}$. In (b) the $h k 0$ plane is constructed using the $\mathrm{P} 4 / \mathrm{nmm}$ symmetry. . . . . . . . . . . . . . 108

6.2 The integrated intensity of the observed peaks is plotted along the $h \overline{3} 0$ line.109

6.3 The calculated reciprocal patterns based on (a) $P 4 m m$, (b) $P \overline{4} m 2$, (c) $P 2_{1} m n$ and (d) $P 2_{1} / m$ symmetries. . . . . . . . . . . . . 110

6.4 Displacement modes of sulfur. In (a) the ferro-distortive and (b) the antiferro-distortive displacement modes are shown. . . . . . . . . . . . . 114

6.5 In (a) The calculated diffraction pattern from the ferro-distortive mode is shown while in (b) the diffraction pattern resulting from the antiferrodistortive mode is shown. . . . . . . . . . . . . . . . . . . 115

6.6 The single crystal refinement result. The square of the structure function, $|F(Q)|^{2}$, for the observed intensity is compared to the calculated intensity obtained from the refinement based on $(\mathrm{x}, \mathrm{y})$ mode. The fitting is done using the neutron single crystal data. The goodness of fit is determined to be 0.1148 . The blue line represents the ideal match between the calculated and the observed value at each Bragg peak with a slope of 1 . Deviations are observed for the highest intensity peaks. In the inset, the two $\mathrm{BiS}_{2}$ layers corresponding to displacements of $\mathrm{S} 1$ are shown. . . . . . . . . . 117

6.7 The calculated diffraction pattern is shown based on an alternating ( $\mathrm{x}, \mathrm{y})$ mode with (-x, -y) mode along c-axis. The circled are the extra reflections not present in data. In the side panel, the four Bi-S planes in the doubled unit cell along $c$ depicting the alternating $(\mathrm{x}, \mathrm{y})$ mode and $(-\mathrm{x},-\mathrm{y})$ mode is shown. . . . . . . . . . . . . . . . . . . . . . . 119

6.8 The synchrotron x-ray data in the $h k 0$ plane are shown for (a) $\mathrm{LaOBiS}_{2}$, (b) $\mathrm{LaO}_{0.7} \mathrm{~F}_{0.3} \mathrm{BiS}_{2}$, (c) $\mathrm{NdO}_{0.7} \mathrm{~F}_{0.3} \mathrm{BiS}_{2}$ and (d) $\mathrm{PrO}_{0.7} \mathrm{~F}_{0.3} \mathrm{BiS}_{2}$. The circled are the Bragg peaks when $h+k$ is odd ( $\mathrm{In} \mathrm{PrO}_{0.7} \mathrm{~F}_{0.3} \mathrm{BiS}_{2}$, they are not circled due to the poor Bragg peaks with powder streaks.). . . . . . 120

6.9 The photograph of the set up arranged for characterizing the piezo stack

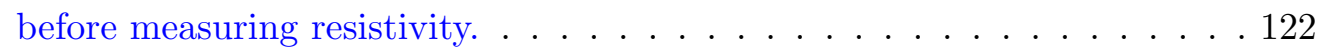

6.10 The definition of strain. . . . . . . . . . . . . . . . . . 123

6.11 Wheatstone bridge with the strain gauge as one resistive arm. . . . . . . 123

6.12 Output voltage of the bridge vs applied piezo voltage is shown where the output voltage, $V_{0}$ linearly increased negatively indicating an expansion. . 125

6.13 Strain vs.applied piezo voltage at room temperature. . . . . . . . . . . 126

6.14 Resistance of $\mathrm{NdO}_{0.7} \mathrm{~F}_{0.3} \mathrm{BiS}_{2}$ single crystals as a function of temperature, without strain $(0 \mathrm{~V})$ and with strain $(49 \mathrm{~V}) \ldots \ldots . \ldots . \ldots 127$ 


\section{List of Tables}

$2.1 \mathrm{~T}_{c}$ values for $\mathrm{ReO}_{0.5} \mathrm{~F}_{0} .5 \mathrm{BiS}_{2}(\mathrm{Re}=\mathrm{La}, \mathrm{Ce}, \mathrm{Pr}, \mathrm{Nd}) \ldots \ldots \ldots \ldots \ldots$

3.1 Fundamental properties of neutron . . . . . . . . . . . . . . . 29

4.1 A list of the crystal structure parameters of $\mathrm{LaO}_{1-x} \mathrm{~F}_{x} \mathrm{BiS}_{2}$ obtained from the Rietveld refinement of the diffraction data. $R_{w p}$ and $\chi^{2}$ correspond to the weighted $R$ factor and chi-square. The $\mathrm{O} / \mathrm{F}$ site is at $(0,0,0) \ldots \ldots 62$

4.2 A list of Bi-S1 bond lengths in the local structure. . . . . . . . . . 78

5.1 Crystal structure parameters of $\mathrm{NdO}_{0.8} \mathrm{~F}_{0.2} \mathrm{BiS}_{2}$ obtained from the Rietveld refinement. . . . . . . . . . . . . . . . . . 87

5.2 Crystal structure parameters of $\mathrm{NdO}_{0.6} \mathrm{~F}_{0.4} \mathrm{BiS}_{2}$ obtained from the Rietveld refinement. ...................... 88

6.1 The tested model of $P 4 / n m m$ symmetry. . . . . . . . . . . . . . . 111

6.2 The tested model of $P 4 m m$ symmetry. . . . . . . . . . . . . . . . . . 111

6.3 The tested model of $P 2{ }_{1} m n$ symmetry. . . . . . . . . . . . . . . . . . 111

6.4 The tested model of $P \overline{4} m 2$ symmetry. . . . . . . . . . . . . . . . . . . . 112

6.5 The tested model of $P 2_{1} / m$ symmetry. . . . . . . . . . . . . . . . 112

6.6 A list of crystal structure parameters for $\mathrm{LaOBiS}_{2}$ obtained from the neutron diffraction data refinement using ShelX. The P1 symmetry was used in the refinement. . . . . . . . . . . . . . . 116 
Dedicated to my parents... 


\section{Chapter 1}

\section{Introduction}

\subsection{Introduction}

Over a century since the discovery of superconductivity by Kamerlingh Onnes at Leiden Laboratory in 1911 [1], scientists have been designing new superconducting structures with the ultimate goal of achieving room temperature superconductivity. Elements, alloys, intermetalic compounds, organics, perovskites, stacked fullerenes and layered compositions $[2-7]$ are among the various structures of superconductors. The $\mathrm{BiS}_{2}$ superconductor class that was discovered in 2012 [8] can be included in the layered superconductor category. Similarly to the high $\mathrm{T}_{c}$ cuprates containing $\mathrm{CuO}$ planes and iron based superconductors containing Fe-chalcogen/pnictogen planes, this recently found superconductor class has $\mathrm{BiS}_{2}$ planes as the common building block as its name implies. The superconducting properties of the $\mathrm{BiS}_{2}$ class can be changed by the type of doping on the donor layer that stack alternatively with the superconducting layer.

There are two fundamental characteristics of any type of superconductor. 1. Superconductors can carry a resistance-free electric charge flow below a critical temperature $\mathrm{T}_{c} \cdot[1]$ 
2. Screening currents that run across the surface of a superconductor generate an exterior magnetic field opposite to the applied magnetic field and repel it which is called the Meissner effect[9]. The inherent question is how does the superconductivity occur. The first definite microscopic explanation to the underlying mechanism of superconductivity was published by Bardeen, Cooper and Schrieffer in 1957, [10-12] which is known as the BCS theory. The BCS theory explains superconductivity in terms of bound electron pairs known as Cooper pairs, forming as a result of the electron-phonon interaction: the first electron moves through the lattice, attracts the positive ions, and the accumulated positive charge attracts the second electron making a Cooper pair as depicted in Fig. 1.1. These electron-electron interactions via lattice deformations may extend to 100 to $1000 \mathrm{~nm}$ apart, therefore weak and tend to break easily due to thermal fluctuations at room temperature. Constituting of two spin 1/2 Fermi particles with opposite momentum and opposite spin, the Cooper pair is a spin zero bosonic state. The ground state energy of this bosonic state is gapped from the energy levels of the unpaired electrons by the energy needed to break up a Cooper pair. In the energy gap, no eigenenergy is allowed. Thus the Cooper pairs condensate moves collectively without friction given no dissipative scattering occurs. The ratio of $2 \Delta / \mathrm{k}_{B} \mathrm{~T}$ ( $\Delta$ - superconducting gap at 0 $\mathrm{K}, \mathrm{k}_{B}$ - Boltzmann constant) or the reduced energy gap resulted by the BCS theory in the weak coupling regime (coupling constant $\lambda \ll 1$ ) where the pairing is confined to a thin shell of energies near the Fermi surface is 3.5.[13] The energy gap $\Delta_{T}$ of a superconductor depends on the temperature $\mathrm{T}$, and in the vicinity of critical temperature the BCS theory yields $\Delta_{T}=1.74 \Delta\left[1-\mathrm{T} / \mathrm{T}_{c}\right][6]$, and the gap, $\Delta_{T}$, exists for temperatures below $\mathrm{T}_{c}$ and becomes zero at the finite temperature $\mathrm{T}=\mathrm{T}_{c}$. The maximum $\mathrm{T}_{c}$ that can be achieved in the weak coupling limit is $\sim 20 \mathrm{~K}$. $[6,14]$ 


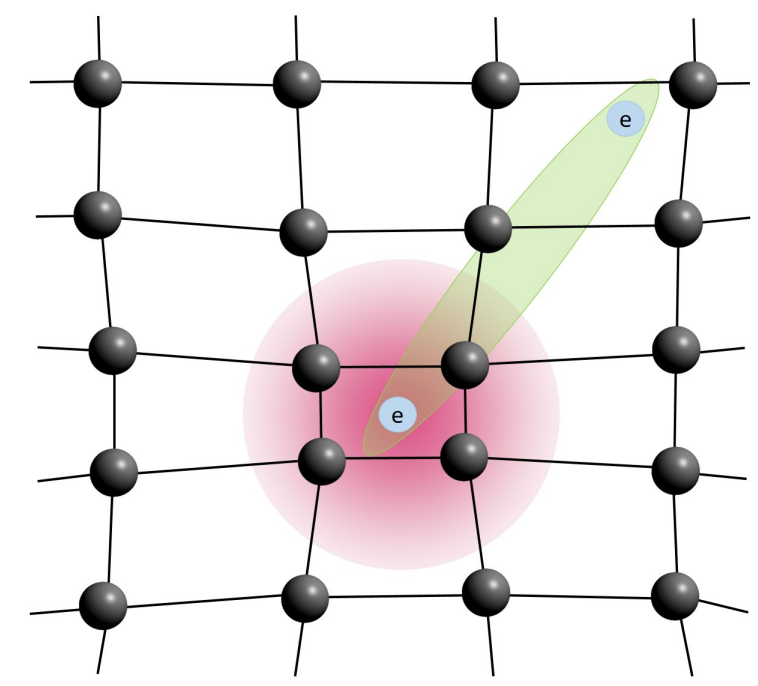

Figure 1.1: The Cooper pair (green oval) formation of electrons (blue circles). The electron is attracted to the positive charge density (pink glow) created by the first electron around itself by distorting the lattice.

The $\mathrm{BiS}_{2}$ systems are phononic superconductors $[15,16]$ with an electron-phonon coupling constant $\sim 0.8$ indicating strong coupling. [17, 18] The experimental results from magnetic penetration depth, muon spin rotation spectroscopy and angle-resolved photoemission spectroscopy (ARPES) also support conventional BCS superconductivity mediated by electron phonon coupling [19-21]. However, scanning tunneling spectroscopy (STS) measurements show two superconducting gaps exist in $\mathrm{NdO}_{1-x} \mathrm{~F}_{x} \mathrm{BiS}_{2}$. The reduced gap value, $2 \Delta / \mathrm{k}_{B} \mathrm{~T}$, for the smaller gap is 16.8 in the vicinity of bulk superconductivity and deviates significantly from the BCS value. $[22,23]$. In addition, the normal state (i.e the state after the superconductivity is suppressed) being non-metalic also contributes to this indefiniteness whether $\mathrm{BiS}_{2}$ superconductors can be categorized as conventional BCS type or not. Furthurmore, theoretical studies using possible spin and orbital fluctuations in combination with electron correlations show different signatures that may lead to identify the pairing symmetry.[24] However, no experimental verifications have been reported up to date. Therefore, it is still not conclusive whether 
the superconducting mechanism of $\mathrm{BiS}_{2}$ class is conventional electron-phonon mediated (BCS) or exotic type as in high $\mathrm{T}_{c}$ superconductors.

There are several families of $\mathrm{BiS}_{2}$ class; $\mathrm{Bi}_{6} \mathrm{O}_{4} \mathrm{~S}_{4}\left(\mathrm{SO}_{4}\right)_{1-x}$ [8], $\mathrm{ReO}_{1-x} \mathrm{~F}_{x} \mathrm{BiS}_{2}(\mathrm{Re}=\mathrm{La}$, Nd, Pr, Ce, and $\mathrm{Yb})[25,26], \mathrm{La}_{1-x} \mathrm{M}_{x} \mathrm{OBiS}_{2}\left(\mathrm{M}=\mathrm{Ti}, \mathrm{Zr}, \mathrm{Hf}\right.$ and Th)[27], $\mathrm{Sr}_{1-x} \mathrm{RE}_{x} \mathrm{FBiS}_{2}$ $(\mathrm{RE}=\mathrm{La}, \mathrm{Ce}, \mathrm{Nd}, \mathrm{Pr}$ and $\mathrm{Sm})[28,29]$ and Eu based $\mathrm{BiS}_{2}$ compounds[30-32]. Superconductivity in $\mathrm{Bi}_{6} \mathrm{O}_{4} \mathrm{~S}_{4}\left(\mathrm{SO}_{4}\right)_{1-x}$ and $\mathrm{EuFBiS}_{2}$ is induced by self-doping into the $\mathrm{BiS}_{2}$ layers via the introduction of defects in the $\mathrm{SO}_{4}$ layer and the mixed valence of $\mathrm{Eu}^{2.2+}$ [30], respectively, which produce electron carriers into the $\mathrm{BiS}_{2}$ superconducting layers. In $\mathrm{La}_{1-x} \mathrm{M}_{x} \mathrm{OBiS}_{2}$ and $\mathrm{Sr}_{1-x} \mathrm{RE}_{x} \mathrm{FBiS}_{2}$, superconductivity emerges with electron doping via rare earth element doping, while in $\mathrm{ReO}_{1-x} \mathrm{~F}_{x} \mathrm{BiS}_{2}$, the focus of this study, superconductivity is induced by electron doping via fluorine substitution at the oxygen sites. $\mathrm{LaO}_{1-x} \mathrm{~F}_{x} \mathrm{BiS}_{2}$ shows a peculiar relation of its crystal structure to superconductivity. The parent compound shows high crystallinity, and upon doping, superconductivity is maximized when crystal disorder is induced by high pressure annealing.

The $\mathrm{BiS}_{2}$ class shows that disorder and defects play a key role in superconductivity. The disorder can be manifested by atomic modes that break site symmetries. It was theoretically shown that $\mathrm{BiS}_{2}$ superconductors involve rich physics with atomic modes that foster a charge density wave (CDW) instability upon electron doping and spin-orbit (SO) interactions with spin polarization. [15, 33] A brief background of (a) disorder (b) CDW and SO coupling, and (c) structure-property relationship in superconductivity will be presented in the following paragraphs.

The central role of disorder in $\mathrm{BiS}_{2}$ superconductivity is reminiscent of amorphous superconductors. The destruction of long range order in transforming metals to their amorphous phase by a process such as quenched condensation (Evaporating the metal in high 
vacuum and condensing the vapor onto a substrate at He-temperature), ion implantation or liquid quenching, causes clear differences in the superconducting properties.[34, 35] In most of the cases, the amorphous disorder is found to enhance superconductivity in the simple metals. For example, the quench condensed amorphous Bi and Ga show a $\mathrm{T}_{c} \sim 6.1$ $\mathrm{K}$ and $8.4 \mathrm{~K}$, respectively, while their crystalline phases are very poor superconductors $[34,36]$. Amorphous disorder leads to weakening of atomic bond lengths and promotes softening of the average phonon spectrum. This corresponds to a reduction in phonon frequency and thereby increases the electron-phonon interaction.[37]

The CDW instability recollects the important role it plays in the high $\mathrm{T}_{c}$ cuprate superconductivity. CDWs had been observed directly as periodic incommensurate structures in superconducting $\mathrm{Bi}_{2} \mathrm{Sr}_{2} \mathrm{CaCu}_{2} \mathrm{O}_{8+\delta}(\mathrm{BSCCO})[38]$ and as charge-ordered checkerboard state in $\mathrm{Ca}_{2-x} \mathrm{Na}_{x} \mathrm{CuO}_{2} \mathrm{Cl}_{2}$ [39]. Scanning tunnel microscopy (STM) measurements show CDWs in $\mathrm{Bi}_{2} \mathrm{Sr}_{1.4} \mathrm{La}_{0.6} \mathrm{CuO}_{6+\delta}[40]$ with an incommensurate period and CDW wave vectors Q depend on oxygen doping level. Similarly, nondispersive (energy-independent) checkerboard CDWs have been observed in $\mathrm{Bi}_{2-y} \mathrm{~Pb}_{y} \mathrm{Sr}_{2-z} \mathrm{La}_{z} \mathrm{CuO}_{6+x}$ [41] with $\mathrm{Q}$ substantially depending on doping. Also, the CDW diffraction patterns are found in both directions of $\mathrm{CuO}$ bonds in the $\mathrm{CuO}_{2}$ plane, and the $\mathrm{CDW}$ correlation lengths parallel and perpendicular to the planes in $\mathrm{YBa}_{2} \mathrm{Cu}_{3} \mathrm{O}_{x}$ (YBCO)[42] where the properties of these quasi-2D CDW are found to be strongly affected by disorder. Furthermore, the STM spectra of $\left(\mathrm{Bi}_{1-y} \mathrm{~Pb}_{y}\right)_{2} \mathrm{Sr}_{2} \mathrm{CuO}_{6+x}$ are identified to be contributed by two gaps, where the homogeneous one is the superconducting gap and the larger, inhomogeneous gap is equivalent to a CDW gap.[43] Also the specific heat measurements on 
$\mathrm{Bi}_{2} \mathrm{Sr}_{2-x} \mathrm{La}_{x} \mathrm{CuO}_{6+\delta}$ demonstrated the discontinuity of specific heat at the superconducting transition may also serve as indirect evidence for a CDW gap, as the discontinuity is much below the corresponding BCS values.[44, 45] Spin orbit (SO) coupling on the other hand, plays a significant role in superconductivity of the oxide interfaces such as $\mathrm{SrTiO}_{3} / \mathrm{LaAlO}_{3}$. [46] It was shown that the spin orbit coupling arising as a result of inversion symmetry breaking can be tuned to establish superconductivity between these oxide interfaces.

In explaining superconducting mechanism, crystal structure plays a crucial role since superconductivity is a collective phenomenon emerging as a result of different possible coupling between lattice, electrons, orbitals and spins. [6, 47-49] Therefore, superconductivity can be changed either by internal or external parameters such as charge doping, isovalent substitutions and pressure. For example, consider LnFeAsO ( $\mathrm{Ln}=$ lanthanide), of which the superconductivity is induced by fluorine doping at oxygen site. Replacing the Ln site from La to Sm increases $\mathrm{T}_{c}$ from $26 \mathrm{~K}$ to $55 \mathrm{~K}$ [50] whereas replacing the $\mathrm{Ln}$ site with As to $\mathrm{P}$ in LaFeAsO decreases the $\mathrm{T}_{c}$ to $4 \mathrm{~K}$. [51] With an external pressure of $4 \mathrm{GPa}$, it was shown that the $\mathrm{T}_{c}$ onset of fluorine doped LnFeAsO increases to $43 \mathrm{~K}$.

The anion height of LnFeAsO can be changed by different doping and deficiency levels at $\mathrm{Ln}$ site and oxygen site, respectively. With oxygen deficiency level of $\mathrm{LnFeAsO}_{1-y}$ superconductor, the anion height increases more rapidly in the $\mathrm{Ln}=\mathrm{Nd}$ compounds than in the Ln=La compounds. [53] This change of anion height is shown to be a good candidate in tuning the $\mathrm{T}_{c}$ of iron based superconductors. As shown in Fig. 1.2, the $\mathrm{T}_{c}$ can be optimized by tuning the anion height and it is applicable to most of the Febased superconductors. [54] Coupled to this are the As-Fe-As bond angles which with 
increasing oxygen deficiency, approach a regular $\mathrm{FeAs}_{4}$ tetrahedron in which $\alpha$ and $\beta$ are $109.47^{\circ}$ ( $\alpha$ and $\beta$ are marked in Fig. 1.3). Also upon cooling, it was shown that the FeAs $4_{4}$-tetrahedra become more regular. The relationship between $\mathrm{T}_{c}$ and $\mathrm{FeAs}_{4}{ }^{-}$ tetrahedral distortion is shown in Fig. 1.3. The tetrahedral distortion is represented by the As-Fe-As bond angle. The $\mathrm{T}_{c}$ increases as the tetrahedral distortion decreases and maximizes when the tetrahedron becomes regular. [53]

What is the role of structure in the superconducting mechanism of $\mathrm{BiS}_{2}$ class? In an attempt to address this fundamentally important question, the structure of $\mathrm{LnO}_{1-x} \mathrm{~F}_{x} \mathrm{BiS}_{2}$ $(\mathrm{Ln}=\mathrm{La}, \mathrm{Nd}$ and $\mathrm{Pr})$ was investigated employing neutron and synchrotron $\mathrm{x}$-ray scattering on polycrystalline and single crystal samples. Dopants cause the lattice to rearrange, be it in a large scale as in crystal structure or in a small scale as in local structure. The structural investigations were carried out at different fluorine doping levels as a function of temperature to investigate the lattice rearrangements and their implications on $\mathrm{BiS}_{2}$ superconductivity.

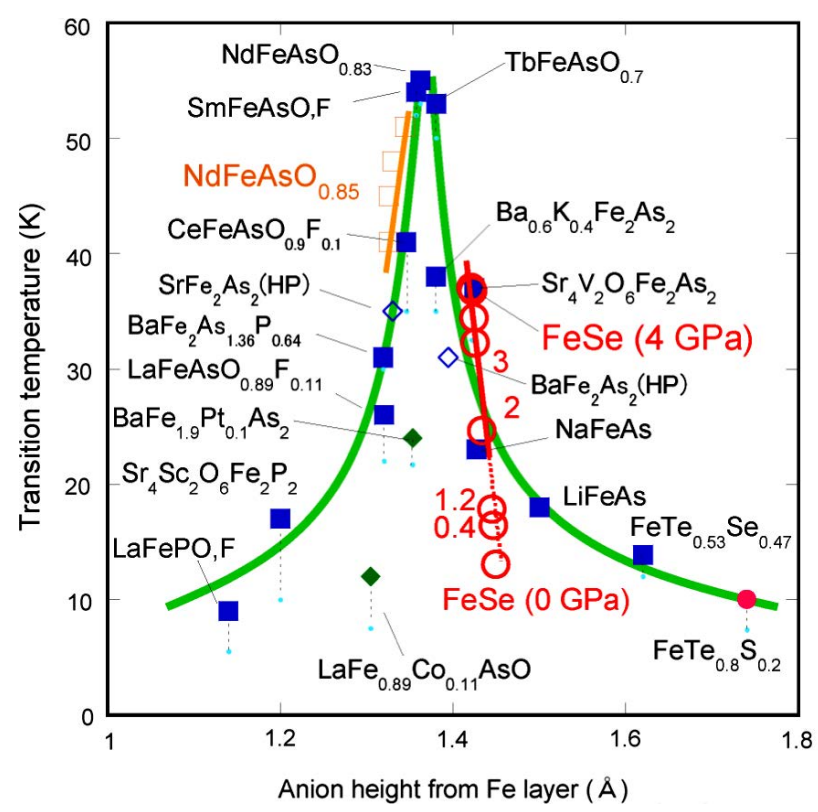

Figure 1.2: The anion height dependence of $\mathrm{T}_{c}$ in Fe-based superconductors. The curve peaks at $\sim 1.38 \AA[54]$ 

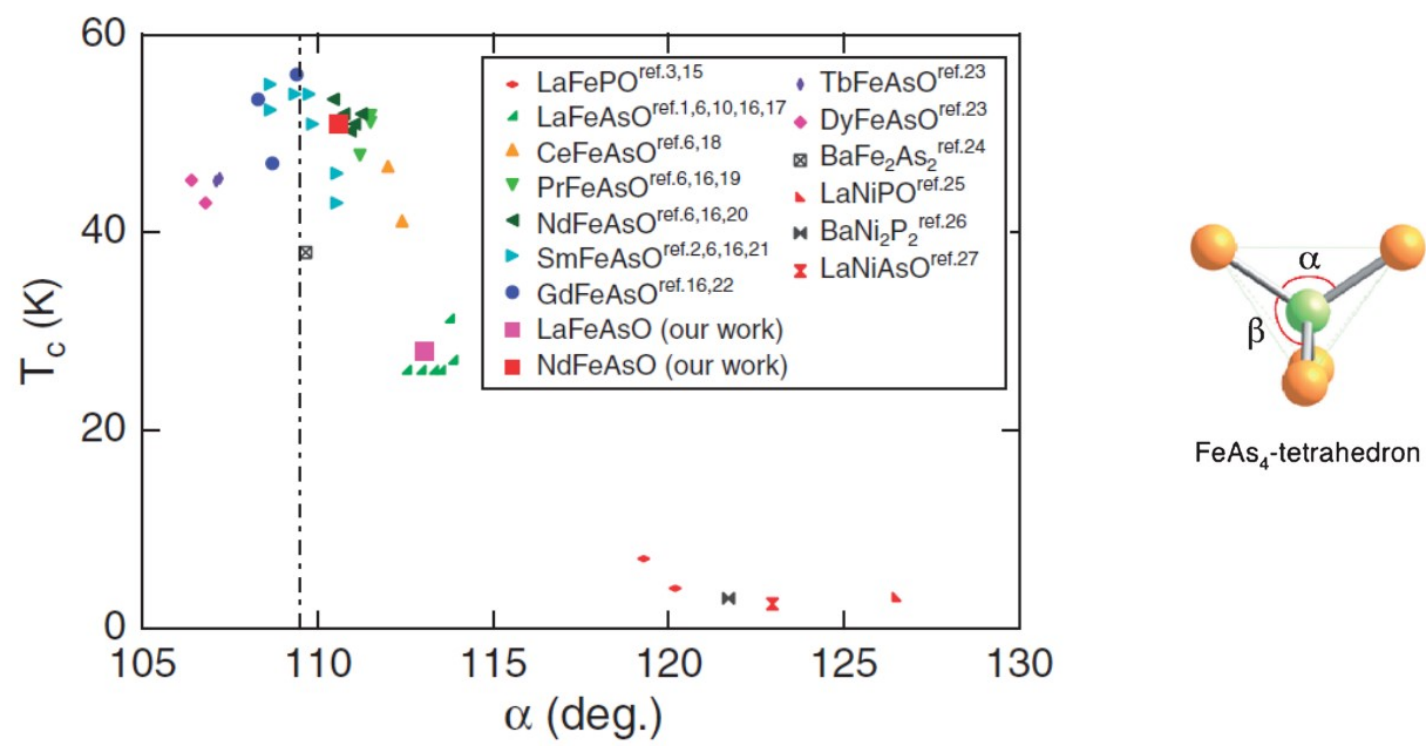

Figure 1.3: The $T_{c}$ vs AsFeAs bond angle for various pnictide superconductors. The AsFeAs bond angle determines the distortion of the Fe tetrahedron. Formulas of parent compositions of superconductors are shown in the inset. Crystal structure parameters of the samples are selected at the maximum $\mathrm{T}_{c}$ of each system. The vertical dashed line indicates the bond angle of a regular tetrahedron.[53]

\subsection{Layout of the Dissertation}

The next chapters of the dissertation are organized as follows. In chapter 2 , the background work that have been carried out on $\mathrm{BiS}_{2}$ superconductor class is provided. The summarized results serve as the foundation to discuss the outcomes in the following chapters. In chapter 3 , an overview to the theoretical background based for the neutron and synchrotron x-ray diffraction experiments is presented and the experimental methods used for data collection, data analysis and sample preparation are described. In chapter 4, the crystal structure of $\mathrm{LaO}_{1-x} \mathrm{~F}_{x} \mathrm{BiS}_{2}(x=0,0.2,0.3$ and 0.5$)$ is presented using neutron diffraction experiments on polycrystalline samples. The possible atomic distortions that explain the difference between the average crystal structure and local structure as a function of doping and temperature are described. The Debye temperature of the parent sample and the superconducting $x=0.5$ sample are also presented. In 
chapter 5 , the differences of crystal structure and nature of local lattice distortions upon replacing La with isovalent $\mathrm{Nd}$ are presented using neutron diffraction experiments on polycrystalline samples. The structural differences are described for two doping levels of fluorine, non-superconducting $x=0.2$ and superconducting $x=0.4$, as a function of temperature. In Chapter 6, the crystal structure of $\mathrm{LnO}_{1-x} \mathrm{~F}_{x} \mathrm{BiS}_{2}(\mathrm{Ln}=\mathrm{La}, \mathrm{Nd}$ and Pr) is presented using neutron and synchrotron x-ray experiments on single crystals. Ferro-distortive type atomic distortions are proved to exist in all the samples that can account for supperlattice Bragg peak patterns. In chapter 7, the results are summarized and the final conclusions are presented. 


\section{Chapter 2}

\section{Background}

\subsection{Introduction to $\mathrm{BiS}_{2}$ Superconductors}

$\mathrm{BiS}_{2}$ superconductors opened a new research field in designing novel superconductors and innovative functional materials. $[26,55,56]$ The first superconductor of the $\mathrm{BiS}_{2}$ class is $\mathrm{Bi}_{4} \mathrm{O}_{4} \mathrm{~S}_{3}$ which was later identified as the derivation of the nominal composition of $\mathrm{Bi}_{6} \mathrm{O}_{4} \mathrm{~S}_{4}\left(\mathrm{SO}_{4}\right)_{1-x}$ with $50 \%$ deficiency of $\mathrm{SO}_{4}$ ions. The phase without defects, $x=0$ (see Fig. 2.1(a)), is a band insulator. [8, 57]. The $\mathrm{SO}_{4}$ defficiency introduces charge carriers into the $\mathrm{BiS}_{2}$ superconducting layers. However, the precise control of doping via defects has been reported to be extremely difficult in this system.[55] The magnetic susceptibility, resistivity and specific heat measurements of $\mathrm{Bi}_{4} \mathrm{O}_{4} \mathrm{~S}_{3}$ are shown in Fig. 2.2. The large diamagnetic signal in Fig. 2.2(a) indicates the bulk superconducting states below $\mathrm{T}_{c}$. Fig. 2.2(b) shows the temperature dependence of electrical resistivity with the onset of $\mathrm{T}_{c}$ at $\sim 5 \mathrm{~K}$ and the zero-resistivity state below $4.5 \mathrm{~K}$. As shown in Fig. 2.2(c), the specific heat jump associated with superconducting transition also shows consistent results for bulk superconductivity occurring at $\sim 4.5 \mathrm{~K}$. 


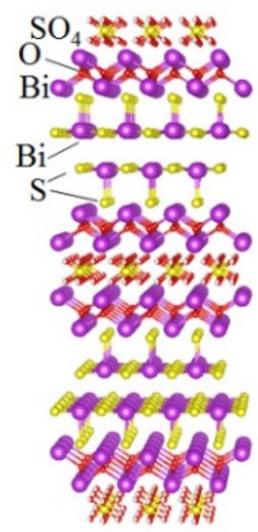

(a) $\mathrm{Bi}_{6} \mathrm{O}_{4} \mathrm{~S}_{4}\left(\mathrm{SO}_{4}\right)$

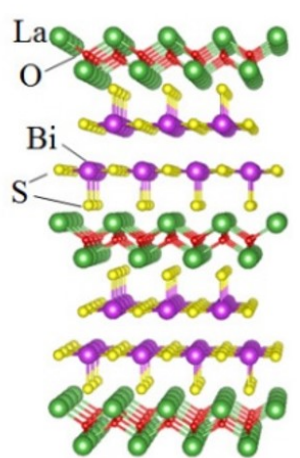

(b) $\mathrm{LaOBiS}_{2}$

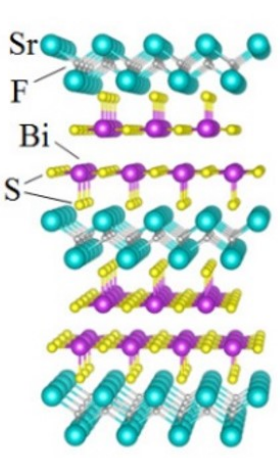

(c) $\mathrm{SrFBiS}_{2}$

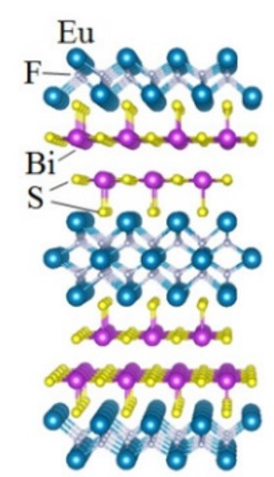

(d) $\mathrm{Eu}_{3} \mathrm{~F}_{4} \mathrm{Bi}_{2} \mathrm{~S}_{4}$

Figure 2.1: Schematic diagrams of layered crystal structures of $\mathrm{BiS}_{2}$ based superconductors, (a) $\mathrm{LaOBiS}_{2}$ (b) $\mathrm{SrFBiS}_{2}$ (c) $\mathrm{Eu}_{3} \mathrm{~F}_{4} \mathrm{Bi}_{2} \mathrm{~S}_{4}$ and (d) $\mathrm{Bi}_{6} \mathrm{O}_{4} \mathrm{~S}_{4}\left(\mathrm{SO}_{4}\right)$. [55]

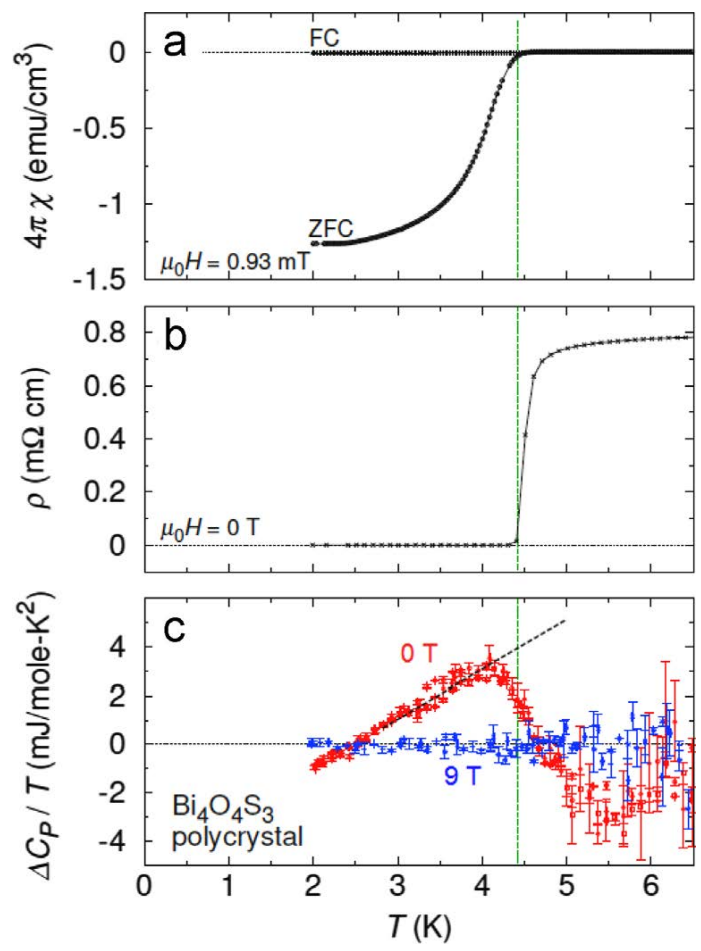

Figure 2.2: (a) magnetic susceptibility, (b) electrical resistivity and (c) specific heat measurements for the superconductor $\mathrm{Bi}_{4} \mathrm{O}_{4} \mathrm{~S}_{3}$ as a function of temperature. [58][59]

$\mathrm{BiS}_{2}$ superconductors have the characteristic structure of an alternating stacking of superconducting $\mathrm{BiS}_{2}$ bi-layers and blocking layers. As shown in Fig. 2.1 (b), (c) and (d), in LaOBiS2, $\mathrm{La}_{2} \mathrm{O}_{2}$ blocking layer can be replaced with $\mathrm{Sr}_{2} \mathrm{~F}_{2}$ and $\mathrm{Eu}_{2} \mathrm{~F}_{2}$ to 
make $\mathrm{SrFBiS}_{2}$ and $\mathrm{Eu}_{3} \mathrm{~F}_{4} \mathrm{Bi}_{2} \mathrm{~S}_{4}$. LaOBiS2 and $\mathrm{SrFBiS}_{2}$ have close lattice constants that make their physical properties quite similar thus giving the option of carrier doping and pressure techniques applied in a similar way. [55] The $\mathrm{ReO}_{1-x} \mathrm{~F}_{x} \mathrm{BiS}_{2}$ (La, Nd, $\mathrm{Pr}$ and $\mathrm{Ce}$ ) is composed of $\mathrm{Re}_{2} \mathrm{O}_{2}$ blocking layers and superconductivity is induced by introducing charge carriers in $\mathrm{BiS}_{2}$ layer by doping oxygen $\left(\mathrm{O}^{2-}\right)$ site with fluorine $\left(\mathrm{F}^{-}\right) .[25,26,60,61]$ The parent compositions, $\mathrm{ReOBiS}_{2}$, are either band insulators or bad metals. $[62,63] \mathrm{Eu}_{3} \mathrm{~F}_{4} \mathrm{Bi}_{2} \mathrm{~S}_{4}$ does not need extrinsic doping for superconductivity as the mixed valence of Eu ions dopes the system with charge carriers.[30-32]

\subsection{High pressure-induced superconductivity in $\mathrm{BiS}_{2}$ su- perconductors}

Most of the $\mathrm{BiS}_{2}$ based superconductors including $\mathrm{LnO}_{0.5} \mathrm{~F}_{0} .5 \mathrm{BiS}_{2}$ ( $\mathrm{Ln}=\mathrm{La}, \mathrm{Nd}, \mathrm{Pr}$ and Ce) and $\mathrm{EuFBiS}_{2}$ show enhanced superconductivity with high pressure annealing during the synthesis.[55][31] An exception is $\mathrm{Bi}_{4} \mathrm{O}_{4} \mathrm{~S}_{3}$ which shows a suppressed superconductivity upon high pressure effect.[57] The Fig. 2.3 shows the temperature dependence of magnetic susceptibility of $\mathrm{LaO}_{0.5} \mathrm{~F}_{0} .5 \mathrm{BiS}_{2}$ annealed under ambient conditions and high pressure conditions using the cubic-anvil synthesis instrument at $2 \mathrm{GPa}$. Without high pressure annealing, $\mathrm{T}_{c}$ is $2.7 \mathrm{~K}$ as shown in Fig. 2.3(a) which increases to $10.6 \mathrm{~K}$ with high pressure annealing as shown in Fig. 2.3(b) where the larger diamagnetic signal also shows the increase of shielding volume fraction. Furthermore, it was shown that when the high pressure annealed samples were subsequently annealed under ambient pressure, they returned to the initial state with the lower $\mathrm{T}_{c}$. [64] 

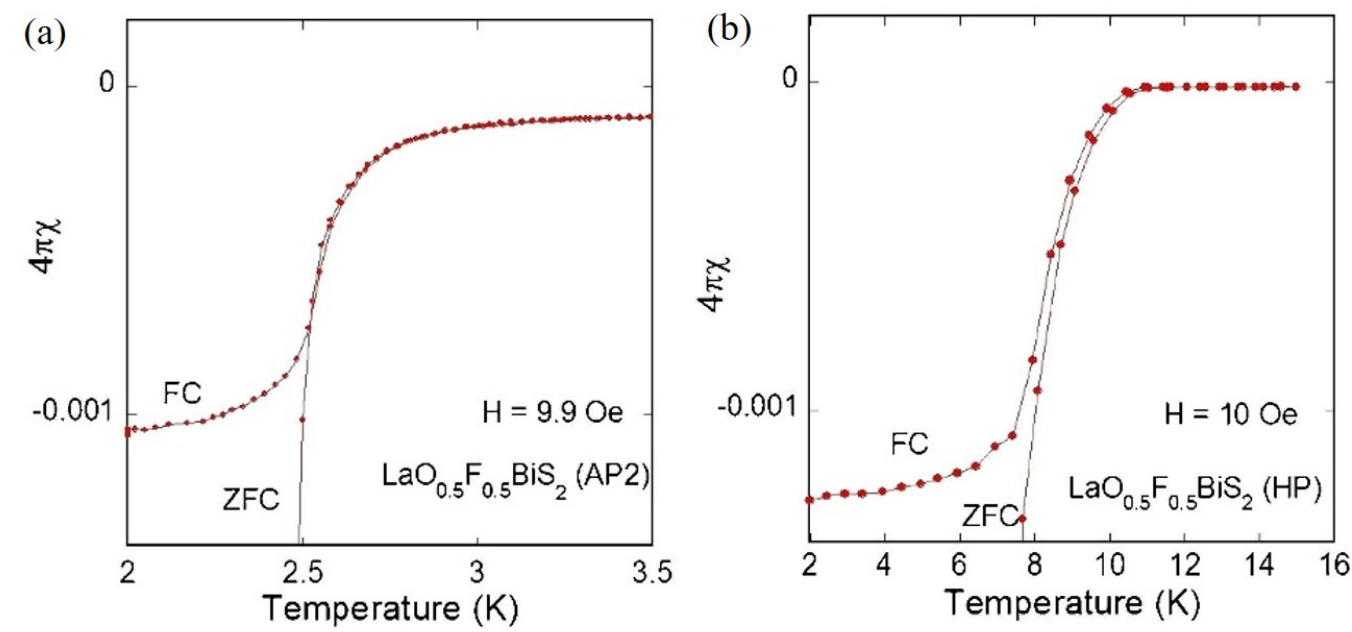

FIgure 2.3: The magnetic susceptibility of $\mathrm{LaO}_{0.5} \mathrm{~F}_{0} .5 \mathrm{BiS}_{2}$ as a function of temperature prepared under (a) ambient conditions (AP2) and (b) high pressure, HP. The high pressured samples are prepared using AP2 process and annealed secondarily with $2 \mathrm{GPa}$ at $600^{\circ} \mathrm{C}$. [25]

The pressure effect on $\mathrm{T}_{c}$ that was observed in high pressure annealed samples has also been observed similarly when the samples were measured under applied pressure. The electrical resistivity measurements of $\mathrm{BiS}_{2}$ superconductors under pressure show that the superconducting critical temperatures initially increase, reach a maximum value and then gradually decrease with increasing pressure. [65-68] In comparison, in high pressure annealed samples, $\mathrm{T}_{c}$ rises very slightly with pressure.[55] As shown in Fig. 2.4, the $\mathrm{PrO}_{0.5} \mathrm{~F}_{0.5} \mathrm{BiS}_{2}$ and $\mathrm{NdO}_{0.5} \mathrm{~F}_{0.5} \mathrm{BiS}_{2}$ show a rapid increase in $\mathrm{T}_{c}$ within a narrow range of pressure $\left(\mathrm{dT}_{c} / \mathrm{d} P \sim 10 \mathrm{~K} \mathrm{GPa}^{-1}\right)$ whereas in $\mathrm{NdO}_{0.5} \mathrm{~F}_{0.5} \mathrm{BiS}_{2}$ and $\mathrm{CeO}_{0.5} \mathrm{~F}_{0.5} \mathrm{BiS}_{2}$ the increase is gradual. All compounds exhibit a semiconducting behavior at low pressure, that has been indicated by a negative temperature coefficient of resistivity $(\mathrm{d} \rho / \mathrm{dT}<0)$. It was found that the $\rho(\mathrm{T})$ can be related to the energy gap, $\Delta$, assuming that the semiconducting behavior originates in the small gap in the band structure. The relationship is described over distinct temperature regions by $\rho(\mathrm{T}) \propto \exp \left(\Delta / \mathrm{K}_{B} \mathrm{~T}\right) .[65,69] \mathrm{In}$ the normal state (the portion above the $\mathrm{T}_{c}(\mathrm{P})$ curves), the semiconducting behavior in 
all compounds is continuously suppressed with increasing pressure that has been manifested by the decrease of the energy gap, $\Delta$, with pressure. The values of the energy gap are indicated in the false colour legend of Fig. 2.4. The $\mathrm{NdO}_{0.5} \mathrm{~F}_{0.5} \mathrm{BiS}_{2}$ sample exhibits a fully metallic state indicated by a positive temperature coefficient of resistivity $(\mathrm{d} \rho / \mathrm{dT}>0)$ at $\sim 2 \mathrm{GPa}$ (where $\Delta$ vanishes), represented by the green region to the right of the crosshatching in Fig. 2.4(b). The magnitude of the transition pressure corresponding to the value of $\mathrm{T}_{c}$ at the mid point of low and high $\mathrm{T}_{c}$ phases is linearly proportional to the atomic number of the rare earth element, Re, as listed in Table 1. Also the pressure-induced increase of $\mathrm{T}_{c}$ decreases with the increasing atomic number. Thus, La has the lowest transition pressure value with the highest increase of $\mathrm{T}_{c}$ out of the Re elements considered.
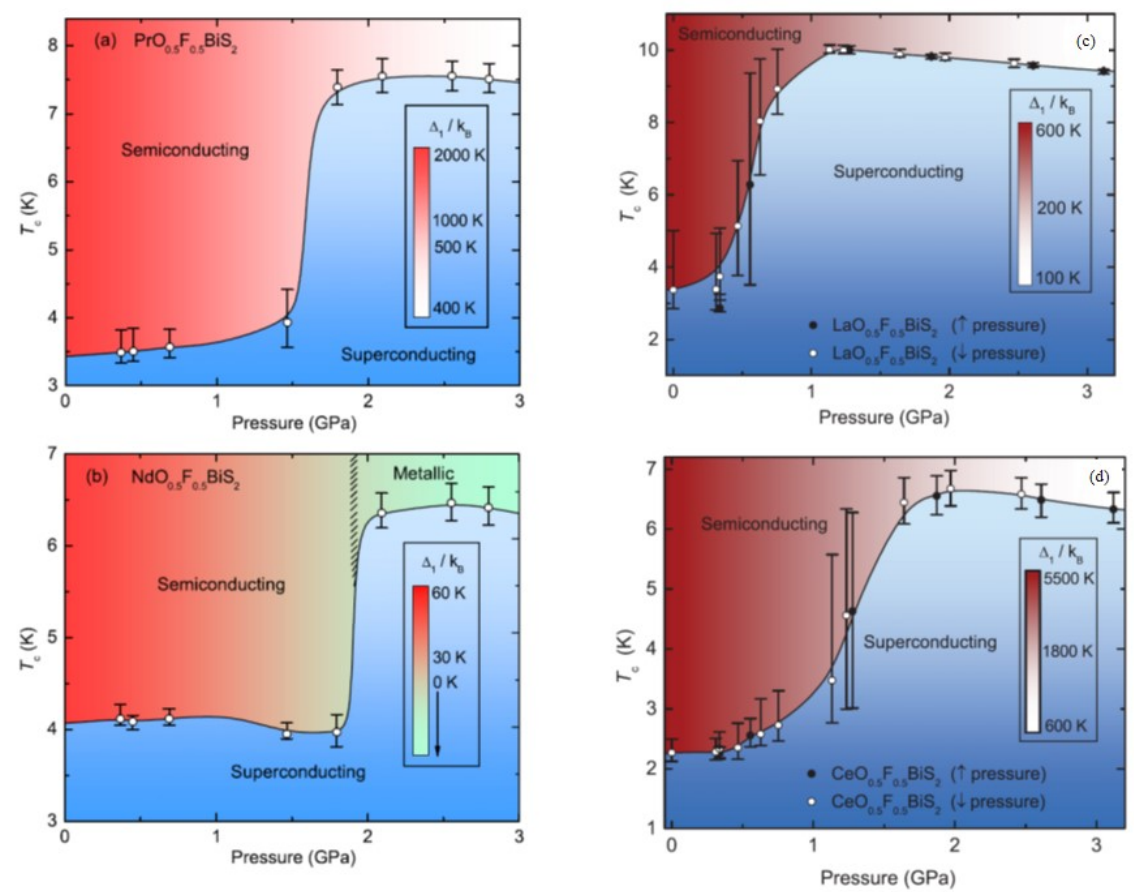

Figure 2.4: Phase diagrams of (a) $\mathrm{PrO}_{0.5} \mathrm{~F}_{0} .5 \mathrm{BiS}_{2}$ (b) $\mathrm{NdO}_{0.5} \mathrm{~F}_{0} .5 \mathrm{BiS}_{2}$ (c) $\mathrm{LaO}_{0.5} \mathrm{~F}_{0} .5 \mathrm{BiS}_{2}$ (d) $\mathrm{CeO}_{0.5} \mathrm{~F}_{0} .5 \mathrm{BiS}_{2}$ under pressure. The length of the vertical bars represent the transition width $\Delta \mathrm{T}_{c}$ and the bar caps represent the $\mathrm{T}_{c}{ }^{\text {onset }}$ (upper) and $\mathrm{T}_{0}$ (lower). The color shows the energy gap $\Delta$ in the semiconducting region where in (b) the green region to the right of the crosshatch corresponds to the metalization of $\mathrm{NdO}_{0.5} \mathrm{~F}_{0} .5 \mathrm{BiS}_{2}(\Delta=0)[65,66]$ 
TABle 2.1: $\mathrm{T}_{c}$ values for $\mathrm{ReO}_{0.5} \mathrm{~F}_{0} .5 \mathrm{BiS}_{2}(\mathrm{Re}=\mathrm{La}, \mathrm{Ce}, \mathrm{Pr}, \mathrm{Nd})$. The $\mathrm{T}_{c}$ and pressure values were obtained from reference [65].

\begin{tabular}{cccccc}
\hline $\mathrm{Re}$ & $\begin{array}{c}\text { Atomic } \\
\text { number }\end{array}$ & Low $\mathrm{T}_{c}(\mathrm{~K})$ & Max $\mathrm{T}_{c}(\mathrm{~K})$ & Increase in $\mathrm{T}_{c}(\mathrm{~K})$ & $\begin{array}{c}\text { Transition } \\
\text { pressure }(\mathrm{GPa})\end{array}$ \\
\hline $\mathrm{La}$ & 57 & 2.9 & 10.1 & 7.2 & 0.56 \\
$\mathrm{Ce}$ & 58 & 1.9 & 6.7 & 4.8 & 1.25 \\
$\mathrm{Pr}$ & 59 & 3.5 & 7.6 & 4.1 & 1.59 \\
$\mathrm{Nd}$ & 60 & 3.9 & 6.4 & 2.5 & 1.91 \\
\hline
\end{tabular}

\subsection{Charge density wave instability in $\mathrm{BiS}_{2}$ superconduc-} tors

The electronic states near the Fermi level of $\mathrm{BiS}_{2}$ superconductors are mainly contributed by Bi $6 p$ and $\mathrm{S} 3 p$ states. $[15,17]$ It was found that the density of states at Fermi level becomes maximum at the half filling $x=0.5$ in $\mathrm{LaO}_{1-x} \mathrm{~F}_{x} \mathrm{BiS}_{2}$ when the $\mathrm{T}_{c}$ reaches a maximum. A strong Fermi surface nesting was shown to occur at $\mathbf{k}=(\pi, \pi, 0)$ which results in a large phonon softening. The nature of the soft phonons depend highly on the doping, with the most negative energy or the most unstable phonon mode at $x=0$ occurring at $\Gamma$ point while at $x=0.5$ the instability occurring along the entire line $(q, q, 0)$ with the most negative energy phonon at $\mathrm{M}$ point as shown in Fig. 2.5.

The most unstable soft phonon at $\Gamma$ point was shown to lower the symmetry of $\mathrm{LaOBiS}_{2}$ from $\mathrm{P} 4 / \mathrm{nmm}$ to $\mathrm{P} 21 \mathrm{mn}$ yielding only a slight lower energy in the system. The distortion involved ferro-electric type movements of sulfur toward the Bi. However, in $x=0.5$, the most unstable phonon lowers the energy of the system significantly and a sinusoidal rearrangement of $\mathrm{S}$ and $\mathrm{Bi}$ atoms gives rise to a charge density wave (CDW) phase as shown in Fig. 2.6. Thus the superconductivity of $\mathrm{LaO}_{1-x} \mathrm{~F}_{x} \mathrm{BiS}_{2}$ superconductors is theoretically shown to occur in the vicinity of lattice instabilities and CDW phases associated with in-plane displacements of sulfur atoms. 

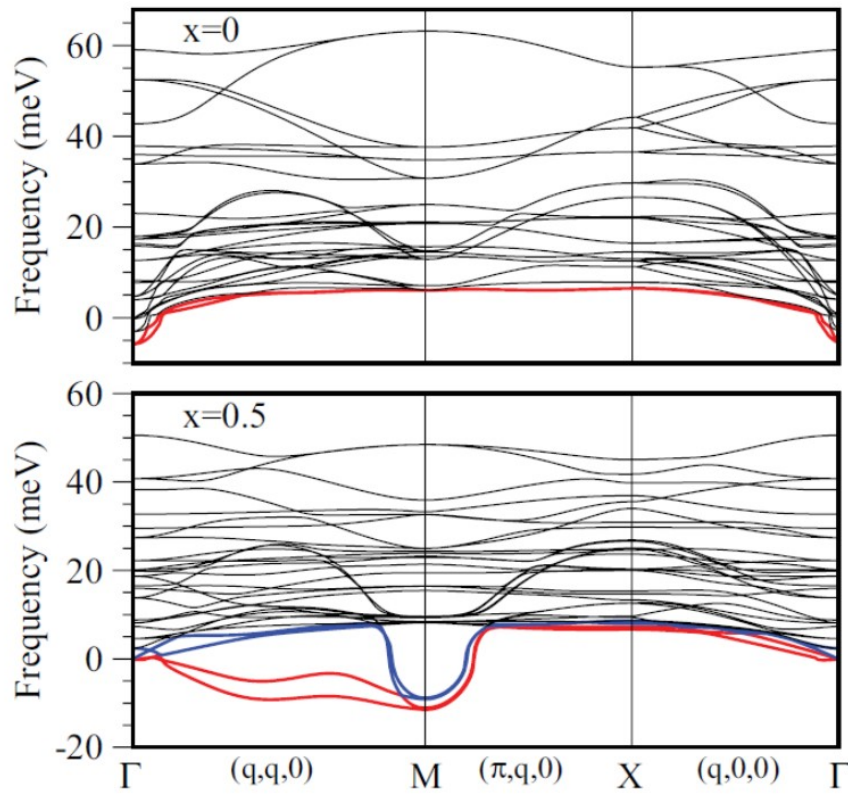

Figure 2.5: Phonon dispersion curves of $\mathrm{LaO}_{1-x} \mathrm{~F}_{x} \mathrm{BiS}_{2}$ at $x=0$ and $x=0.5$. [15]

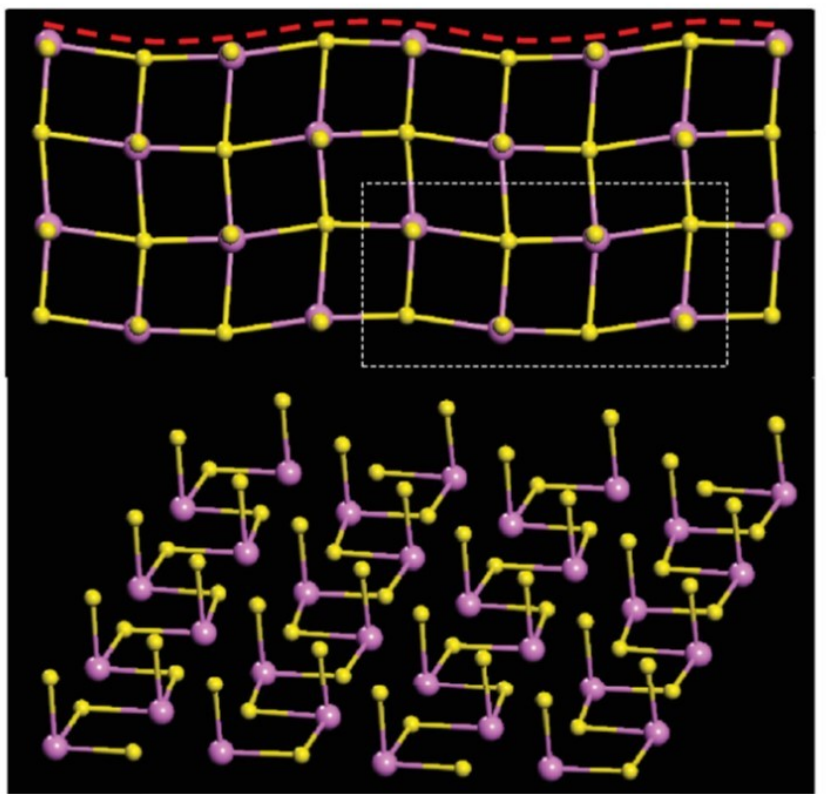

Figure 2.6: The sinusoidal atomic arrangement (top) and the one dimensional channels (bottom) of S and Bi atoms in the CDW phase. The pink and yellow spheres correspond to $\mathrm{Bi}$ and $\mathrm{S}$ atoms, respectively. [15]

\subsection{Hidden spin polarization in $\mathrm{BiS}_{2}$ superconductors}

If the crystalline symmetry of a system is sufficiently low, an effective magnetic field emerges when spin orbit coupling occurs, which consequently leads to spin splitting 
or spin polarization. This phenomenon is known as the Dresselhaus effect in threedimensional (3D) solids arising from bulk inversion asymmetry and Rashba effect in 2D heterostructures arising from 2D structural inversion asymmetry.

$\mathrm{LaOBiS}_{2}$ is a non-magnetic material. Density functional theory calculations showed that Rashba effect is present in $\mathrm{LaOBiS}_{2}$ leading to spin polarization, even though the system is non-magnetic and the crystal lattice is nominally centrosymmetric. It was shown that Rashba effect can occur in even non magnetic materials as $\mathrm{LaOBiS}_{2}$, driven by the lack of inversion symmetry in the site group of an atom rather than the lack of inversion symmetry in the crystal symmetry. However, it was shown that the spin polarization in $\mathrm{LaOBiS}_{2}$ is hidden due to the compensation of their inverted partners in the consecutive $\mathrm{BiS}_{2}$ layers. [33]

\subsection{Theoretically predicted possible symmetry lowering in}

\section{$\mathrm{BiS}_{2}$ superconductors}

It was theoretically predicted that the symmetry of the $\mathrm{BiS}_{2}$ superconductor class could be lower than expected due to a phonon instability observed at $\Gamma$ point and along the entire line $(q, q, 0)$, in $\mathrm{LaOBiS}_{2}$ and $\mathrm{LaO}_{0.5} \mathrm{~F}_{0.5} \mathrm{BiS}_{2}$, respectively, as described in section 2.3. $[15,17]$ The lattice instability drove the symmetry of the parent sample from $P 4 / n m m$ to $P 2{ }_{1} m n$. This involved $\mathrm{S}$ atomic motion toward $\mathrm{Bi}$ atoms along the $a$ axis (or equivalently along $b$ axis) and the distorted phase broke the inversion symmetry. In the $50 \%$ doped compound, the distortion lowered the symmetry to $P 22{ }_{1} 2$. The Bi and S atoms are rearranged giving rise to a sinusoidal distortion. [15] These unstable phonons are shown in Fig. 2.7. 


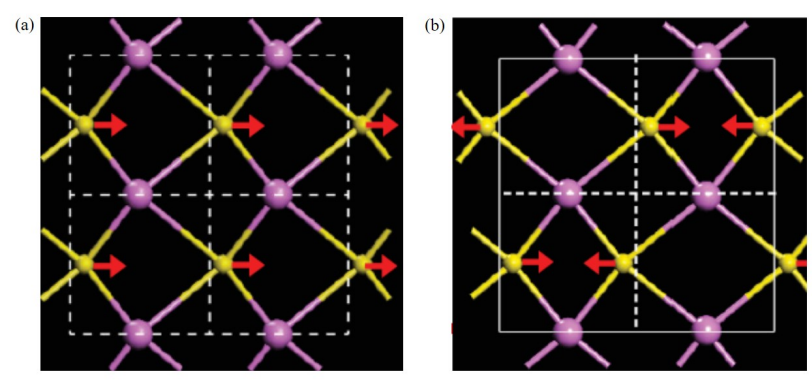

Figure 2.7: The unstable phonons of (a) $\mathrm{LaOBiS}_{2}$ and (b) $\mathrm{LaO}_{0.5} \mathrm{~F}_{0.5} \mathrm{BiS}_{2}$. [15]

Another first principles band structure calculation of $\mathrm{LaO}_{0.5} \mathrm{~F}_{0.5} \mathrm{BiS}_{2}$ had been carried out for tetragonal $P_{4} / n m m$ and monoclinic $P \mathscr{2}_{1} / m$ symmetries. The monoclinic band structure is reported to exhibit a significant splitting at the conduction band bottom of $\mathrm{X}$ point which induces a substantial change of the Fermi surface topology. The origin of the splitting had been shown to arise from strong bilayer coupling induced by symmetry breaking of the crystal structure. [70]

A theoretical study using structural optimization showed that the parent compound has $P 4 / n m m$ symmetry and lowers to $P \overline{4} m 2$ upon $\mathrm{F}$ doping. The atomic bond lengths between $\mathrm{Bi}$ and apical sulfur was shown to expand while the bond between $\mathrm{Bi}$ and in-plane sulfur remained unchanged in this transition between the two symmetries. [18]

Total energy and stability calculations carried out on $\mathrm{LaOBiS}_{2}$ showed the possible coexistence of energetically close polytypes belonging to different symmetries. [71] The proposed symmetries were noncentrosymmetric $\mathrm{P}_{2}{ }_{1} m n$ and $\mathrm{C} 2$, and centrosymmetric $P \mathscr{Q}_{1} / m$ of which the coexistence effectively drives the system into $P 1$ symmetry. As shown in Fig. 2.8, the $\mathrm{P} 4 / \mathrm{nmm}$ exists as an intermediate state in the transition between $P \mathscr{2}_{1} m n$ and $P \mathscr{2}_{1} / m$, with no significant energy difference between the latter two symmetries, thus making it possible for either phases or a mixture of these phases to exist. However, it was shown that when La is substituted with lighter elements or $\mathrm{S}$ is 
substituted with heavier elements, these polytypes do not exist. In this case, the symmetry was shown to be undistorted from its nominal symmetry $P 4 / \mathrm{nmm}$. Furthermore, these calculations showed that the distortions of polytypes can be suppressed by pressure to regain the $P 4 / n m m$ symmetry at a pressure higher than 2.5 GPa. [71] However several experimental studies showed that, with increasing pressure, a phase transition occurs from $\mathrm{P}_{4} / \mathrm{nmm}$ tetragonal structure to $\mathrm{P}_{2} / \mathrm{m}$ monoclinic structure, implying a contrary effect. [72-76]

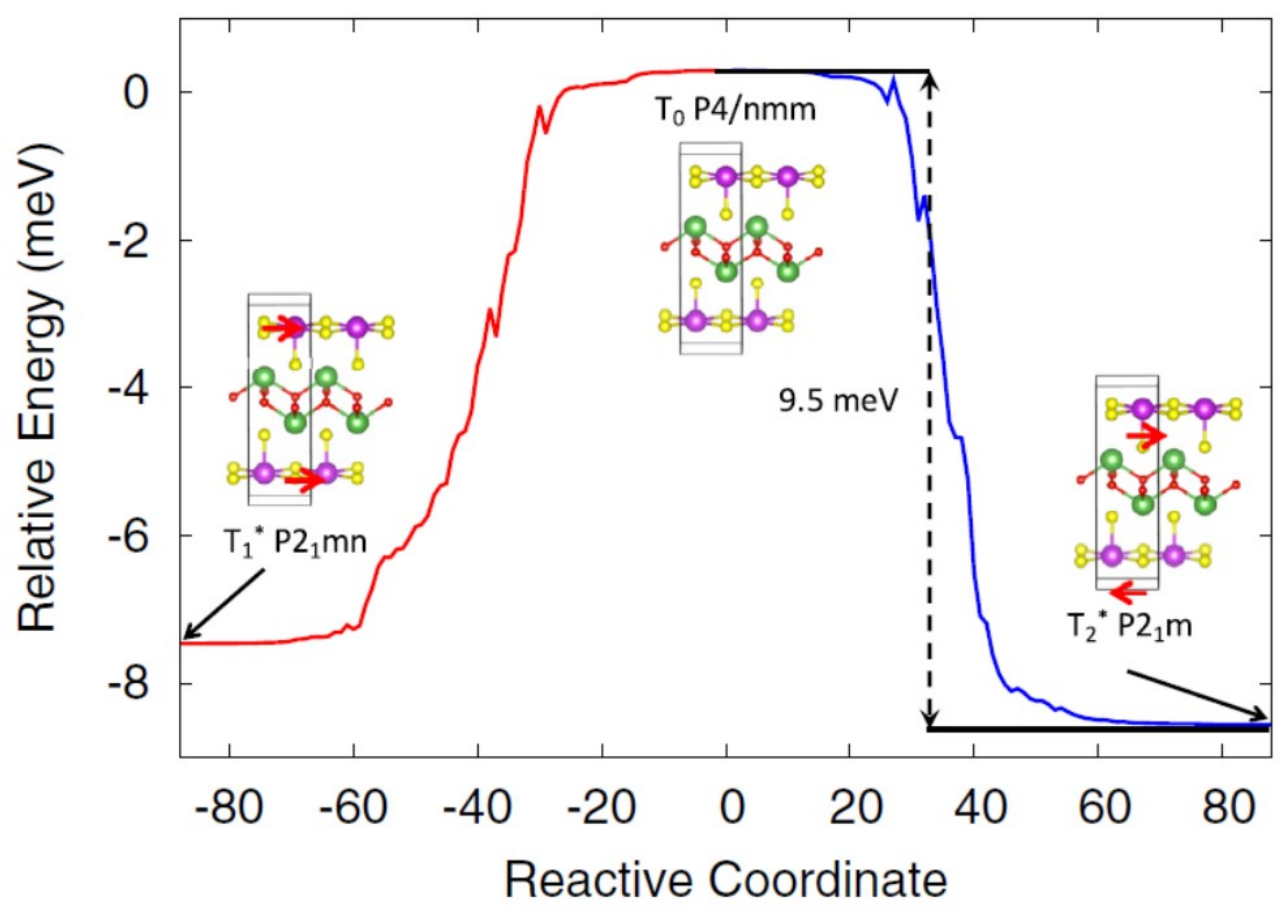

Figure 2.8: Total energy during transformations to final all parameters relaxed polytypes $\mathrm{T}_{1}^{*}$ and $\mathrm{T}_{2}^{*}$. The red arrows show the direction of atomic displacement.[71] 


\subsection{Suggested superconducting mechanisms for $\mathrm{BiS}_{2}$ su-}

\section{perconductors}

A consensus on superconducting mechanisms of $\mathrm{BiS}_{2}$ superconductor class is not reached yet. The diversity in the phenomenology of these materials drove theoretical studies to probe the possibility of conventional as well as unconventional pairing. Among the suggested mechanisms are soft to strong phononic, electronic, spin-orbit coupling mediated and spin-fluctuation mediated pairing.

\subsubsection{Possible conventional pairing}

Electron-phonon coupling calculations had been carried out on $\mathrm{LaO}_{1-x} \mathrm{~F}_{x} \mathrm{BiS}_{2}$ superconductor using frozen phonon method. The large total electron phonon coupling constant, $\lambda \sim 0.8[15,17,18]$ is strong enough to support the experimentally observed $\mathrm{T}_{c}$. This indicated phonon mediated superconductivity in these systems. The estimation of contributions to the large electron phonon coupling indicated 90\% comes from in-plane Bi and S phonons while the remaining $10 \%$ comes from phonons along $c$-axis. The higher energy bands corresponded to pure S oscillations while lower energy bands corresponded to coupled Bi and S motion. Either O/F or La had not shown any significant contribution to electron-phonon coupling.[15] Both theoretical and experimental band structure comparisons confirm the strong electron-phonon coupling in $\mathrm{LaO}_{1-x} \mathrm{~F}_{x} \mathrm{BiS}_{2}$ with or without spin orbit coupling.[17] However, a very recent study on electron-phonon mechanism of this system declared that the electron-phonon coupling strength calculated with an improved density of states method, is much lower than previously calculated value, and 
therefore, conventional phonon-mediated pairing cannot explain the high superconducting transition temperatures observed.[77]

Magnetic penetration depth measurement carried out using the tunnel diode oscillator technique showed that $\mathrm{Bi}_{4} \mathrm{O}_{4} \mathrm{~S}_{3}$ is a conventional s-wave type superconductor with a fully developed gap, hence with strong coupling. They show that the superfluid density which is directly related to magnetic penetration depth, when plotted as a function of $\mathrm{T} / \mathrm{T}_{c}$, follows the single s-wave equation with a gap ratio of 7.18 which is approximately double the value of weak coupling BCS value of 3.5.[19] Muon-spin spectroscopy measurements that can track the evolution of superfluid density with temperature also confirms a fully gapped BCS superconductivity in $\mathrm{LaO}_{0.5} \mathrm{~F}_{0.5} \mathrm{BiS}_{2}$ with a gap ratio of 3.4.[20] High resolution angle-resolved photoemission spectroscopy carried out on $\mathrm{NdO}_{0.5} \mathrm{~F}_{0.5} \mathrm{BiS}_{2}$ shows that two electronlike bands exist around the $\mathrm{X}$ point, forming two small rectangleshaped pockets at the Fermi energy which were found to be much smaller compared to the pockets yielded by band calculations based on the nominal composition. This was attributed to a possible bismuth deficiency. The small Fermi pocket size along with the weak electron-phonon coupling indicated by the energy band renormalization factor, suggested that the superconductivity in this system is conventional BCS type.[21]

\subsubsection{Possible unconventional pairing}

With a majority of studies on $\mathrm{BiS}_{2}$ superconductivity supporting conventional electronphonon mediated mechanism, still there exist indications that the pairing probably be unconventional. In $\mathrm{NdO}_{1-x} \mathrm{~F}_{x} \mathrm{BiS}_{2}$, as shown in Fig. 2.9, the superconductivity is easily destroyed (above $0.6 \mathrm{~T}$ ) when magnetic field $\mathrm{H}$ is applied parallel to $c$ axis while superconductivity is quite strong (retaining even beyond $9 \mathrm{~T}$ ) when $\mathrm{H}$ is applied parallel 
to ab plane which gives rise to a large superconducting anisotropy $\gamma \sim 30-40[23,78]$. $\gamma=\mathrm{H}^{a b}{ }_{c 2} / \mathrm{H}^{c}{ }_{c 2}$ where $\mathrm{H}_{c 2}$ is the upper critical field defined as the value of magnetic field when superconductivity is completely destroyed. An anisotropy of this magnitude is only comparable to that of high $\mathrm{T}_{c}$ Bi-based cuprates such as $\mathrm{Bi}_{2} \mathrm{Sr}_{2} \mathrm{CaCu}_{2} \mathrm{O}_{8+\delta}$ and $\mathrm{Bi}_{2} \mathrm{Sr}_{2} \mathrm{Ca}_{2} \mathrm{Cu}_{3} \mathrm{O}_{10} \cdot[37,79]$ The conventional superconductor, $\mathrm{MgB}_{2}$ only shows a magnetic anisotropy of 1.7-3.[80]
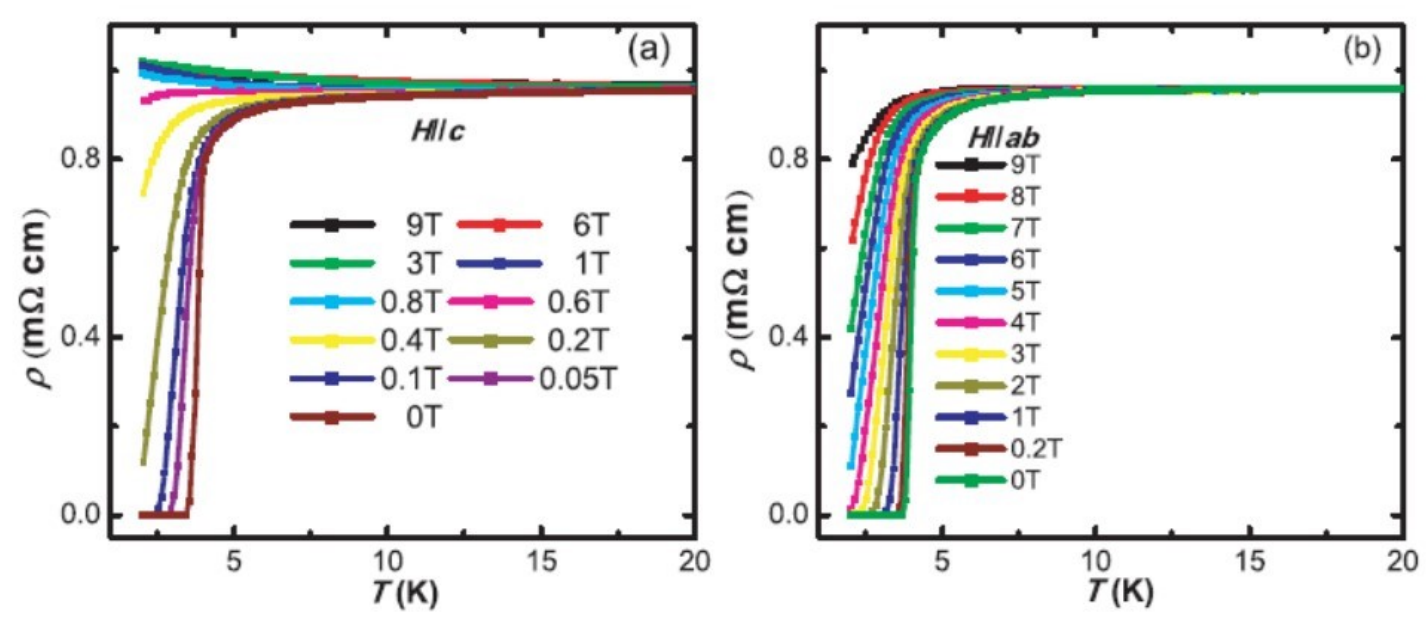

Figure 2.9: The resistivity of $\mathrm{NdO}_{1-x} \mathrm{~F}_{x} \mathrm{BiS}_{2}$ as a function of temperature under different magnetic fields. (a) $\mathrm{H} \| c$ and (b) $\mathrm{H} \| a b$ [23]

In the case of conventional electron phonon mediated pairing, when the transition temperature of the metalic state to the superconducting state is approached, the number of electron pairs formed are fluctuated in a very narrow temperature range by an amount of $\sim 0.1 \mathrm{~T}_{c}$. $[81,82]$ However, in $\mathrm{NdO}_{1-x} \mathrm{~F}_{x} \mathrm{BiS}_{2}$, the superconductivity fluctuation extends up to $20 \mathrm{~K}$ with the bulk superconductivity emerging $\sim 5 \mathrm{~K}$ as shown in Fig. 2.10. This indicates a large deviation from the formation of conventional superconductivity.[23] A muon-spin spectroscopy study showed a double superconducting gap feature in $\mathrm{Bi}_{4} \mathrm{O}_{4} \mathrm{~S}_{3}$ with reduced superconducting gap values of 5.92 and 0.86.[83] Similarly, scanning tunneling spectroscopy showed two gap values in $\mathrm{NdO}_{1-x} \mathrm{~F}_{x} \mathrm{BiS}_{2}$ of which the smaller gap 
corresponds to the bulk superconductivity and its reduced gap ratio is evaluated as 16.8 with the $\mathrm{T}_{c}=4.83$. [23] However, if the reduced gap ratio corresponding to the smaller gap was evaluated at $20 \mathrm{~K}$ up to which the superconductivity fluctuates, it yields a value of 4.06 which does not deviate much from the BCS value. It was suggested that, if this latter pairing at high temperature is correct, it resembles the pseudogap feature shown by some of the cuprate superconductors which may be induced by CDW order[41], or the local paring governed by the valence fluctuation effect of Bi ionic state.[84, 85] However, no evidence has been found for this type of pairing in $\mathrm{BiS}_{2}$ class.

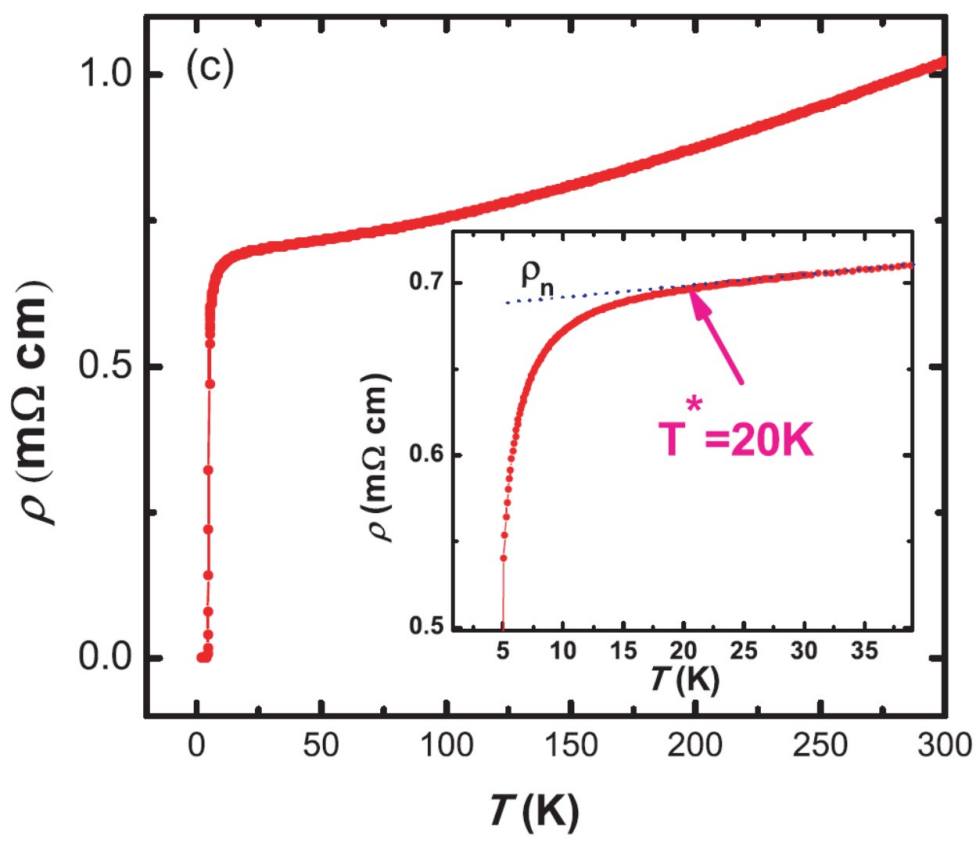

Figure 2.10: The resistivity of $\mathrm{NdO}_{0.5} \mathrm{~F}_{0.5} \mathrm{BiS}_{2}$ as a function of temperature at zero field. The inset shows the superconducting fluctuation up to $20 \mathrm{~K}$ and bulk superconductivity at $5 \mathrm{~K}$. [23]

When the bulk superconductivity is suppressed in $\mathrm{BiS}_{2}$ superconductors, a semiconducting state emerges above $\mathrm{T}_{c}$. [23] However, according to BCS theory, the normal state after the superconductivity is suppressed by an external magnetic field is metalic. For example, the conventional superconductor, $\mathrm{MgB}_{2}$ is a metal in its normal state as shown 
by electron transport measurements.[86] Therefore, considering these results of previous work, the $\mathrm{BiS}_{2}$ class remains more as a new type of conventional superconductors that need further investigation.

\section{7 $\quad$ Suggested possible pairing symmetries}

Several possible pairing states had been suggested while their existence still need to be justified experimentally. Focusing only on the bands that intersect the Fermi level, a two-orbital tight binding model consisting $P_{x}$ and $P_{y}$ orbitals of Bi had been obtained for $\mathrm{LaOBiS}_{2}$. The existence of good Fermi-surface nesting and quasi-one-dimensional nature of bands were shown which gives rise to several possibilities for the superconducting state. The most relevant bands to superconductivity being of $6 p$ character in this model, gives a wide spread of orbitals indicating that the electron-electron interactions may not be strong and the electron-phonon interactions may play the main role. The electronphonon interactions along with the good Fermi surface nesting are proposed to give an enhanced attractive pairing interaction around the nesting vectors giving rise to $s$ wave pairing as shown in Fig. 2.11 (a). However, in the case of short-ranged repulsive interaction, the spin-fluctuation-mediated pairing shows to a sign reversing $s$-wave gap at larger doping and a $d$-wave gap at lower doping as shown in Fig. 2.11 (b) and (c), respectively. Another random phase approximation (RPA) study based on the two orbital model proposed above, links the dominant superconducting gap functions to spin fluctuations, which originate in Fermi surface nesting and are enhanced by electronic interactions. Two symmetries of pairing functions were shown to compete, essentially

with same pairing strength. [24] A similar study showed that the superconducting 
pairing are resulted from strong spin-orbital coupling and the ferromagneticlike spin fluctuations. [87]

(a)

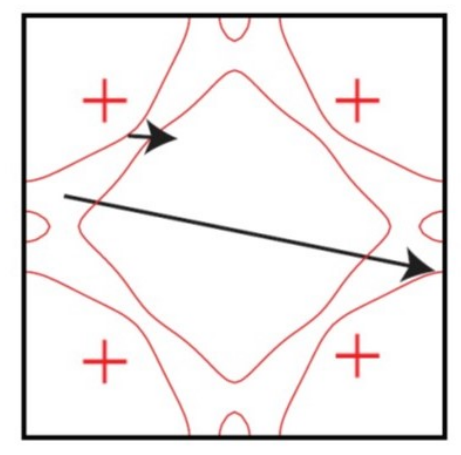

(b)

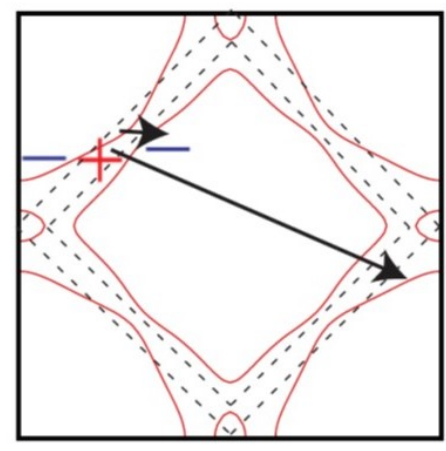

(c)

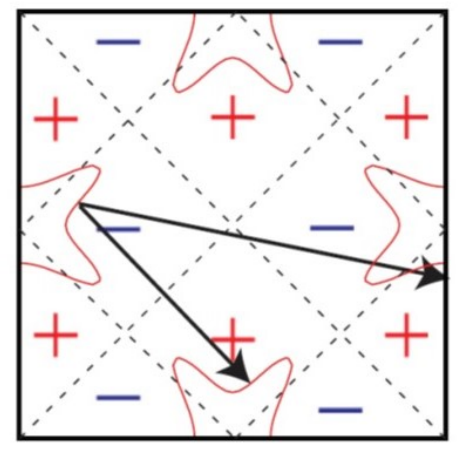

FIgURE 2.11: The Fermi surface of the two orbital model. (a) Sign conserving $s$ wave for attractive pairing interactions such as mediated by phonons or charge fluctuations, (b) sign reversing $s$ wave, and (c) $d$ wave superconducting gaps obtained for repulsive pairing interactions mediated by spin fluctuations. [88]

Different spin signatures are found theoretically by another RPA study for $d$-wave, extended $s$-wave and $p$-wave as shown in Fig. 2.12, which can be used for probing the mechanism of $\mathrm{BiS}_{2}$ superconductors experimentally. [89] For $d$ wave symmetry, as seen from Fig. 2.12(a), the spin fluctuation around $(\pi, \pi)$ is significantly stronger than that of around $(0,0)$ point. For the s-wave pairing symmetry, the spin excitation around $(0$, 0 ) enhances while the spin excitation around $(\pi, \pi)$ is still considerably strong as shown in Fig. 2.12(b). For the triplet pairing symmetry, the transverse spin excitation $\left(\chi^{+-}\right)$ is reported to be stronger than the longitude one $\left(\chi^{z z}\right)$ which make the features of $\chi^{+-}$ experimentally detectable. [89]

Based on the assumptions that electron-electron correlation is important so that the pairing is rather short range and the short range pairing stems from short antiferromagnetic exchange couplings, the extended s-wave pairing symmetry had been shown to favor over d-wave.[90] The extended s-wave pairing is suggested to be tested by measuring the gap distributions at different Fermi surfaces. It was also found that the pairing strength 

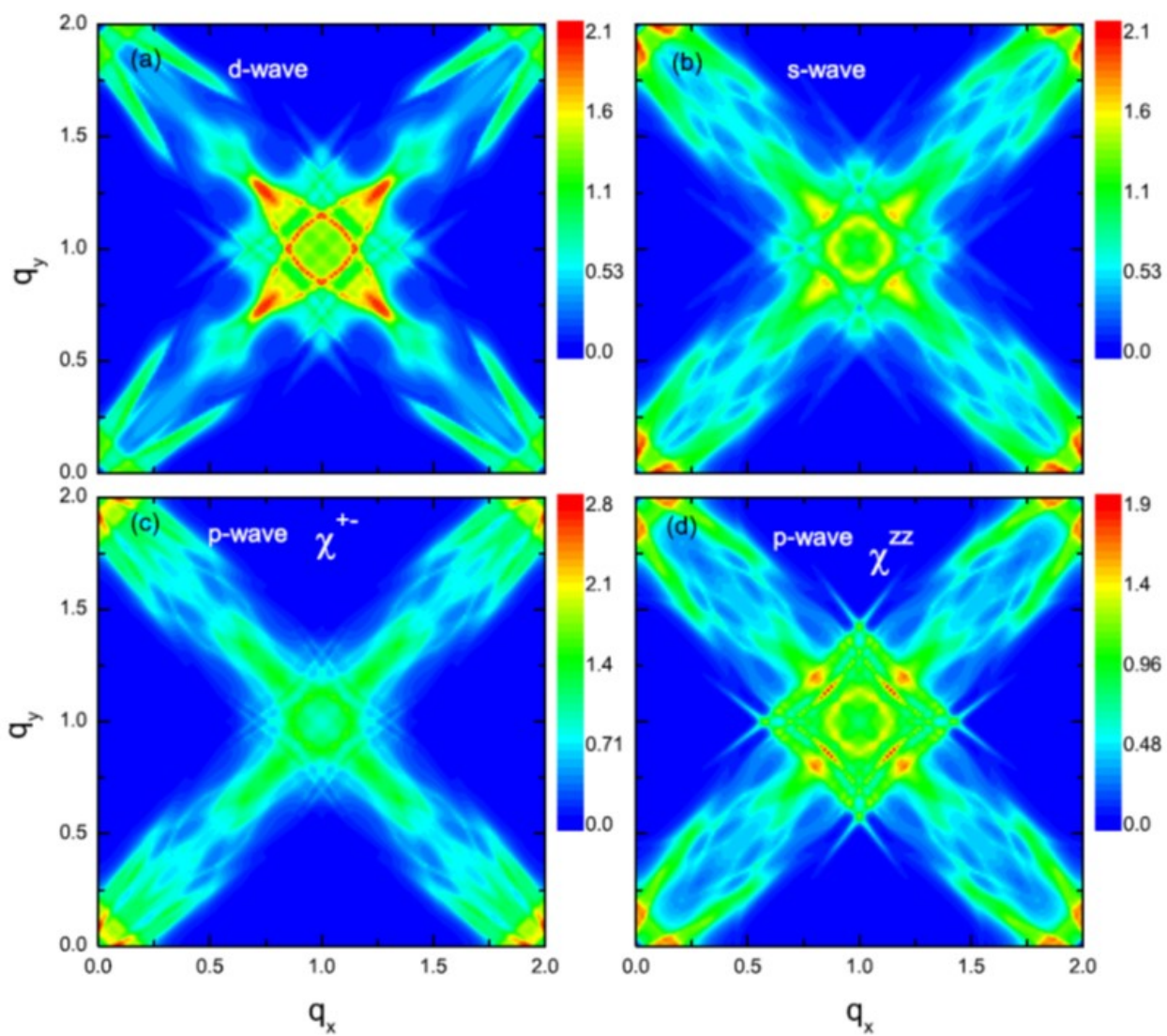

FIgURE 2.12: The intensity plots for imaginary parts of the spin susceptibility, Im $\chi(\mathrm{q}, \omega)=0.075$ for (a) $d$-wave, (b) extended $s$-wave, (c) $p$-wave $\chi^{+-}$and (d) $p$-wave $\chi^{z z} \cdot[89]$

is peaked around the doping level $x=0.5$, which is also consistent with experimental observations. [25] 


\section{Chapter 3}

\section{Neutron and Synchrotron X-ray Scattering}

\subsection{Introduction to Neutron Scattering}

Neutron scattering was employed as a tool to investigate structure of matter soon after its discovery in 1932 by James Chadwick [91], and at present, several neutron sources with specialized instruments around the world serve as giant microscopes to look into materials of a wide range such as bulk materials, thin films, multilayers and surfaces.

At reactor sources, neutrons are produced by fission of large unstable isotopes such as Uranium-235. The ejected high speed neutrons are then moderated/thermalized using moderators such as water, liquid hydrogen or methane to adopt a Maxwellian distribution of velocities. At spallation sources, neutrons are produced by bombardment of highly accelerated, intensed proton beam pulses onto a target as liquid $\mathrm{Hg}, \mathrm{Pb}$ or $\mathrm{W}$. A cartoon illustrating the two processes is shown in Fig. 3.1. At spallation sources, the resultant neutron beam is also pulsed unlike the continuous beam at reactors, which make them well suited for time of flight instruments. Excessive collisions during the 
moderation process might broaden the pulse of neutrons directed to instruments. Limitations are imposed on the size of moderators to avoid this which result in a residual of epithermal (>100 meV) neutrons. Therefore, the fission and spallation sources contain an overlap in temperature of neutrons produced, however, have differences in flux spectrum.

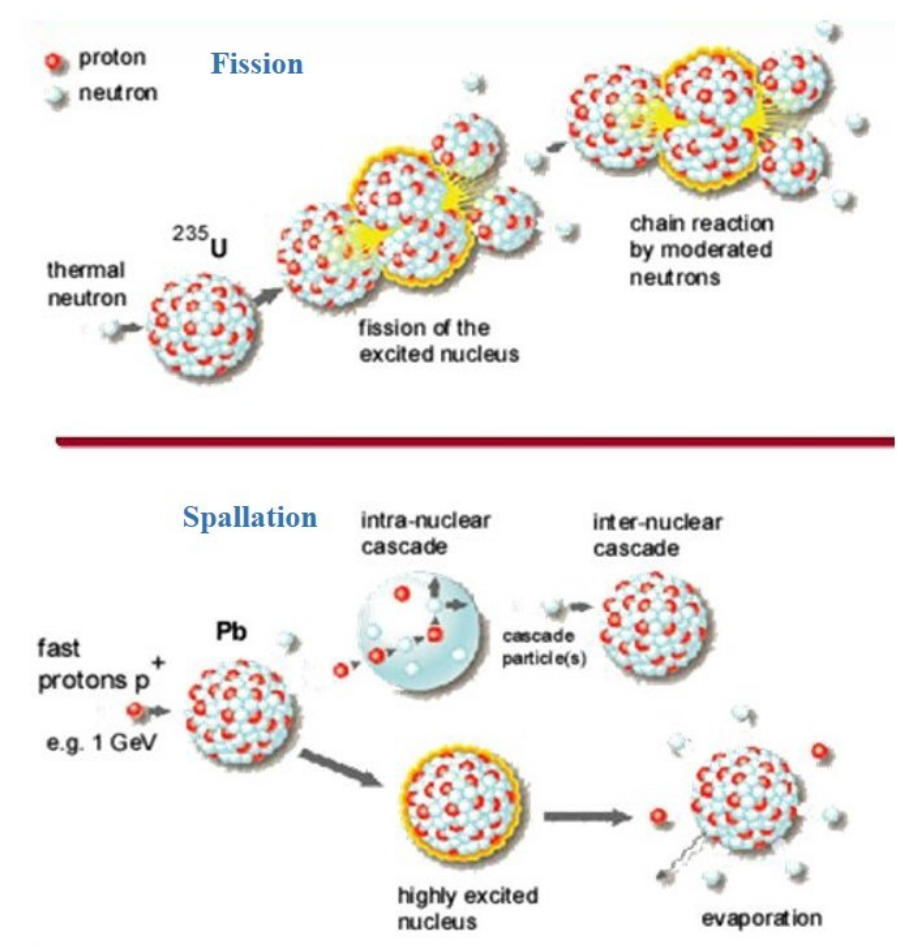

FiguRE 3.1: A cartoon illustrating how neutrons are produced by fission at reactor sources and by spallation at spallation sources.

With the fundamental properties of neutrons (shown in Table 3.1), the usefulness of neutrons in thermal range $(\sim 0.025 \mathrm{eV})$ is immense in investigating properties of matter. To look into the arrangement of atoms in a solid or a liquid, a scattering medium is needed in the range of inter atomic distances. Thermal neutrons having a de Broglie wavelength in the order of $\sim 10^{-10} \mathrm{~m}$, can serve this purpose. Also, the energy of thermal neutrons are in the order of excitations related to dynamical processes in matter, thus interaction of neutron with matter results in a significant change of energy in neutron, providing accurate measurements. Having zero net charge, neutrons have the ability to 
deeply penetrate into matter irrespective of the form of electron cloud and interfere with nuclei directly. The interaction between the neutron and the nucleus of an atom is known to be very short range $\left(\sim 10^{-13}\right)$ which is much less than the neutron wavelength. Thus the interaction is considered as point like. Therefore, it is an excellent tool to look into the bulk properties of the materials and convenient for experiments that need penetration of different sample environments such as cryostats and magnets. Furthermore, neutrons are able to detect the light atoms such as hydrogen or oxygen which are transparent to x-rays and also recognize different isotopes. The neutron has a non-zero magnetic moment, therefore can interact with unpaired electrons of the samples via dipole-dipole interactions. Thus it provides information of the spin arrangement and the unpaired electron density distribution.

TABLE 3.1: Fundamental properties of neutron [92]

\begin{tabular}{cc}
\hline Mass $(\mathrm{m})$ & $1.675 \times 10^{-27} \mathrm{~kg}$ \\
Charge & 0 \\
Spin & $\frac{1}{2}$ \\
Magnetic Dipole Moment $\left(\mu_{n}\right)$ & $-1.913 \mu_{N}$ \\
\hline
\end{tabular}

\subsection{Neutron Scattering Theory}

\subsubsection{Scattering geometry}

The conservation laws of momentum and energy applied to collisions are applicable for neutron scattering by matter as well.

$$
\begin{gathered}
\vec{Q}=\vec{k}_{i}-\vec{k}_{f} \\
\hbar \omega=E_{i}-E_{f}
\end{gathered}
$$


$\vec{Q}$ and $\hbar \omega$ refer to the momentum transfer and energy transfer of the neutron respectively. $\vec{k}$ is the wave vector of which the magnitude is determined by $k=2 \pi / \lambda$ where $\lambda$ is the neutron wavelength typiacally between $1 \AA$ and $10 \AA$. The scattering triangle is shown in Fig. 3.2. It depicts the magnitude and direction of $Q$ determined by the wave vectors of incident neutrons and scattered neutrons indicated with subscripts $i$ and $f$, respectively.
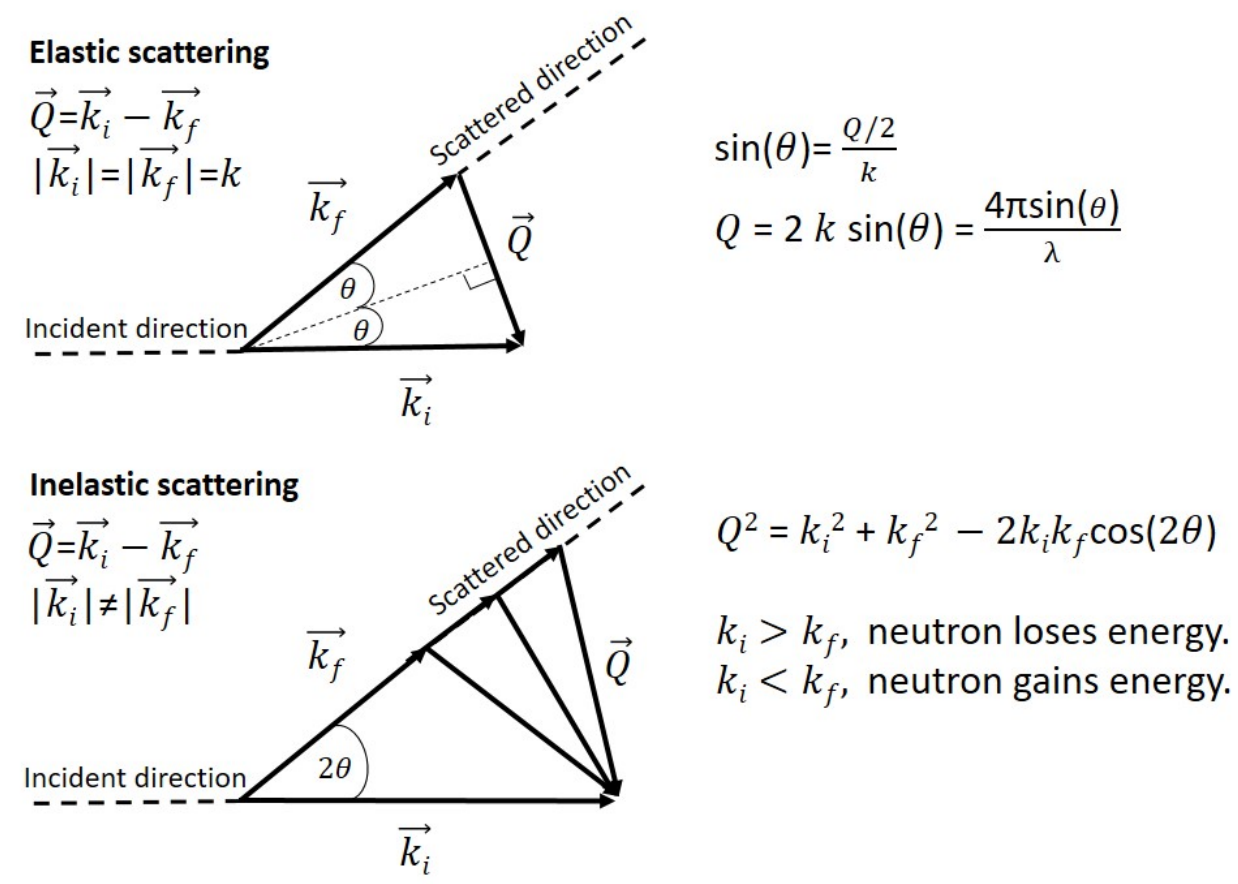

FIGURE 3.2: The scattering triangle with the incident wave vector $\vec{k}_{i}$, scattered wave vector $\vec{k}_{f}$ and transfered wave vector $\vec{Q}=\vec{k}_{i}-\vec{k}_{f}$.

The energy and momentum are related by the following equation where $m$ is the mass of the neutron and $\hbar$ is the reduced Planck constant.

$$
E=\hbar^{2} k^{2} / 2 m
$$

In elastic neutron scattering, magnitudes of the initial and final wave vectors are equal. Therefore, no energy is transfered to the crystal from the neutron. The momentum transfer can be written as a function of scattering angle, $\theta$, and neutron wavelength, $\lambda$ 
as follows.

$$
Q=4 \pi \sin (\theta) / \lambda
$$

When the neutrons are scattered by nuclei, the collisions will not necessarily be elastic.

The neutron can lose or gain energy during the scattering process making the magnitudes of initial and final wave vectors different. If the magnitude of the wave vector of the scattered neutron decreases, neutron loses energy, or, if it increases, neutron gains energy.

\subsubsection{Nuclear scattering cross-section}

In a neutron scattering experiment, the sample is hit with a beam of neutrons and the intensity of neutrons scattered by the sample is measured. This measured intensity is

often referred to as the partial differential cross-section, $\frac{d^{2} \sigma}{d \Omega d E}$, and it is defined as the rate of scattered neutrons into a solid angle of $d \Omega$ in the direction of scattered wave vector $\vec{k}_{f}$ with energy range between $E$ and $\mathrm{d} E$, normalized by the flux of beam $\phi$, as given by Eq. 3.5.

$$
\frac{d^{2} \sigma}{d \Omega d E}=\frac{\text { number of neutrons scattered per second into } d \Omega \& d E}{\phi d \Omega d E}
$$

This measured quantity is formulated into an expression for an ensemble of nuclei by Van Hove in 1954 [92, 93] and showed that this quantity can be expressed with timedependent correlations between the positions of pairs of atoms in the sample as shown in Eq. 3.6. 


$$
\frac{d^{2} \sigma}{d \Omega d E}=\frac{1}{h} \frac{k_{f}}{k_{i}} \sum_{l l^{\prime}} b_{l} b_{l^{\prime}} \int_{-\infty}^{\infty} d t\left\langle e^{-i \vec{Q} \cdot \overrightarrow{r_{l}}(0)} e^{i \vec{Q} \cdot \vec{r}_{l^{\prime}}(t)}\right\rangle e^{-i(E / \hbar) t}
$$

where the nucleus labeled as $l$ is at position $r_{l}$ at time zero, while the nucleus labeled $l^{\prime}$ is at position $r_{l}^{\prime}$ at time $t$. $b_{l}$ refers to the scattering length of the nucleus labeled $l$. The angular brackets indicate the thermodynamic average over the initial states of the sample.

Scattering length is different for isotopes of the same element. Even for the same isotope, scattering length depends on the relative orientation of the neutron and nuclear spin. The sum in Eq. 3.6 can be averaged over the initial spin states and the partial differential cross section can be written in terms of the the average value of scattering length $\bar{b}$, and the average value of square of the scattering length $\overline{b^{2}}$ as in Eq. 3.7.

$$
\frac{d^{2} \sigma}{d \Omega d E}=N \frac{k_{f}}{k_{i}} \bar{b}^{2} S(\vec{Q}, \omega)+N \frac{k_{f}}{k_{i}}\left(\bar{b}^{2}-\bar{b}^{2}\right) S(\vec{Q}, \omega)
$$

where

$$
S(\vec{Q}, \omega)=\frac{1}{2 \pi \hbar N} \sum_{l l^{\prime}} \int_{-\infty}^{\infty} d t\left\langle e^{-i \vec{Q} \cdot \overrightarrow{r_{l}}(0)} e^{i \vec{Q} \cdot \overrightarrow{r_{l^{\prime}}}(t)}\right\rangle e^{-i \omega t}
$$

where $N$ is the total number of nuclei in the sample. $S(\vec{Q}, \omega)$ is the scattering function which depends on the momentum transfer $Q$ and energy transfer $\hbar \omega$ which contains information about the microscopic properties of the sample. 
The first term in Eq. 3.7 corresponds to coherent scattering while second term corresponds to the incoherent scattering. $\sigma_{c o h}=4 \pi \bar{b}^{2}$ is called the coherent scattering cross section which provides information on atom $l$ and $l^{\prime}$ at time $t=0$ and $\mathrm{t}$, therefore, elastic coherent scattering gives information about equilibrium structure (Bragg scattering) and inelastic coherent scattering gives information about collective motions of atoms and their spins such as phonons and magnons.

In comparison, incoherent scattering provides information on atom $l$ at time $t=0$ and $\mathrm{t}$, in which neutrons are independently scattered from each nuclei and there is no interference between the scattering from different nuclei. $\sigma_{i n c}=4 \pi\left(\bar{b}^{2}-\bar{b}^{2}\right)$ is called the incoherent scattering cross section. Incoherent elastic scattering only contributes to the background which is same in all the directions in Q space.

\subsubsection{Bragg diffraction}

Diffraction involves elastic coherent scattering. When neutrons hit an ordered periodic arrangement of nuclei, the neutrons scatter from each nucleus with a definite phase relationship. Constructive interference occurs when the scattering waves from adjacent lattice planes have the same phase which happens when the path difference between the waves is equal to an integer multiple of the wavelength. This is known as the Bragg's Law, $2 d \sin (\theta)=n \lambda$, where $d$ is the distance between two scattering planes.

Ideally, in elastic coherent scattering, the energy and momentum of the incident and scattered neutrons are conserved. Therefore, $\left|\vec{k}_{i}\right|=\left|\vec{k}_{f}\right|$ and the coherent scattering term 
of equation 3.7 can be written as following.

$$
\frac{d^{2} \sigma}{d \Omega d E}=\frac{1}{2 \pi \hbar} \bar{b}^{2} \sum_{l l^{\prime}} \int_{-\infty}^{\infty} d t\left\langle e^{-i \vec{Q} \cdot \overrightarrow{r_{l}}(0)} e^{i \vec{Q} \cdot \overrightarrow{l_{l^{\prime}}}(t)}\right\rangle e^{-i(\omega) t}
$$

For a rigid Bravais lattice with a single element where the atoms are stationary, by taking the time average, the Eq. 3.9 can be written as,

$$
\frac{d^{2} \sigma}{d \Omega d E}=\bar{b}^{2} \delta(\hbar \omega) N \frac{(2 \pi)^{3}}{v_{0}} \sum_{\vec{\tau}} \delta(\vec{Q}-\vec{\tau})
$$

where $v_{0}$ is the unit cell volume, $\vec{\tau}$ is the reciprocal lattice vector and $N$ is the number of nuclei in the sample.

The elastic coherent scattering cross section is obtained by integrating with respect to energy.

$$
\frac{d \sigma}{d \Omega}=\bar{b}^{2} N \frac{(2 \pi)^{3}}{v_{0}} \sum_{\vec{\tau}} \delta(\vec{Q}-\vec{\tau})
$$

However, the atoms will not be stationary and oscillate around the equilibrium position, therefore, when taking the thermal averages, there will be some reduction in the intensity. This factor is known as Debye-Waller factor. It acts as a multiplying factor for each term in the expression of scattering cross section. Consider a lattice with more than one kind of atom per unit cell, where each has a different scattering cross section. Considering the Debye-Waller factor, coherent scattering cross section can be written as, 


$$
\begin{array}{r}
\left(\frac{d \sigma}{d \Omega}\right)=N \frac{(2 \pi)^{3}}{v_{0}} \sum_{\vec{\tau}} \delta(\vec{Q}-\vec{\tau})\left|F_{N}(\vec{\tau})\right|^{2} \\
F_{N}(\vec{\tau})=\sum_{j} \bar{b}_{j} e^{i \vec{\tau} \cdot \vec{r}_{j}} e^{-W_{j}}
\end{array}
$$

where $F_{N}(\vec{\tau})$ is the nuclear structure factor. $\vec{r}_{j}$ refers to the equilibrium position and $e^{-W_{j}}$ is the Debye-Waller factor containing thermal displacement information of $j^{\text {th }}$ atom. The scattering occurs when $\vec{Q}=\vec{k}_{i}-\vec{k}_{f}=\vec{\tau}$, and follows Eq. 3.4, which is the equivalent form of Bragg's law. In a diffraction experiment, the observed intensities of each Bragg peak position is proportional to the square of the structure factor convoluted by instrumental resolution function which are then analyzed to obtain the structural information of the system.

\subsection{Pair Distribution Function}

The pair distribution function (PDF) describes the probability distribution of finding inter-atomic correlations separated by a distance $r$ in real space. Unlike in the Bragg diffraction which assumes periodicity of unit cell and gives the information of the atoms in one unit cell, the PDF takes both Bragg and diffuse scattering into account and yields the structure beyond the unit cell size.

The expression for the scattering function given by Eq. 3.8 can be written in terms of time dependent microscopic particle density operator $\rho(\vec{r}, t)[94]$, 


$$
S(\vec{Q}, \omega)=\frac{1}{2 \pi \hbar N} \int_{-\infty}^{\infty} d t e^{-i \omega t} \int d \vec{r} d \overrightarrow{r^{\prime}} e^{-i \vec{Q} \cdot \vec{r}}\left\langle\rho\left(\overrightarrow{r^{\prime}}-\vec{r}\right) \rho\left(\overrightarrow{r^{\prime}}, t\right)\right\rangle
$$

and,

$$
\rho(\vec{r}, t)=\sum_{l} \delta\left\{\vec{r}-\vec{r}_{l}(t)\right\}
$$

where $\vec{r}_{l}(t)$ is the time dependent position of the $l^{t h}$ scattering nucleus.

The pair distribution function, $G(\vec{r}, t)$ can be written with particle density operator,

$$
G(\vec{r}, t)=\frac{1}{N} \int d \overrightarrow{r^{\prime}}\left\langle\rho\left(\overrightarrow{r^{\prime}}-\vec{r}, 0\right) \rho\left(\overrightarrow{r^{\prime}}, t\right)\right\rangle
$$

with

$$
\int d \overrightarrow{r^{\prime}} G(\vec{r}, t)=N
$$

where $\mathrm{N}$ is the total number of scattering nuclei.

Therefore,

$$
S(\vec{Q}, \omega)=\frac{1}{2 \pi \hbar} \int_{-\infty}^{\infty} d t e^{-i \omega t} \int d \vec{r} e^{-i \vec{Q} \cdot \vec{r}} G(\vec{r}, t)
$$


The pair distribution function, $G(\vec{r}, t)$, is the spatial and temporal Fourier transformation of the total structure function $S(\vec{Q}, \omega)[93,94]$.

The sample scattering amplitude $\psi(\vec{Q})$ relating to the real-space atomic density function $\rho(\vec{r})$ is defined as,

$$
\psi(\vec{Q})=\int d r \rho(\vec{r}) e^{-i \vec{Q} \vec{r}}
$$

Total scattering structure function $S(\vec{Q})$ is proportional to the sample scattering amplitude squared and can be written as[95],

$$
S(\vec{Q})=\frac{1}{N}|\psi(\vec{Q})|^{2}=\frac{1}{N} \iint d \vec{r} d \overrightarrow{r^{\prime}} \rho(\vec{r}) \rho\left(\overrightarrow{r^{\prime}}\right) e^{i \vec{Q}\left(\vec{r}-\overrightarrow{r^{\prime}}\right)}
$$

Eq. 3.20 can be also obtained by carrying out the energy integration of Eq. 3.14.

Taking the Fourier transformation of $\mathrm{S}(\vec{Q})[95]$,

$$
\rho(\vec{R})=\frac{1}{(2 \pi)^{3}} \int S(\vec{Q}) e^{i \vec{Q} \cdot \vec{R}} d \vec{Q}
$$

$$
\rho(\vec{R})=\rho_{0} g(\vec{R})
$$

where $\rho_{0}$ is atomic density and $g(\vec{R})$ is the probability of finding a bond length at $\vec{R}$. 
For isotropic amorphous materials, or powders, $S(Q)$ is isotropic, and angular variables can be integrated out as below.

$$
\begin{aligned}
\rho(\vec{R}) & =\frac{1}{(2 \pi)^{3}} S(Q) e^{i Q R \cos \theta} Q^{2} d \cos \theta d \phi d Q \\
& =\frac{1}{4 \pi^{2}} \int_{0}^{\infty} \int_{-1}^{1} S(Q) e^{i Q R \cos \theta} Q^{2} d \cos \theta d Q \\
\rho_{0} g(\vec{R}) & =\frac{1}{2 \pi^{2}} \int_{0}^{\infty} S(Q) \frac{\sin (Q R)}{Q R} Q^{2} d Q
\end{aligned}
$$

Due to the practical limitations, an infinite $Q$ is not reached and the integral is terminated at a finite $Q_{\max } . S(Q)$ approaches unity when $\mathrm{Q}$ is very large. Thus, with finite $Q_{\max }$ as the upper integration limit of Eq. $3.23, \rho(\vec{R})$ oscillates largely as a function of $Q_{\max }[95]$. To avoid this problem one can subtract the contribution from the average continuum which is customarily done by subtracting unity from $S(Q)$ and $g(R)$ which makes $[S(Q)-1] \longrightarrow 0$ at a large enough value of $Q_{\max } \cdot[95] \mathrm{A} Q_{\max }>30 \AA$ [96] is often considered sufficient to minimize these termination errors. Therefore, the standard expression is obtained as,

$$
\rho_{0}(g(R)-1)=\left(\frac{1}{2 \pi^{2} R}\right) \int[S(Q)-1] \sin (Q R) Q d Q
$$

and the pair distribution function, $G(R)$, can be written in terms of pair density function $\rho(R)$ as,

$$
G(R)=4 \pi R\left[\rho(R)-\rho_{0}\right]
$$


Thus, $G(R)$, can be defined as [97, 98],

$$
G(R)=\left(\frac{2}{\pi}\right) \int_{Q=0}^{Q_{\max }}[S(Q)-1] \sin (Q R) Q d Q
$$

The integrated area under the PDF peaks between two distances, $r_{1}$ and $r_{2}$, corresponds to the coordination number of atoms in the specified range of real space. If a particular peak corresponds to more than one type of atom, this quantity has to be weighted according to below equation.

$$
N_{i j}=\frac{4 \pi\langle b\rangle^{2} \int_{r_{1}}^{r_{2}} r^{2} \rho(r) d r}{2 C_{i} b_{i} b_{j}}
$$

where $\langle b\rangle$ is the average scattering length of the sample, $C_{i}$ is the chemical concentration of the central atom $i ; b_{i}$ and $b_{j}$ are the scattering lengths of atom $i$ and $j$ respectively. $N_{i j}$ is the number of pairs of atoms $i j$ defined by $r_{1}$ and $r_{2}$.

The correlated Debye model $[99,100]$ can be used to find the Debye temperature, $\theta_{D}$, from the extracted full width half maximum (FWHM), $\sigma_{i j}$, of the first PDF peak.

$$
\begin{aligned}
\sigma_{i j}^{2}=\frac{6 \hbar}{M \omega_{D}}[ & \left.\frac{1}{4}+\left(\frac{T}{\theta_{D}}\right)^{2} \Phi_{1}\right]-\frac{6 \hbar}{M \omega_{D}}\left[\frac{1-\cos \left(k_{D} r_{i j}\right)}{2\left(k_{D} r_{i j}\right)^{2}}\right. \\
& \left.+\left(\frac{T}{\theta_{D}}\right)^{2} \int_{0}^{\frac{\theta_{D}}{T}} \frac{\sin \left(\frac{k_{D} r_{i j} T x}{\theta_{D}}\right) / \frac{k_{D} r_{i j} T}{\theta_{D}}}{e^{x}-1} d x\right]
\end{aligned}
$$

$\sigma_{i j}$ in equation 3.28 is the FWHM of the PDF peak corresponding to the bond length between $i^{t h}$ and $j^{t h}$ atoms. The Debye wave vector is defined as $k_{D}=\left(6 \pi^{2} N / V\right)^{\frac{1}{3}}$ where $N / V$ is the number density, the Debye cutoff frequency $\omega_{D}=k_{B} \theta_{D} / \hbar$ where $k_{B}$ 
is the Boltzmann constant and $\Phi_{n}=\int_{0}^{\frac{\theta_{D}}{T}} x^{n}\left(e^{x}-1\right)^{-1} d x$, where $x$ is a dimensionless integration variable. The Debye temperature corresponds to the highest normal mode of vibration. A lower Debye temperature indicates a softer lattice.

\subsection{Synchrotron X-ray scattering}

A charged particle emits radiation when accelerating or decelerating. The synchrotron sources are based on this principle; radiation energy is emitted when the electrons are forced to change direction, and when the electrons are accelerated to high enough energies, the radiation of x-ray range is emitted. Synchrotron rings accelerate the electrons into energies as high as $\sim 7 \mathrm{GeV}[101]$ and the accelerated particles are bended and guided by magnets through an orbital path so that the direction is changed periodically. The emitted x-rays are guided to beamlines (instruments) which are designed to carry out different types of research.

Having a wavelength in the order of inter-atomic separations, x-rays serve as a useful probe in investigating structure of matter. Unlike the neutrons, x-rays interact primarily with the electron cloud surrounding atoms, therefore, the form factors are dependent on the atomic number. Hence, the light elements such as hydrogen and oxygen become transparent to x-rays. In addition, the x-ray wavelength is same as the spacial extent of the electron cloud, therefore, unlike the neutron the structure factor, the x-ray form factor is dependent on the momentum transfer, $\vec{Q}$ and falls off with increasing $Q$. Therefore, the neutron scattering length in Eq. 3.12 is replaced by the x-ray form factor of 
$j^{\text {th }}$ atom, $f_{j}$, as shown in Eq. 3.29.

$$
F_{N}(\vec{\tau})=\sum_{j} f_{j} e^{i \vec{\tau} \cdot \vec{r}_{j}} e^{-W_{j}}
$$

where $f_{j}=\int \rho_{j}\left(\overrightarrow{r_{j}}\right) e^{-2 \pi i \vec{Q} \cdot \overrightarrow{r_{j}}} d v . \quad \rho_{j}\left(\overrightarrow{r_{j}}\right)$ is the density of the electron cloud at a point determined by $\vec{r}_{j}$ whose origin is at the center of the $j^{\text {th }}$ atom. $v$ is the volume of the atom. [102]

Due to the primary difference of scattering method, an x-ray diffraction pattern and a neutron diffraction pattern of the same composition look different. Therefore, if data is available, simultaneous refinement of a structural model using both x-rays and neutrons, will give a unique structure.

\subsection{Neutron scattering instruments}

A wide range of neutron scattering instruments are available to study materials with varying interests. In this section, three mainly used techniques will be briefly discussed; neutron powder diffractometer, time of flight Laue diffractometer and four circle diffractometer.

\subsubsection{Neutron powder diffractometer (NPD)}

Neutron powder diffractometers have been widely used in both reactor sources and pulsed neutron sources to investigate crystal and magnetic structures under different sample environments as temperature, pressure, and magnetic field, and used in a variety of fields such as crystallography, mineralogy, geochemistry, solid-state physics, chemistry 
and material science. In neutron powder diffractometers at a reactor source, a single neutron wavelength is selected by a monochromater and the beam is directed to irradiate the powder samples. The scattered neutrons are counted by the detectors and recorded as a function of scattering angle, $2 \theta$. The neutron powder diffraction data consist of Bragg peak pattern which are scattering from lattice planes with different d-spacing. Fig. 3.3 shows the layout of a neutron powder diffractometer, D1B, which covers an angular range of $0.8^{\circ}$ to $128.8^{\circ}$ with position sensitive detectors.

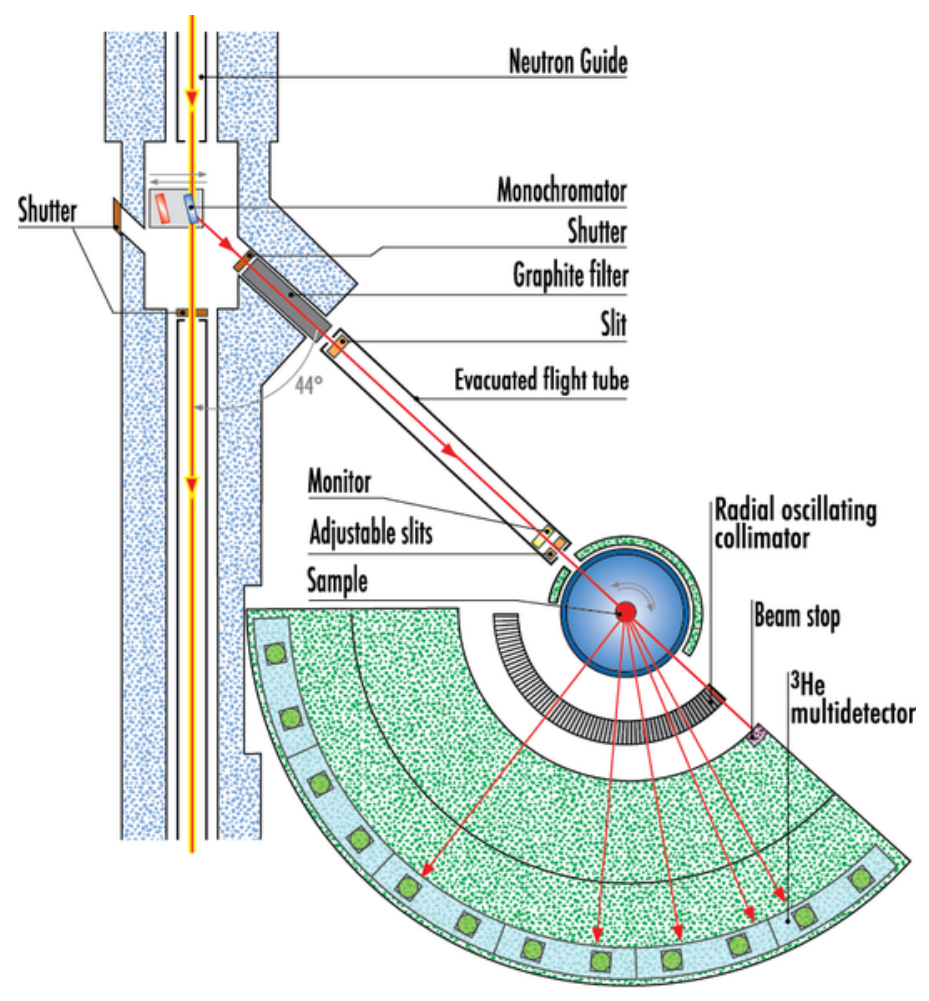

FiguRE 3.3: The layout of the neutron powder diffractometer, D1B.[103]

Powder diffractometers at neutron pulse sources use time of flight (ToF) technique. In Time of Flight technique, as the name implies, the energy or wavelength is measured as a function of time of flight. A white neutron beam hits the sample, scattered and detected by banks of detectors fixed at specific angles as shown in Fig. 3.4. The neutron flight path distance is known and the start time, and the arrival time at the detector of the each neutron is also recorded, therefore, the velocity of neutrons can be calculated. 
The sample position is kept fixed. This is one of the most efficient ways to use neutrons as there is no neutron loss due to the use of wide range of wavelengths, $\lambda$, and having access to large volume of reciprocal space between $1 / \lambda_{\min }$ and $1 / \lambda_{\max }$ simultaneously in a single measurement. In a powder diffractometer using time of flight technique, diffraction pattern is similar to what is obtained from a diffractometer at a reactor source. The difference is that the number of scattered neutrons are recorded as a function of time of flight instead of scattering angle 2 $\theta$. Fig. 3.5 shows the schematic diagram of the Nanoscale Ordered Materials Diffractometer (NOMAD) located at Oak Ridge National Laboratory which is a high-flux, medium-resolution diffractometer that uses time of flight technique. In this diffractometer, a large bandwidth of neutron energies and a wide detector coverage is used to obtain diffraction pattern up to a very large momentum transfer range which enables to perform structural determination of local order in crystalline and amorphous materials.

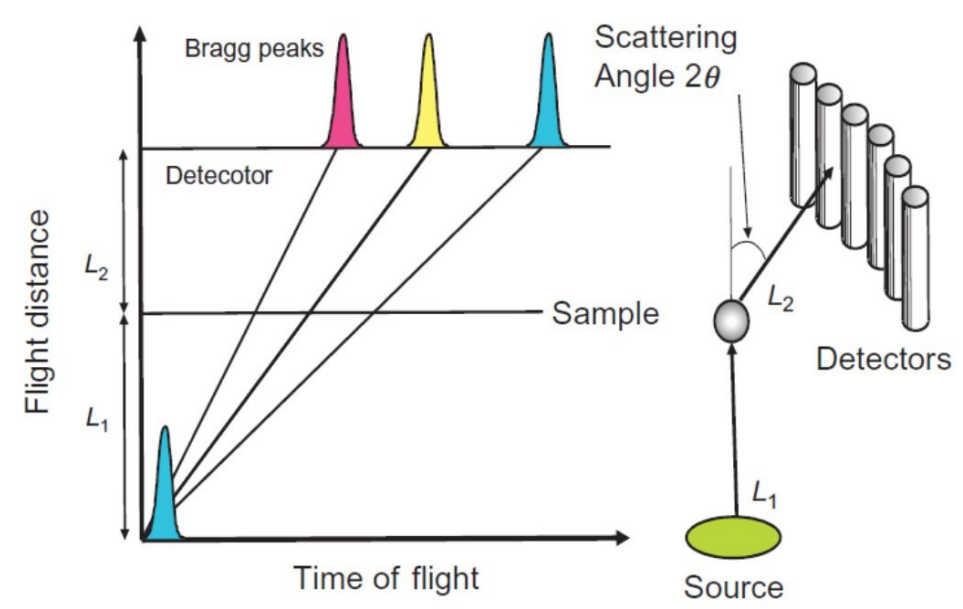

Figure 3.4: The principle of time of flight technique.[104] 


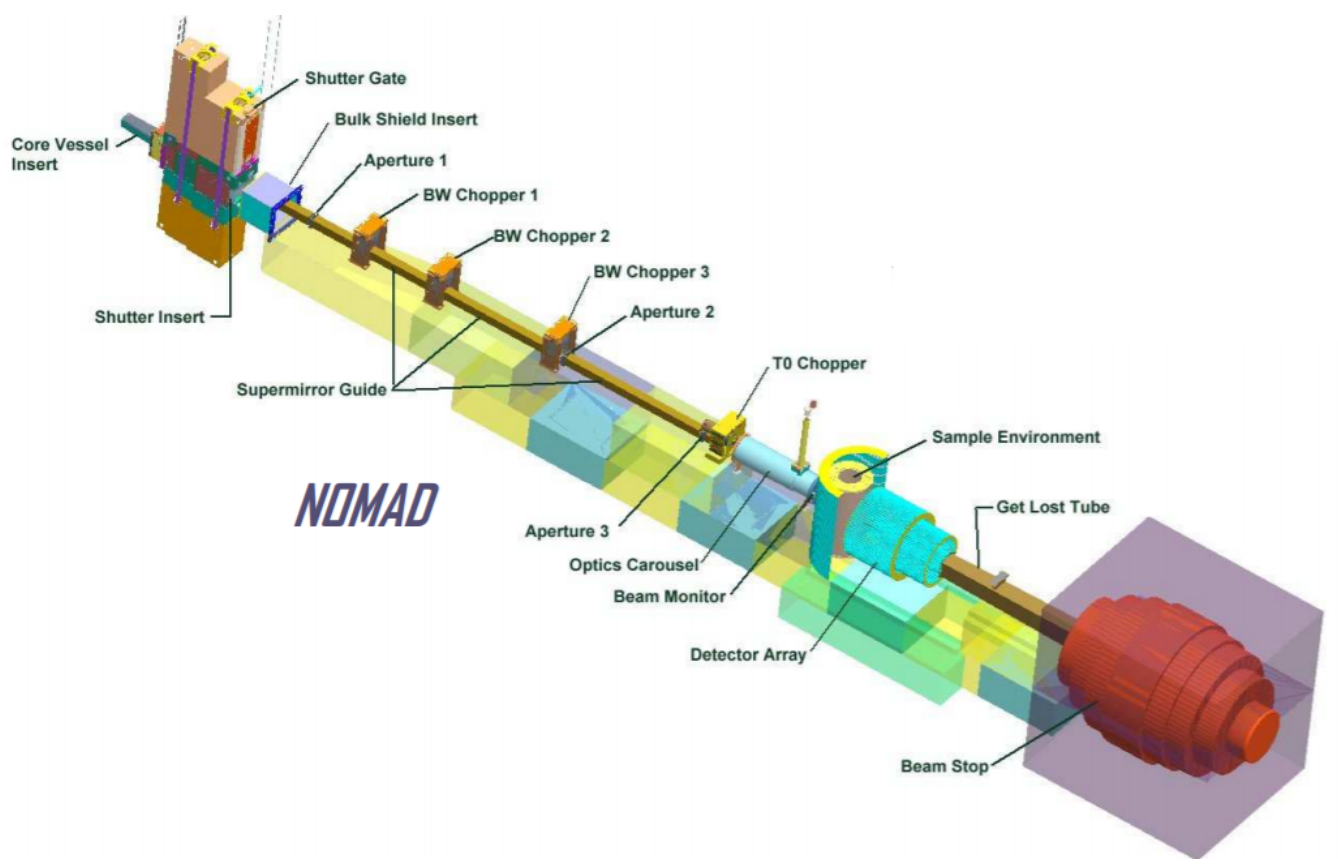

FiguRE 3.5: Schematic diagram of Nanoscale-Ordered Materials Diffractometer

\subsubsection{Time of flight Laue diffractometer}

When the time of flight method using a white neutron beam is implemented with an area detector, it is called ToFLD (time of flight Laue diffraction). It enables measurement of large volumes of reciprocal space in a single measurement with a single crystal sample. A group of reflections is measured at a single time using one 2D detector. Combining the different sets of data obtained from each detector, a large volume of reciprocal space can be investigated. Topaz, as shown in Fig. 3.6 is a high resolution Laue neutron singlecrystal diffractometer located at Oak Ridge National Laboratory that can be used to study sub-millimeter size crystals $(0.1-4 \mathrm{~mm})$ and capable of measuring a 3D volume of reciprocal space during each pulse. It has $20-160^{\circ} 2 \theta$ coverage in scattering plane and $+/-32^{\circ}$ out-of-plane. 

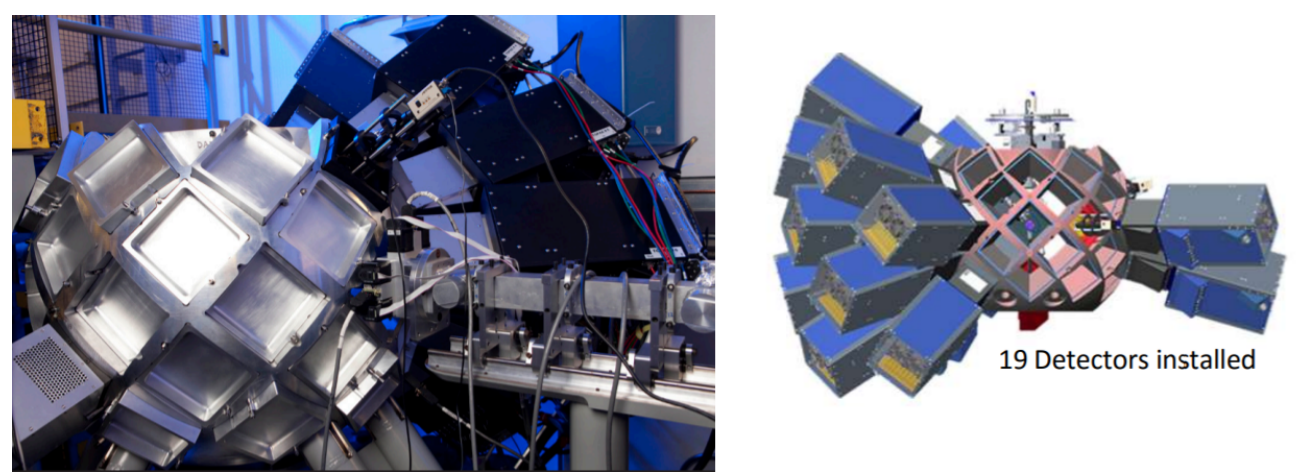

FiguRE 3.6: Topaz - high resolution Laue neutron single-crystal diffractometer located at Oak Ridge National Laboratory

\subsubsection{Four circle diffractometer}

Four circle diffractometers are widely used in single crystal analysis. Three crystal rotation axes, (Eulerian axes: $\chi, \phi$ and $\omega$ ) and the detector shaft $(2 \theta)$ as shown in Fig. 3.7 can be independently rotated. After the crystal is placed on the goniometer head in an arbitrary orientation, given the dimensions of the unit cell of the crystal, the crystal orientation will be determined in terms of four angles of the diffractometer. After the initial orientation, diffractometer can access $(h k l)$ reflections in 3-dimentional Q space. The diffracted rays at the correct orientation satisfying the Bragg conditions are then collected by the detector. Most of the four circle diffractometers consist of $2 \mathrm{D}$ area detectors.

\subsection{Data analysis}

\subsubsection{Powder diffraction data analysis}

In a polycrystalline or powder sample there are many tiny single crystal grains randomly oriented. Thus, in any orientation there will be always some grains satisfying the Bragg's law. A powder diffraction data set consists of normalized neutron counts 


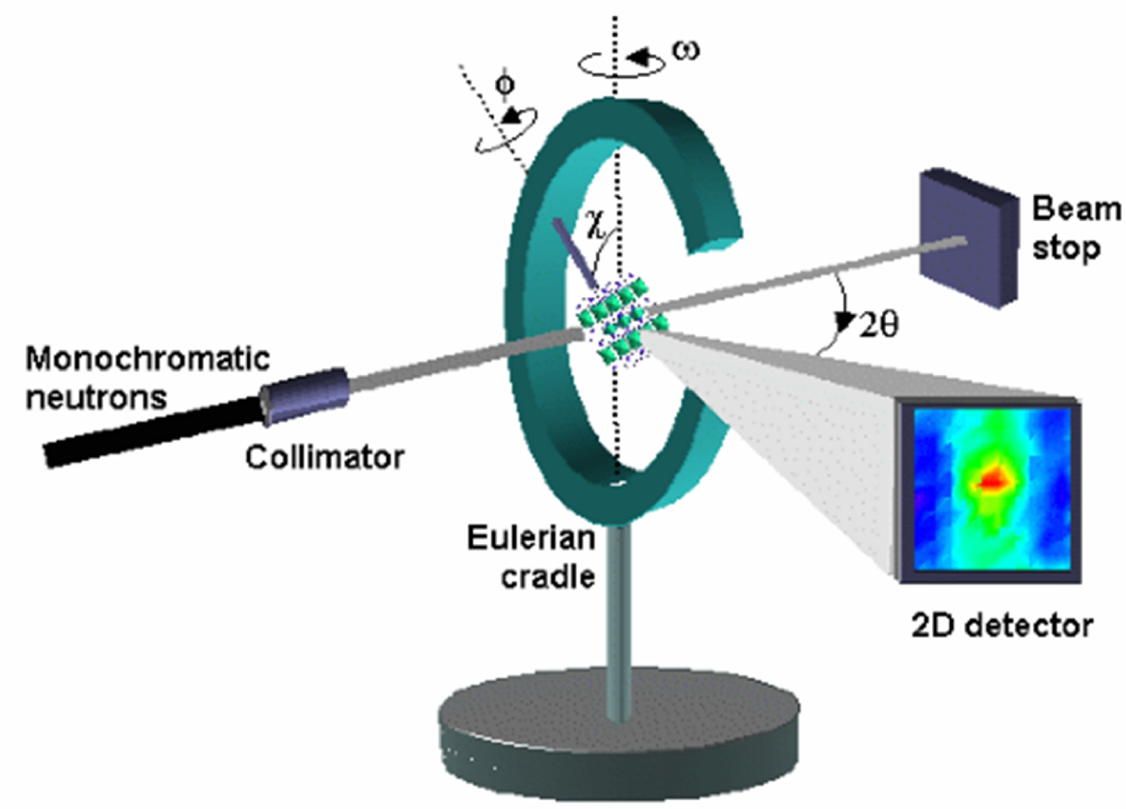

FiguRE 3.7: The layout of a four circle diffractometer.[105]

observed as a function of $2 \theta$ or time of flight (ToF) depending on whether it is measured with a powder diffractometer at a reactor source or at a pulse neutron source respectively. $2 \theta$ or ToF can be converted to momentum transfer $Q$ or $d$-spacing. Bragg peaks appear when the momentum transfer is equal to the reciprocal lattice vector, and the strength of the Bragg peaks are dependent on the atomic positions. In Rietveld analysis that uses a least-squares refinement procedure, a structural model is refined so that it will match with the measured powder diffraction pattern. In the work presented, powder diffraction refinement was carried out using the General Structure Analysis System (GSAS) software.[106, 107] The starting values of lattice parameters, atomic positions, thermal parameters, occupancy, the peak profile (parameters that determine the Bragg peak shape) and background coefficients are first entered in to the software. The profile parameters and diffractometer constants are provided with the parameter file of each instrument used. First, lattice parameters and background coefficients are refined keeping other parameters constant. Secondly, the atomic positions, peak profile parameters and 
thermal parameters are refined step by step. Finally, the occupancy of the atoms can be refined. The optimized parameters for each of the above mentioned parameters were obtained by performing the refinement in an iterative manner to achieve the best fit to the data. The model assessment is done graphically looking at the observed and calculated intensities and statistically calculating weighted profile $R$ factor $\left(R_{w p}\right)$ or goodness of fit, $\chi^{2}$ which are defined as in Eq. 3.30-3.32.[108]

$$
R_{w p}^{2}=\sum w\left(I_{c a l}-I_{o b s}\right)^{2} / \sum w\left(I_{o b s}\right)^{2}
$$

where $I_{o b s}$ and $I_{o b s}$ are the observed and calculated intensities, respectively. The weight labelled as $w$ is $1 / \sigma^{2}\left[I_{o b s}\right]$ where $\sigma^{2}\left[I_{o b s}\right]$ is the uncertainty estimate of $I_{o b s}$.

$$
\chi^{2}=(1 / N) \sum\left(I_{c a l}-I_{o b s}\right)^{2} / \sigma^{2}\left[I_{o b s}\right]
$$

$$
\chi^{2}=\left(R_{w p} / R_{e x p}\right)^{2}
$$

where the expected $R$ factor, $R_{e x p}=\sqrt{N / \sum w\left(I_{o b s}^{2}\right)}$ and $N$ is the total number of points assessed in reciprocal space.

\subsubsection{Pair density function analysis and modelling}

In diffraction data analysis, only the positions and relative intensities of the Bragg peaks are considered to refine a structure, which determine the long range structure 
of the sample. However, there is information in-between the Bragg peaks in the form of the diffuse scattering that are not used in diffraction data analysis. To look at the deviations from average crystal structure and local arrangement of atoms, we use Pair density function (PDF). PDF is obtained via Fourier transforming the normalized total scattering function, $S(Q)$ therefore, the information about local structure is preserved.

PDFgetN is the data analysis package that is used to process the ToF neutron diffraction data to obtain pair distributions functions (PDF's). Before fourier transforming the measured data to obtain the PDF, the data need to be corrected for background scattering, container scattering and incident spectrum. An empty instrument scan, an empty vanadium can measurement and a vanadium rod measurement were run respectively, in addition to the sample measurement. Using vanadium as the sample container, makes subtraction of its contribution easy as vanadium is an almost perfect incoherent scatterer. The data are corrected using Eq. 3.33.

$$
I=\frac{(S-B)-\alpha(C-B)}{V-B}
$$

where $\mathrm{S}$ is the sample scattering, $\mathrm{C}$ is the scattering from an empty sample container and $\mathrm{V}$ is the scattering from a vanadium sample that characterizes the spectrum. $B$ is the background spectra. The $\alpha$ factor corresponds to the absorption of the sample container.

As the next step, the absorption and multiple scattering corrections are carried out using the chemical composition of the sample, beam geometry and container dimensions. An option is available to apply Plazcek correction[109] to eliminate the inelastic scattering 
afterwards, however, perhaps it is not recommended because it might over-correct the low energy portion as this method assumes a high energy of neutron and its ballistic scattering by an atom. The normalized and corrected data of each detector bank will be combined to a single $S(Q)$ to yield PDF. Before blending the banks, it is important to check whether all spectra overlap with each other to confirm the normalization is acceptable for all banks. Using limits for each bank $\left(Q_{\min }\right.$ and $\left.Q_{\max }\right)$ the data are blended. These limits have to be adjusted until we get a good PDF with minimized oscillations and reliable peaks.

The next process is to model the experimental PDF. The first step is to calculate the PDF based upon the Rietveld analysis result and compare the differences, which indicate whether the local structure is deviated from the average structure. If differences exist, then the correlations in real space are changed such that it minimizes the discrepancies. The agreement factor, $A$, between the refined model and the experimental data is calculated at $n$ points equally apart in real space using Eq. 3.34.[96]

$$
A^{2}=\left(n \rho_{0}^{2}\right)^{-1} \sum_{i=1}^{n}\left[\rho_{\text {exp }}\left(r_{i}\right)-\rho_{\text {mod }}\left(r_{i}\right)\right]^{2}
$$

where $\rho_{0}$ is the atomic density of the unit cell.

\subsubsection{Single crystal data analysis}

The diffraction signature of a single crystal is a Bragg spots pattern while for polycrystalline samples they appear as rings on an area detector. In a single crystal diffraction experiment, the Bragg reflections are indexed using lattice parameters which are refined 
according to the recognized Bragg peak positions. The orientation matrix usually referred as UB matrix maps the crystal-based coordinates onto reciprocal space. Hence the integrated intensities are obtained as a function of reciprocal vector. The obtained intensity spectrum is refined using Shelx software[110] and the atom positions, thermal parameters and occupancies are refined to minimize the $R$ factor given in Eq. 3.35.

$$
R=\sum\left(F_{o b s}^{2}-F_{c a l}^{2}\right) / F_{o b s}^{2}
$$

where $F_{o b s}$ is the observed structure factor and $F_{c a l}$ is the calculated structure factor.

\subsection{Sample preparation and characterization}

Polycrystalline samples of $\mathrm{LaO}_{1-x} \mathrm{~F}_{x} \mathrm{BiS}_{2}(x=0$ and 0.5$)$ were prepared using standard solid state reaction method under ambient and high pressure. The samples of $x=0$ and high pressured $x=0.5$ were prepared at the National Institute for Materials Science (NIMS), Tsukuba, Japan. [25]. The polycrystalline samples of $x=0.2$ and $x=0.3$ were prepared at the University of Virginia, using a stoichiometric mixture of La (99.9\%), Bi (99.999\%), S (99.999\%), $\mathrm{LaF}_{3}(99.9 \%)$ and $\mathrm{La}_{2} \mathrm{O}_{3}$ (99.99\%) powders. They were ground, palletized and sintered at $800^{\circ} \mathrm{C}$ in an Ar filled quartz tube for $24 \mathrm{hrs}$. This procedure was repeated several times. Polycrystalline samples of $\mathrm{NdO}_{1-x} \mathrm{~F}_{x} \mathrm{BiS}_{2}(x=0.2$ and 0.4$)$ were also prepared at the National Institute for Materials Science, Japan. The samples were prepared using solid-state reaction using $\mathrm{Bi}$ grains, $\mathrm{Bi}_{2} \mathrm{~S}_{3}, \mathrm{Nd}_{2} \mathrm{O}_{3}, \mathrm{BiF}_{3}$ and $\mathrm{Nd}_{2} \mathrm{~S}_{3}$ powders. The stoichiometric amounts of each composition were ground, palletized and heated in an evacuated quartz tube at $700^{\circ} \mathrm{C}$ for 15 hours. 
The single crystals of $\mathrm{LaOBiS}_{2}$ and $\mathrm{LaO}_{0.7} \mathrm{~F}_{0.3} \mathrm{BiS}_{2}$ were grown using the $\mathrm{CsCl} / \mathrm{KCl}$ flux method and SEM/EDX was used to determine their stoichiometry and the bulk magnetization measurements were carried out using a Superconducting Quantum Interference Device (SQUID) magnetometer at University of Kentucky. $\mathrm{NdO}_{0.7} \mathrm{~F}_{0.3} \mathrm{BiS}_{2}$ and $\mathrm{PrO}_{0.7} \mathrm{~F}_{0.3} \mathrm{BiS}_{2}$ single crystals were also grown using the $\mathrm{CsCl} / \mathrm{KCl}$ flux method, in Japan, and the resistivity measurements were carried out using a physical property measuring system (PPMS) at University of Virginia. The crystals are flat, thin plate-like with the c-axis perpendicular to the growth direction.

\subsubsection{The transition temperature of $\mathrm{NdO}_{1-x} \mathrm{~F}_{x} \mathrm{BiS}_{2}$}

In $\mathrm{NdO}_{1-x} \mathrm{~F}_{x} \mathrm{BiS}_{2}$, the $x=0.2$ sample is non superconducting while the $x=0.4$ is superconducting with a $\mathrm{T}_{c}=5 \mathrm{~K}$ as indicated by bulk susceptibility data shown in Fig. 3.8.

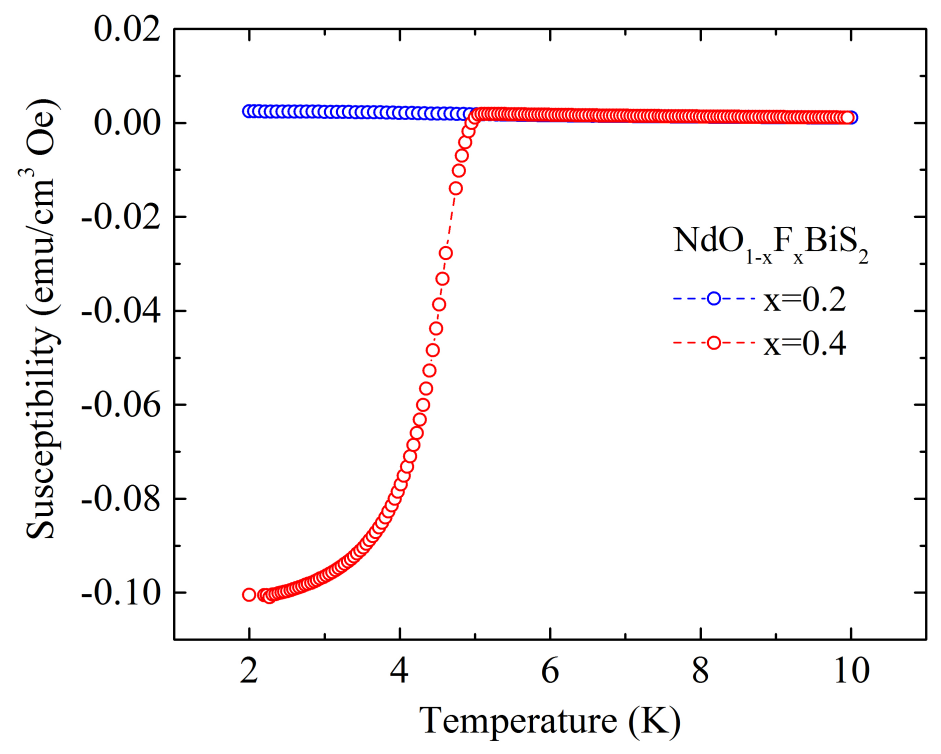

Figure 3.8: The susceptibility data of $\mathrm{NdO}_{1-x} \mathrm{~F}_{x} \mathrm{BiS}_{2}$ for non-superconducting $x=0.2$ and superconducting $x=0.4$. 
3.7.2 The transition temperature of $\operatorname{ReO}_{0.7} \mathrm{~F}_{0.3} \mathrm{BiS}_{2}(\mathrm{Re}=\mathrm{La}$ and $\mathrm{Nd})$ single crystals

The bulk magnetic susceptibility data of $\mathrm{LaO}_{0.7} \mathrm{~F}_{0.3} \mathrm{BiS}_{2}$ and resistivity data of $\mathrm{NdO}_{0.7} \mathrm{~F}_{0.3} \mathrm{BiS}_{2}$ are shown in Fig. 3.9 and Fig. 3.10, with a $\mathrm{T}_{c}$ of $\sim 2.5 \mathrm{~K}$ and $5 \mathrm{~K}$, respectively. Both samples are not high pressure annealed, therefore, it is clear that without high pressure annealing, $\mathrm{Re}=\mathrm{Nd}$ shows a higher transition temperature.

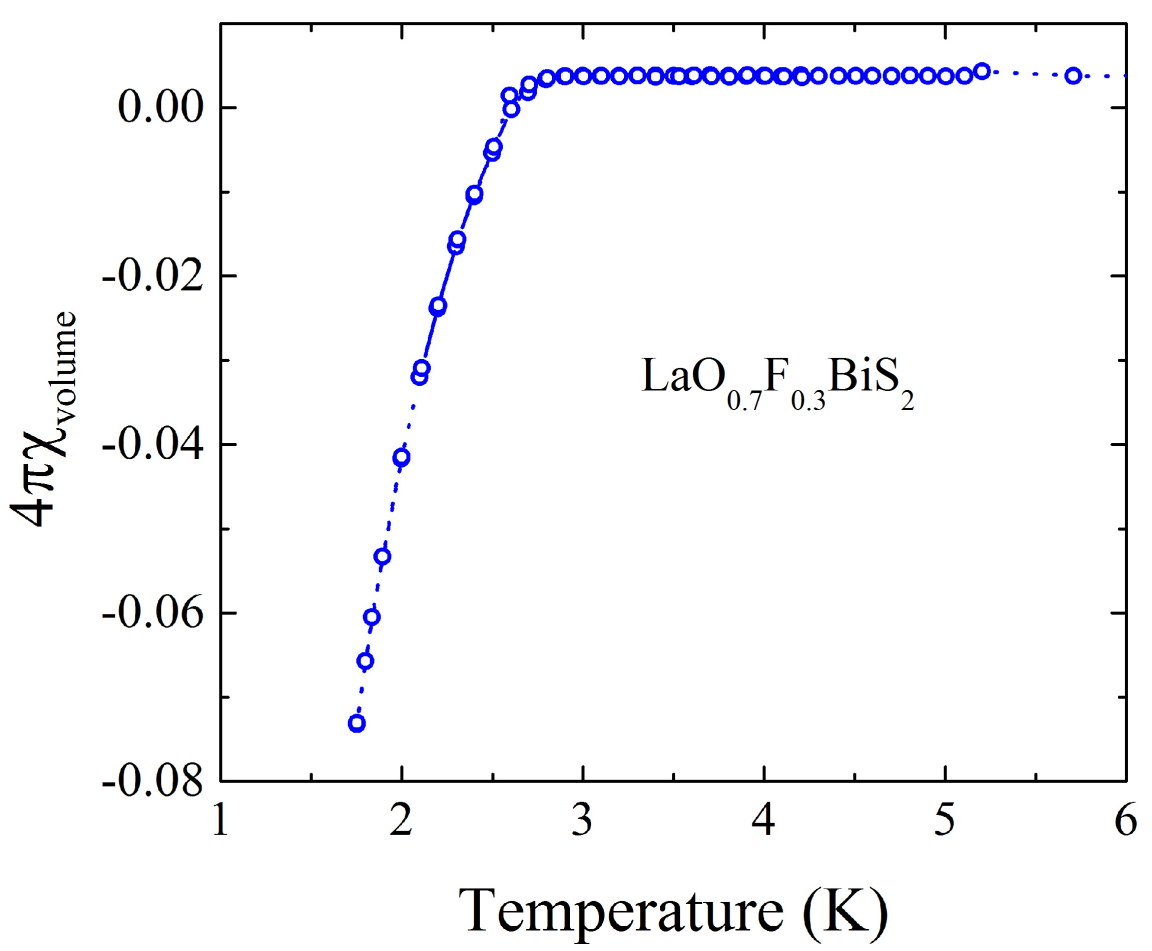

Figure 3.9: The bulk magnetic susceptibility data of the $\mathrm{LaO}_{0.7} \mathrm{~F}_{0.3} \mathrm{BiS}_{2}$ crystal showing a $\mathrm{T}_{c} \sim 2.5 \mathrm{~K}$. 


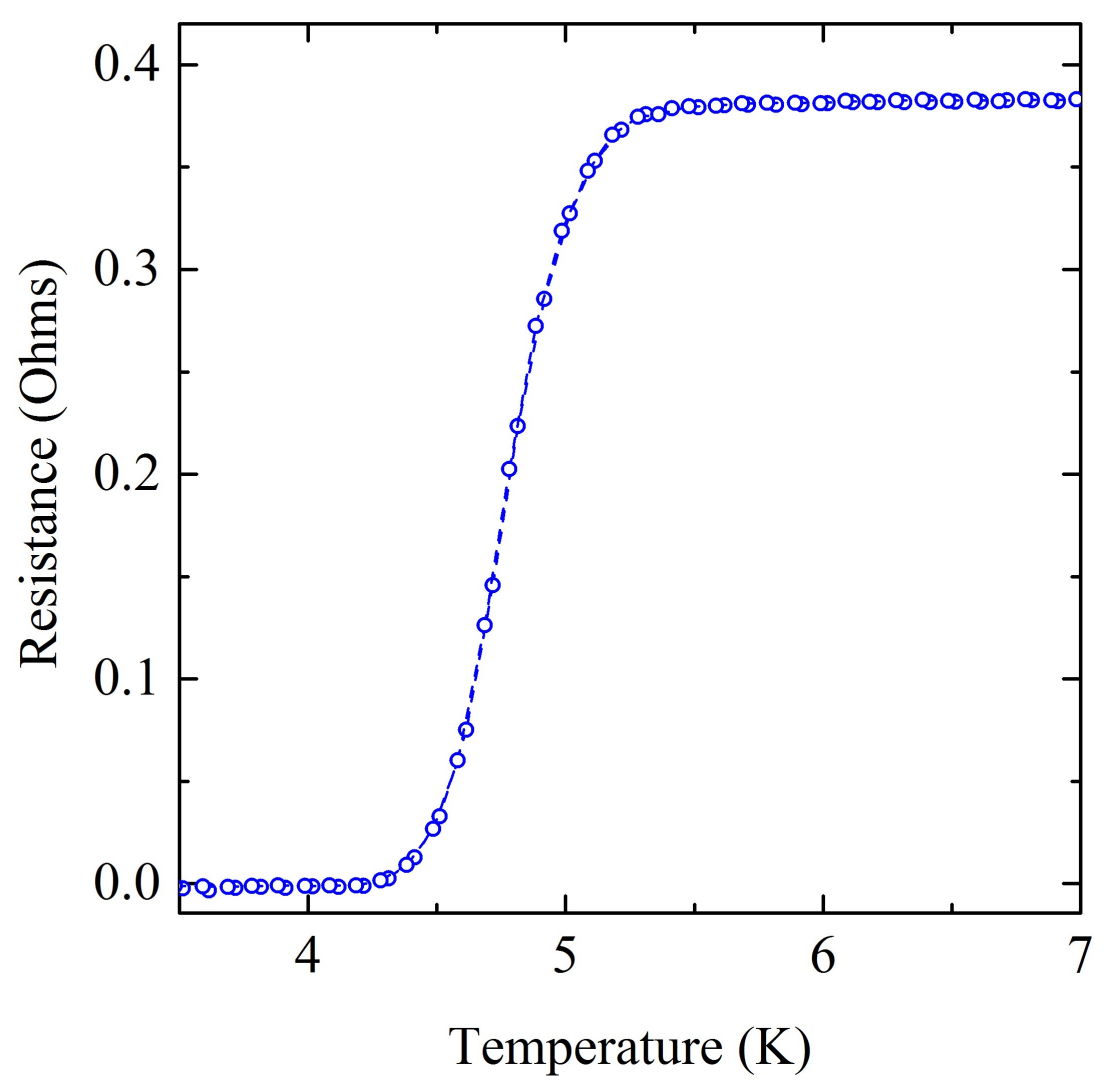

Figure 3.10: Resistivity data of $\mathrm{NdO}_{0.7} \mathrm{~F}_{0.3} \mathrm{BiS}_{2}$ crystal showing a $\mathrm{T}_{c} \sim 5 \mathrm{~K}$. 


\section{Chapter 4}

\section{The crystal structure and nature of local lattice distortions in superconducting $\mathrm{LaO}_{1-x} \mathbf{F}_{x} \mathrm{BiS}_{2}$}

\subsection{Introduction}

The low dimensional structures have been a fertile ground for exploring exotic superconductivity. Much of the recent interest in exotic superconductivity has been on high $\mathrm{T}_{c}$ cuprates or Fe based systems whose parent ground state is magnetic. The recently discovered class of non-magnetic superconductors with a similar layered structure containing $\mathrm{BiS}_{2}$ building blocks opened a novel path to investigate the superconducting mechanism of low dimensional superconductivity. In $\mathrm{BiS}_{2}$ superconductors, the dominant carriers are from the $\mathrm{Bi} 6 p$ orbitals that strongly hybridize with the $\mathrm{S} 3 p$ near the Fermi surface. [88] The $\mathrm{BiS}_{2}$ superconductors are possibly of the conventional type evidenced by a large electron phonon coupling constant, $\lambda \sim 0.8[17,18]$ but with strong electronic correlations evidenced by the large superconducting gap, indicating that the 
pairing is much stronger than the limit of a conventional phonon mediated superconductor. Included in the $\mathrm{BiS}_{2}$ class are the compounds of $\mathrm{ReO}_{1-x} \mathrm{~F}_{x} \mathrm{BiS}_{2}(\mathrm{Re}=\mathrm{La}, \mathrm{Nd}, \mathrm{Pr}$, Ce and $\mathrm{Yb})[25,26]$ and $\mathrm{Bi}_{4} \mathrm{O}_{4} \mathrm{~S}_{3}[8]$ that consist superconducting $\mathrm{BiS}_{2}$ planes. In these two families of $\mathrm{BiS}_{2}$ superconductors, crystal defects and atomic vacancies are common.

$\mathrm{LaO}_{1-x} \mathrm{~F}_{x} \mathrm{BiS}_{2}$ superconductor was discovered soon after the discovery of $\mathrm{Bi}_{4} \mathrm{O}_{4} \mathrm{~S}_{3}$ that led to recognizing of the common building block of $\mathrm{BiS}_{2}$. The parent compound of $\mathrm{LaO}_{1-x} \mathrm{~F}_{x} \mathrm{BiS}_{2}$, i.e $x=0$ is a band insulator with a gap of $\approx 0.8 \mathrm{eV}[15,88]$ as shown in Fig. 4.1. It has a tetragonal crystal structure with the nominal space group of $P 4 / \mathrm{nmm}$.

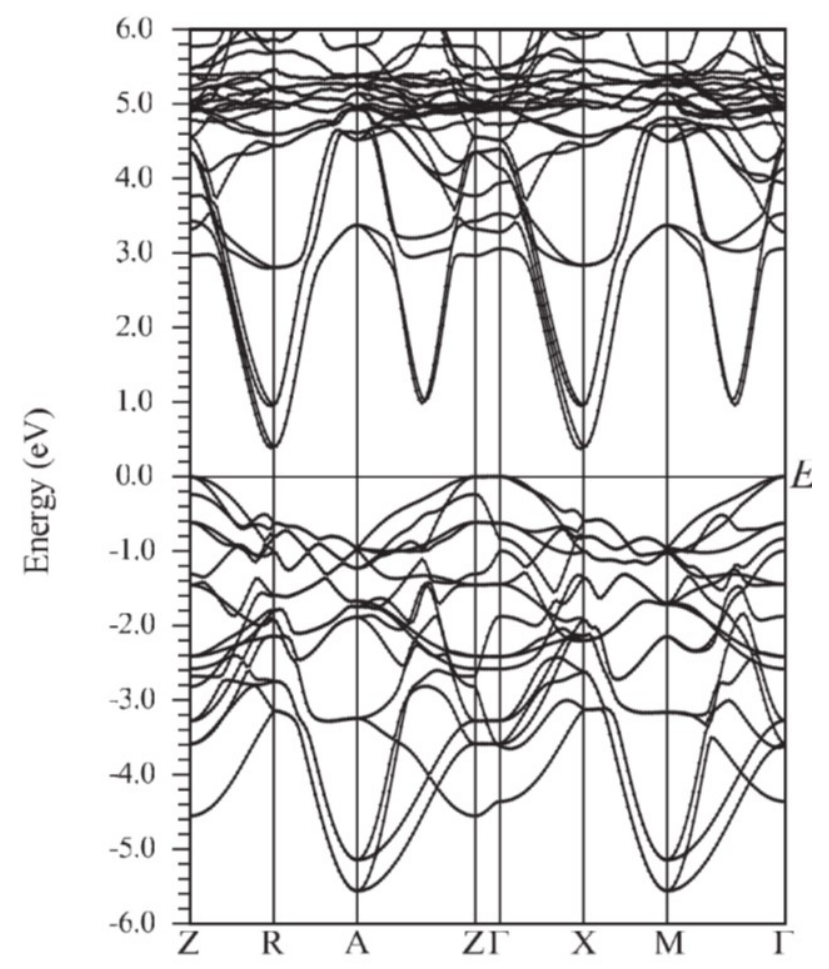

Figure 4.1: The band structure of $\mathrm{LaOBiS}_{2} \cdot[88]$

The superconductivity in $\mathrm{LaOBiS}_{2}$ is induced by fluorine doping at oxygen sites which introduces electron carriers. Upon fluorine doping, superconductivity appears in $\mathrm{LaO}_{1-x} \mathrm{~F}_{x} \mathrm{BiS}_{2}$ by $x=0.2$ and vanishes by $x=0.7$ [111] as shown in Fig. 4.2. 


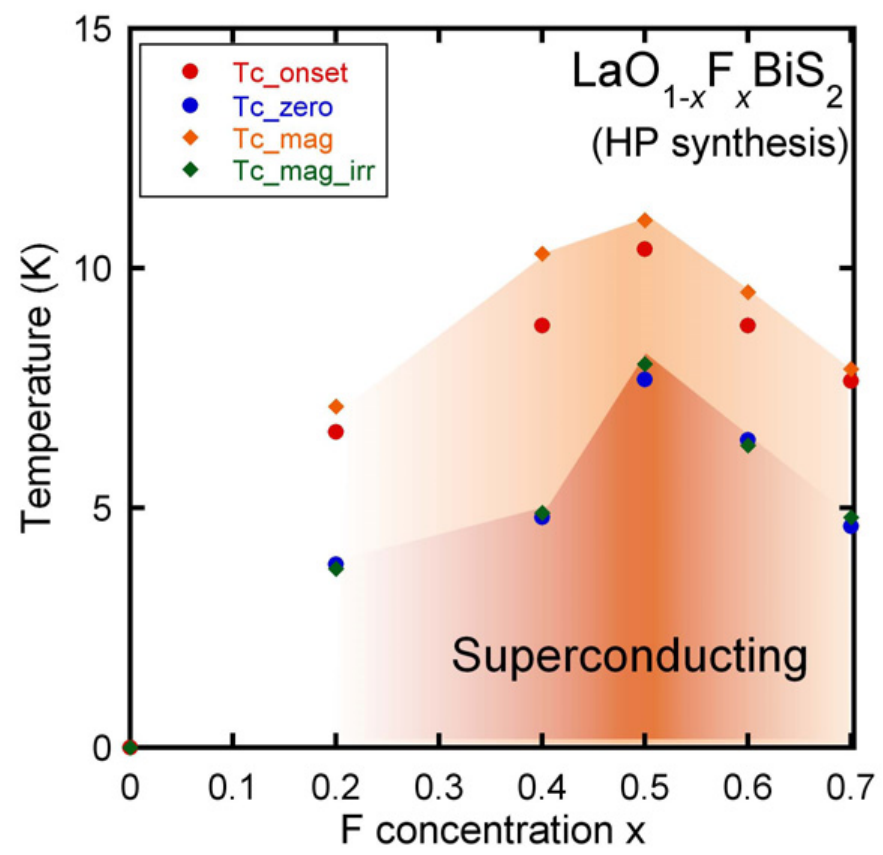

Figure 4.2: Phase diagram of $\mathrm{LaO}_{1-x} \mathrm{~F}_{x} \mathrm{BiS}_{2}$ prepared under high pressure annealing.[111]

The superconducting transition is enhanced under high pressure annealing and the highest $\mathrm{T}_{c}$ of $\sim 10 \mathrm{~K}$ is observed in the $x=0.5$. $[25,111]$ The specific role of high pressure annealing in the superconducting mechanism is not fully understood yet, however, it has been associated with the appearance of disorder in the crystal structure. Crystallinity usually becomes better with high pressure annealing in superconducting structures and gives rise to higher superconducting temperatures. However, $\mathrm{BiS}_{2}$ class shows a peculiar response to high pressure annealing. Its $\mathrm{T}_{c}$ is maximized with high pressure, but with induced crystal disorder. The $\mathrm{T}_{c}$ reaches $10 \mathrm{~K}$ with compromised crystallinity. Therefore, the nature of crystal disorder plays an important role in the superconducting mechanism of $\mathrm{BiS}_{2}$ superconductors and central towards enhancing transition temperature. Evidence for the disorder is observed in the Bragg peak broadening and the uniaxial strain that develops along the $c$ axis with high pressure annealing.[64] However, the connection of superconductivity to the high pressure annealing is not clarified yet. Also coupled to the disorder is the buckling of the $\mathrm{BiS}_{2}$ planes. Previous studies suggested 
that the $\mathrm{BiS}_{2}$ plane is buckled for low doping levels of $x$, while the buckling becomes almost flat by $x \sim 0.5[15,112]$ when $\mathrm{LaO}_{1-x} \mathrm{~F}_{x} \mathrm{BiS}_{2}$ reaches its maximum $\mathrm{T}_{c}$ as shown in Fig. 4.3.

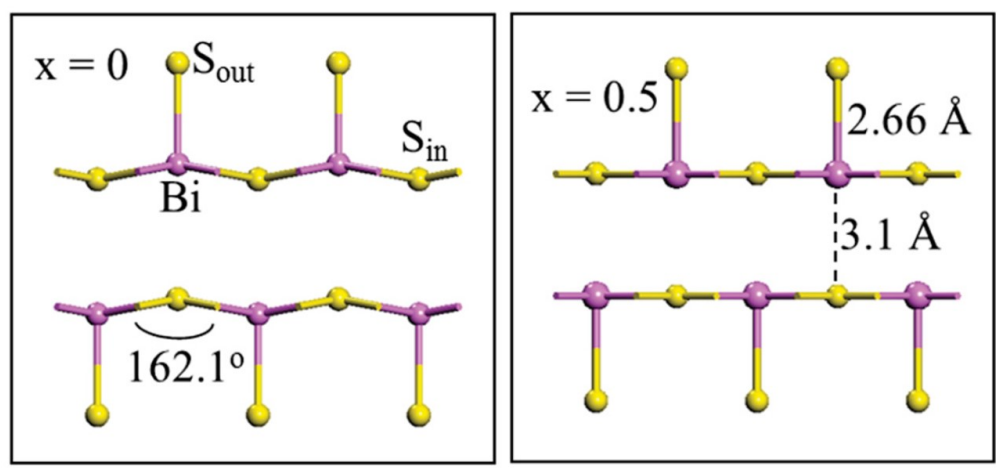

Figure 4.3: $\mathrm{BiS}_{2}$ plane of $\mathrm{LaO}_{0.5} \mathrm{~F}_{0.5} \mathrm{BiS}_{2}$ at $\mathrm{x}=0$ and 0.5.[15]

First principles calculations showed that the topology of Fermi surface changes upon electron doping with fluorine. In $\mathrm{LaOBiS}_{2}$, the most unstable phonon arises at $\Gamma$ point, however upon fluorine doping, the phonon instability arises along the entire line $(q, q, 0)$ with the most unstable phonon at $M$ point and $(\pi / 2, \pi / 2)$.[15] The phonon softening around $M$ point is associated with rather unusual structural and dynamical properties such as the development of a charge density wave instability and strong electron-phonon coupling that correspond to large-amplitude in-plane displacements of the sulfur atoms.

Spin orbit (SO) coupling has a considerable effect on the electronic band structure around the $\mathrm{X}$ point of the Brillouin zone as shown in Fig. 4.4. Without SO coupling, four bands cross below $\mathrm{E}_{F}$ in the optimally doped composition, $x \sim 0.5$. SO coupling 'pushes' two bands above so that only two bands cross below $\mathrm{E}_{F}$ with $\mathrm{SO}$ coupling. SO coupling originates from the inversion asymmetry and creates an effective magnetic field that lifts the spin degeneracy[33]. The inversion asymmetry could be driven by 
atomic site asymmetry even without breaking the crystal symmetry. This phenomenon is known as Dresselhaus effect in bulk 3D materials and Rashba effect in 2D materials.

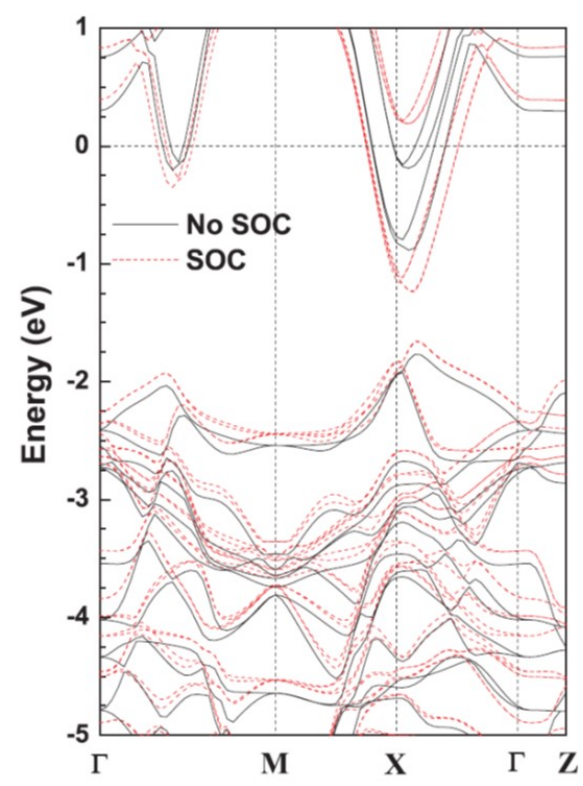

Figure 4.4: Band structure of $\mathrm{LaO}_{0.5} \mathrm{~F}_{0.5} \mathrm{BiS}_{2}$ with and without spin orbit coupling.[17]

The question whether $\mathrm{LaO}_{1-x} \mathrm{~F}_{x} \mathrm{BiS}_{2}$ is a conventional phonon superconductor or a new class of phonon superconductors, still remains. Given the intrinsic nature of the structural disorder and its strong relation to the superconductivity, precise analysis of the crystal structure is vital to understand the superconducting mechanism of $\mathrm{LaO}_{1-x} \mathrm{~F}_{x} \mathrm{BiS}_{2}$. To this end, we carried out neutron powder diffraction experiments to determine the crystal structure, pair density function analysis to investigate the subtle nano-scale atomic movements, and calculated Debye temperatures to determine the rigidity of the lattice. 


\subsection{Crystal structure of $\mathrm{LaO}_{0.5} \mathrm{~F}_{0.5} \mathrm{BiS}_{2}$}

The neutron diffraction data collected for $\mathrm{LaO}_{1-x} \mathrm{~F}_{x} \mathrm{BiS}_{2}(x=0,0.2,0.3$ and 0.5$)$ were analyzed in reciprocal space using Rietveld refinement. The width of the Bragg peaks are instrumental resolution limited. The Bragg diffraction pattern and the corresponding refined models of parent sample at $6 \mathrm{~K}$ and the doped samples at $2 \mathrm{~K}$ are depicted in Fig. 4.5 with black crosses representing the data points. The red line represents the calculated model, with Bragg peak positions represented with magenta short vertical lines. The difference between the refined model and the data is represented with a blue solid line. The diffraction patterns of all four compositions are well reproduced by the centrosymmetric space group of $P 4 / \mathrm{nmm}$. The crystal structure remains tetragonal with peak broadening at higher doping levels. Peak broadening is distinctly pronounced in high pressure (HP) annealed $x=0.5$ which is the only superconducting sample in this case. It is perplexing how the low crystallinity and distortions are conducive to superconductivity of this system. Previous studies showed that the broadening affects Bragg peaks with non zero $l$ indices while the Bragg peaks with $l=0$ are much sharper.[113] Fig. 4.6 is a comparison of two nuclear Bragg peaks of the parent and superconducting $x=0.5$. In the (114) where $l$ index is non zero, the Bragg peak is quite broad in HP annealed $x=0.5$ compared to the parent sample. In (110) Bragg peak, no broadening was observed upon doping. 


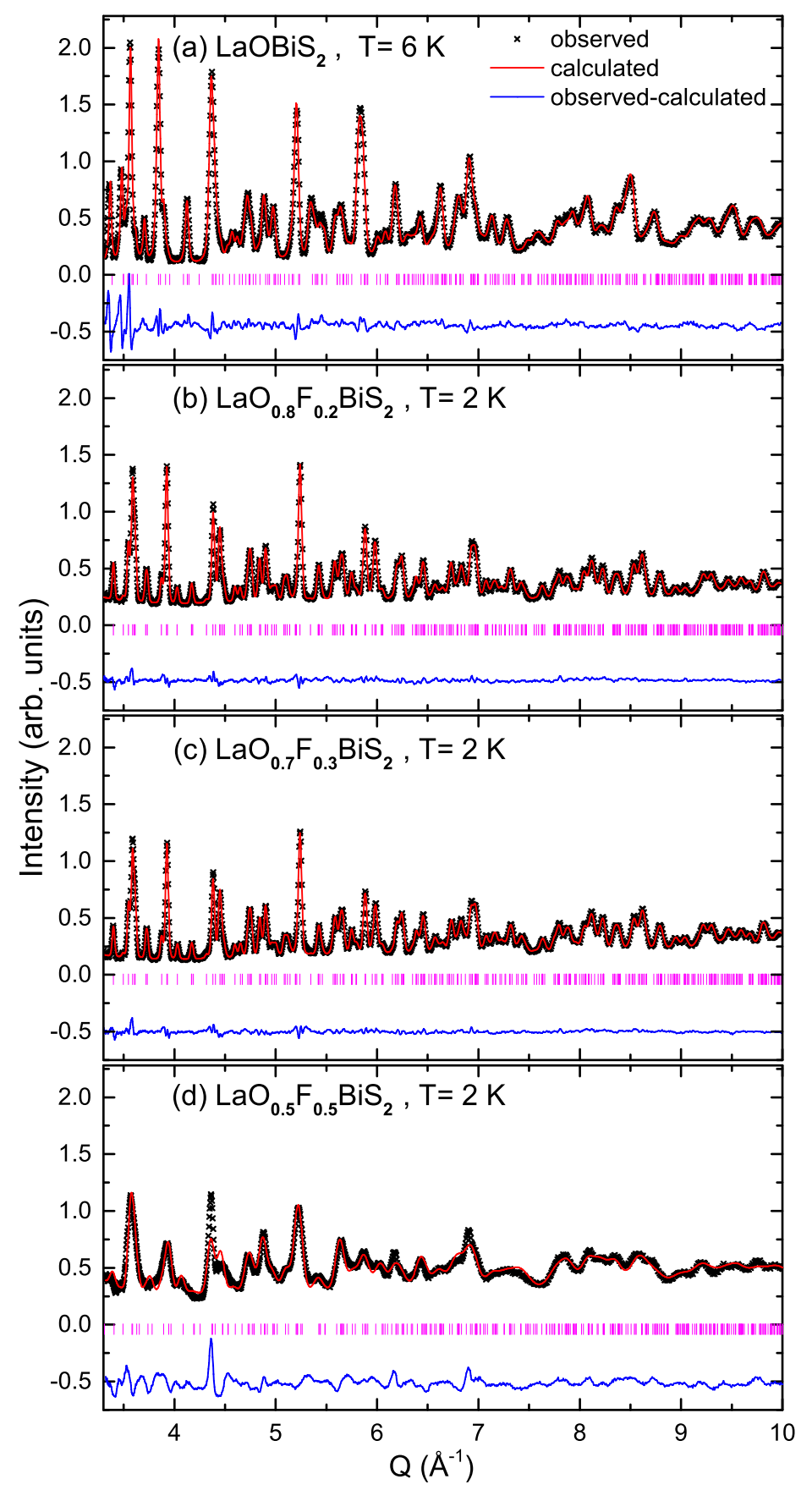

Figure 4.5: The neutron powder diffraction data of (a) $\mathrm{LaOBiS}_{2}$ at $6 \mathrm{~K}$, (b) $\mathrm{LaO}_{0.8} \mathrm{~F}_{0.2} \mathrm{BiS}_{2}$ at $2 \mathrm{~K}$, (c) $\mathrm{LaO}_{0.7} \mathrm{~F}_{0.3} \mathrm{BiS}_{2}$ at $2 \mathrm{~K}$, and (d) $\mathrm{LaO}_{0.5} \mathrm{~F}_{0.5} \mathrm{BiS}_{2}$ ( $\mathrm{HP}$ annealed) at $2 \mathrm{~K}$. Black crosses represent data points. Red and blue solid lines represent the calculated intensity and the difference between observed and calculated intensities, respectively. The magenta short vertical lines show the Bragg positions. $[114]$ 
(a)

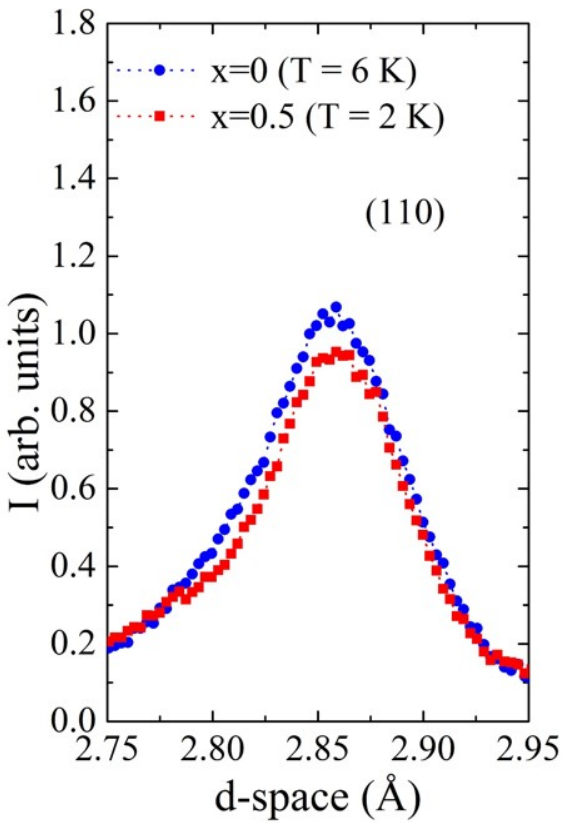

(b)

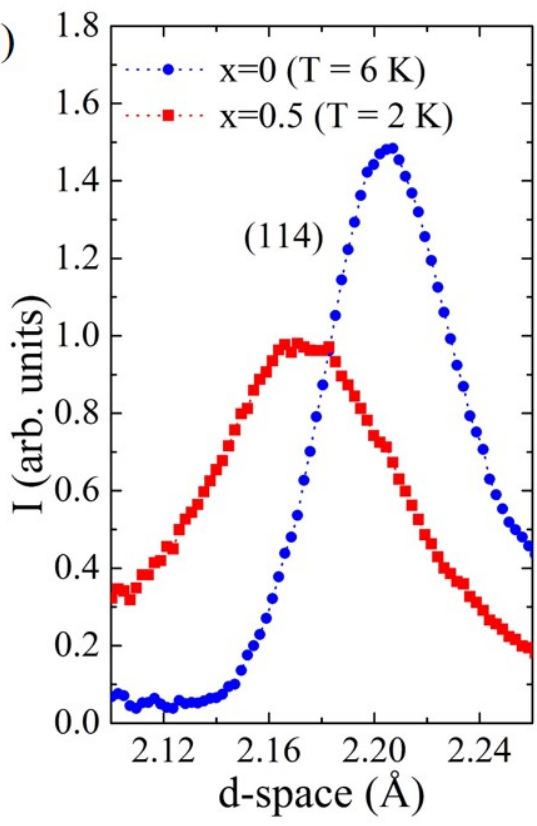

Figure 4.6: The comparison of (a) (110) (b) (114) Bragg peaks between the $x=0$ and $x=0.5$ compositions showing a significant difference of the nonzero $l$ peaks with with $x$.

The structural parameters and goodness of fit obtained from Rietveld refinement are summarized in Table 4.1. $a$ lattice constant gradually increases and the $c$ lattice constant gradually decreases upon F doping as shown in Fig. 4.7. The increase of $a$ is negligibly small $\left(\sim 5 \times 10^{-3} \AA\right)$ compared to the significant decrease of $c(\sim 0.5 \AA)$ at $x=0.5$. The decrease of $c$ lattice constant also had been observed previously with high pressure annealing as well with the simultaneous occurrence of a higher $\mathrm{T}_{c}$ [64]. Therefore, in both doping and high pressure aspects, the shrink along $c$-axis is positively linked with achieving a higher $\mathrm{T}_{c}$. 
TABLE 4.1: A list of the crystal structure parameters of $\mathrm{LaO}_{1-x} \mathrm{~F}_{x} \mathrm{BiS}_{2}$ obtained from the Rietveld refinement of the diffraction data. $R_{w p}$ and $\chi^{2}$ correspond to the weighted $R$ factor and chi-square. The $\mathrm{O} / \mathrm{F}$ site is at $(0,0,0)$.

\begin{tabular}{llllllll}
\hline \hline & $\begin{array}{l}R_{w p} \\
\chi^{2}\end{array}$ & $a(\AA)$ & $c(\AA)$ & $\begin{array}{l}\mathrm{La} \\
2 \mathrm{c} \\
(0.5,0, \mathrm{z})\end{array}$ & $\begin{array}{l}\mathrm{Bi} \\
2 \mathrm{c} \\
(0,0.5, \mathrm{z})\end{array}$ & $\begin{array}{l}\mathrm{S} 1 \\
2 \mathrm{c} \\
(0.5,0, \mathrm{z})\end{array}$ & $\begin{array}{l}\mathrm{S} 2 \\
2 \mathrm{c} \\
(0.5,0, \mathrm{z})\end{array}$ \\
\hline $\mathrm{x}=0$ & 0.0686 & $4.0544(1)$ & $13.8246(8)$ & $0.0904(1)$ & $0.3688(1)$ & $0.3832(4)$ & $0.8096(3)$ \\
$\mathrm{T}=6 \mathrm{~K}$ & 18.84 & & & & \\
\hline $\mathrm{x}=0.2$ & 0.0373 & $4.05618(7)$ & $13.5205(4)$ & $0.09683(9)$ & $0.37514(9)$ & $0.3792(3)$ & $0.8104(2)$ \\
$\mathrm{T}=2 \mathrm{~K}$ & 16.91 & & & & \\
\hline $\mathrm{x}=0.3$ & 0.0417 & $4.05683(7)$ & $13.5083(4)$ & $0.0973(1)$ & $0.37652(9)$ & $0.3805(3)$ & $0.8101(3)$ \\
$\mathrm{T}=2 \mathrm{~K}$ & 16.82 & & & & & \\
\hline $\mathrm{x}=0.5$ & 0.0773 & $4.0596(5)$ & $13.293(4)$ & $0.1035(3)$ & $0.3812(4)$ & $0.3721(8)$ & $0.8181(9)$ \\
$\mathrm{T}=2 \mathrm{~K}$ & 26.21 & & & & & & \\
\hline \hline
\end{tabular}

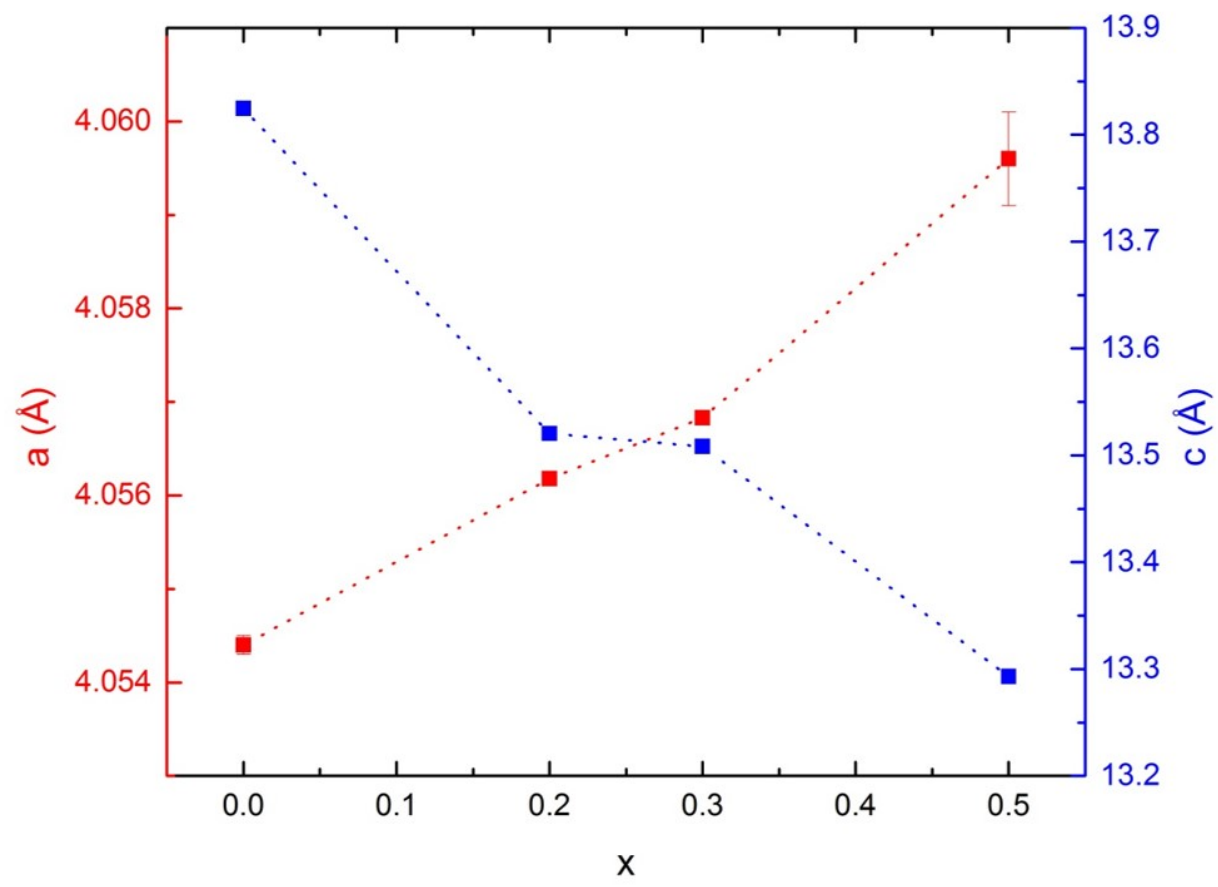

Figure 4.7: The gradual change of lattice constants with F doping.

The tetragonal unit cell consists of an alternating stacking of $\mathrm{La}(\mathrm{O} / \mathrm{F})$ and $\mathrm{BiS}_{2}$ bilayers as seen in Fig. 4.8. The $\mathrm{O} / \mathrm{F}$ atoms occupy the top and bottom atomic layers of the unit cell with four atoms at the corners and one atom at the center of the face of the square and reside at special positions. There are two distinct $\mathrm{S}$ atoms, $\mathrm{S} 1$ and $\mathrm{S} 2$, in the 
unit cell. The Bi ions form a square pyramidal edge shared lattice, coordinated by the four in-plane (S1) and the one out-of-plane (S2) sulfur ions.

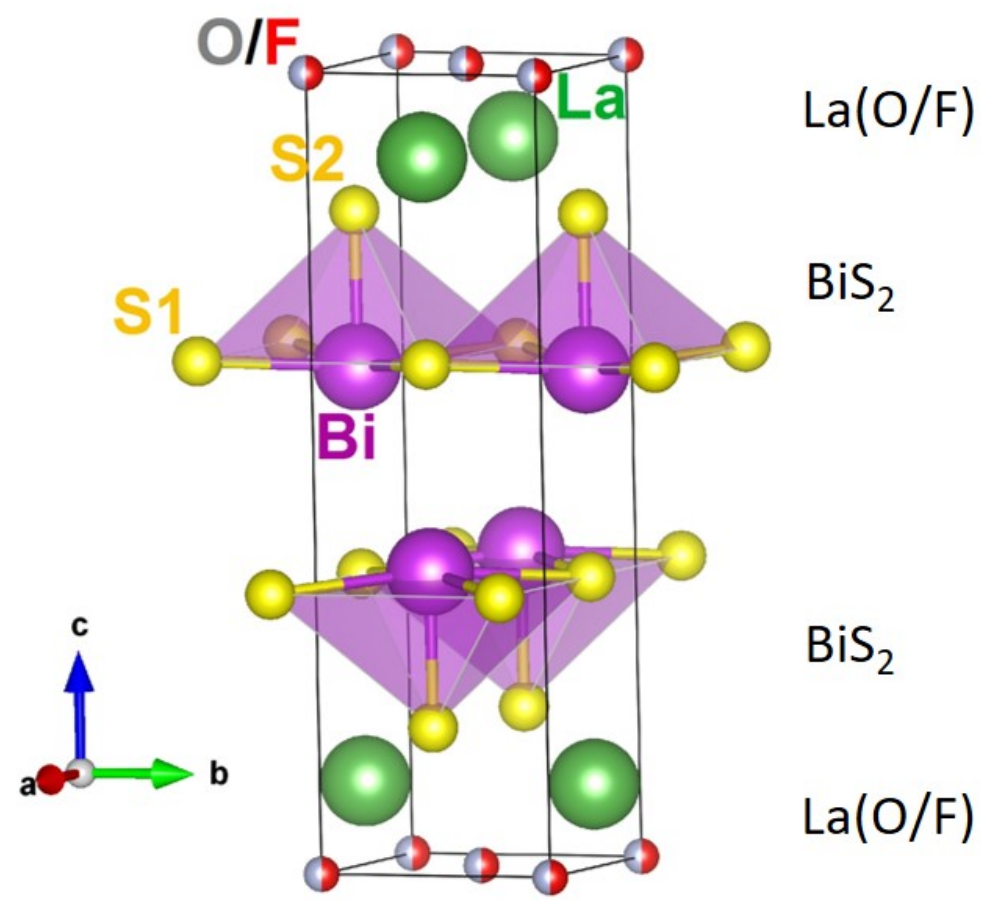

Figure 4.8: The unit cell of $\mathrm{LaO}_{1-x} \mathrm{~F}_{x} \mathrm{BiS}_{2}$ with the BiS tetrahedra.

The change in buckling angle of Bi-S1-Bi as a function of composition is shown in Fig. 4.9(a). The buckling angle of $0^{\circ}$ refers to a flat plane of $\mathrm{BiS}_{2}$ where both $\mathrm{Bi}$ and $\mathrm{S} 1$ have the same position along $c$ direction. It is clear that as a function of composition, the buckling angle of Bi-S1-Bi gradually decreases and becomes negative at $\mathrm{x}=0.5$. Hence the plane gradually unbuckles when $x$ goes from 0 to 0.3 and reverses its orientation at $x=0.5$ such that the $\mathrm{S} 1$ atoms lie closer to the $\mathrm{La}(\mathrm{O} / \mathrm{F})$ spacer layer than the Bi. The buckling of the $\mathrm{BiS}_{2}$ plane was previously reported to be decreasing with the increasing doping level of $\mathrm{F}[112]$ and expected to be flat at $x=0.5$ [15]. However, at 
$x=0.5$, the buckling surpasses the zero line and reverses its orientation contradicting the theoretical models. The change of orientation is depicted in Fig. 4.9(b) for $x=0$ and 0.5 for comparison. In the tetragonal symmetry, the base of the pyramid is a perfect square, and the Bi-S1 bonds are of the same length. The Bi-S1 planes of the parent composition and superconducting $x=0.5$ contain Bi-S1 bonds of $\sim 2.87 \AA$, joined with buckling angles of $172.1^{\circ}$ and $184.8^{\circ}$, respectively. The out-of-plane Bi-S2 bond length is significantly shorter compared to the Bi-S1 bond length in all compositions. Shown in Fig. 4.9(a) is the composition dependence of the Bi-S2 bond length. It is clear that as the system enters the superconducting state, S2 lies further apart from Bi compared to the parent composition increasing the Bi-S2 bond length by $0.18 \AA$. 
(a)

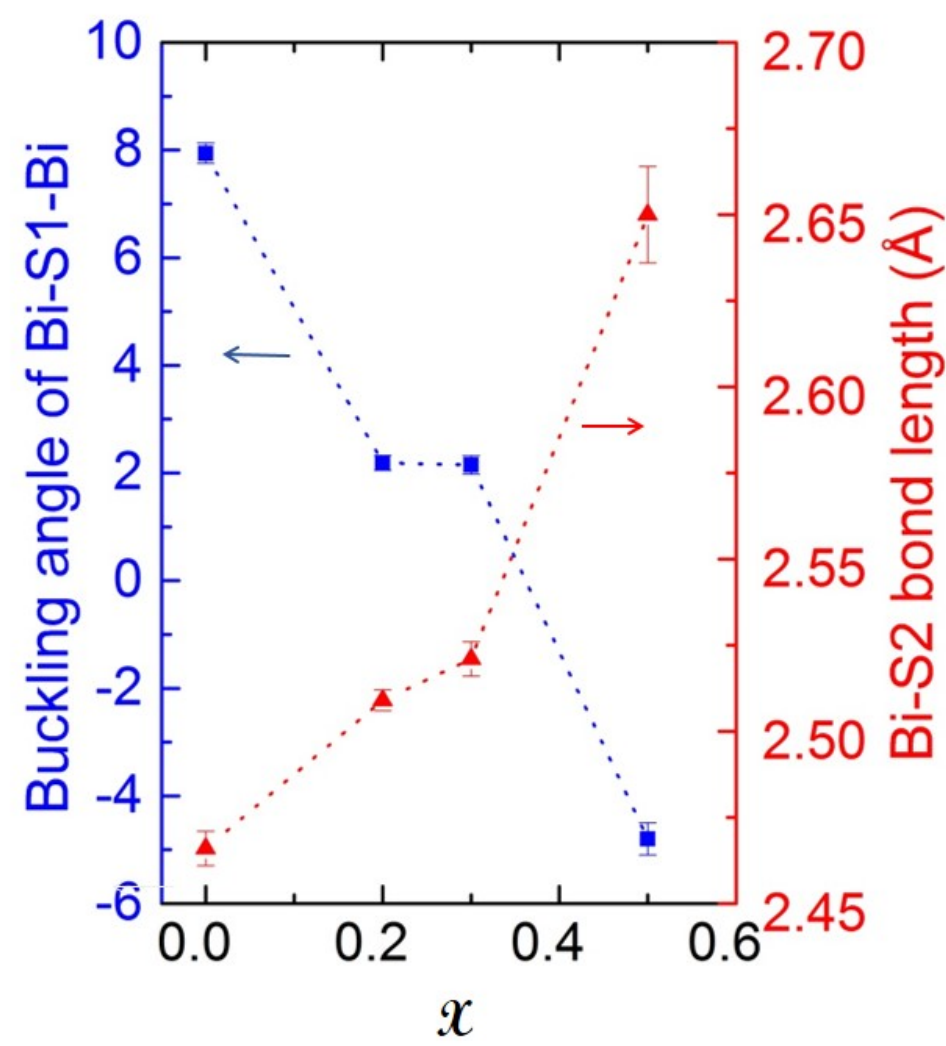

(b)
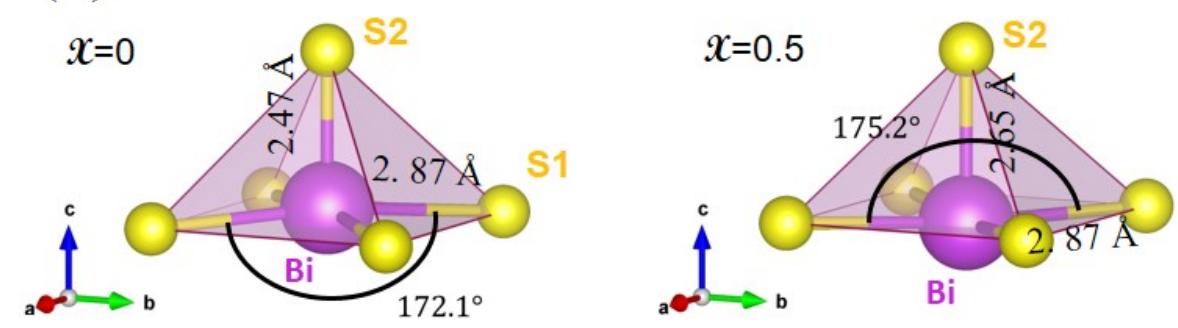

Figure 4.9: The change of buckling angle (in degrees) with respect to a flat $\left(0^{\circ}\right)$ $\mathrm{BiS}_{2}$ plane (blue) and the bond length of Bi-S2 change (red) as a function of doping level. The $\mathrm{BiS}_{2}$ tetrahedron at (b) $x=0$ and (c) $x=0.5$ with a buckling angle of $\sim 172.1^{\circ}$ and a reversed buckling angle of $\sim 175.2^{\circ}$, respectively.

\subsection{The Debye temperature of $\mathrm{LaO}_{1-x} \mathrm{~F}_{x} \mathrm{BiS}_{2}$}

The pair density functions (PDFs) were obtained and analyzed in real space. Shown in the Fig. 4.10 is the temperature dependence of the PDF corresponding to the local structure of $\mathrm{LaOBiS}_{2}$ where the atom-atom labels correspond to the atomic correlations of the specific region of the PDF. The peak broadening due to thermal vibrations is 
uniform except around 2.6 - $3 \AA$ where the Bi-S1 and O-O atomic pairs mostly contribute. The irregularity of the peak broadening in the specific region suggests a certain peculiar temperature dependent motion of corresponding atoms that will be explored later in this dissertation. [95, 115].

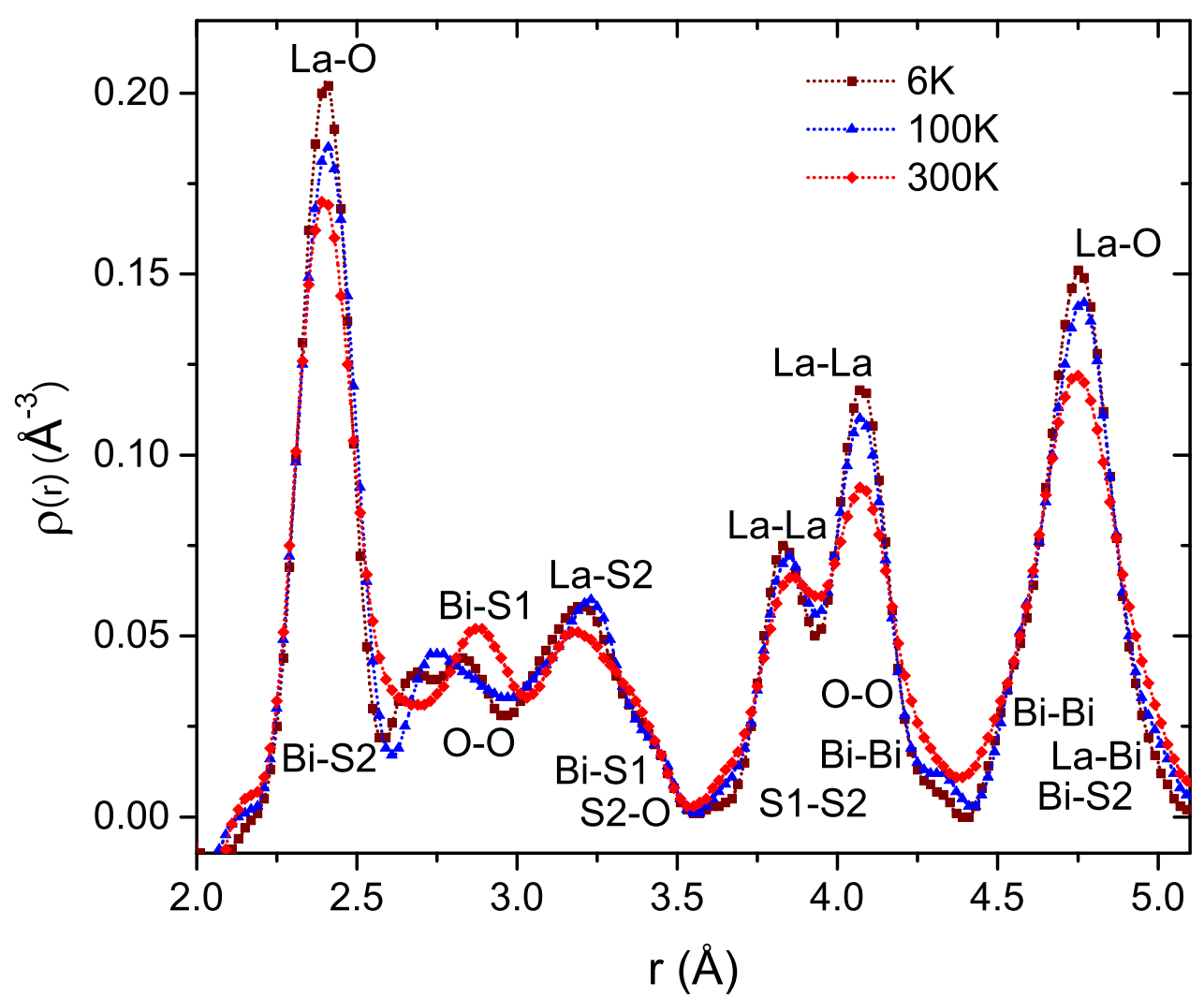

FiguRE 4.10: The temperature dependence of the pair density function of $\mathrm{LaOBiS}_{2}$. $[114]$

The integrated area under the PDF peaks represent the coordination number of atoms in the specified range of the real space where the specific peak lies. The first PDF peak of $\mathrm{LaOBiS}_{2}$ corresponds to both La-O and Bi-S2 atomic correlations as labeled in Fig. 4.10. The FWHM values were extracted from the first PDF peak of $\mathrm{LaOBiS}_{2}$ and $\mathrm{LaO}_{0.5} \mathrm{~F}_{0.5} \mathrm{BiS}_{2}$ and the temperature dependence of the squared $\mathrm{FWHM}$ values is shown in Fig. 4.11. The effects of the lattice vibrations on the PDF peak widths were modelled using a correlated Debye model and compared with the experimentally determined values 
as shown in the figure. The solid line corresponds to the calculated values from the correlated Debye model. The Debye temperature was determined to be 319(29) K and 224(10) $\mathrm{K}$ for $\mathrm{LaOBiS}_{2}$ and $\mathrm{LaO}_{0.5} \mathrm{~F}_{0.5} \mathrm{BiS}_{2}$, respectively. The Debye temperature of $\mathrm{LaO}_{0.5} \mathrm{~F}_{0.5} \mathrm{BiS}_{2}$ found by this method is very close to the previously found value of 221 $\mathrm{K}[26,116]$ from specific heat measurements. The lower $\theta_{D}$ in $\mathrm{LaO}_{0.5} \mathrm{~F}_{0.5} \mathrm{BiS}_{2}$ compared to the parent compound indicates a softer lattice that correlates to the intrinsic disorder evidenced in the diffraction pattern. [114]

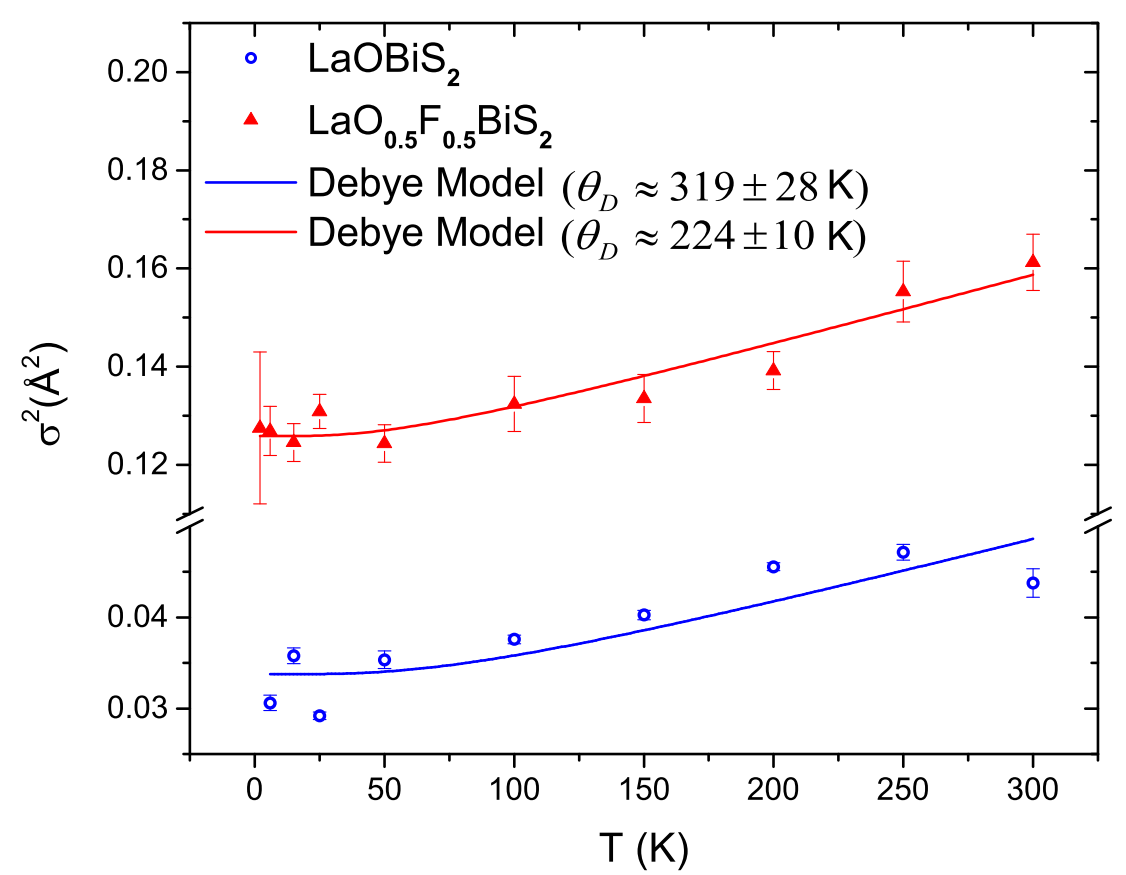

FiguRE 4.11: The temperature dependence of squared FWHM values of the first peak of the local structural data of $\mathrm{LaOBiS}_{2}$, extracted from a Gaussian fitting. The fit of correlated Debye model is represented by the solid line. The error bars were calculated using one standard deviation of FWHM values of the Gaussian fitting. [114] 


\subsection{Local structure of $\mathrm{LaO}_{1-x} \mathrm{~F}_{x} \mathrm{BiS}_{2}$}

\subsubsection{Composition dependence of local structure}

In the diffraction patterns of $\mathrm{LaO}_{1-x} \mathrm{~F}_{x} \mathrm{BiS}_{2}$, broadening of Bragg peaks that go beyond the resolution limit of the instrument was observed at the higher doping levels. Also the diffuse scattering was not taken into account in the Rietveld refinement. The peak broadening and diffuse scattering can happen due to several factors. The major factor is the crystal defects such as stacking faults. Beside the stacking faults, the size mismatch between doped and dopant atoms can contribute to Huang scattering, [117] for instance, while thermal diffuse scattering (TDS) due to phonons and static displacements of ions can additionally contribute to the diffuse scattering, thus decreasing the intensities of the crystalline peaks. However, the atomic sizes of $\mathrm{O}$ and $\mathrm{F}$ are very close so that the peak broadening upon fluorine doping cannot be due to the mismatch of atomic sizes of $\mathrm{O}$ and F. We previously estimated the Debye temperature to be around $200 \mathrm{~K}$ for the $x$ $=0.5$ which indicates that TDS may not be insignificant in the temperatures of interest here which are less than $10 \mathrm{~K}$. To investigate the origin of the Bragg peak broadening, we used PDF analysis to extract the atom specific characteristics of the distortions and their real-space configuration. This PDF analysis is very sensitive to short-range distortions. The PDF, $\rho(r)$ is plotted in Fig. 4.12 for the four compositions, $x=0,0.2$, 0.3 and 0.4. While in reciprocal space Bragg peak broadening is very pronounced as seen in Fig. 4.5, in real space, the PDF correlation peaks change shape with $x$, an unusual effect that indicates a local rearrangement is taking place that may be driven sterically and/or energetically. The crystal symmetry is invariant under doping and the differences observed in the PDFs of $\mathrm{LaO}_{1-x} \mathrm{~F}_{x} \mathrm{BiS}_{2}$ cannot be explained by the substitution of $\mathrm{F}^{-}$ 
for $\mathrm{O}^{2-}$ as the two have very similar neutron scattering lengths and nominal ionic sizes.

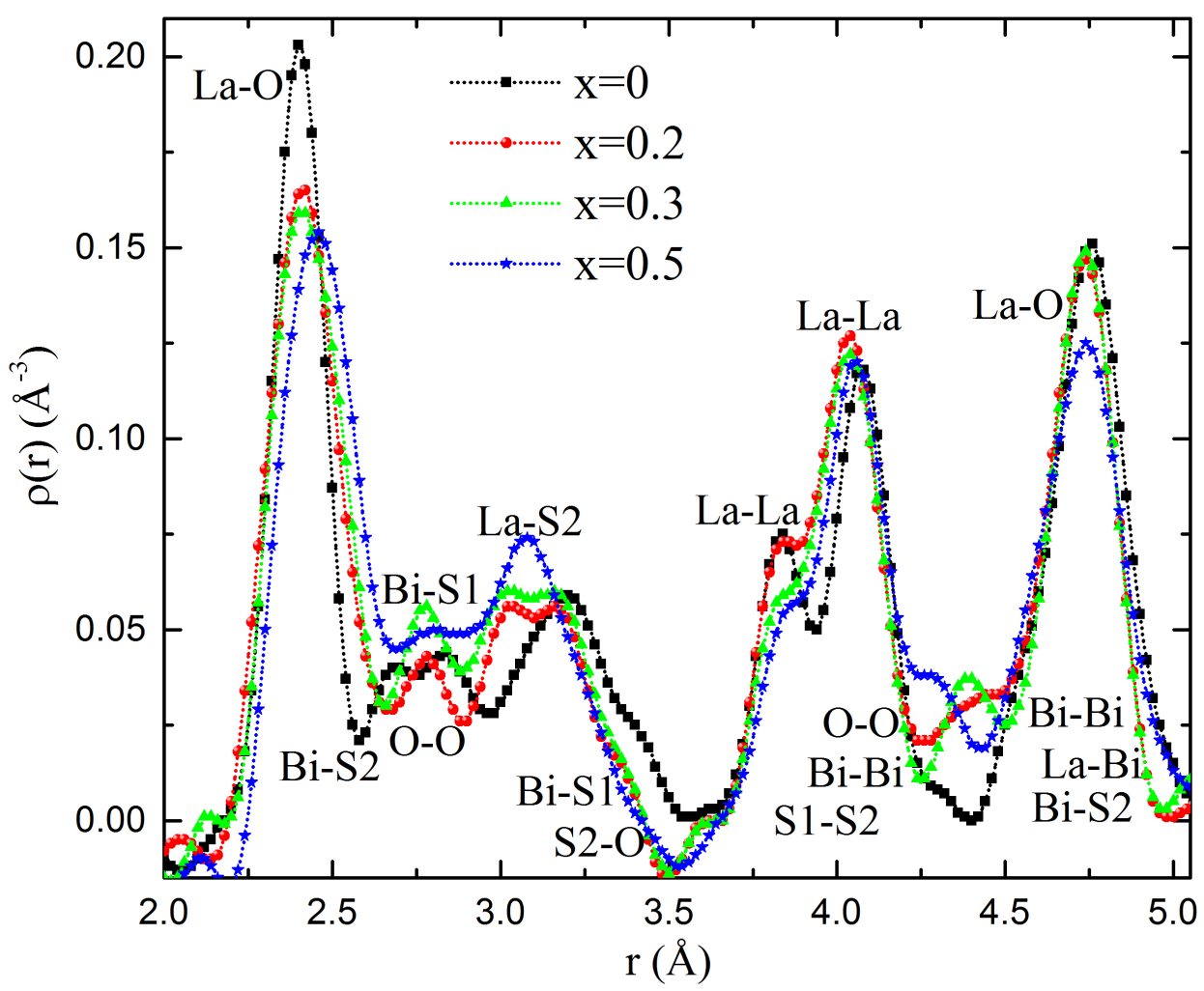

Figure 4.12: Composition dependence of the data corresponding to the local structures of $x=0,0.2,0.3$ and 0.5 . The data of $x=0$ were collected at $6 \mathrm{~K}$ and $x=0.2$, 0.3 and 0.5 were collected at $2 \mathrm{~K}$. 


\subsubsection{Lattice distortions of $\mathrm{LaOBiS}_{2}$}

As a starting point, the PDF data is compared to a model $\rho(r)_{\text {mod }}$, calculated using the unit cell dimensions and atomic coordinates of the average structure obtained by the Rietveld refinement. In Fig. 4.13, $\rho(r)_{\exp }$ (symbols) is compared to the model $\rho(r)_{\text {mod }}$ (average) represented in a dashed blue line. The overall agreement between the two curves is good, however, very clear differences are observed especially around 2.5 -3.5 $\AA$ that are not due to systematic or statistical errors. Some termination ripples can be seen around the first PDF peak resulting from the truncation of the Fourier transform at a finite momentum transfer. However, the differences between the data and the average model are well above the error. The objective is to investigate the nature of local lattice distortions that gives rise to these differences that cannot be explained by the crystal/average structure that represents the long range order. 


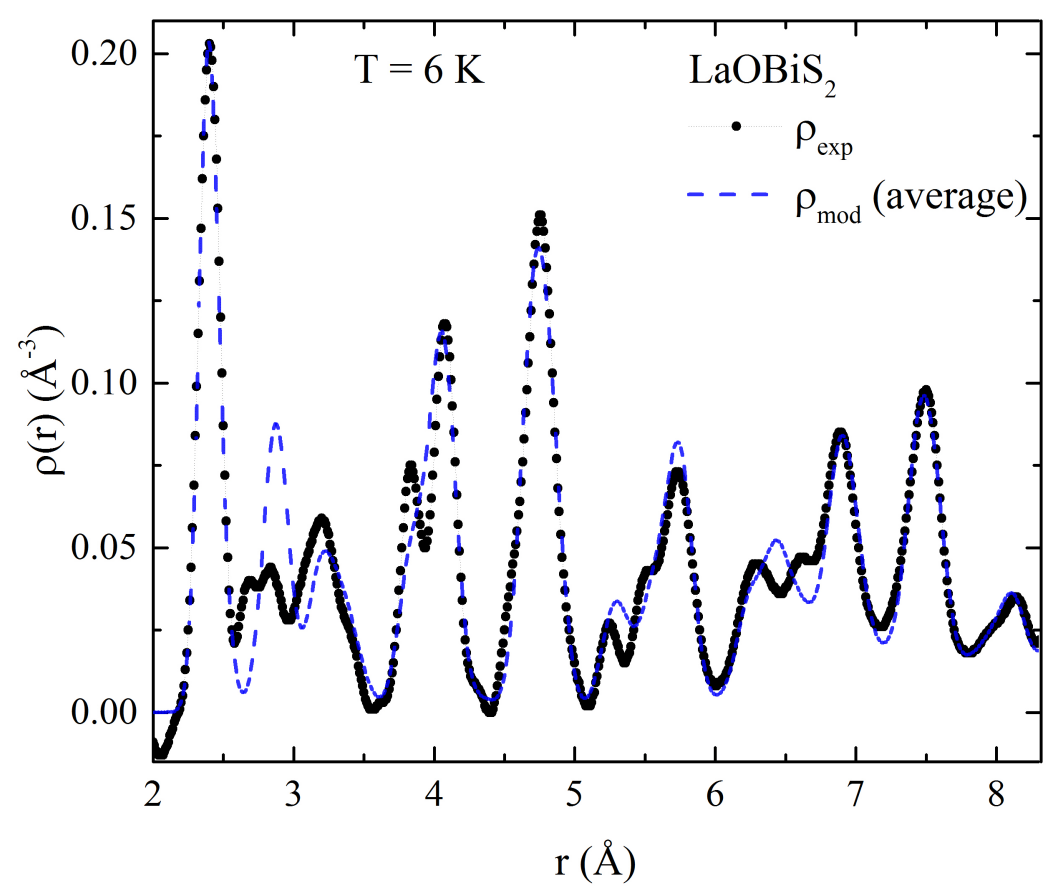

Figure 4.13: The PDF of $\mathrm{LaOBiS}_{2}$ at $6 \mathrm{~K}$ (black symbols) is compared to the PDF of average model (dashed blue line).

\subsubsection{Nature of lattice distortions in $\mathrm{LaOBiS}_{2}$}

In-plane S1 displacements can reproduce the local structure of $\mathrm{LaOBiS}_{2}$ with two types of displacement configurations as shown in Fig. 4.14. In Fig. 4.14(a), the displacement mode is of the antiferrodistortive type and symmetric around Bi atom. It creates short and long Bi-S1 bonds that affect the charge distribution around $\mathrm{Bi}$ and thus creates a double-well displacement potential of the Bi-S1 bonds. The data can also be reproduced equally well using the ferrodistortive type displacement mode shown in Fig. 4.14(b). In this model, the S1 atoms are displaced in a ferrodistortive type mode, and create short and long Bi-S1 bonds in an asymmetric way. 


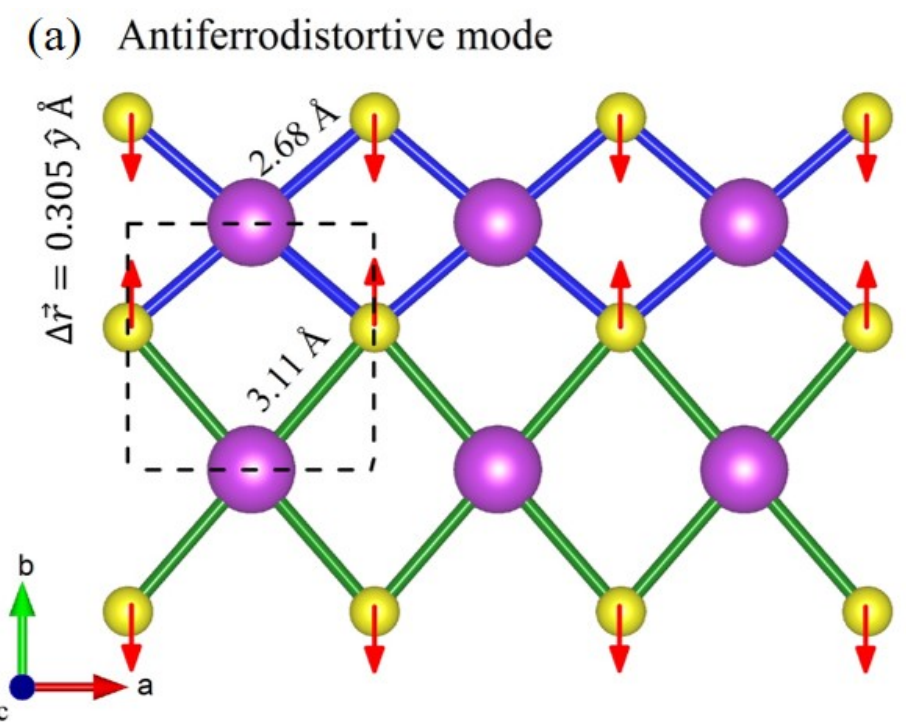

(b) Ferrodistortive mode

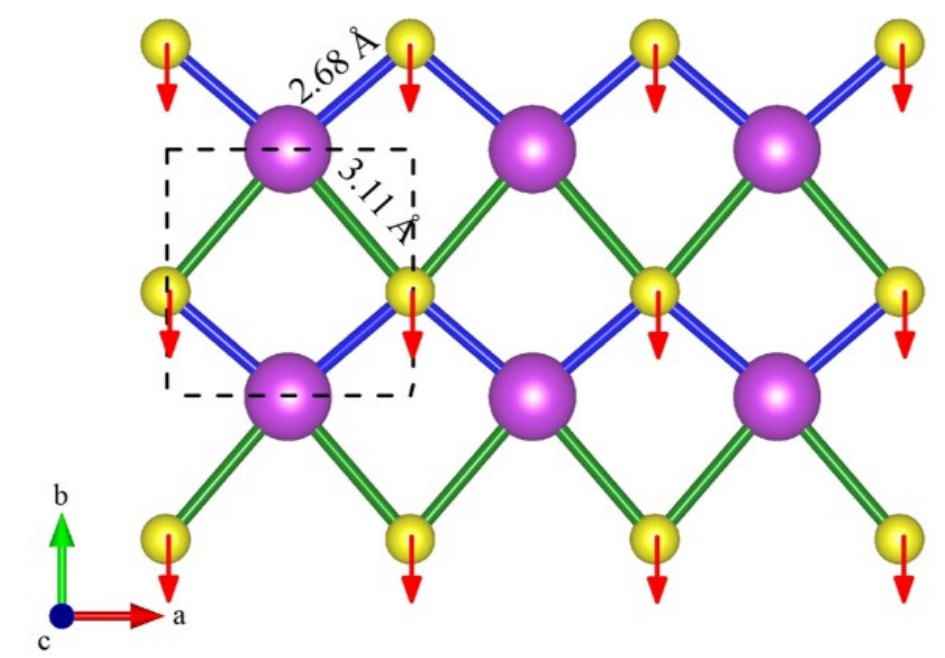

Figure 4.14: (a) A schematic of the $\mathrm{BiS}_{2}$ plane showing the antiferrodistortive type mode. (b) A schematic of the $\mathrm{BiS}_{2}$ plane showing the ferrodistortive type mode. The displacements of $\mathrm{S} 1$ are either in the $\mathrm{x}$ or y direction.

Each of the mode, antiferrodistortive or ferro-distortive, can further be categorized into several types depending on the different displacement directions of S1 in the two BiS1 planes (at $\mathrm{z} \sim \frac{1}{3}$ and $\mathrm{z} \sim \frac{2}{3}$ ) indicated in Fig. 4.15. Given the tetragonal symmetry, all the direction dependent variations of the displacements are equivalent to three main types. In $(\mathrm{x},-\mathrm{y})$ type, the $\mathrm{S} 1$ in plane 1 is displaced along $\mathrm{x}$ direction and in plane 2 , it is displaced along -y direction. All $(\mathrm{x}, \mathrm{y}),(-\mathrm{x}, \mathrm{y}),(\mathrm{y}, \mathrm{x}),(-\mathrm{x},-\mathrm{y}),(-\mathrm{y}, \mathrm{x}),(\mathrm{y},-\mathrm{x})$ and 
$(-\mathrm{y},-\mathrm{x})$ modes are equivalent to $(\mathrm{x},-\mathrm{y})$ type by symmetry. The other two types of modes are $(\mathrm{x}, \mathrm{x})$ and $(\mathrm{x},-\mathrm{x})$. All these modes yield the same local bond order but might be different energetically. Note that within one unit cell, the two $\mathrm{BiS}_{2}$ planes are rotated by 90 degrees. The $(\mathrm{x},-\mathrm{x})$ breaks the 4 -fold rotational symmetry to a 2 -fold while the inversion symmetry remains. The $(\mathrm{x},-\mathrm{y})$ distortions break the 4 -fold and 2 -fold rotational as well as the inversion symmetries.

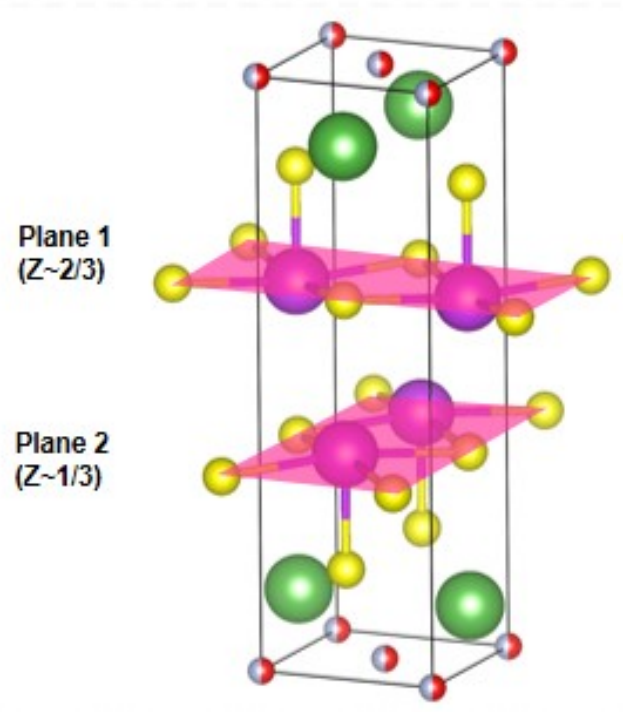

Figure 4.15: The two Bi-S1 planes in one unit cell of $\mathrm{LaO}_{1-x} \mathrm{~F}_{x} \mathrm{BiS}_{2}$ at $\mathrm{z} \sim 1 / 3$ and $\mathrm{z} \sim 2 / 3$.

The long and short Bi-S1 in-plane bonds of antiferrodistortive mode indicate a variance in the $\mathrm{Bi}$ valence due to charge disproportionation. The proposed charge fluctuations are shown in the schematic of Fig. 4.16 for the BiS planes for the three types of antiferrodistortive modes. In fact, $\mathrm{Bi}$ is already well known for fluctuations of valence and charge disproportion[118, 119].

Shown in the Fig. 4.17 is the new local model of $\rho(r)_{\bmod }$ calculated using the antiferrodistortive mode model (solid red line) compared to the $\rho(r)_{\text {exp }}$. Only S1 is displaced 

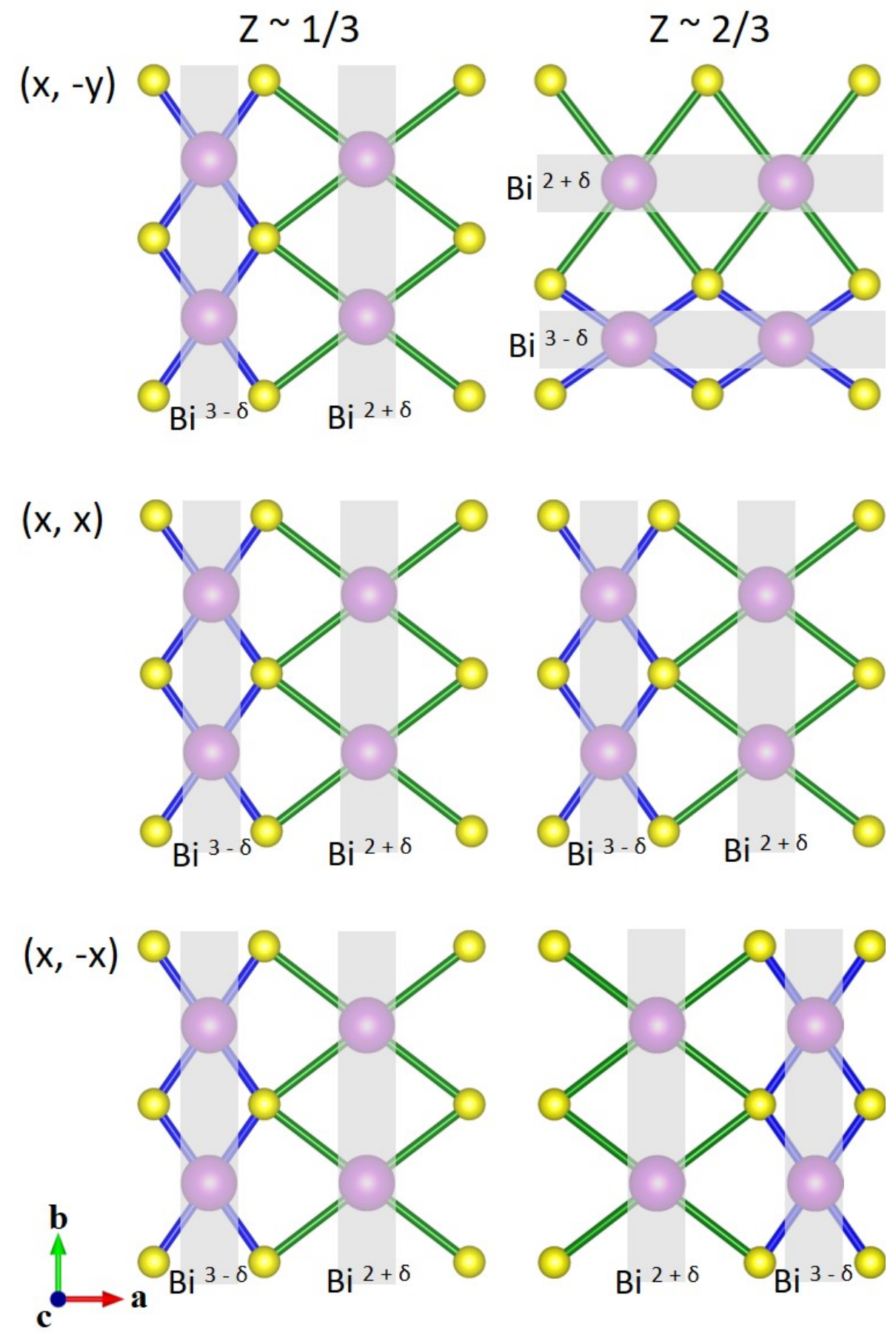

FiguRe 4.16: Stripes of charge fluctuations in the two BiS1 planes of the crystal structure. The $(\mathrm{x},-\mathrm{y}),(\mathrm{x}, \mathrm{x})$ and $(\mathrm{x},-\mathrm{x})$ refer to the $\mathrm{S} 1$ displacement directional coordinates in the $\mathrm{z} \sim 1 / 3$ and $\mathrm{z} \sim 2 / 3$ planes.

following either the antiferrodistortive type or ferrodistortive type, while all other atom coordinates are kept the same as in the average structure. In spite of its simplicity, 
this model fits the data quite well, especially in the 2.5 to $3.5 \AA$ range. Both antiferrodistortive and ferro-distortive modes reproduce the experimental data equally likely as they give rise to identical magnitudes of bonds irrespective of their configuration in real space. It is possible that both the ferrodistortive and antiferrodistortive type modes are locally present.

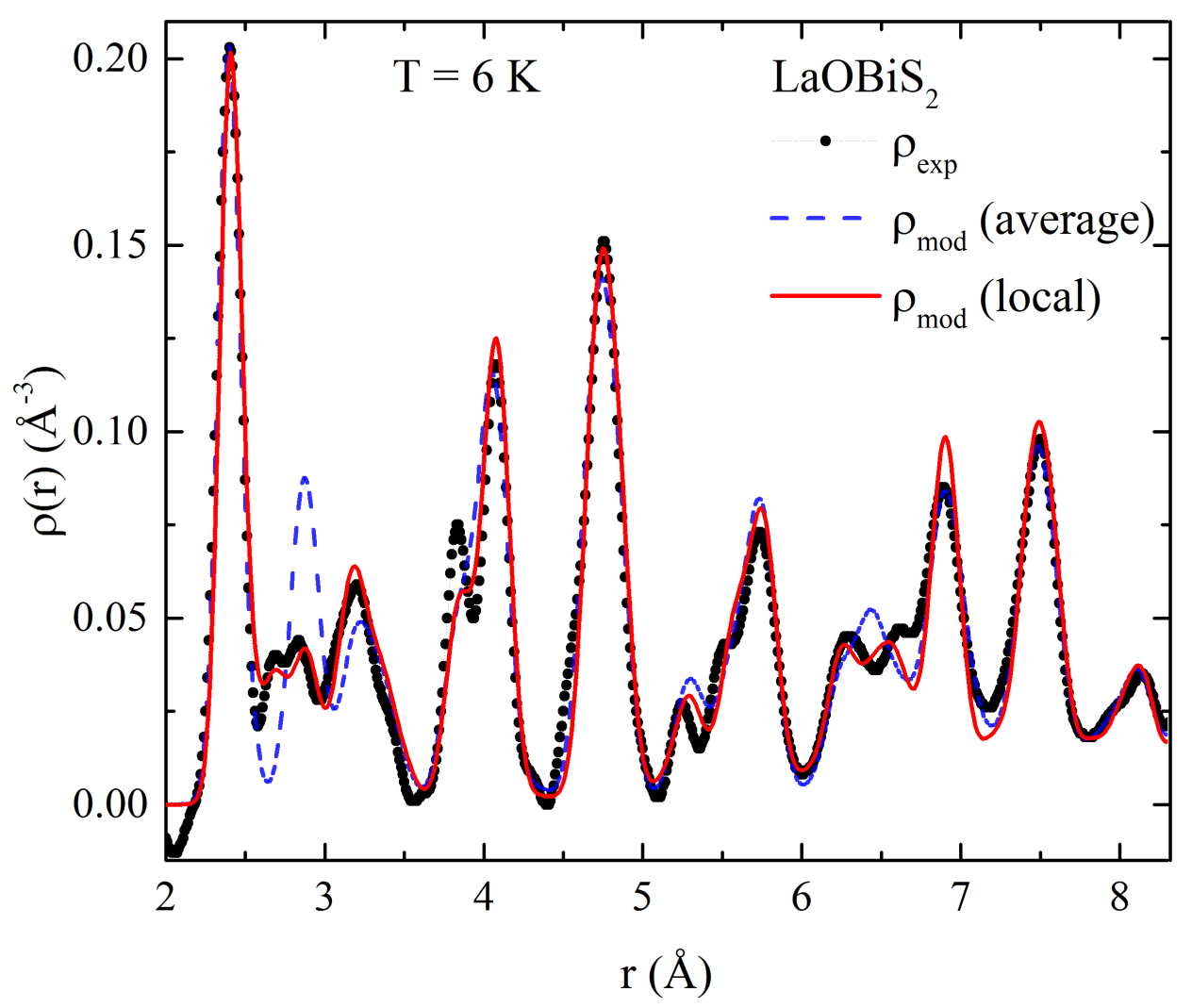

Figure 4.17: The PDF of $\mathrm{LaOBiS}_{2}$ at $6 \mathrm{~K}$ (black symbols) is compared to the PDF of average model (dashed blue line) and the local model (solid red line).

\subsubsection{Lattice distortions in $\mathrm{F}$ doped $\mathrm{LaOBiS}_{2}$}

In all the $\mathrm{F}$ doped samples of $\mathrm{LaOBiS}_{2}$, similar in-plane sulfur distortions are present. The $\rho(r)_{\exp }$ of $\mathrm{LaO}_{1-x} \mathrm{~F}_{x} \mathrm{BiS}_{2}$ at $x=0.5$ is shown in Fig. 4.18 and is compared to the 
$\rho(r)_{\text {mod }}$ for the average model of $x=0.5$ represented by the dash blue line. Differences are observed between the two in the same region of real space as in the parent compound even though the shape of the PDF peaks are different. Just as in the parent compound, the antiferrodistortive and ferrodistortive modes can fit the data quite well $\left(\rho(r)_{m o d}\right.$ is shown in a solid red line) however, the magnitude of distortion is different.

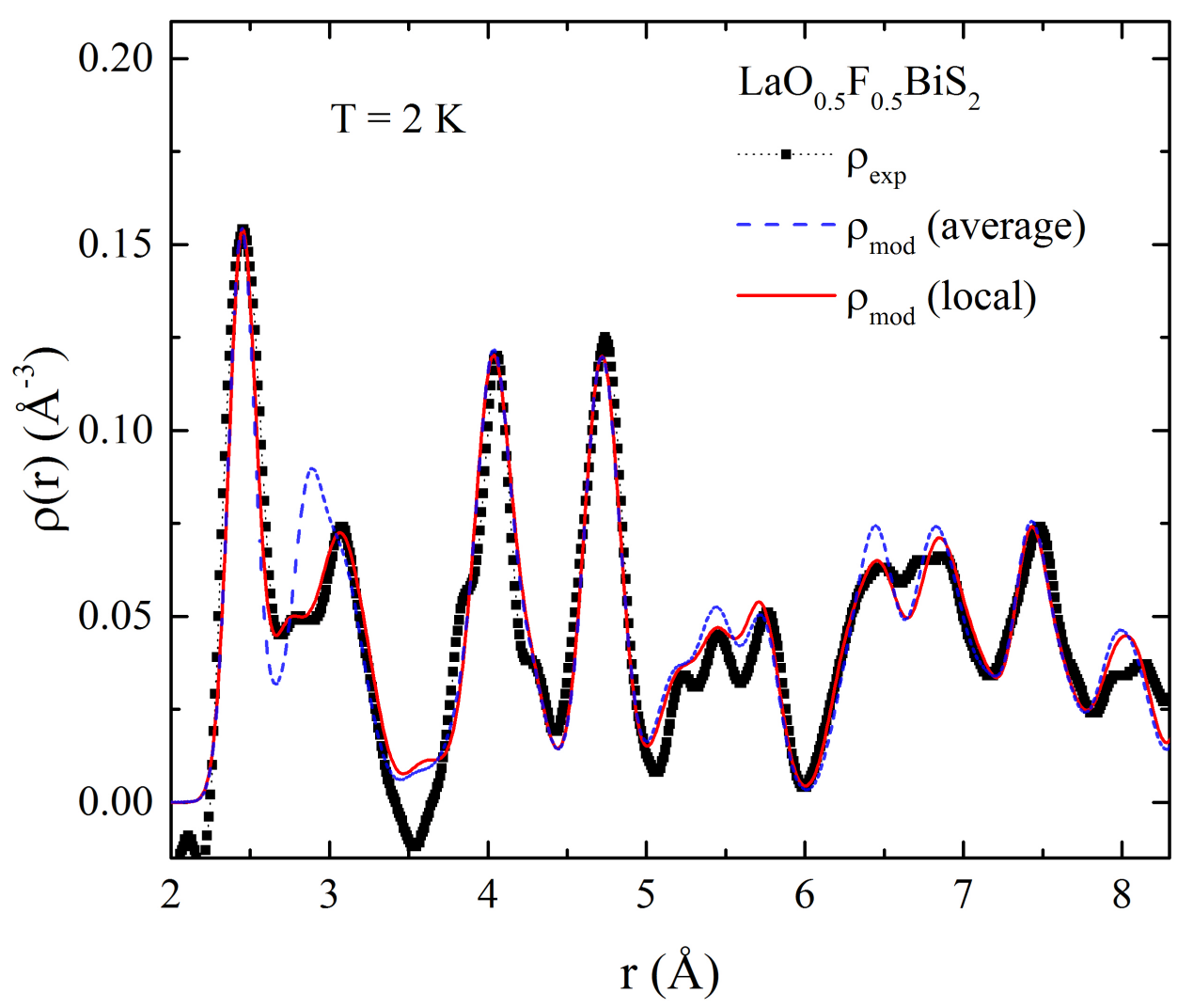

Figure 4.18: The PDF of $\mathrm{LaOBiS}_{2}$ at $6 \mathrm{~K}$ (black symbols) is compared to the PDF of average model (dashed blue line) and the local model (solid red line).

The PDF is a superposition of partial PDFs corresponding to all correlations in the sample. A partial PDF of one correlation represents the probability distribution of finding different magnitudes of bond lengths between the corresponding pair of atoms in the real space. Shown in Fig. 4.19 are the Bi-S1 and Bi-S2 partial PDFs obtained from the local and average models for $x=0$ and $x=0.5$. It can clearly be seen that the 
Bi-S1 bond of average models at $2.8 \AA$ is split to two in the local models in both the $x$ $=0$ and 0.5 , while the split is larger in the $x=0$ than in $x=0.5$ sample. A third small peak is also observed in close proximity to this bond that is from Bi-S1 bond lengths across planes. The bond lengths of the local structure for all the samples $x=0,0.2,0.3$ and 0.5 are summarized in Table 2 .

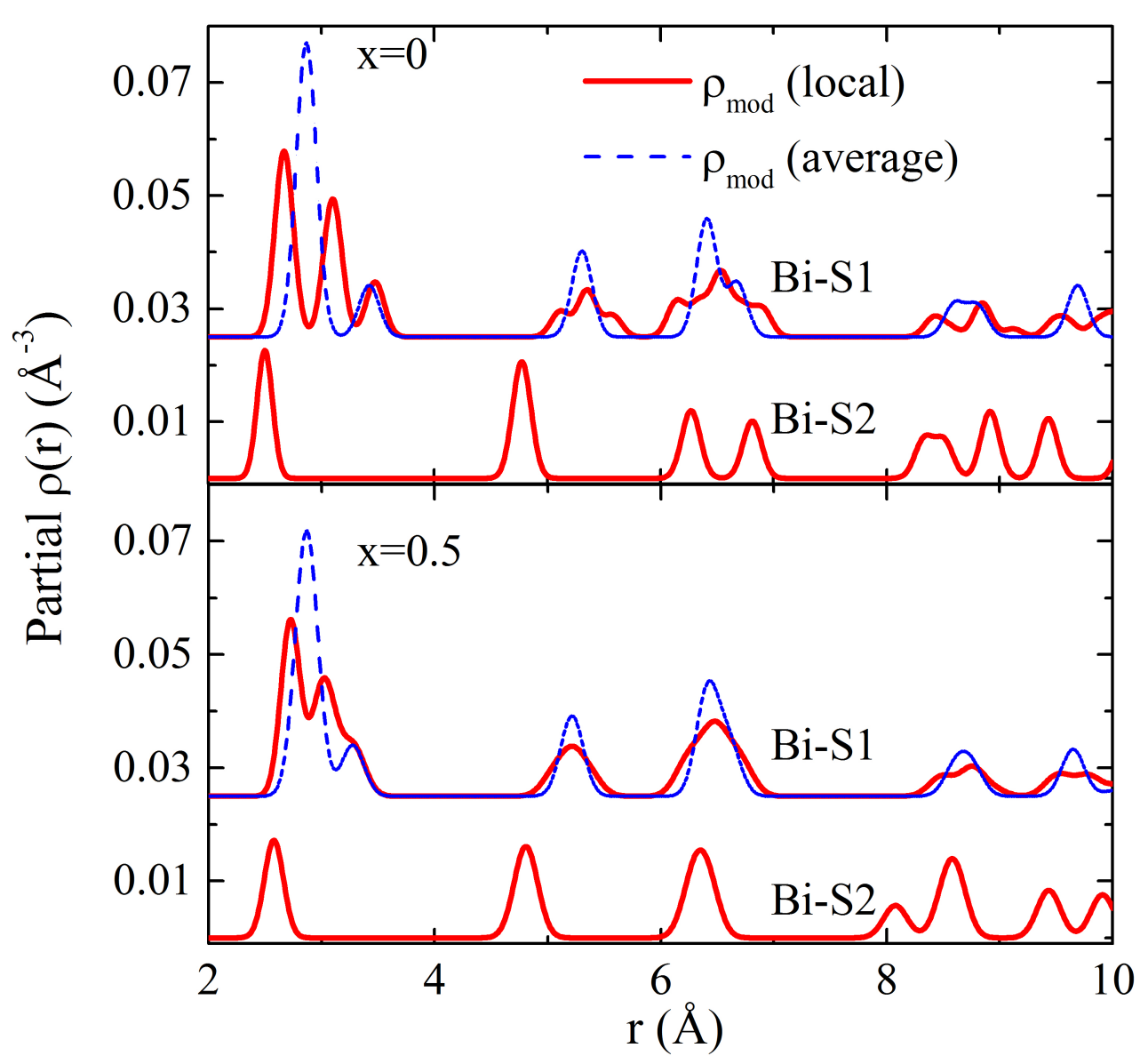

Figure 4.19: The partial PDFs of Bi-S1 and Bi-S2 for $x=0$ (top) and $x=0.5$ (bottom).

The charge fluctuation inferred from the long and short Bi-S1 bond lengths is present in all compositions leading to the $x=0.50$ which is the only superconducting composition in our series. With doping, the Bi - S1 bonds differ in length as shown in the schematic of 
TABLE 4.2: A list of Bi-S1 bond lengths in the local structure.

\begin{tabular}{lccl}
\hline & \multicolumn{2}{c}{ Bi-S2 bond length $(\AA)$} & \\
\cline { 2 - 3 } & Short & Long & Bi-S2 bond length $(\AA)$ \\
\hline$x=0, \quad \mathrm{~T}=6 \mathrm{~K}$ & 2.68 & 3.11 & 2.5 \\
$x=0.2, \mathrm{~T}=2 \mathrm{~K}$ & 2.72 & 3.03 & 2.51 \\
$x=0.3, \mathrm{~T}=2 \mathrm{~K}$ & 2.74 & 3.01 & 2.52 \\
$x=0.5, \mathrm{~T}=2 \mathrm{~K}$ & 2.73 & 3.03 & 2.58 \\
\hline
\end{tabular}

the Bi tetrahedra of Fig. 4.20. We can also see that upon entering the superconducting phase, the apical S2 atom gets further away from the planes and closer to the $\mathrm{La}(\mathrm{O} / \mathrm{F})$ charge layers. The S2 atom can mediate the transfer of charge from the doping layers to the conduction $\mathrm{BiS}_{2}$ layers just like the chain oxygen does in $\mathrm{YBa}_{2} \mathrm{Cu}_{3} \mathrm{O}_{7-\delta}$.[120]
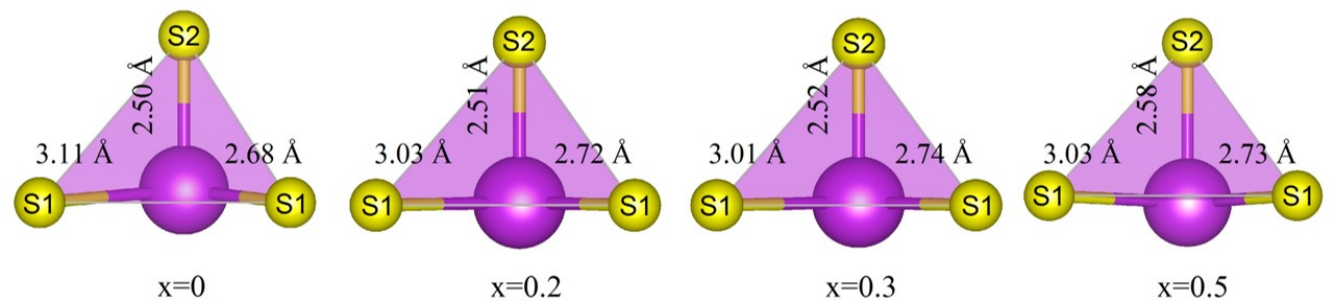

FiguRE 4.20: A schematic of the Bi-S tetrahedron with the bond lengths detremined from the refinement of the local structures of $x=0,0.2,0.3$ and 0.5 .

\subsubsection{Temperature dependence of bond length splitting}

In both the non superconducting $x=0$ and superconducting $x=0.5$, the in-plane Bi-S1 bond length split as a result of S1 displacement. However, the local S2 displacement along the $c$-axis is present only in the superconducting $x=0.5$. With temperature, the split of the Bi-S1 bonds in the $x=0$ remains almost same in the entire temperature range from $2 \mathrm{~K}$ to $300 \mathrm{~K}$ as shown in Fig. 4.21. In the $x=0.5$, the split of the Bi-S1 bonds changes only little with decreasing temperature, however, on approaching $\mathrm{T}_{c}$, the split becomes larger as shown in Fig. 4.22. On the other hand, in $x=0.5$, it is clear that with cooling, the height of the S2 atom gets closer to the donor planes as shown in Fig. 
4.22(b). This trend is also consistent with the temperature dependence of S2 in the average structure although the bond length values are different.

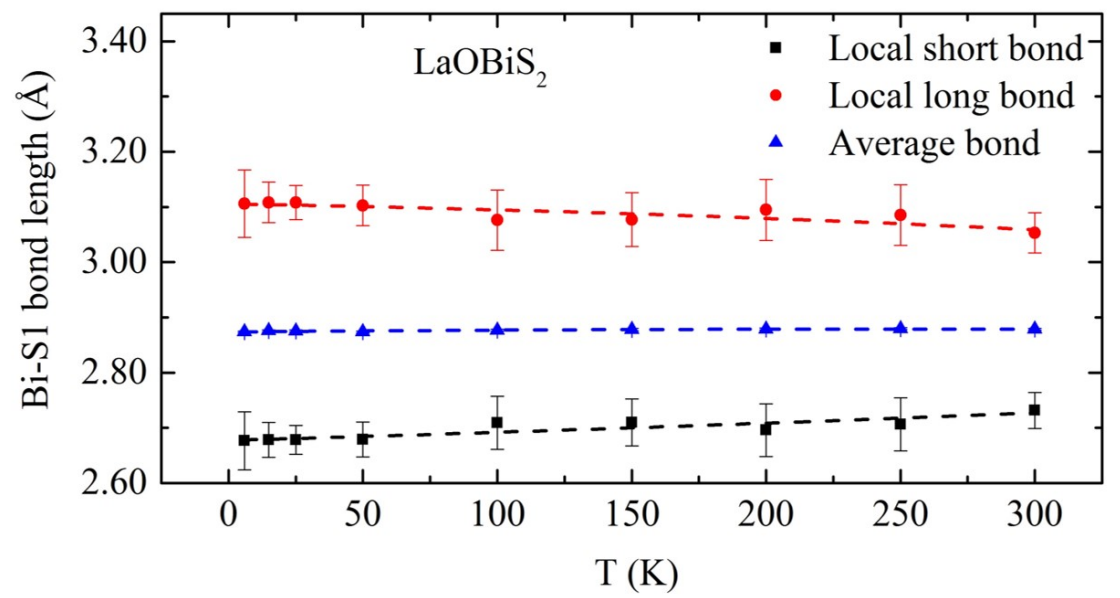

FiguRE 4.21: The temperature dependence the short (square) and long (circle) local $\mathrm{Bi}-\mathrm{S} 1$ bond lengths is contrasted to the Bi-S1 average bond length (triangle) in $\mathrm{LaOBiS}_{2}$. 


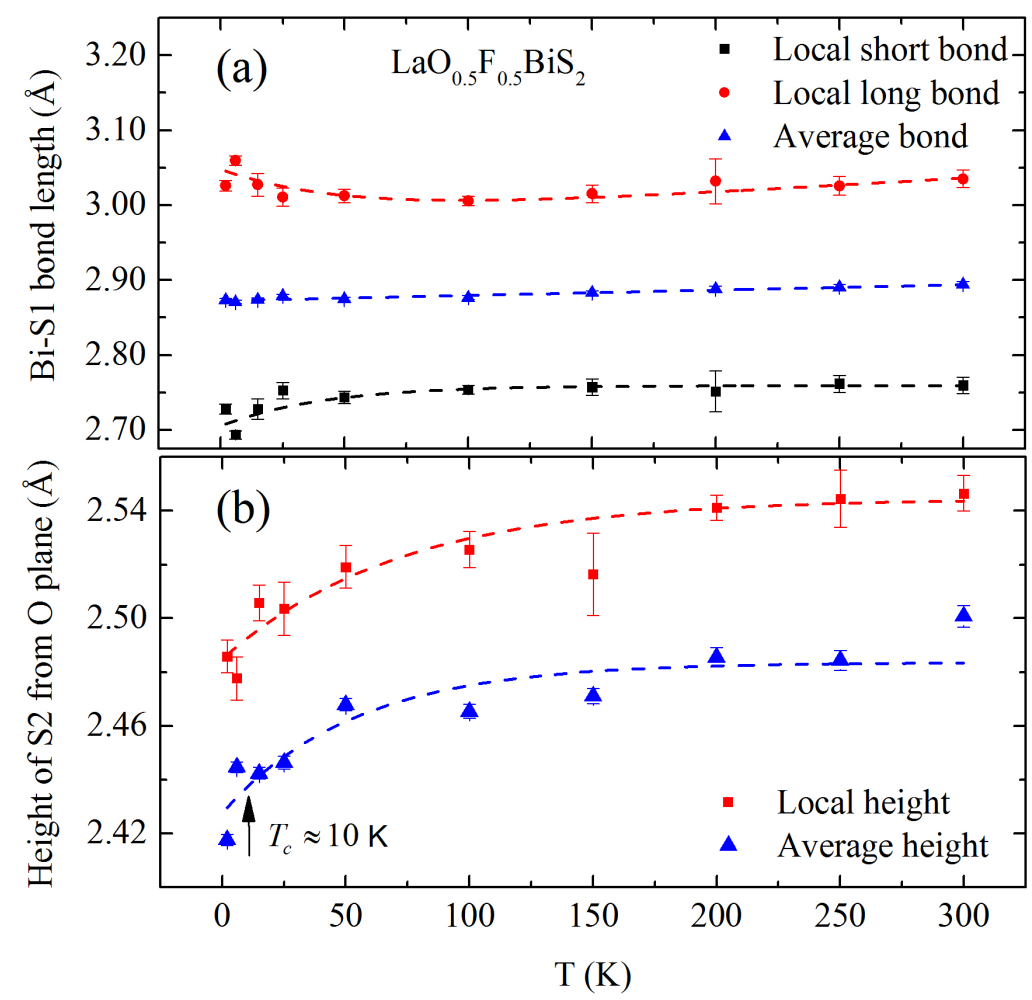

Figure 4.22: The temperature dependence of bond lengths in $\mathrm{LaO}_{1-x} \mathrm{~F}_{x} \mathrm{BiS}_{2}$. (a) The short (square) and long (circle) local Bi-S1 bond lengths is contrasted to the Bi-S1 average bond length (triangle). (b) The height of the S2 atom from the oxygen plane obtained from fitting the data of the local structure and from the Rietveld refinement of the average structure. [127]

\subsection{Summary}

A long-range order exists in all the samples as evidenced from the Bragg structure. The $\mathrm{BiS}_{2}$ planes are buckled in the non-superconducting and superconducting samples, however, the buckling decreases with $\mathrm{F}$ doping and again increases in the opposite orientation in the superconducting phase at $x=0.5$. On the other hand, in the superconducting sample, the disorder is strong not only due to the distortions of the $\mathrm{BiS}_{2}$ planes but also due to the c-axis strain. As inferred by the extracted Debye temperature, the superconducting $x=0.5$ has a softer lattice than its parent sample, thus implying enhanced phonons. 
Superconductivity in $\mathrm{LaO}_{1-x} \mathrm{~F}_{x} \mathrm{BiS}_{2}$ is strongly related with structural disorder and the average structure is not sufficient to explain the crystal state. The pair density function analysis is sensitive to subtle atomic deviations and reveals that S1 and S2 distortions are important in explaining the lattice instability of the system. The leading mode of local distortions involves sulfur movements in the superconducting plane giving rise to two different Bi-S1 plaquettes. Several possible sulfur displacement modes are present. The stripes of distortions proposed are all short-range and the presence of stacking faults breaks the periodicity thus preventing any long-range order. It is not possible to distinguish whether this is the result of a antiferrodistortive or a ferrodistortive type mode because they yield the same bond magnitude. In the superconducting system, the leading instability mode consists of displacements of S1 in the superconducting plane and out-of-plane S2 distortions. However, the distortions get smaller in the high pressure annealed $x=0.5$ superconducting samples while the out-of plane z-motion of S2 increases. Both ferrodistortive and antiferrodistortive modes create unequal charge distributions. However, such charge fluctuations are local and cannot produce a charge density wave. The split of bond lengths remains almost at a constant value in both superconducting and non-superconducting samples with temperature, however, it becomes larger in superconducting $x=0.5$ when the $\mathrm{T}_{c}$ is approached. 


\section{Chapter 5}

\section{Crystal structure and local lattice distortions in $\mathrm{NdO}_{1-x} \mathbf{F}_{x} \mathrm{BiS}_{2}$ superconductor}

\subsection{Introduction}

A chemical pressure is applied by doping or substituting a site with an isovalent element. In $\mathrm{REO}_{1-x} \mathrm{~F}_{x} \mathrm{BiS}_{2}$ superconductors, the chemical pressure effect on superconductivity has been investigated by either substituting $\mathrm{RE}$ site with isovalent ions as $\mathrm{La}^{3+}, \mathrm{Ce}^{3+}$, $\mathrm{Nd}^{3+}, \mathrm{Pr}^{3+}, \mathrm{Yb}^{3+}$ and $\mathrm{Sm}^{3+}$ and their combinations, e.g. $\mathrm{Nd}_{0.8} \mathrm{Sm}_{0.2}$, or substitution of $\mathrm{S}^{2-}$ by $\mathrm{Se}^{2-}$. The smaller ionic sizes are expected to impose a negative chemical pressure on the sample in a way the pressure is applied inward the unit cell. $\mathrm{NdO}_{1-x} \mathrm{~F}_{x} \mathrm{BiS}_{2}$ exhibits a maximum $\mathrm{T}_{c}$ of $\sim 5 \mathrm{~K}$ at $x \sim 0.4$. [121] which is the highest transition temperature of a $\mathrm{BiS}_{2}$ superconductor with single element at RE site under ambient pressure synthesis conditions. [55, 122]

Shown in Fig. 5.1 is the effect of in-plane chemical pressure for $\mathrm{ReO}_{1-x} \mathrm{~F}_{x} \mathrm{BiS}_{2}$ and $\mathrm{LaO}_{1-x} \mathrm{~F}_{x} \mathrm{Bi}\left(\mathrm{S}_{1-y} \mathrm{Se}_{y}\right)_{2} \cdot[123]$ The in-plane pressure here is defined as, $\frac{R_{B i}+R_{C h}}{B i-C h}$ where 
$R_{B i}$ and $R_{C h}$ are ionic radius of $\mathrm{Bi}$ and Chalcogen ( $\mathrm{S}$ or $\mathrm{Se}$ ), respectively. The Bi-Ch represents the in-plane bond length between $\mathrm{Bi}$ and the Chalcogen. The maximum $\mathrm{T}_{c}$ is obtained with $\mathrm{RE}=\mathrm{Nd}_{0.6} \mathrm{Sm}_{0.4}$. The $\mathrm{RE}=\mathrm{Nd}_{0.6} \mathrm{Sm}_{0.4}$ exceeding the $\mathrm{T}_{c}$ of $\mathrm{RE}=\mathrm{Nd}$ can be explained by the doping with a smaller ion. However, surprisingly, $\mathrm{SmO}_{1-x} \mathrm{~F}_{x} \mathrm{BiS}_{2}$ is not superconducting and $\mathrm{YbO}_{1-x} \mathrm{~F}_{x} \mathrm{BiS}_{2}$ does not show bulk superconductivity although they have smaller ionic radii. [59, 124] Furthermore, it had been observed that the superconductivity of $\mathrm{RE}=\mathrm{La}$ is suppressed by partial Ce doping, and the reason behind it not explicitly reported and attributed to a structural fluctuation.[55]

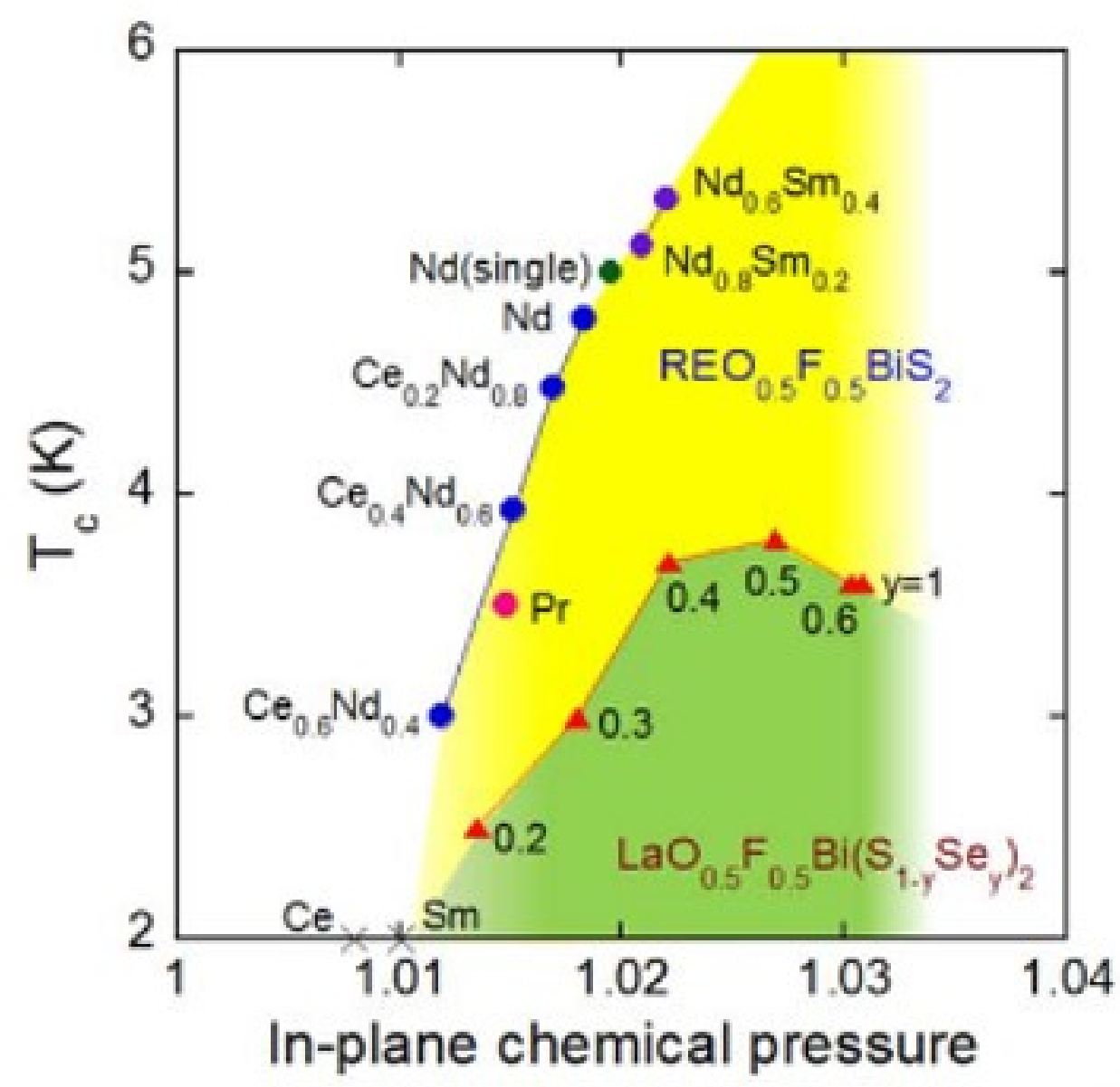

Figure 5.1: In-plane chemical pressure dependence of $\mathrm{T}_{c}$ for $\mathrm{ReO}_{1-x} \mathrm{~F}_{x} \mathrm{BiS}_{2}$ and $\mathrm{LaO}_{1-x} \mathrm{~F}_{x} \mathrm{Bi}\left(\mathrm{S}_{1-y} \mathrm{Se}_{y}\right)_{2} \cdot[123]$ 
$\mathrm{NdO}_{1-x} \mathrm{~F}_{x} \mathrm{BiS}_{2}$, also shows a two superconducting gap feature. The smaller gap correspond to a reduced gap, $2 \Delta / k_{B} \mathrm{~T}_{c}$, of 16.8 which exceeds the BCS theoretical value in the weak coupling limit significantly while the other correspond to a value of 4.06 which does not deviate much from BCS value. Superconductivity in $\mathrm{NdO}_{1-x} \mathrm{~F}_{x} \mathrm{BiS}_{2}$ is easily destroyed when a magnetic field, $\mathrm{H}$, is applied parallel to the $c$-axis while superconductivity survives when $\mathrm{H}$ is applied parallel to the $a b$-plane which gives rise to a large magnetic anisotropy $\gamma=\mathrm{H}^{a b}{ }_{c 2} / \mathrm{H}^{c}{ }_{c 2} \sim 30-40$, comparable to that of high $\mathrm{T}_{c}$ superconductors, the Bi-based cuprates. [23, 59, 78] $\mathrm{H}_{c 2}$ is the upper critical field. Furthermore, in $\mathrm{NdO}_{1-x} \mathrm{~F}_{x} \mathrm{BiS}_{2}$, the normal state after the suppression of superconductivity is semiconducting, where in the BCS picture it is metalic. Therefore, $\mathrm{NdO}_{1-x} \mathrm{~F}_{x} \mathrm{BiS}_{2}$ is an interesting system to investigate the new kind of phonon based superconductivity in the $\mathrm{BiS}_{2}$ superconductors.

Equivalently to other $\mathrm{BiS}_{2}$ based superconductors, in $\mathrm{NdO}_{1-x} \mathrm{~F}_{x} \mathrm{BiS}_{2}$, the superconducting sheets consist of $\mathrm{BiS}_{2}$ square pyramids with corner (S1) and apical (S2) sulfur atoms as shown in Fig 5.2. Doped NdO planes play the role of carrier storages while $\mathrm{Bi} S_{2}$ planes are the active planes in superconductivity. Solubility limit is reached at $x=0.5$ and many groups report that the structure does not form for $x \leq 0.1$.[125] The superconductivity is very sensitive to the sample preparation temperatures, e.g $\mathrm{NdO}_{0.8} \mathrm{~F}_{0.2} \mathrm{BiS}_{2}$ prepared under $700^{\circ} \mathrm{C}$ using solid state reaction method is not superconducting while superconductivity emerges when it is prepared under $800^{\circ}$ C. [125] Similarly to the other superconductors of the class, Nd system also shows enhanced superconductivity upon high pressure annealing. Therefore, the superconductivity of this system requires optimization that is strongly related with the structure. Furthermore, the Debye temperature of this system is $\sim 190 \mathrm{~K}[116]$ indicating a softer lattice compared to $\mathrm{LaO}_{1-x} \mathrm{~F}_{x} \mathrm{BiS}_{2} \cdot[114]$ 
To this end, the crystal structure and the local structure were investigated in an attempt to relate the structural properties and superconducting mechanism of the class.

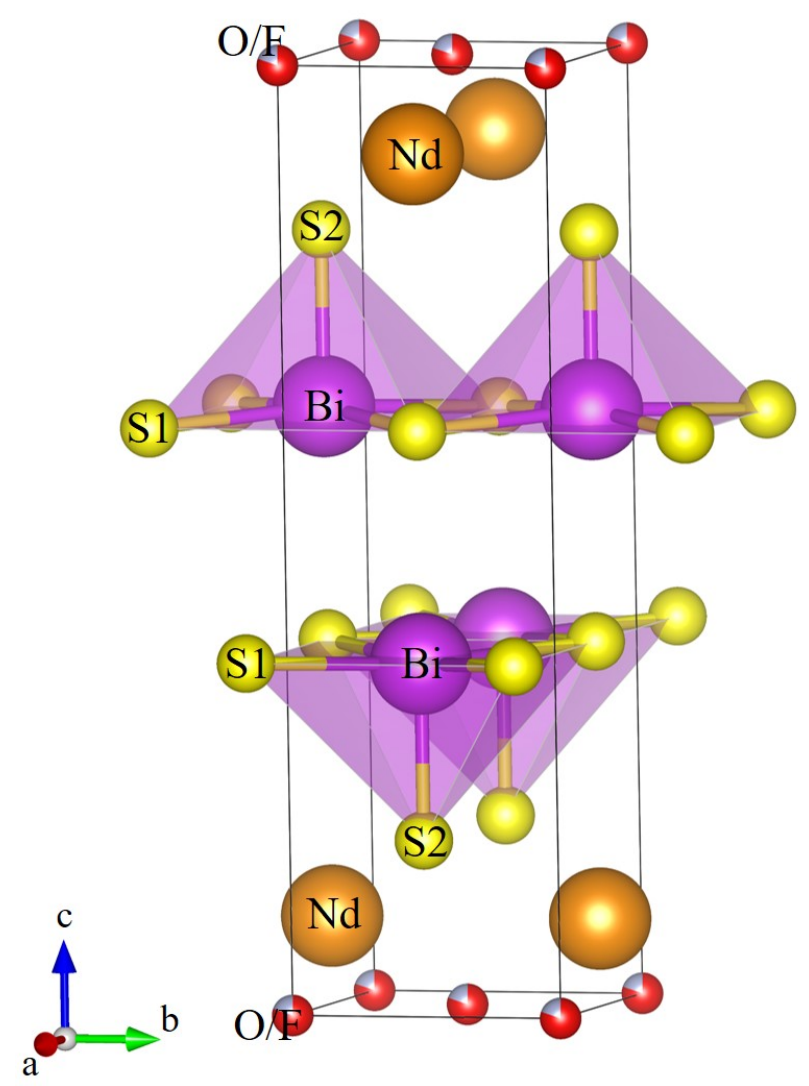

Figure 5.2: The unit cell of $\mathrm{NdO}_{1-x} \mathrm{~F}_{x} \mathrm{BiS}_{2}$ with layers containing $\mathrm{BiS}_{2}$ polyhedra. 


\subsection{Crystal structure of $\mathrm{NdO}_{1-x} \mathrm{~F}_{x} \mathrm{BiS}_{2}$}

The crystal structure refinement of $\mathrm{NdO}_{1-x} \mathrm{~F}_{x} \mathrm{BiS}_{2}$ was carried out in reciprocal space using Rietveld method for $x=0.2$ and 0.4 for temperatures ranging from $2-300 \mathrm{~K}$ and 2-150 K respectively. For both superconducting and non-superconducting compositions, the average structure over the entire temperature range can be well described using $P 4 / n m m$ symmetry. Bi lies at the center of the pyramid base and make equal correlations with S1 sulfur on plane. At $2 \mathrm{~K}, \mathrm{~S} 1$ makes bond lengths of 2.8168 and 2.8204 with Bi in $x=0.2$ and 0.4 compositions respectively. The results of the Rietveld refinement as a function of temperature are summarized in Table 1 and 2 for $x=0.2$ and 0.4 , respectively. $R_{w p}$ and $\chi^{2}$ correspond to the weighted profile $R$ factor and chi-square, respectively. All sites have a multiplicity of 2 . Therefore, a single unit cell contains 10 atoms which will be divided by the unit cell volume to get the number density of $0.0466 \AA^{-3}$. The z-value of each atom can be refined except for $\mathrm{O} / \mathrm{F}$ which is at a special site. The isotropic thermal factors are indicated by $\mathrm{U}_{\text {iso }} . \mathrm{U}_{i s o}=\left\langle u^{2}\right\rangle$ where $\left\langle u^{2}\right\rangle$ is the mean squared displacement from the equillibrium position of the atom due to thermal effects. The numbers in the parentheses correspond to one standard deviation in the mean value of the specific parameter.

The fitting of the diffraction pattern with the calculated model using $P 4 / \mathrm{nmm}$ symmetry is shown in Figs. 5.3(a) and (b), for $x=0.2$ and 0.4 at $2 \mathrm{~K}$, respectively. The data points are represented by black squares and the calculated intensity is represented with a solid red line. The difference between the data and calculated intensities is represented by a solid blue line. The NOMAD is an instrument with medium-range resolution $\left(\frac{\Delta d}{d} \sim 0.8 \%\right)[126]$ and within this resolution, no Bragg peak broadening is observed in either $x=0.2$ or 0.4 composition, unlike the case of high pressure annealed $\mathrm{LaO}_{1-x} \mathrm{~F}_{x} \mathrm{BiS}_{2}$ 


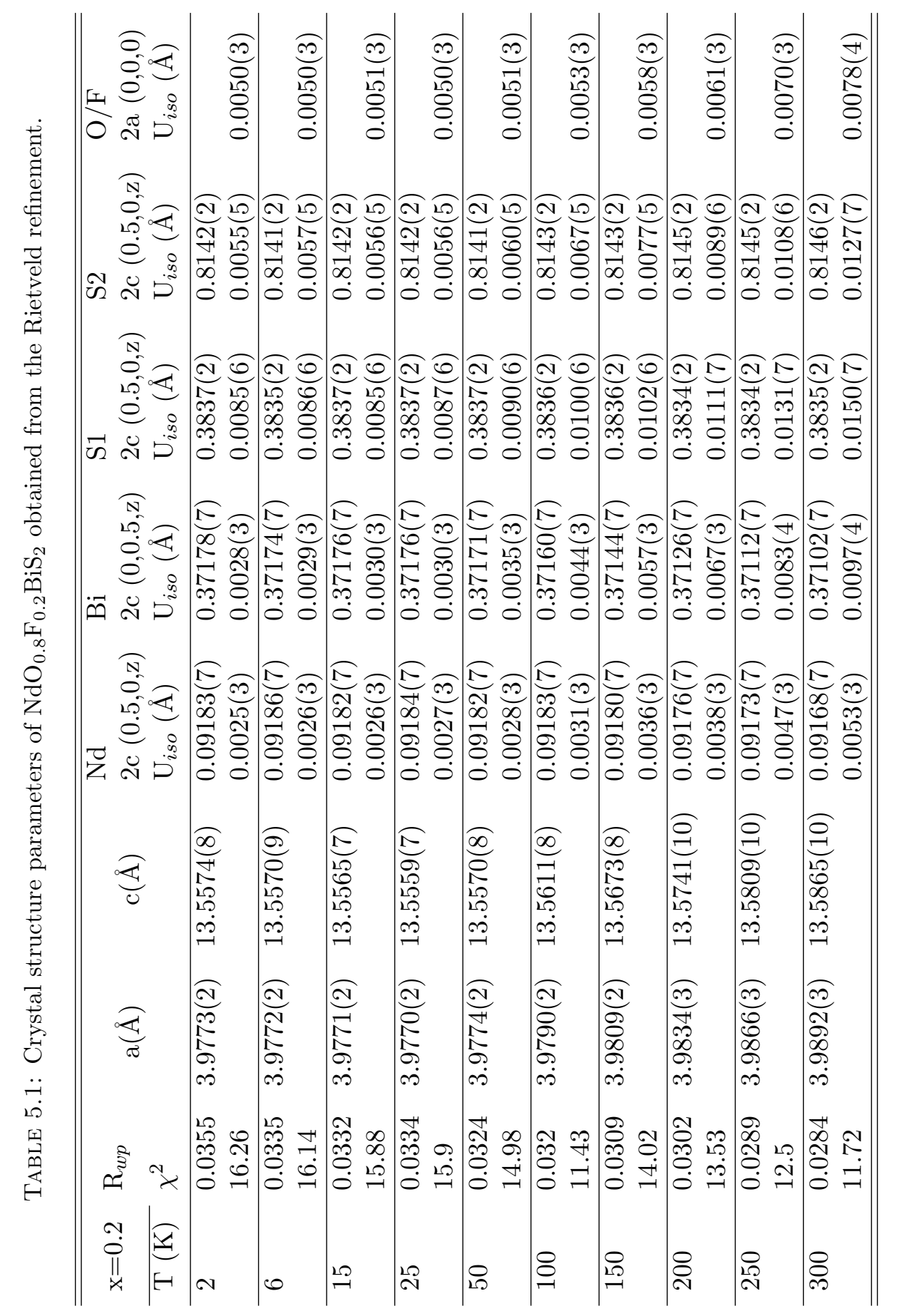




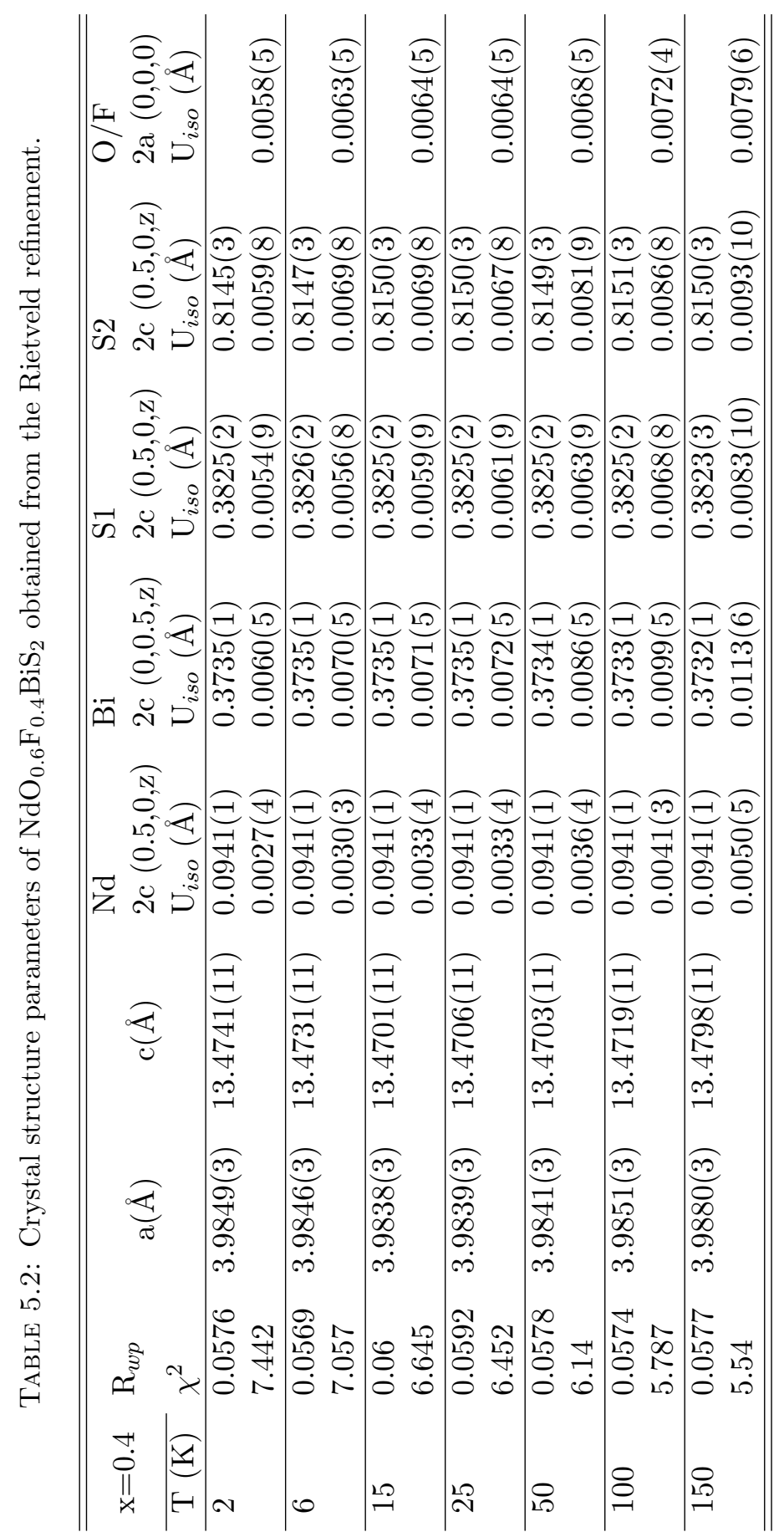


in which $(00 l)$ peaks were severely compromised. [113, 127] If $\mathrm{NdO}_{1-x} \mathrm{~F}_{x} \mathrm{BiS}_{2}$ has less or no structural distortions in local scale compared to $\mathrm{LaO}_{1-x} \mathrm{~F}_{x} \mathrm{BiS}_{2}$, a considerable contribution to the Bragg peak broadening must be coming from the alreday identified structural distortions [127] of $\mathrm{LaO}_{1-x} \mathrm{~F}_{x} \mathrm{BiS}_{2}$. If not, the identified structural distortions must have only accounted for peak broadening partially. 


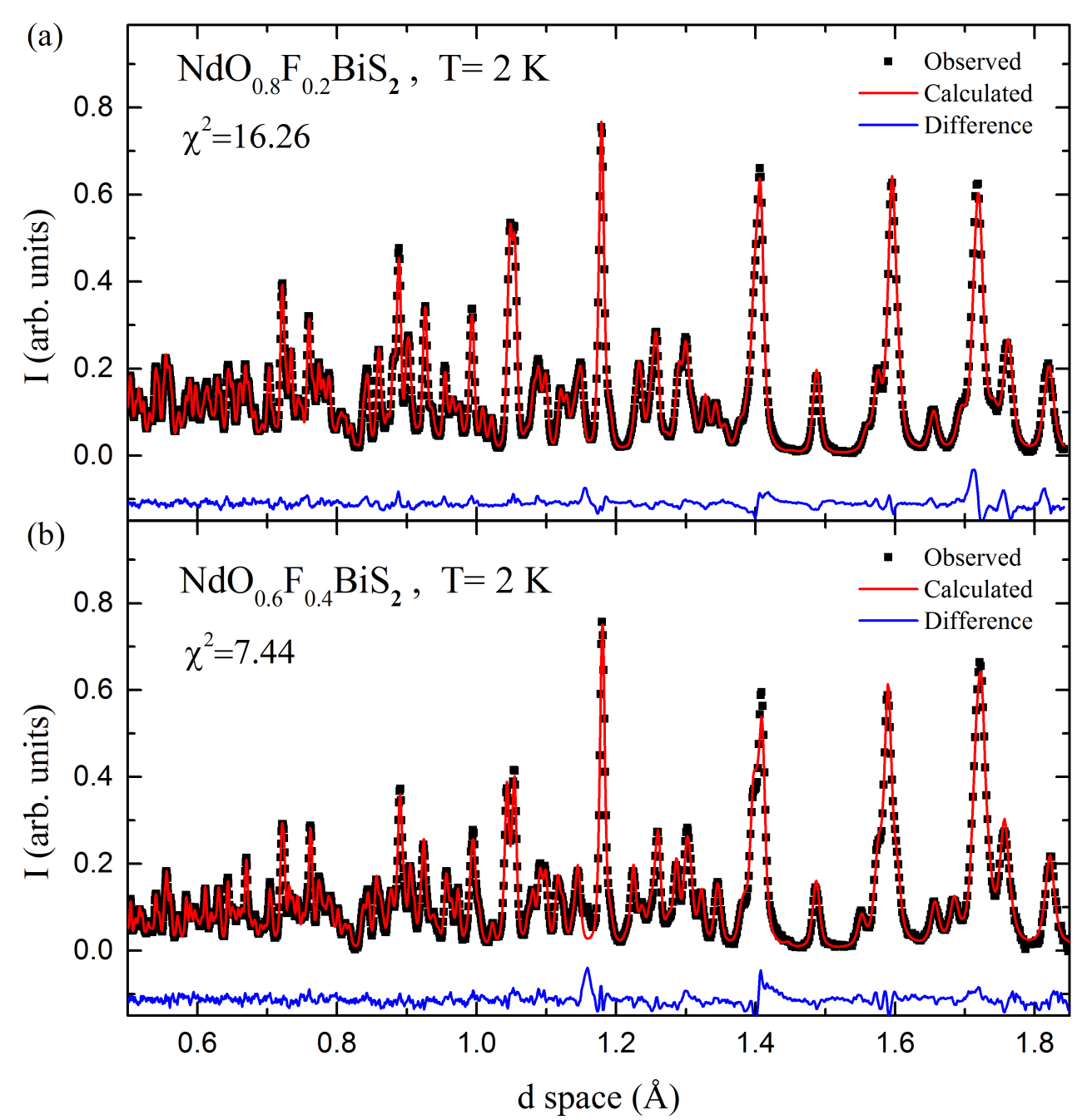

FiguRE 5.3: The neutron powder diffraction pattern of $\mathrm{NdO}_{1-x} \mathrm{~F}_{x} \mathrm{BiS}_{2}$ for (a) $x=0.2$ and (b) $x=0.4$. 
The $a$ and $c$ lattice constants as a function of temperature for $\mathrm{NdO}_{1-x} \mathrm{~F}_{x} \mathrm{BiS}_{2}$ at $x=0.2$ and 0.4 are shown in the Fig. 5.4 (a) and (b). The $a$ lattice constant expands slightly and the $c$ lattice constant contracts considerably. As a result $c / a$ ratio decreases from $c / a=3.4083$ for $x=0.2$ and $c / a=3.3818$ for $x=0.4$ at $2 \mathrm{~K}$. The difference accounts for the slight Bragg peak splitting between the diffraction patterns of the two compositions shown in Fig. 5.3. The data are also compared to the $\mathrm{LaO}_{1-x} \mathrm{~F}_{x} \mathrm{BiS}_{2}$ for $x=0$ and 0.5 in Fig. 5.4 (a) and (b). The $a$ lattice constant of both superconducting and non-superconducting La compositions are larger than that of Nd compositions. The two superconducting samples of La $x=0.5$ and $\mathrm{Nd} x=0.4$ have smaller values for $c$ lattice constant out of the F doped compositions and the smallest value is from the superconductor with the highest $\mathrm{T}_{c}$. Also shown in Fig. 5.4(c) is a plot of the unit cell volume for the Nd and La compositions. A much larger change is observed in the La doped samples than in the $\mathrm{Nd}$ samples with increased $\mathrm{F}$ doping. The significant change of $c$ lattice constant accounts for this larger change in volume of La compositions. It is clear that the lattice contracts with the replacement of smaller ionic radius of $\mathrm{Nd}^{3+}$ for $\mathrm{La}^{3+}$, giving rise to a negative chemical pressure indicated by the unit cell volume compression.

Shown in Figs. 5.5(a) and (b) are plots of the isotropic thermal factors, $\mathrm{U}_{i s o}$, as a function of temperature, for $x=0.2$ and 0.4 , respectively. Similar trends are observed in both compositions except for the $\mathrm{Bi}-\mathrm{U}_{\text {iso }}$ which is larger in the superconducting sample and the $\mathrm{S} 1-\mathrm{U}_{\text {iso }}$ which is larger in the non-superconducting sample. As the $\mathrm{U}_{\text {iso }}$ correspond to the static displacements of atoms, the difference of largest value coming from different atoms in the two compositions imply that the dominant atomic displacements might differ with the doping level. 

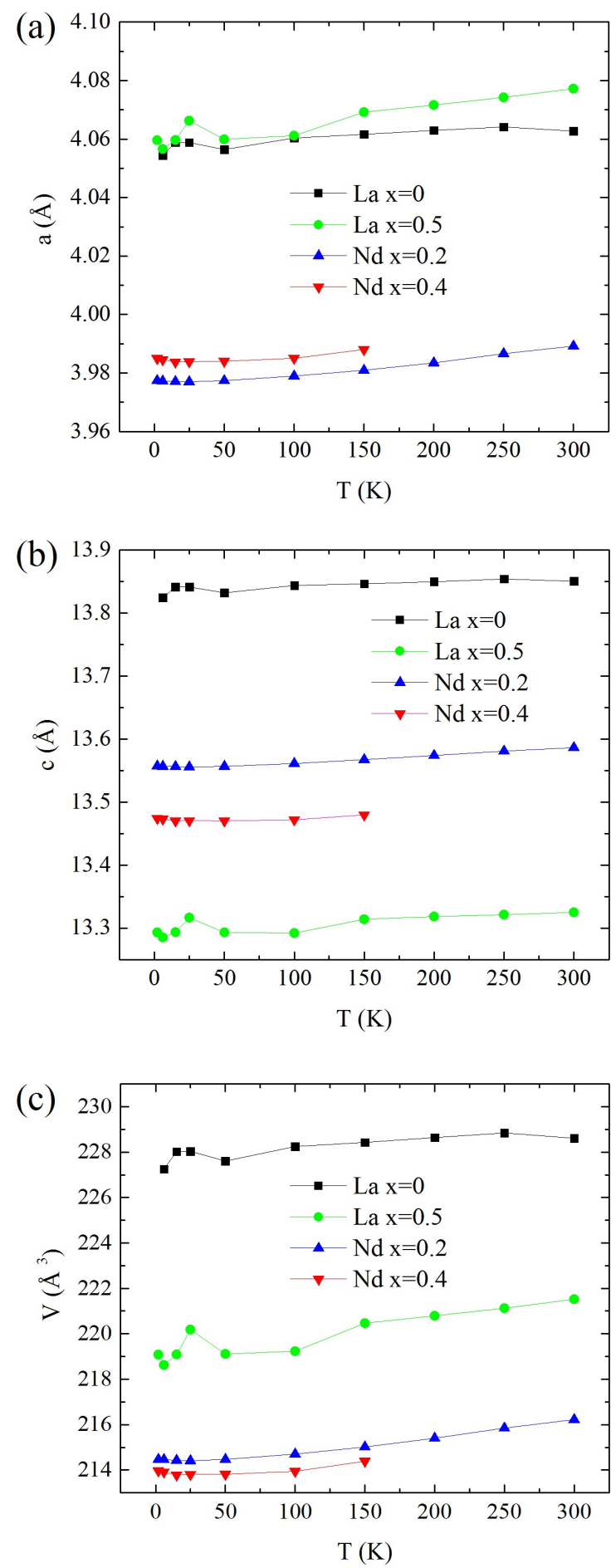

FiguRe 5.4: The lattice parameters (a) $a$ and (b) $b$, and (c) unit cell volume of $\mathrm{ReO}_{1-x} \mathrm{~F}_{x} \mathrm{BiS}_{2}$ for $\mathrm{Re}=\mathrm{Nd}$ and La. 
(a)

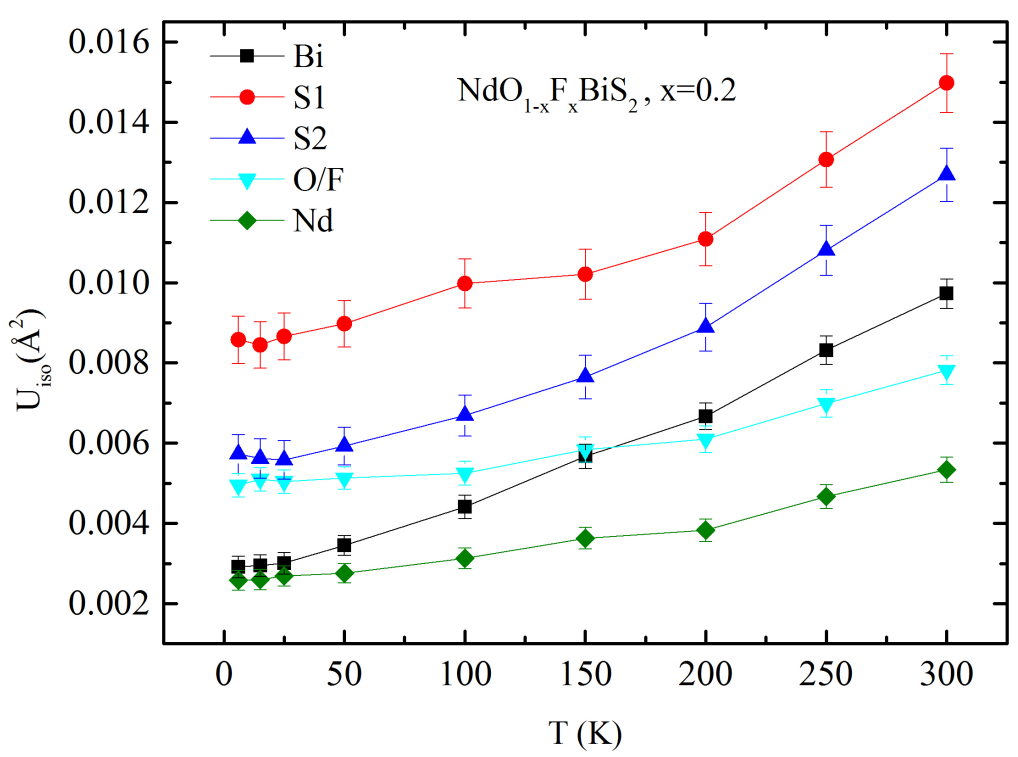

(b)

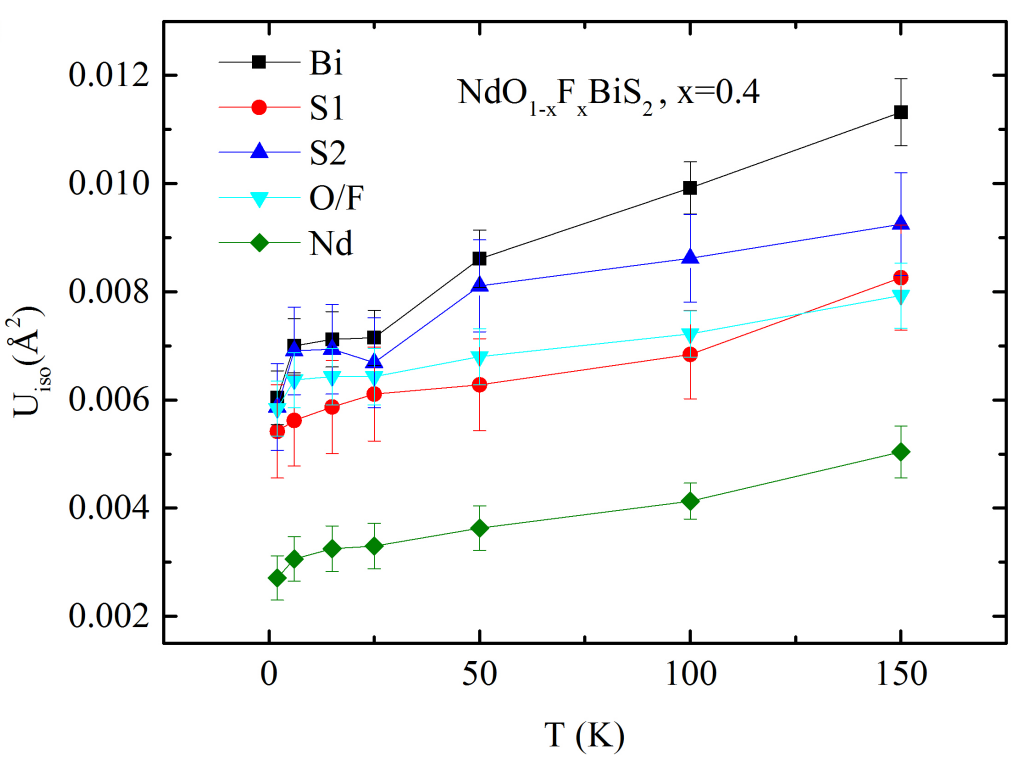

FiguRE 5.5: The isotropic thermal factors of $\mathrm{NdO}_{1-x} \mathrm{~F}_{x} \mathrm{BiS}_{2}$ is shown for (a) $x=0.2$ and (b) $x=0.4$. 


\subsection{The local structure of $\mathrm{NdO}_{1-x} \mathrm{~F}_{x} \mathrm{BiS}_{2}$}

\subsubsection{Composition dependence of local structure}

The PDFs corresponding to the local structures of $x=0.2$ and $x=0.4$ are plotted in

Fig. 5.6. While their local structures are quite similar overall, differences can be seen between the two, especially in the 2.5-3.5 A range that cannot be accounted for by the substitution of $\mathrm{F}$ for $\mathrm{O}$ given their close ionic sizes and neutron scattering lengths. The $\mathrm{PDF}$ in the specific region is a constituent of $\mathrm{Bi}-\mathrm{S} 1, \mathrm{Nd}-\mathrm{S} 2, \mathrm{O} / \mathrm{O}$ and $\mathrm{O}-\mathrm{S} 2$ correlations as labeled in the figure.

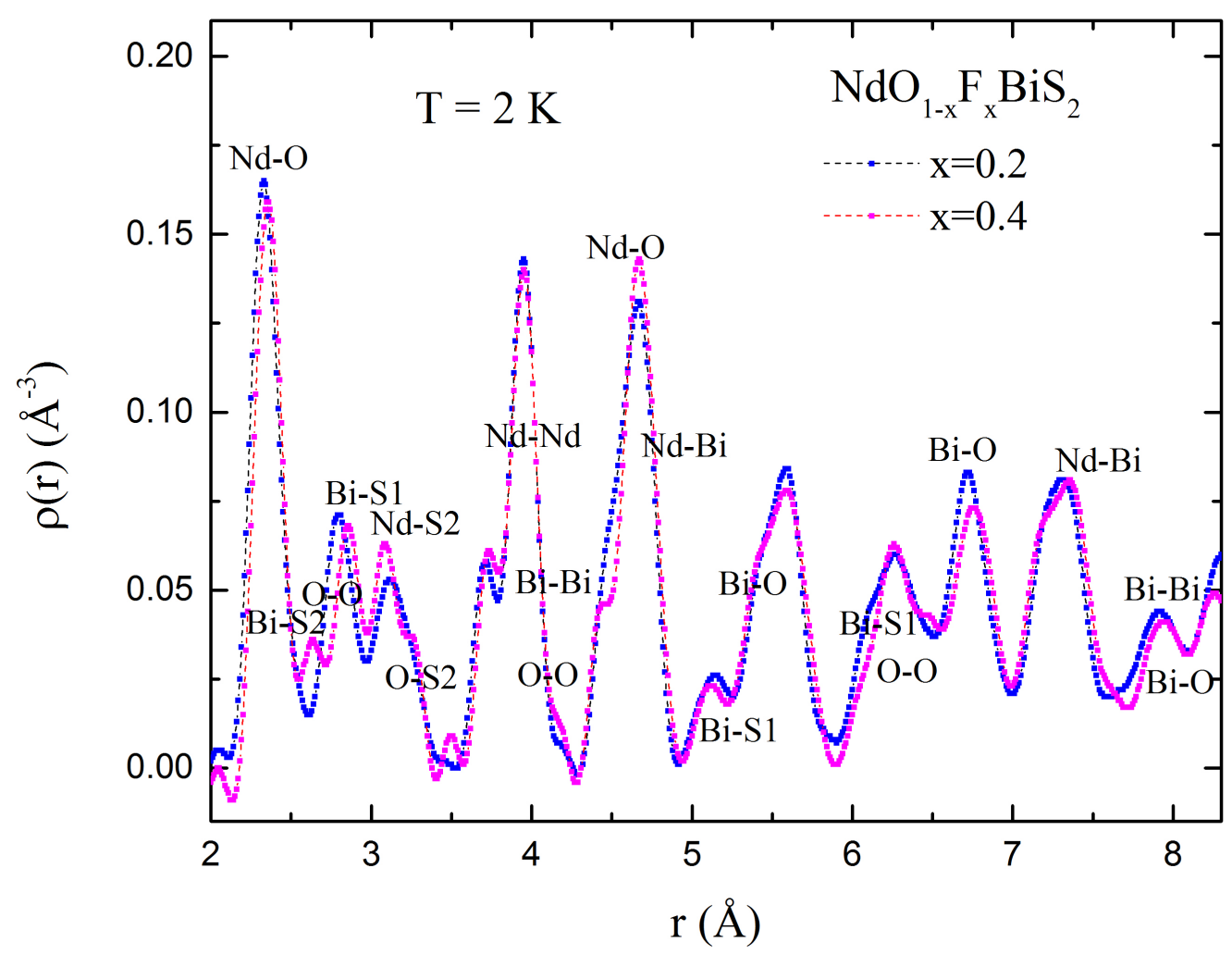

Figure 5.6: A comparison of the local structural data at $x=0.2$ and $x=0.4$ at $2 \mathrm{~K}$. 


\subsubsection{Nature of lattice distortions in $\mathrm{NdO}_{0.8} \mathrm{~F}_{0.2} \mathrm{BiS}_{2}$}

As the initial step, the $x=0.2$ data is compared to a model PDF calculated using the atomic coordinates and unit cell dimensions obtained from the Rietveld refinement corresponding to the average structure. As shown in Fig. 5.7, overall, the average model $\rho_{\text {mod }}(\mathrm{r})$ for the $x=0.2$ in the blue dashed line is in good agreement with the experimental PDF, $\rho_{\text {exp }}$, except in the 2.5-3.5 $\AA$ region where the model peak intensity is higher. The agreement factor, $A$, between the data and the average model is 0.181 . On the other hand, a local model, $\rho_{\text {mod }}$, involving in-plane displacements at both sulfur, S1 and S2 atoms, reproduces the data well as seen in the figure. The A-factor in this case is reduced to $16.2 \%$ and the $\rho_{\text {mod }}$ is the red solid line in Fig. 5.7. Significant improvement is observed in the 2.5-3.5 $\AA$ range upon applying the in-plane displacements of sulfur. To reproduce the data, the S1 and S2 are displaced by $\sim 0.15 \AA$ and $\sim 0.11 \AA$ respectively, in either the $\mathrm{x}$ or $\mathrm{y}$ directions away from their equilibrium positions. The partial PDFs corresponding to Bi-S1 pair correlation shown in Fig. 5.8 demonstrates how the biggest changes observed in the 2.5-3.5 $\AA$ range arise. The single peak at $2.8168 \AA$ in the average structure is locally split to two thus lowering the single peak height.

There are four planes containing sulfur: S2 at $\sim 0.2 \mathrm{c}$ and $0.8 \mathrm{c}$ and $\mathrm{S} 1$ at $\sim 0.4 \mathrm{c}$ and $0.6 \mathrm{c}$ as shown in Fig. 5.9. The in-plane S1 motion gives rise to two different Bi-S1 bond lengths on each layer at $\sim 0.4 \mathrm{c}$ and $0.6 \mathrm{c}$. The magnitude of the two bond lengths are $2.92 \AA$ and $2.71 \AA$ of which the average $2.815 \AA$ is close to the bond length given by the average model. the Considering the displacement direction of the $\mathrm{S} 1$, the displacements can take several modes which will be equivalent to one of $(\mathrm{x}, \mathrm{x}),(\mathrm{x},-\mathrm{x})$ or $(-\mathrm{x},-\mathrm{y})$ modes given the tetragonal symmetry. The two coordinates in each parenthesis refer to the direction of motion of S1 in the two layers. One possible local model of $(-\mathrm{x},-\mathrm{y})$ mode is shown in 


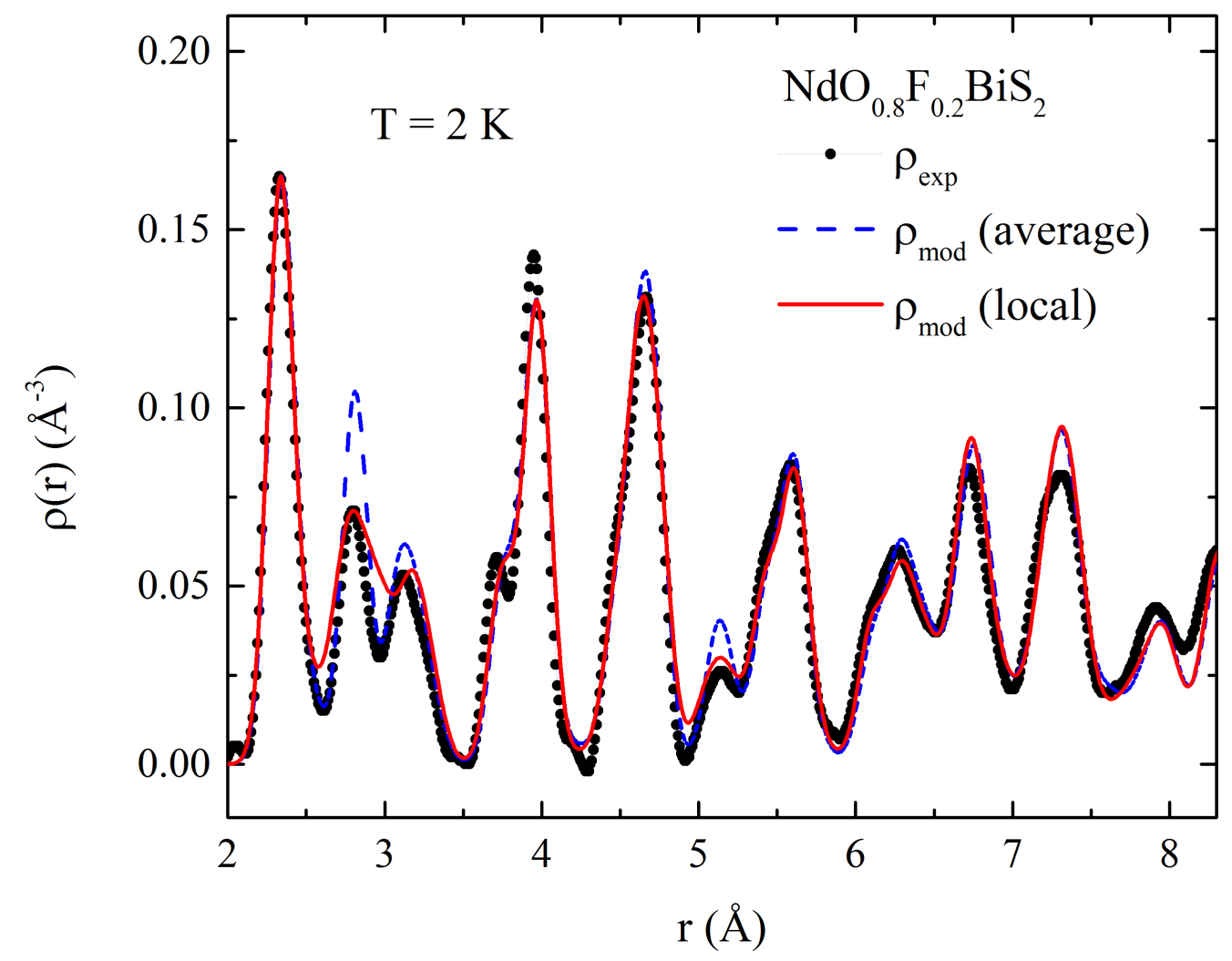

FIGURE 5.7: The comparison of average model (in blue dashed line) and local model (in red solid line) with data represented by black dots in $\mathrm{NdO}_{0.8} \mathrm{~F}_{0.2} \mathrm{BiS}_{2}$

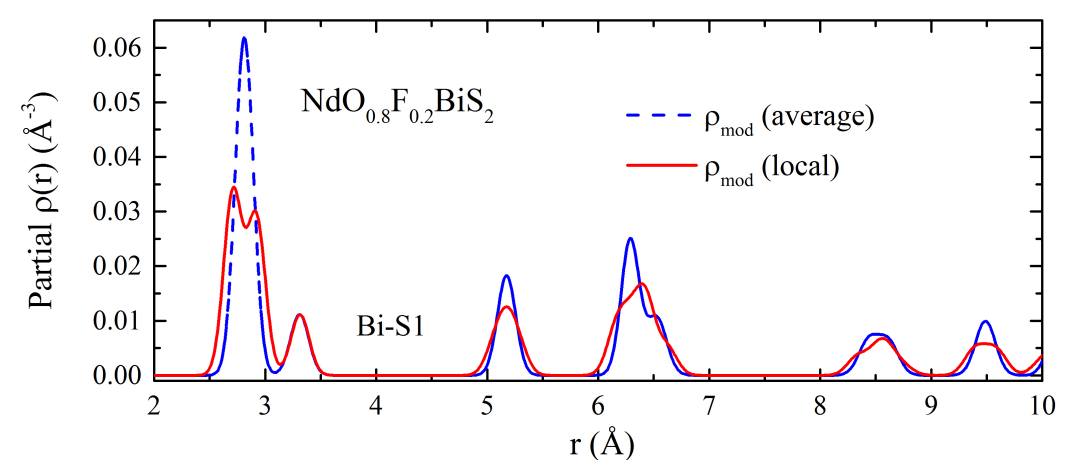

FIGURE 5.8: The partial pair density function of Bi-S1 is shown for $x=0.2$ in $\mathrm{NdO}_{1-x} \mathrm{~F}_{x} \mathrm{BiS}_{2}$. 
Fig. 5.10 where the $\mathrm{S} 1$ atoms at $\mathrm{z} \sim 0.6 \mathrm{c}$ are displaced in $-\mathrm{x}$ direction and at $\mathrm{z} \sim 0.4 \mathrm{c}$, they are displaced in the -y direction. The in-plane $(-\mathrm{x},-\mathrm{y})$ mode is not uniquely determined from the powder data because the model only takes into account the magnitude of the bond but not the direction. Thus, in this case, equivalent models with $(+\mathrm{x},-\mathrm{y}),(+\mathrm{x},+\mathrm{y})$ and $(-\mathrm{x},+\mathrm{y})$ can all produce similar results. These displacements break the four-fold, two fold and inversion symmetries of the $P 4 / n m m$ symmetry. In addition, in-plane type displacements of $\mathrm{S} 2$ is also present on $\sim 0.2 \mathrm{c}$ and $0.8 \mathrm{c}$ planes. Similarly to the S1 modes, S2 also can have different directions of motions in the planes, hence in combination to the $\mathrm{S} 1$ displacements, increases the number of possible modes.

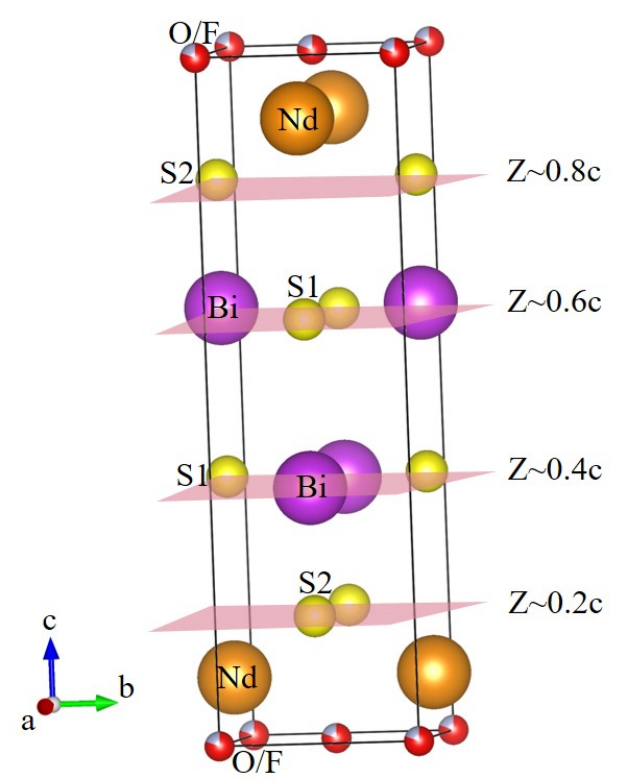

Figure 5.9: The Bi-S planes at $\mathrm{z} \sim 0.2 \mathrm{c}, 0.4 \mathrm{c}, 0.6 \mathrm{c}$ and $0.8 \mathrm{c}$. 
(a) $\mathrm{x}=0.2$

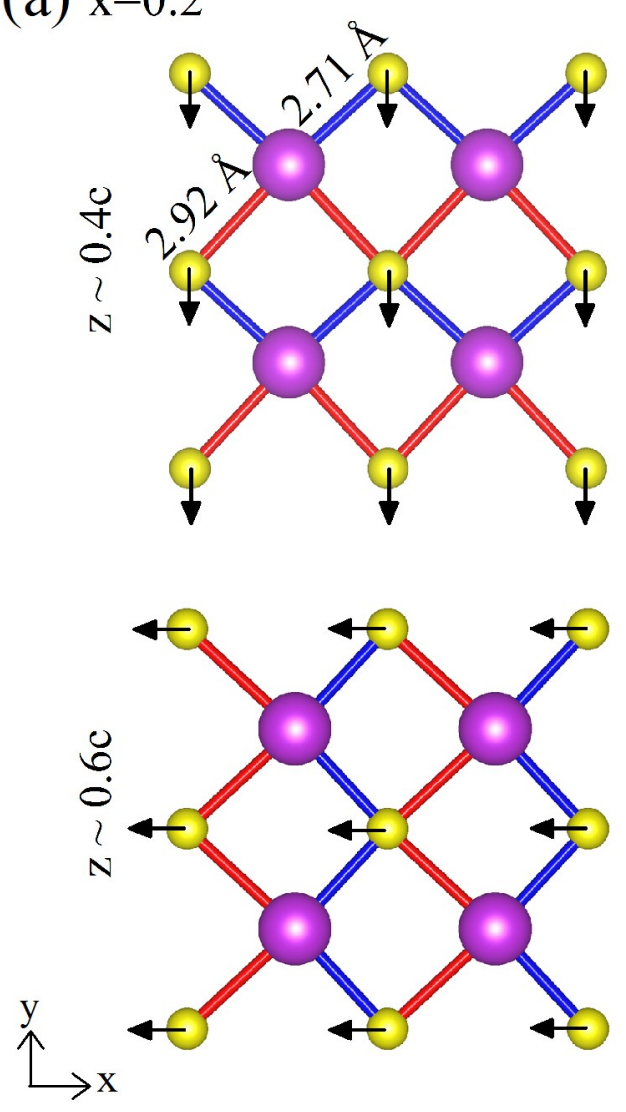

(b) $\mathrm{x}=0.2$

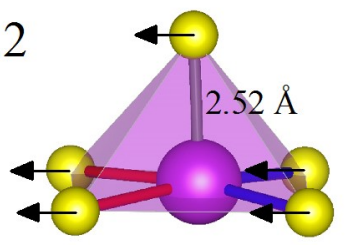

Figure 5.10: The schematics of $\mathrm{BiS}_{2}$ planes at $2 \mathrm{~K}$ depicting the $\mathrm{S} 1$ and $\mathrm{Bi}$ motion in $\mathrm{NdO}_{1-x} \mathrm{~F}_{x} \mathrm{BiS}_{2}$, two magnitudes of bond length at $x=0.2$. (b) Depicts the in-plane and out of plane displacements of $\mathrm{S}$ and $\mathrm{Bi}$ at $x=0.2$. 


\subsubsection{Nature of lattice distortions in $\mathrm{NdO}_{0.6} \mathrm{~F}_{0.4} \mathrm{BiS}_{2}$}

To investigate the impact of fluorine doping level on the structural distortions and superconductivity of superconducting $\mathrm{NdO}_{0.6} \mathrm{~F}_{0.4} \mathrm{BiS}_{2}$, the PDF was analyzed. The $x=0.4$ data is compared to a model PDF corresponding to the average structure for the $x=0.4$ composition at $2 \mathrm{~K}$ and shown in the blue dashed line in Fig. 5.11. Just as in $x=0.2$, the average model cannot adequately describe the intensity of the peaks in the 2.5-3.5 $\AA$ range. To fit the data, a local model $\rho_{\text {mod }}$ that takes into account in-plane atomic displacements of $\mathrm{S} 1, \mathrm{~S} 2$ and $\mathrm{Bi}$ and $c$-axis displacement of $\mathrm{S} 2$ is used to fit the data and can reproduce the local atomic structure well, as shown by the red solid line in Fig. 5.11. Similarly to the $x=0.2$, the $\mathrm{S} 1$ displacement in $x=0.4$ gives rise to two different Bi-S1 bond lengths, which further split with the addition of the Bi displacement as shown in Fig. 5.12(a). The average value of the four Bi-S1 local bond lengths is close to the bond length obtained by average structure refinement. The magnitude of in-plane displacements of Bi, S1 and $\mathrm{S} 2$ at $2 \mathrm{~K}$ are $\sim 0.12,0.21$ and $0.13 \AA$ respectively. The S2 atom is also displaced along c by $0.07 \AA$ as shown in the schematic of the Bi-S tetrahedron in Fig. 5.12(b). The magnitude of the displacements of all atoms is the same at both planes. However, the direction of in-plane type motion of S1 and S2 can still be different giving rise to many possible configurations of bonds just as in the case of $x=0.2$. Bi motion is perpendicular to the S1 motion so that it gives rise to four different bond lengths, which if parallel will give rise to two different bond lengths only and will not make the right intensities. The Bi-S1 partials are calculated from the local model and shown in Fig. 5.13, which show the single peak at $2.8204 \AA$ in the average structure is split in the local $\rho_{\text {mod }}$ represented in the red solid line. 


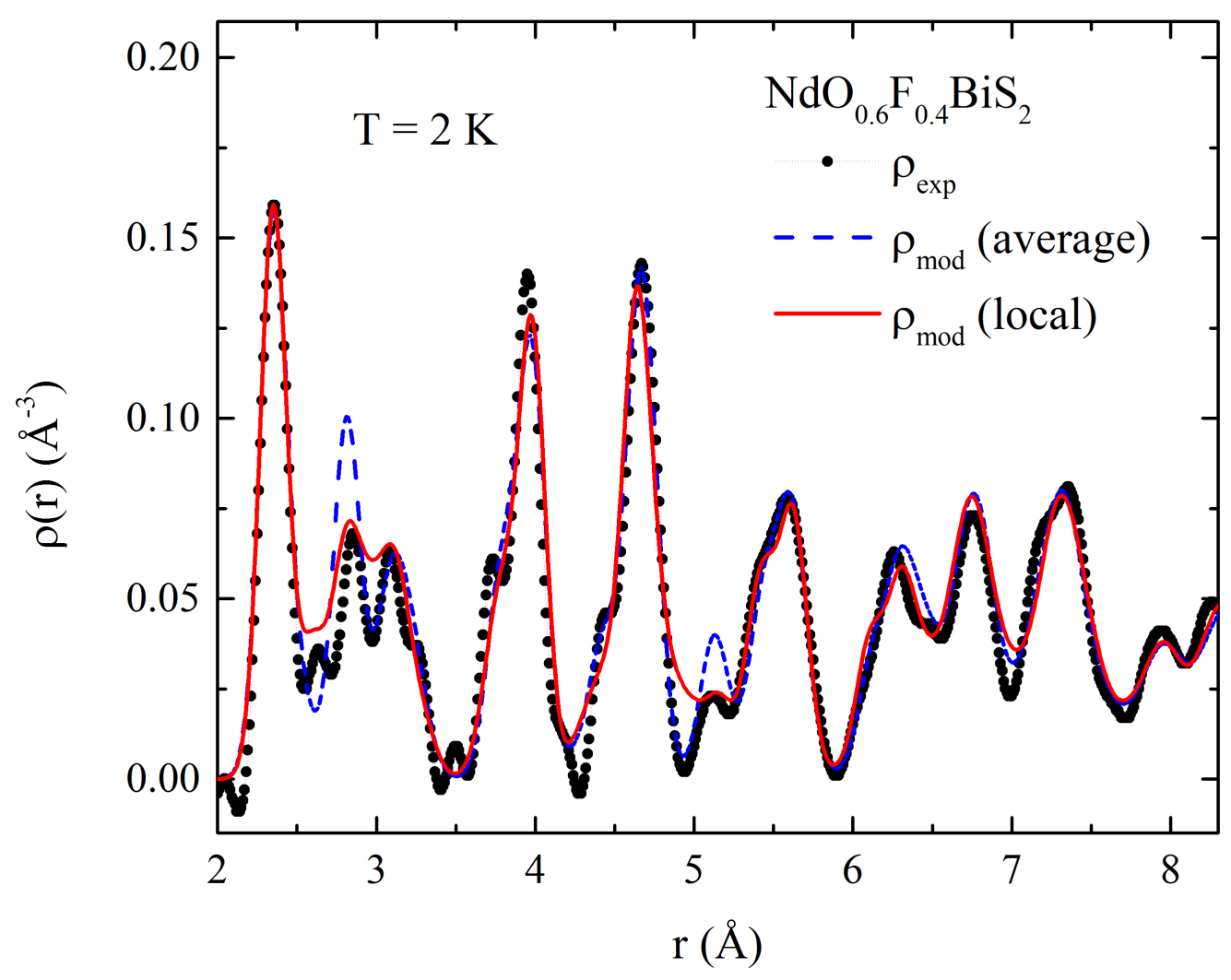

Figure 5.11: The comparison of average model (in blue dashed line) and local model (in red solid line) with data represented by black dots in $\mathrm{NdO}_{0.6} \mathrm{~F}_{0.4} \mathrm{BiS}_{2}$. 
(a) $x=0.4$

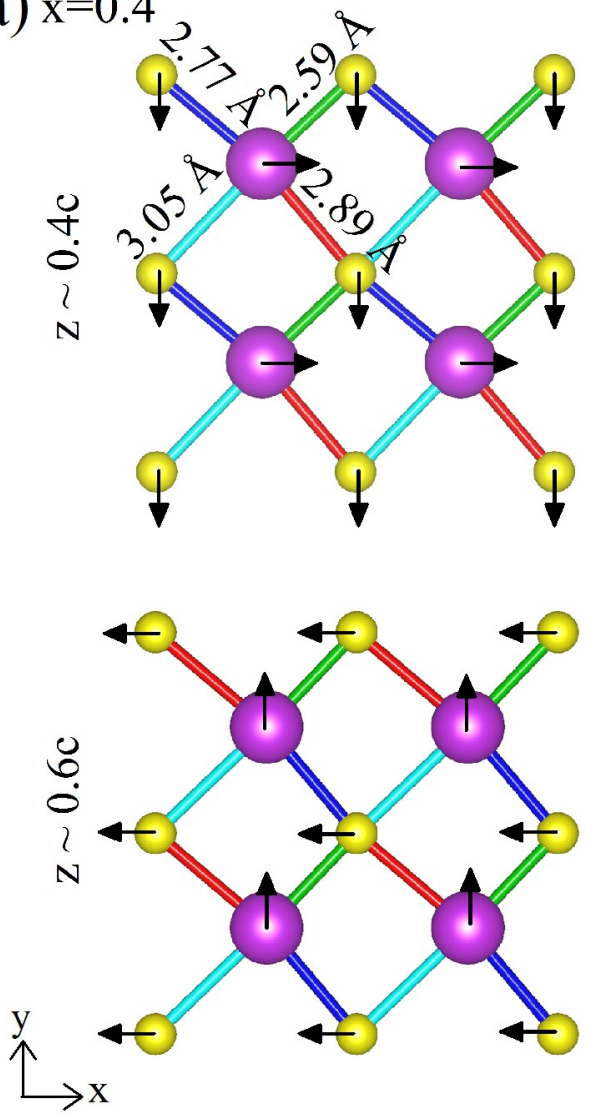

(b) $x=0.4$

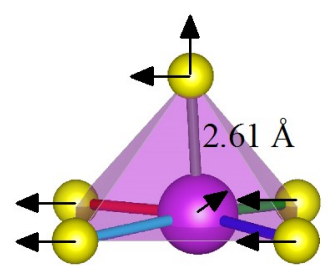

Figure 5.12: The schematics of $\mathrm{BiS}_{2}$ planes at $2 \mathrm{~K}$ depicting the $\mathrm{S} 1$ and Bi motion in $\mathrm{NdO}_{1-x} \mathrm{~F}_{x} \mathrm{BiS}_{2}$, four magnitudes of bond length at $x=0.4$. (b) Depicts the in-plane and out of plane displacements of $\mathrm{S}$ and $\mathrm{Bi}$ at $x=0.4$.

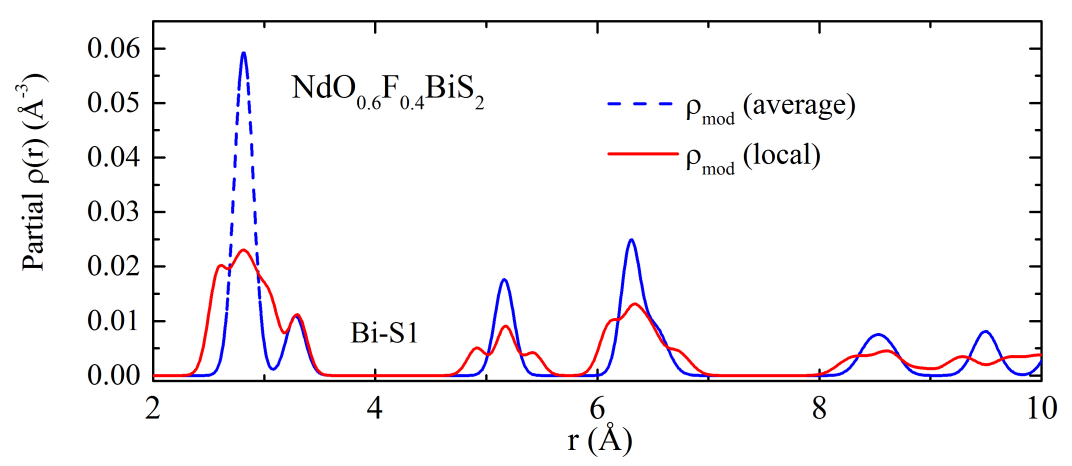

Figure 5.13: The partial pair density function of Bi-S1 is shown for $x=0.4$ in $\mathrm{NdO}_{1-x} \mathrm{~F}_{x} \mathrm{BiS}_{2}$. 

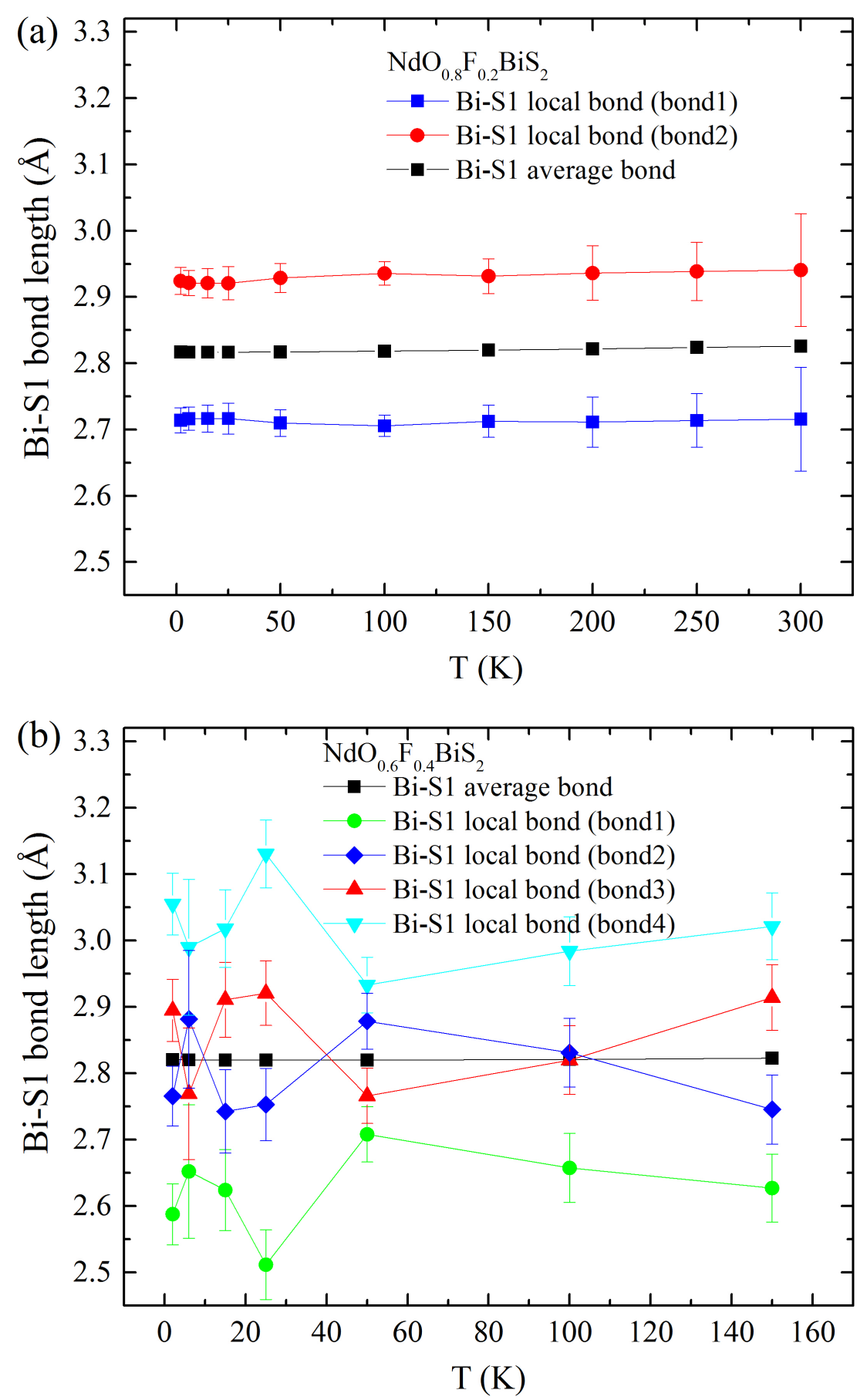

Figure 5.14: The temperature dependence of bond length splitting of $\mathrm{NdO}_{1-x} \mathrm{~F}_{x} \mathrm{BiS}_{2}$ at (a) $x=0.2$ and (b) $x=0.4$. 


\subsubsection{Temperature dependence of bond length splitting}

In $\mathrm{NdO}_{1-x} \mathrm{~F}_{x} \mathrm{BiS}_{2}$, at $x=0.2$, the Bi-S1 bond is split to two different bond lengths due to sulfur movement and at $x=0.4$, it is split to four different bond lengths due to the coordinated displacements of sulfur and $\mathrm{Bi}$. In the non-superconducting $x=0.2$, the bond length splitting does not change with temperature whereas in superconducting $x=0.4$, the splitting increases as as it reaches the $\mathrm{T}_{c}$ as shown in Fig. 5.14(a) and (b), respectively. However, the increase of splitting does not have a smooth transition. It fluctuates considerably below $50 \mathrm{~K}$ within a $0.15 \AA$ A range and stabilizes within $0.05 \AA$ range around the $\mathrm{T}_{c}$. The fluctuation of bond length splitting in the vicinity of superconductivity was similarly observed in the La composition as well, thus indicating the charge fluctuations implied by the splitting play an important role in the superconductivity.

\subsection{Summary}

The crystal structure of $\mathrm{NdO}_{1-x} \mathrm{~F}_{x} \mathrm{BiS}_{2}$ at $x=0.2$ and 0.4 can be well reproduced by $P 4 / n m m$ symmetry. The unit cell of the two Nd doped samples are lower in volume compared to La doped samples thus confirms the chemical pressure is indeed generated by the smaller ionic radius. The samples are not high pressure annealed and the Bragg peaks do not show any broadening in the two samples. With increasing doping level, the $a$ axis increases little compared to the decrease of $c$ axis of the unit cell. The largest atomic thermal factors of $x=0.2$ and 0.4 come from $\mathrm{S} 1$ and $\mathrm{Bi}$, respectively.

The crystal structure of $\mathrm{NdO}_{1-x} \mathrm{~F}_{x} \mathrm{BiS}_{2}$ is locally distorted. In $x=0.2$, the in-plane sulfur displacement gives rise to an asymmetric bond length environment around Bi making two different bond lengths. In $x=0.4$, in addition to sulfur movement, Bi displacements 
also contribute, hence giving rise to four different bond lengths.[128] Several bond length arrangements are present depending on the direction of atomic movements in the two superconducting planes. The bond length splitting remains same for $x=0.2$ with temperature in $2-300 \mathrm{~K}$ range, however in $x=0.4$ the splitting fluctuates below $50 \mathrm{~K}$ as it approaches the $\mathrm{T}_{c}$. Just like in the case of superconducting sample of La series, $c$-axis motion of apical sulfur is present in the superconducting $x=0.4$. 


\section{Chapter 6}

\section{Ferro-lattice-distortions and charge fluctuations in superconducting $\mathbf{R e O}_{1-x} \mathbf{F}_{x} \mathbf{B i S}_{2}$ $(\mathrm{Re}=\mathrm{La}, \mathrm{Nd}$ and $\mathrm{Pr})$}

\subsection{Introduction}

When superconductivity is mediated by lattice vibrations, the structural features essential to the electron-phonon coupling can be the key to revealing the mechanism $[129,130]$. This is a central issue in the new class of $\mathrm{ReOBiS}_{2}(\mathrm{Re}=\mathrm{La}, \mathrm{Nd}, \mathrm{Pr}$ and $\mathrm{Yb})$ based superconductors $[15,17,88]$ as their layered crystal structures are subject to significant disorder [8]. The superconductivity of these samples are induced via fluorine substitution at oxygen sites. The crystal symmetry of $\mathrm{ReOBiS}_{2}$ systems has long been presumed to be tetragonal, $\mathrm{P} 4 / \mathrm{nmm}$ (space group \#129) [25, 26, 64, 112, 113, 131, 132]. The structure is quasi two dimensional in nature with the superconducting $\mathrm{BiS}_{2}$ bi-layers sandwiched between the insulating $\mathrm{ReO}$ layers. 
However, many theoretical works suggested alternative symmetries with and without doping. From first-principles calculations, it was suggested that the symmetry must be lower, $P 2_{1} m n$ (\#31) for undoped and $P 22_{1} 2$ (\#17) for doped samples. The symmetry lowering was caused by a lattice instability that moves sulfur atoms toward Bi and charge density waves driven by a sinusoidal distortion, in the two cases, respectively. $[15,18]$ Density functional perturbation theory with structural optimization showed that the space group reduces from $P 4 / n m m$ to $P \overline{4} m 2$ (\#115) upon fluorine doping. Meanwhile, band structure and spin polarization calculations proposed two yet different symmetries, the non-centrosymmetric $C 2(\# 5)$ and centrosymmetric $P 2_{1} / m(\# 11)$ based on the likely nature of the atomic distortions induced by the interaction between superconducting layers and ReO layers and electron hybridization in ReO layers. It was shown that these symmetries can lower the total energy with respect to the nominal $P 4 / \mathrm{nmm}$ structure. In addition, as the energy difference between these symmetries are small, it was suggested that the co-existence of these politypes is also probable. $[71,133]$

The Fermi surface of $\mathrm{BiS}_{2}$ superconductors is dominated by the hybridized orbitals of Bi $6 p$ and S 3p. Theoretical works suggested that a large phonon softnening occurs due to the dynamic sulfur displacements, leading to an instability around the $\Gamma$ point in $x=0$, and along the entire line $Q=(\pi, \pi, 0)$ with doping, that has been associated with strong Fermi suface nesting.[15, 17]. Symmetry lowering will have significant implications on the band structure thus experimental verification is necessary. Earlier neutron powder measurements with emphasis on the local atomic structure alluded to sulfur displacements that can possibly lead to Bi charge disproportionation [127] are consistent with theoretical predictions. Single crystal diffraction is clearly needed to resolve 
the symmetry of the $\mathrm{BiS}_{2}$ lattice precisely. To this end, neutron diffraction measurements on non superconducting $\mathrm{LaOBiS}_{2}$ and high energy synchrotron x-ray diffraction measurements on superconducting $(\mathrm{La} / \mathrm{Nd} / \mathrm{Pr}) \mathrm{O}_{1-x} \mathrm{~F}_{x} \mathrm{BiS}_{2}$ single crystal samples were carried to observe the subtle lattice deformations in an attempt to determine the correct symmetry.

\subsection{Symmetry lowering in $\mathrm{LaOBiS}_{2}$}

The observed neutron diffraction pattern of $\mathrm{LaOBiS}_{2}$ on $h k 0$ plane is shown in Fig. 6.1(a) and the labelled Bragg spots correspond to P4/nmm symmetry. It can readily be seen that several Bragg spots (circled) are not reproduced by this long presumed symmetry. Shown in Fig. 6.1(b) is the calculated Bragg pattern in color dots from the $P 4 / n m m$ and the peaks with a higher intensity are indicated in red. Based on its reflection condition in the $h k 0$ plane $(h+k=2 n)$, odd combinations of $h+k$ are forbidden. This is consistently observed in the superconducting crystals as well, as will be shown below, thus the $P 4 / n m m$ symmetry cannot explain the extra reflections observed. The integrated intensity of the neutron data across the $h \overline{3} 0$ line is plotted in Fig. 6.2. The forbidden reflections are much weaker by comparison to the main Bragg peaks. This is also true for Bragg peaks integrated along other crystallographic directions. 


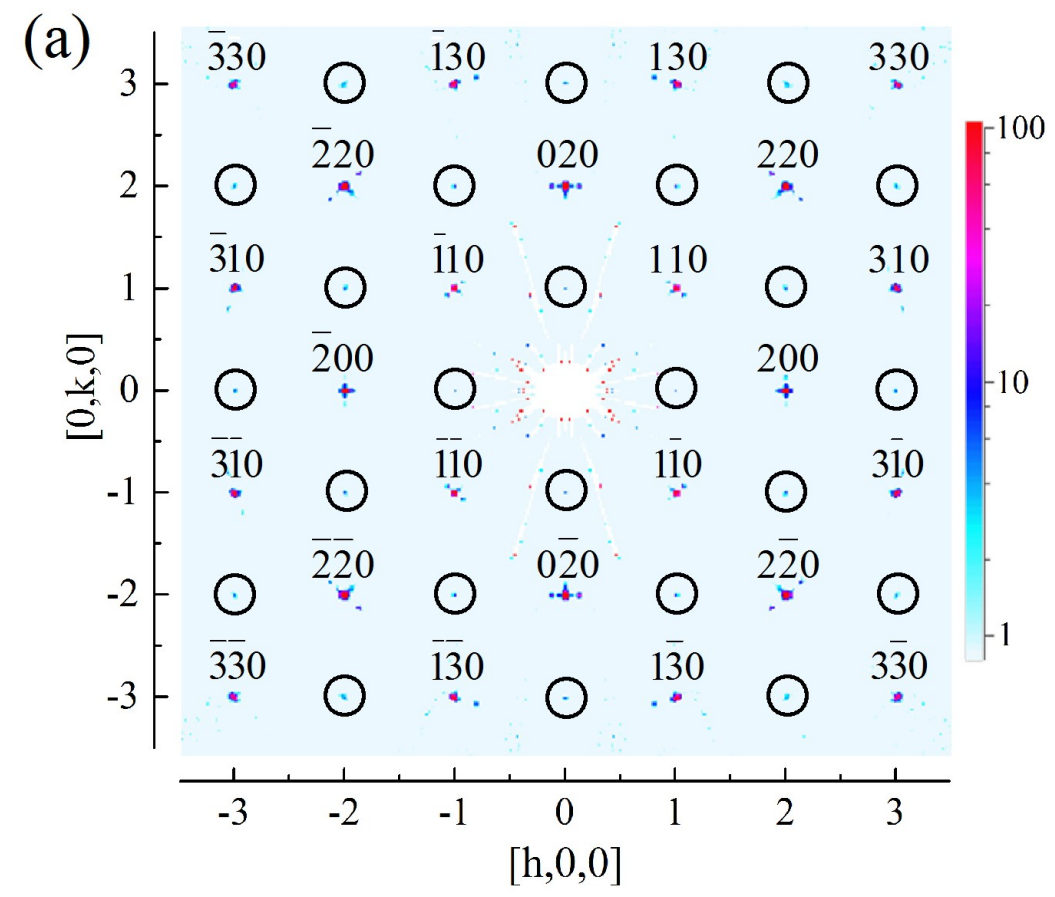

(b)

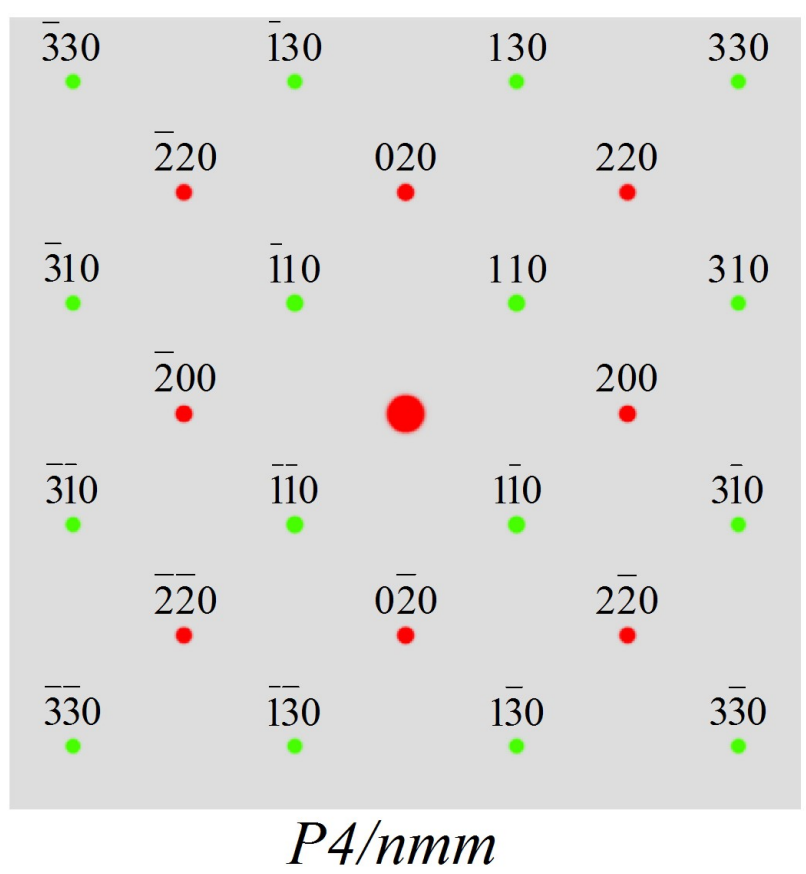

FIGURE 6.1: (a) The single crystal neutron diffraction pattern in the hk0 reciprocal plane for the parent compound, $\mathrm{LaOBiS}_{2}$. In (b) the $h k 0$ plane is constructed using the $P 4 / n m m$ symmetry. 


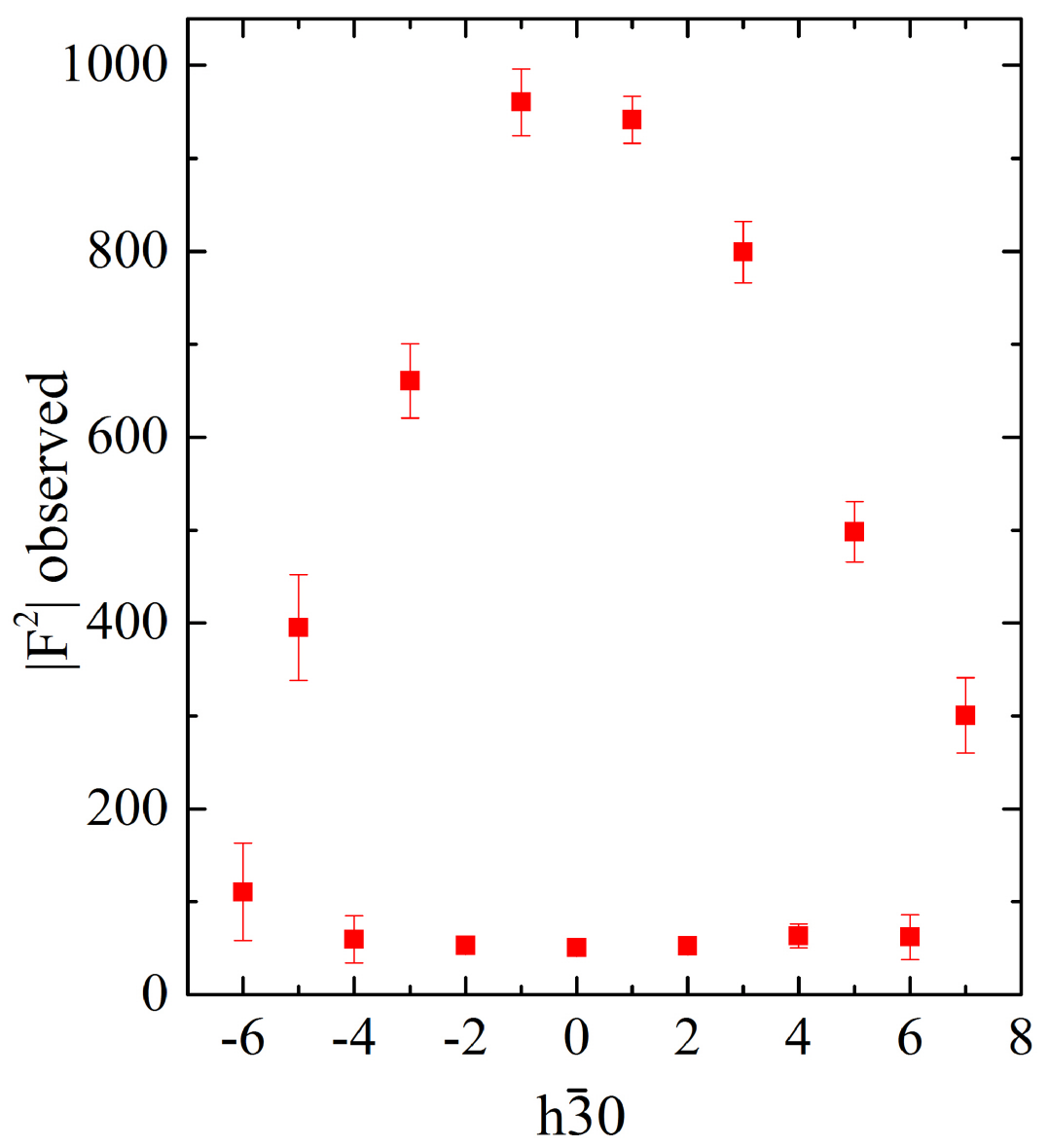

FiguRE 6.2: The integrated intensity of the observed peaks is plotted along the $h \overline{3} 0$ line.

\subsubsection{Testing the theoretically predicted symmetries}

To determine the symmetry that can reproduce the underlying superlattice structure, we first test the previously proposed symmetries. The diffraction patterns from four different symmetries are considered initially; $P 4 m m, P \overline{4} m 2, P 2_{1} m n$ and $P \mathscr{2}_{1} / m$ as shown in Fig. 6.3. The corresponding unit cells are shown in Table 1, 2 and 3, respectively. The $P 4 m m$ and $P \overline{4} m 2$ are subgroups of $P 4 / n m m$. Given that the same reflection conditions apply as in the $P 4 / n m m$, neither symmetry can reproduce the new reflection conditions. The $P 2{ }_{1} m n$ symmetry suggested in Ref. [15], having the same reflection 
condition, cannot reproduce the new reflections either. The comparison with the symmetry, $P 2_{1} / m$, yields a Bragg structure with extra reflections as seen in Fig. 6.3(d). The $P 2_{1} / m$ symmetry has a reflection condition: along the $0 \mathrm{k} 0$ line, $\mathrm{k}=2 \mathrm{n}$. This condition prohibits the symmetry from having several critical reflections at spots circled in the figure. This leads us to conclude that the symmetry of $\mathrm{LaO}_{1-x} \mathrm{~F}_{x} \mathrm{BiS}_{2}$ is considerably lower than expected.

(a)

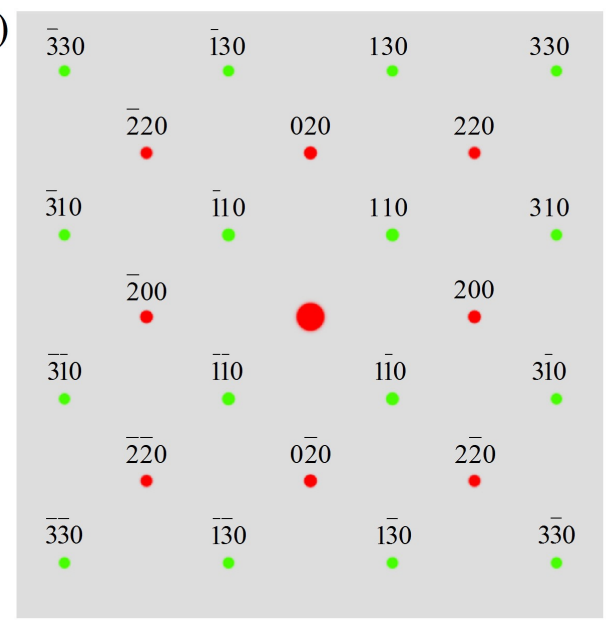

P4mm

(c)

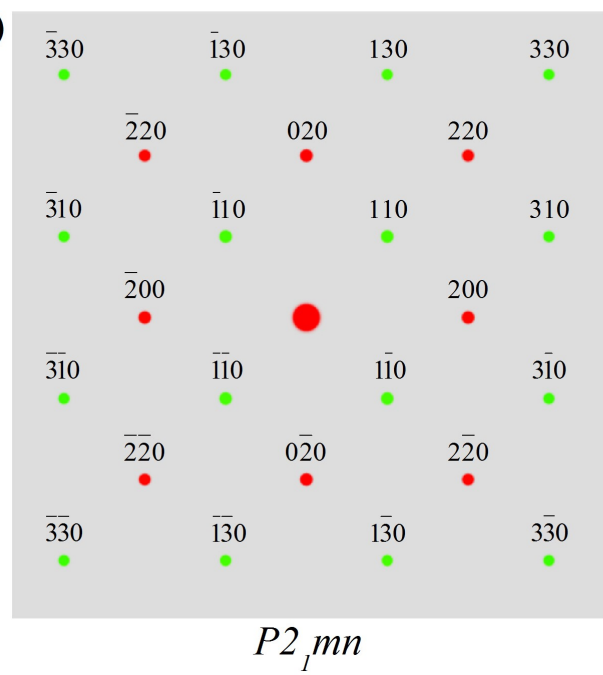

(b)

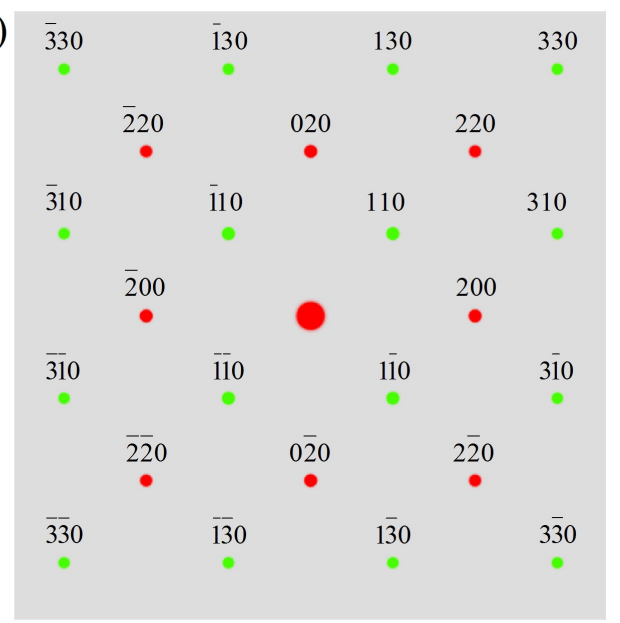

$P \overline{4} m 2$

(d)

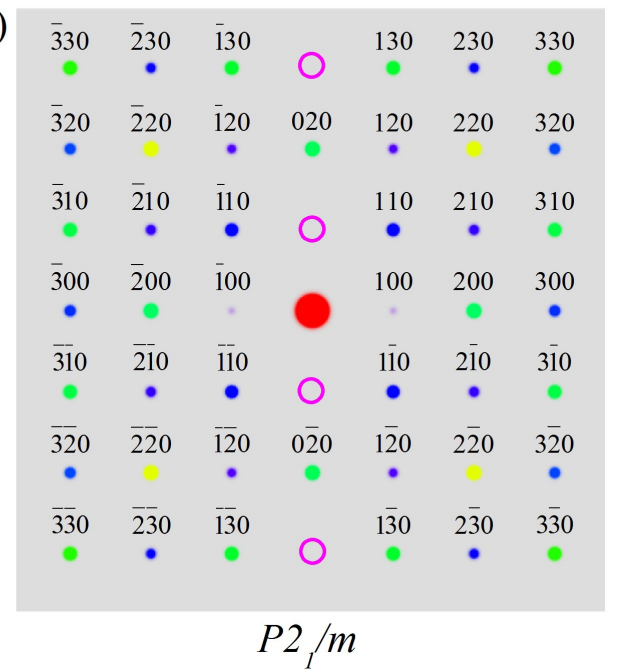

Figure 6.3: The calculated reciprocal patterns based on (a) $P 4 m m$, (b) $P \overline{4} m 2$, (c) $P 2_{1} m n$ and (d) $P 2_{1} / m$ symmetries. 
TABLE 6.1: The tested model of $P 4 / n m m$ symmetry.

\begin{tabular}{|cccc|}
\hline $\mathrm{LaOBiS}_{2}$ & \multicolumn{2}{c|}{$P 4 / n m m$} & $(\# 129)$ \\
\hline & $a(\AA)$ & 4.0544 & \\
& $b(\AA)$ & 13.8246 & \\
& & & \\
& $\mathrm{x}$ & $\mathrm{y}$ & $\mathrm{z}$ \\
$\mathrm{La}$ & 0.5 & 0 & 0.0904 \\
$\mathrm{Bi}$ & 0 & 0.5 & 0.3688 \\
$\mathrm{~S} 1$ & 0.5 & 0 & 0.3832 \\
$\mathrm{~S} 2$ & 0.5 & 0 & 0.8096 \\
$\mathrm{O}$ & 0 & 0 & 0 \\
\hline
\end{tabular}

TABLE 6.2: The tested model of $P 4 m m$ symmetry.

\begin{tabular}{|c|c|c|c|}
\hline \multicolumn{2}{|c|}{$\mathrm{LaOBiS}_{2}$} & \multicolumn{2}{|c|}{$P 4 m m(\# 99)$} \\
\hline & $a(\AA)$ & 4.0544 & \\
\hline & $b(\AA)$ & 13.824 & \\
\hline & $\mathrm{x}$ & $\mathrm{y}$ & $\mathrm{z}$ \\
\hline $\mathrm{La}$ & 0 & 0 & 0.0904 \\
\hline $\mathrm{La}$ & 0.5 & 0.5 & 0.9096 \\
\hline $\mathrm{Bi}$ & 0 & 0 & 0.6312 \\
\hline $\mathrm{Bi}$ & 0.5 & 0.5 & 0.3688 \\
\hline S1 & 0 & 0 & 0.3832 \\
\hline S1 & 0.5 & 0.5 & 0.6168 \\
\hline $\mathrm{S} 2$ & 0 & 0 & 0.8096 \\
\hline $\mathrm{S} 2$ & 0.5 & 0.5 & 0.1904 \\
\hline $\mathrm{O}$ & 0.5 & 0 & 0 \\
\hline
\end{tabular}

TABLE 6.3: The tested model of $P 2{ }_{1} m n$ [15] symmetry.

\begin{tabular}{|c|c|c|c|}
\hline \multicolumn{2}{|c|}{$\mathrm{LaOBiS}_{2}$} & \multicolumn{2}{|c|}{$P 2_{1} m n(\# 31)$} \\
\hline & $a(\AA)$ & 4.037 & \\
\hline & $b(\AA)$ & 4.029 & \\
\hline & $c(\AA)$ & 14.217 & \\
\hline & $\mathrm{x}$ & $\mathrm{y}$ & $\mathrm{Z}$ \\
\hline $\mathrm{La}$ & 0.4997 & 0 & 0.0858 \\
\hline $\mathrm{Bi}$ & 0.4973 & 0 & 0.6324 \\
\hline S1 & 0.525 & 0 & 0.3946 \\
\hline $\mathrm{S} 2$ & 0.4988 & 0 & 0.8102 \\
\hline $\mathrm{O}$ & 0.4996 & 0.5 & 0 \\
\hline
\end{tabular}


TABLE 6.4: The tested model of $P \overline{4} m 2$ [18] symmetry.

\begin{tabular}{|cccc|}
\hline $\mathrm{LaOBiS}_{2}$ & $P \overline{4} m 2(\# 115)$ \\
\hline & $a(\AA)$ & 4.1091 & \\
& $b(\AA)$ & 13.4196 & \\
& & & \\
& $\mathrm{x}$ & $\mathrm{y}$ & $\mathrm{z}$ \\
$\mathrm{La}$ & 0.5 & 0 & 0.1073 \\
$\mathrm{Bi}$ & 0 & 0.5 & 0.3855 \\
$\mathrm{~S} 1$ & 0.5 & 0 & 0.3844 \\
$\mathrm{~S} 2$ & 0.5 & 0 & 0.8128 \\
$\mathrm{O}$ & 0 & 0 & 0 \\
$\mathrm{O}$ & 0.5 & 0.5 & 0 \\
\hline
\end{tabular}

TABLE 6.5: The tested model of $P 2_{1} / m$ [134] symmetry.

\begin{tabular}{|c|c|c|c|}
\hline \multicolumn{2}{|c|}{$\mathrm{LaOBiS}_{2}$} & \multicolumn{2}{|c|}{$P 2_{1} / m(\# 11)$} \\
\hline & $a(\AA)$ & 4.0769 & \\
\hline & $b(\AA)$ & 4.0618 & \\
\hline & $c(\AA)$ & 13.885 & \\
\hline & $\beta\left(^{\circ}\right)$ & 90.12 & \\
\hline & $\mathrm{x}$ & $\mathrm{y}$ & Z \\
\hline La & 0.2496 & 0.25 & 0.0899 \\
\hline $\mathrm{Bi}$ & 0.2346 & 0.25 & 0.6309 \\
\hline S1 & 0.219 & 0.25 & 0.3844 \\
\hline $\mathrm{S} 2$ & 0.2483 & 0.25 & 0.8094 \\
\hline $\mathrm{O}$ & 0.753 & 0.25 & 0.002 \\
\hline
\end{tabular}

\subsubsection{Sulfur distortions as a possible candidate}

It was theoretically suggested that the in-plane sulfur atom, S1, displacements lead to ferroelectric like modes [15] but with no structural transition because of quantum zero-point motions that render the system dynamically disordered. Our earlier neutron powder diffraction measurements yielded a similar outcome but with more sizeable S1 distortions, $\sim 0.3 \AA$ [127]. Moreover, ferro-distortive and antiferro-distortive crystal distortions, in which the two $\mathrm{S} 1$ atoms in the $\mathrm{BiS}_{2}$ bi-layers are displaced simultaneously in the $\mathrm{x}$ - or $\mathrm{y}$-directions, either in parallel or anti-parallel, were proposed but were indistinguishable from the analysis of the powder data as each model resulted in the same magnitude of bonds regardless of orientation in real-space. 
As the second step to find the cause for the underlying superlattice Bragg peak pattern, we consider the proposed sulfur displacements. Within one unit cell, three unique directions of the $\mathrm{S} 1$ displacements are possible as listed here: $(+\mathrm{x},+\mathrm{x}),(+\mathrm{x},+\mathrm{y})$ and $(+\mathrm{x},-\mathrm{x})$ where the two coordinates refer to the direction of motion of $\mathrm{S} 1$ ion in the Bi-S bi-layers at $\mathrm{z} \sim \frac{1}{3} c$ and $\mathrm{z} \sim \frac{2}{3} c$. Given that the lattice is nominally tetragonal, the a- and b-axes are equivalent. However, the magnitude of the displacement is not necessarily the same in both directions, and the direction, either -x- or -y-, of the displaced ion can be altered. The displacement direction at one layer is important relative to the second layer. [128]

Structure factor calculations were carried out for the $h k 0$ plane for the three main modes; $(+\mathrm{x},-\mathrm{x}),(+\mathrm{x},+\mathrm{x})$ and $(+\mathrm{x},+\mathrm{y})$ to which all the other sulfur modes are equivalent given the tetragonal symmetry. It is determined that when the displacement pattern is $(+\mathrm{x}$, -x), no intensity is produced at Miller index $h=0$ when $k$ is odd. When the displacement pattern is $(+\mathrm{x},+\mathrm{x})$, the intensity is zero again when either $h$ or $k$ become odd. Thus the $(+\mathrm{x},-\mathrm{x})$ and $(+\mathrm{x},+\mathrm{x})$ patterns are unlikely. Furthermore, the displacement patterns suggested in Ref. $[23,133]$ and $[134]$ that use the $P \mathscr{2}_{1} / m$ allows a $(+\mathrm{x},-\mathrm{x})$ S1 displacement pattern, where a Bragg structure with extra reflections as seen in Fig. 6.3(d) is produced. It is clear that $(+\mathrm{x},-\mathrm{x})$ fails to reproduce critical reflections at spots marked in the the $h k 0$ plane. Therefore, the only possibility for displacement is the $(+\mathrm{x},+\mathrm{y})$ mode.

The $(+\mathrm{x},+\mathrm{y})$ mode could be either ferro-distortive type or antiferro-distortive type. If the displacements are antiferro-distortive, the antiferro-distortive unit cell need to be doubled along the a- and b-axes as the mode itself requires the twice a size of unit cell. On the other hand, if the displacements are ferro-distortive, the ferro-distortive cell is 
not doubled. The ferro-distortive and antiferro-distortive sulfur modes are depicted in the schematic of Fig. 6.4.

(a)

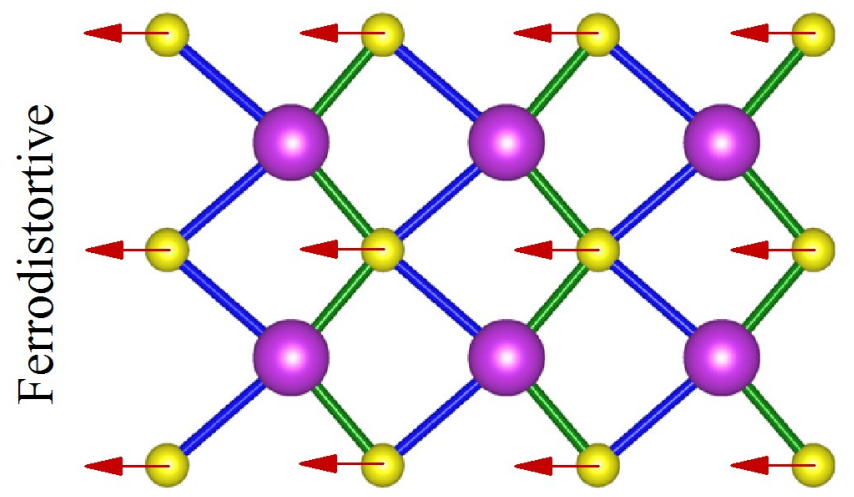

(b)

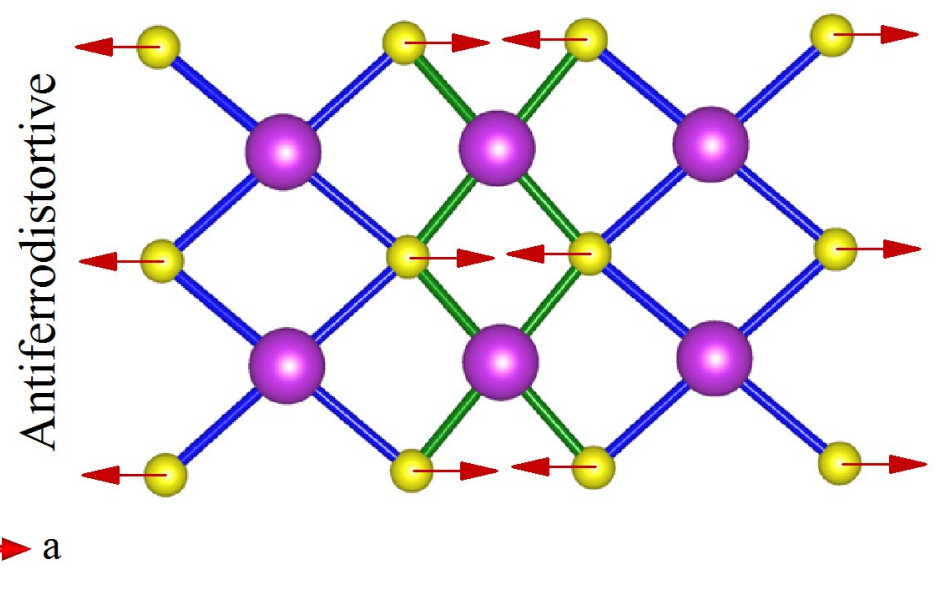

Figure 6.4: Displacement modes of sulfur. In (a) the ferro-distortive and (b) the antiferro-distortive displacement modes are shown.

The Bragg reflections in the $h k 0$ plane are calculated for the remaining displacement pattern $(+\mathrm{x},+\mathrm{y})$ (or equivalently $(-\mathrm{x},-\mathrm{y}))$ using the ferro- and antiferro-distortive modes in the $P 1$ symmetry. This displacement pattern breaks the fourfold, twofold and inversion symmetry operations of the nominal P4/nmm. In Fig. 6.5 (a) and (b), the calculated reciprocal lattice patterns corresponding to the ferro- and antiferro-distortive modes are shown, respectively. Far more reflections are observed in the antiferro-distortive mode of Fig. 6.5(b), and can readily be eliminated when compared to the data from $\mathrm{LaOBiS}_{2}$ (Fig. 6.1(a)) since it generates half integer peaks that are not actually present. On the 
other hand, the ferro-distortive mode can reproduce all Bragg spots observed. Thus the ferro-distortive mode of $(+\mathrm{x},+\mathrm{y})$ remains as the only likely possibility.

(a) Ferrodistortive

\begin{tabular}{|c|c|c|c|c|c|c|}
\hline 330 & $\begin{array}{c}230 \\
\bullet\end{array}$ & 130 & $\stackrel{030}{\odot}$ & 130 & $\stackrel{230}{\odot}$ & 330 \\
\hline $\begin{array}{c}320 \\
\bullet\end{array}$ & 220 & $\begin{array}{c}120 \\
\bullet\end{array}$ & 020 & $\stackrel{120}{\odot}$ & 220 & $\stackrel{320}{\odot}$ \\
\hline 310 & $\begin{array}{c}210 \\
\odot\end{array}$ & 110 & $\begin{array}{c}010 \\
\odot\end{array}$ & 110 & $\begin{array}{c}210 \\
\odot\end{array}$ & 310 \\
\hline $\begin{array}{c}300 \\
\odot\end{array}$ & 200 & $\begin{array}{l}100 \\
\odot\end{array}$ & & $\begin{array}{c}100 \\
\odot\end{array}$ & 200 & $\begin{array}{c}300 \\
\odot-\end{array}$ \\
\hline 310 & $\begin{array}{c}210 \\
\odot\end{array}$ & 110 & $\begin{array}{c}010 \\
\odot\end{array}$ & 110 & $\begin{array}{c}2 \overline{10} \\
\odot\end{array}$ & 310 \\
\hline$\stackrel{320}{\odot}$ & 220 & $\begin{array}{c}120 \\
\odot\end{array}$ & $0 \overline{2} 0$ & $\begin{array}{c}1 \overline{2} 0 \\
\bigodot\end{array}$ & $2 \overline{2} 0$ & $\begin{array}{c}3 \overline{2} 0 \\
\odot\end{array}$ \\
\hline 330 & $\stackrel{230}{\odot}$ & 130 & $\begin{array}{c}0 \overline{3} 0 \\
\odot\end{array}$ & $1 \overline{3} 0$ & $\stackrel{2 \overline{3} 0}{\odot}$ & $3 \overline{3} 0$ \\
\hline
\end{tabular}

(b) Antiferrodistortive

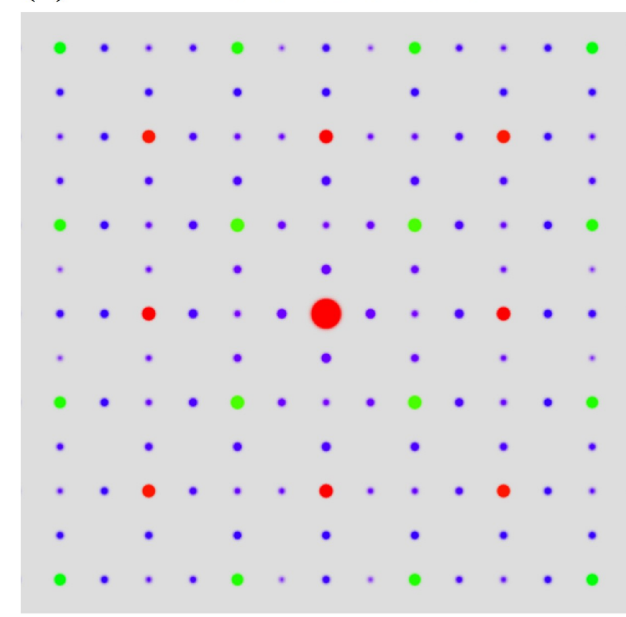

FiguRe 6.5: In (a) The calculated diffraction pattern from the ferro-distortive mode is shown while in (b) the diffraction pattern resulting from the antiferro-distortive mode is shown.

\subsubsection{Crystal structure refinement using $(+\mathrm{x},+\mathrm{y})$ displacement mode}

To reproduce the Bragg peak intensity, a refinement of the three dimensional neutron diffraction intensity is performed using $P 1$ symmetry. This involves a total of about 4600 Bragg peaks. The results from the refinement are listed in Table 6.6. The (x, y) distortion pattern shown in the top inset of Fig. 6.6 is considered with periodic boundary conditions. There are two $\mathrm{BiS}_{2}$ layers in the unit cell and the displacement vectors in the $\mathrm{x}$ or $\mathrm{y}$ direction are indicated with the arrows. The lattice or structural parameters do not deviate much from that of the nominal $P 4 / n m m$ symmetry except the in-plane sulfur position indicated with bold font in the Table 6.6, which simply breaks fourfold, twofold and inversion symmetry conditions of the nominal symmetry. The magnitude of 
TABLE 6.6: A list of crystal structure parameters for $\mathrm{LaOBiS}_{2}$ obtained from the neutron diffraction data refinement using ShelX. The P1 symmetry was used in the refinement.

\begin{tabular}{|c|c|c|c|c|}
\hline \multicolumn{5}{|c|}{$\mathrm{LaOBiS}_{2}$} \\
\hline & \multirow{3}{*}{\multicolumn{2}{|c|}{$\begin{array}{l}a(\AA)=4.0480(34) \\
b(\AA)=4.0514(13) \\
c(\AA)=13.8242(64)\end{array}$}} & \multirow{3}{*}{\multicolumn{2}{|c|}{$\begin{array}{l}\alpha=90.0147(323) \\
\beta=90.0356(547) \\
\gamma=90.0193(513)\end{array}$}} \\
\hline & & & & \\
\hline & & & & \\
\hline & $\mathbf{x}$ & $\mathbf{y}$ & $\mathbf{z}$ & $\mathbf{U}_{i s o}\left(\AA^{2}\right)$ \\
\hline La & $0.4953(71)$ & $-0.0036(5)$ & $0.0901(1)$ & $0.0091(3)$ \\
\hline La & $-0.0021(5)$ & $0.4937(4)$ & 0.9099(1) & $0.0091(3)$ \\
\hline $\mathrm{Bi}$ & $0.4911(9)$ & $0.0100(6)$ & $0.6305(1)$ & $0.0136(3)$ \\
\hline $\mathrm{Bi}$ & $-0.0006(8)$ & $0.5061(6)$ & $0.3695(1)$ & $0.0136(3)$ \\
\hline S1 & $0.4737(36)$ & $-0.0169(22)$ & $0.3841(4)$ & $0.0247(17)$ \\
\hline S1 & $0.0028(36)$ & $\mathbf{0 . 4 5 6 7}(22)$ & $0.6159(4)$ & $0.0247(17)$ \\
\hline $\mathrm{S} 2$ & $0.4938(20)$ & $0.0198(14)$ & $0.8105(3)$ & $0.0061(6)$ \\
\hline S2 & $0.0002(19)$ & $0.5122(14)$ & $0.1895(3)$ & $0.0061(6)$ \\
\hline $\mathrm{O}$ & $0.0149(11)$ & $0.0034(10)$ & $0.0002(4)$ & $0.0092(4)$ \\
\hline $\mathrm{O}$ & $0.5148(11)$ & $0.5027(10)$ & $0.0004(4)$ & $0.0092(4)$ \\
\hline
\end{tabular}

displacement in the two planes are different. Clearly, the thermal parameter, $\mathrm{U}_{i s o}$, of inplane sulfur S1 is also comparatively large. The calculated structure factor is compared to the observed structure function and the goodness of fit $R$ is 0.1148 . The fitting results are shown in Fig. 6.6. The plot between the observed and calculated squared intensities is on the expected line (blue line with a slope of 1 ), where the superlattice structure is well reproduced. Some differences are observed involving the highest intensity Bragg peaks. The in-plane sulfur distortions are 0.11 and $0.18 \AA$ in the two $\mathrm{BiS}_{2}$ planes and they are in the order of sulfur movements predicted by the state-of-the-art first principles calculations which is $0.101 \AA[15]$. It is clear that the sulfur ferro-displacement is a key component to the broken crystal symmetry. 


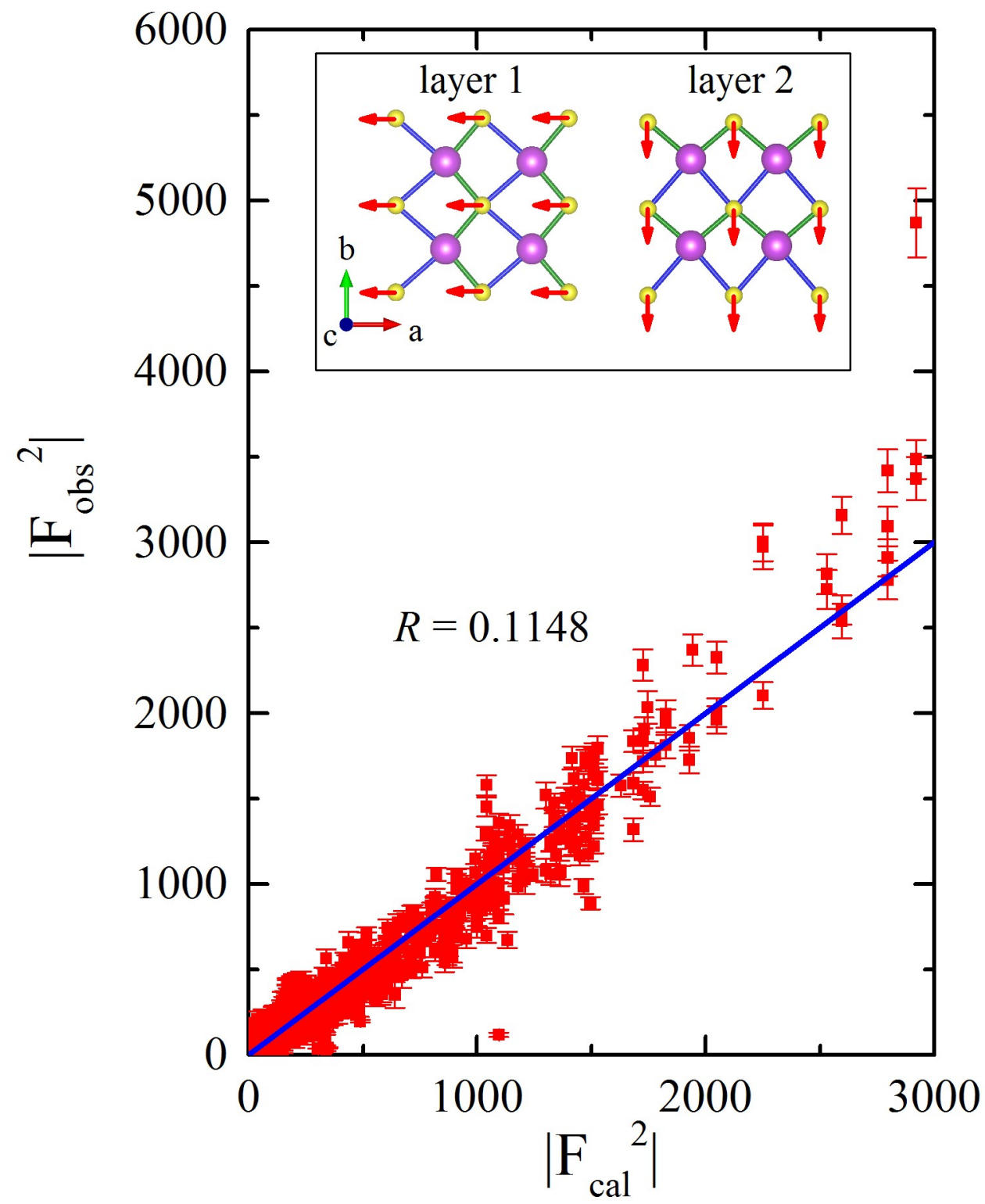

FiguRE 6.6: The single crystal refinement result. The square of the structure function, $|F(Q)|^{2}$, for the observed intensity is compared to the calculated intensity obtained from the refinement based on $(\mathrm{x}, \mathrm{y})$ mode. The fitting is done using the neutron single crystal data. The goodness of fit is determined to be 0.1148 . The blue line represents the ideal match between the calculated and the observed value at each Bragg peak with a slope of 1 . Deviations are observed for the highest intensity peaks. In the inset, the two $\mathrm{BiS}_{2}$ layers corresponding to displacements of $\mathrm{S} 1$ are shown. 


\subsubsection{Stacking of sulfur displacement modes as a candidate}

It is possible that if the crystal is twinned, the unit cell is rotated through 90 degrees about the c-axis, where the $(+\mathrm{x},+\mathrm{y})$ displacement pattern becomes $(+\mathrm{y},-\mathrm{x})$ and $(+\mathrm{x}$, -x) pattern becomes $(+\mathrm{y},-\mathrm{y})$. The combinations of $(+\mathrm{x},+\mathrm{y})$ and $(+\mathrm{y},-\mathrm{x})$ or $(+\mathrm{x},-\mathrm{x})$ and $(+\mathrm{y},-\mathrm{y})$ can generate all the $h+k=$ odd reflections just as in the case of single $(+\mathrm{x},+\mathrm{y})$ mode. This leaves us to question which pattern is more likely to occur, the (x,y), or a stacking of its equivalent modes, or a twinned $(+\mathrm{x},-\mathrm{x})$ ? The answer is in the pattern along the 00l-direction. The Bragg peaks along the 00l-direction are much broader than the $h k 0$-plane due to stacking faults as previously discussed [113]. However, a stacking pattern of an untwined $(\mathrm{x}, \mathrm{y})$ compared to its twined equivalents or a twinned $(+\mathrm{x},-\mathrm{x})$ mode will produce different diffraction patterns. If the $(\mathrm{x}, \mathrm{y})$ mode alternates along the c-axis with a (-x, -y) mode, extra reflections are created along $00 l$ as shown in Fig. 6.7. Similarly, if a twinned $(+\mathrm{x},-\mathrm{x})$ pattern is considered as proposed above with the $P \mathscr{2}_{1} / \mathrm{m}$ symmetry, we find that even if the rotation occurs every 50 unit cells along the c-axis, additional reflections are observed in $00 \mathrm{l}$ just like in the example of Fig. 6.7 that are not present (circled) in the data. Therefore, stacking of different modes is not a suitable candidate to account for the observed supperlattice Bragg peak pattern. Furthermore, the single $(+\mathrm{x},+\mathrm{y})$ mode or its equivalent modes individually considered remain as the only possibility to reproduce the observed diffraction pattern. 


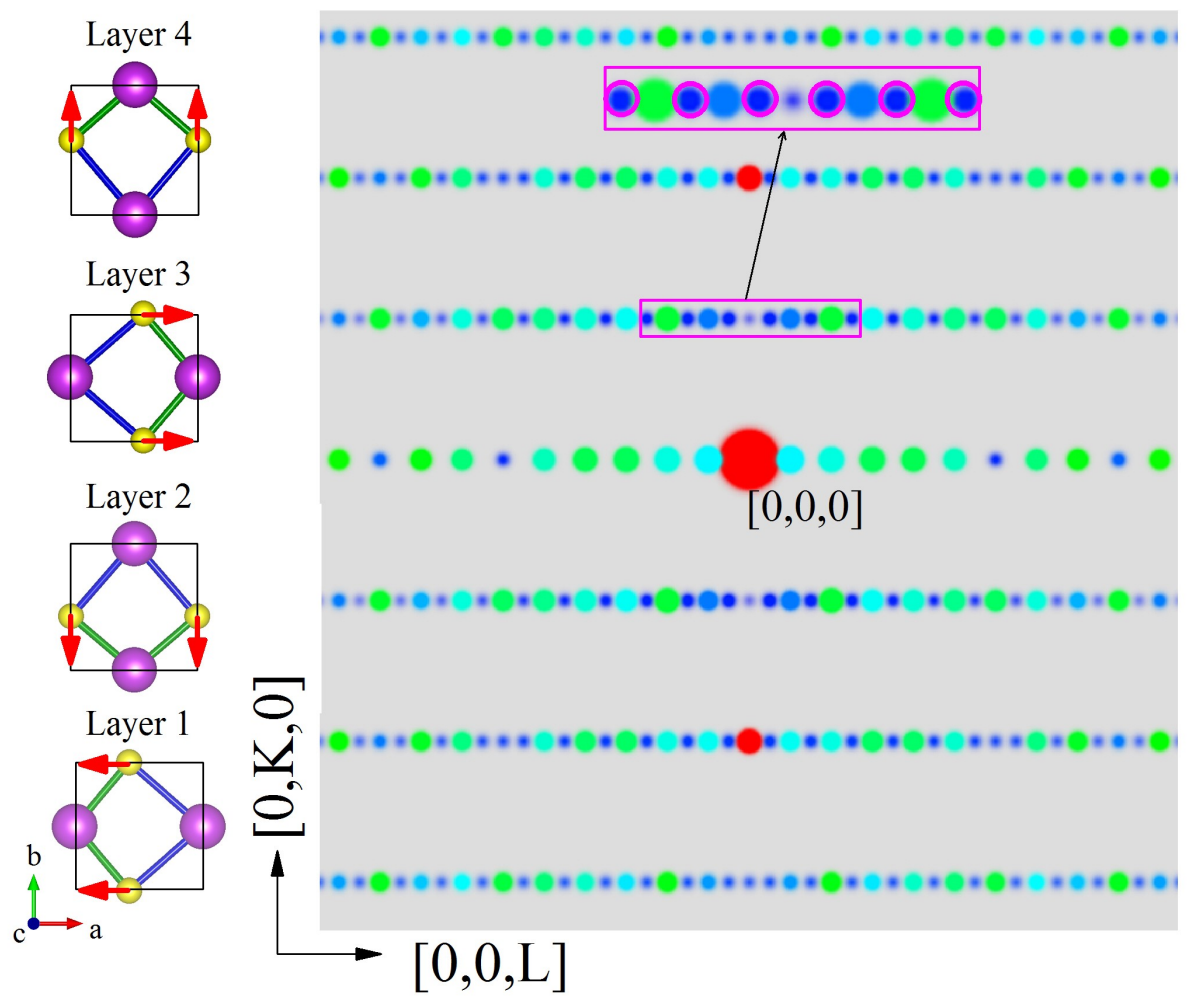

FiguRE 6.7: The calculated diffraction pattern is shown based on an alternating ( $\mathrm{x}$, y) mode with (-x, -y) mode along c-axis. The circled are the extra reflections not present in data. In the side panel, the four Bi-S planes in the doubled unit cell along $c$ depicting the alternating $(\mathrm{x}, \mathrm{y})$ mode and $(-\mathrm{x},-\mathrm{y})$ mode is shown.

\subsection{Diffraction patterns of $\mathrm{ReO}_{0.7} \mathbf{F}_{0.3} \mathrm{BiS}_{2}$}

The $(\mathrm{x}, \mathrm{y})$ model with the ferro-distortive cell could be used to reproduce the data collected from the $30 \%$ fluorine doped superconducting samples of $\mathrm{ReOBiS}_{2}(\mathrm{Re}=\mathrm{La}$, Nd and Pr). The single crystals are not exposed to high pressure annealing as in the case of powders hence their $\mathrm{T}_{c}$ 's are lower [25, 113] with $\sim 2.5 \mathrm{~K}$ and $\sim 5 \mathrm{~K}$ for $\operatorname{Re}$ $=\mathrm{La}$ and $\mathrm{Nd}$, respectively. The $\mathrm{F}$ doped samples are approximately ten times thinner 
(a) $\mathrm{LaOBiS}_{2}$

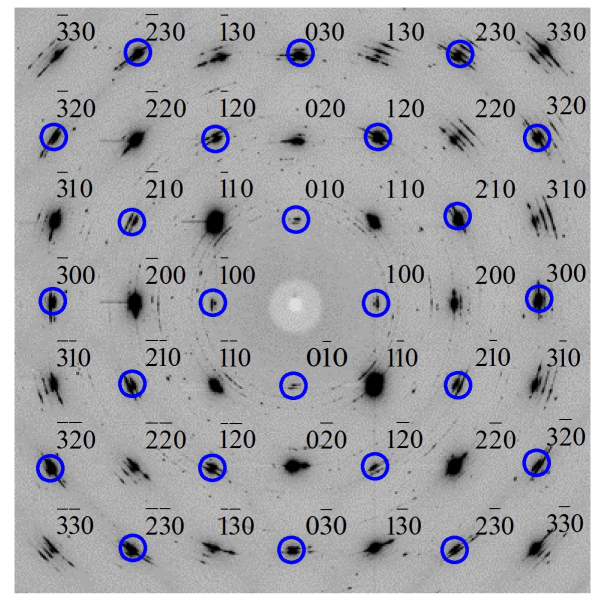

(c) $\mathrm{NdO}_{0.7} \mathrm{~F}_{0.3} \mathrm{BiS}_{2}$

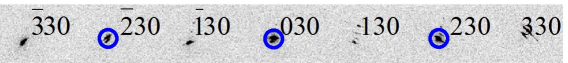

$$
\begin{aligned}
& \odot^{320} \cdot{ }^{220} \odot^{120} \cdot 020 \otimes^{120}:{ }^{220} \otimes^{320} \\
& \cdot^{310} 0^{210} e^{110} 0^{010} \cdot{ }^{110} \text { ब }^{210} 310 \\
& \odot^{300} \cdot i^{200} 0^{100} \quad 0^{100}, i^{200} \AA^{300}
\end{aligned}
$$

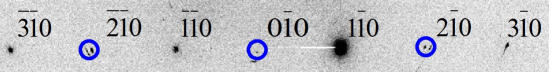

$$
\begin{aligned}
& \odot^{\overline{320}} \cdot{ }^{\overline{2} 20} \odot^{\overline{120}} \cdot 0^{\overline{2} 0} 0^{1 \overline{2} 0} \cdot{ }^{2 \overline{2} 0} \odot^{3 \overline{2} 0} \\
& { }^{\overline{3} 30} \odot^{\overline{23} 0} \cdot{ }^{\overline{13} 0} \odot^{0 \overline{3} 0} \cdot{ }^{\overline{1} 30} \odot^{2 \overline{3} 0} \cdot 3 \overline{3} 0
\end{aligned}
$$

(b) $\mathrm{LaO}_{0.7} \mathrm{~F}_{0.3} \mathrm{BiS}_{2}$

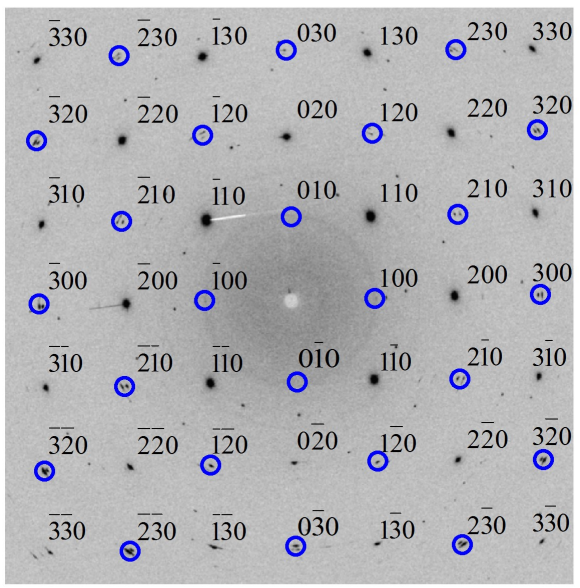

(d) $\mathrm{PrO}_{0.7} \mathrm{~F}_{0.3} \mathrm{BiS}_{2}$

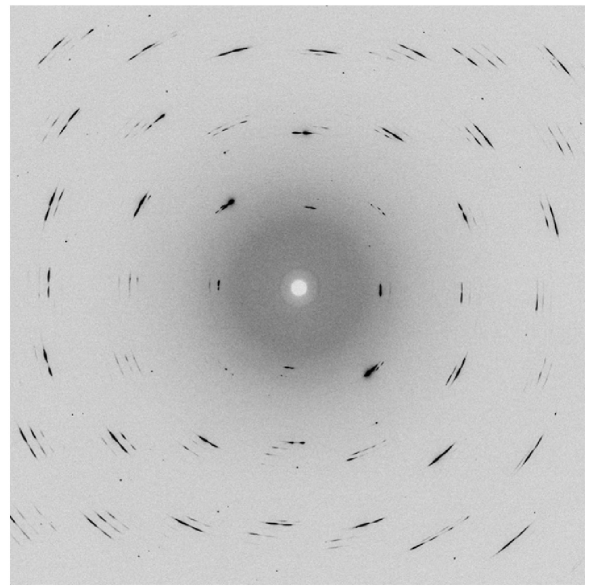

Figure 6.8: The synchrotron x-ray data in the $h k 0$ plane are shown for (a) $\mathrm{LaOBiS}_{2}$, (b) $\mathrm{LaO}_{0.7} \mathrm{~F}_{0.3} \mathrm{BiS}_{2}$, (c) $\mathrm{NdO}_{0.7} \mathrm{~F}_{0.3} \mathrm{BiS}_{2}$ and (d) $\mathrm{PrO}_{0.7} \mathrm{~F}_{0.3} \mathrm{BiS}_{2}$. The circled are the Bragg peaks when $h+k$ is odd ( $\mathrm{In} \mathrm{PrO}_{0.7} \mathrm{~F}_{0.3} \mathrm{BiS}_{2}, h+k$-odd peaks are not circled due to the poor crystallinity with powder streaks.).

compared to the parent sample, therefore, synchrotron x-ray is a more suitable tool than neutrons to probe the structure in this regard, due to the shorter penetration depth of x-rays before scattering and ability to focus finely. In Fig. 6.8 (a), (b), (c) and (d), the synchrotron x-ray diffraction patterns of $\mathrm{LaOBiS}_{2}, \mathrm{LaO}_{0.7} \mathrm{~F}_{0.3} \mathrm{BiS}_{2}, \mathrm{NdO}_{0.7} \mathrm{~F}_{0.3} \mathrm{BiS}_{2}$ and $\mathrm{PrO}_{0.7} \mathrm{~F}_{0.3} \mathrm{BiS}_{2}$ on the $h k 0$ plane are shown, respectively. The $h+k$-odd reflections (circled) can readily be seen just like in the parent compound, $\mathrm{LaOBiS}_{2}$. 


\subsection{Strain effect on $\mathrm{T}_{c}$ of $\mathrm{NdO}_{0.7} \mathrm{~F}_{0.3} \mathrm{BiS}_{2}$ single crystals}

The superconducting mechanism of $\mathrm{BiS}_{2}$ class is strongly correlated with lattice distortions and structural instabilities. As outlined in the previous sections of this thesis, sulfur displacements give rise to charge fluctuations around Bi that is most probably involved in the charge carrier mechanism. It is possible that changing macro level physical properties might have an impact on the nanoscale distortions of the system [135], thus a parameter such as strain might have an effect on the lattice distortions. These distortions may be coupled with the superconducting transition temperature $\mathrm{T}_{c}$ of the system. To investigate this possibility, we developed a in-situ tunable strain for $\mathrm{BiS}_{2}$ single crystal samples, and resistivity measurements were carried out using a standard 4-point configuration as a function of temperature.

Single crystal samples of $\mathrm{NdO}_{0.7} \mathrm{~F}_{0.3} \mathrm{BiS}_{2}$ have a plate-like formation with $c$-axis perpendicular to the plane of the plate. A single crystal sample and strain gauge were glued onto the side walls of the piezo stack using a two part epoxy resin. This step involved careful coating of epoxy on a single side of the crystal and gauge, gluing and baking at $80^{\circ} \mathrm{C}$ for 1 hour to gain a strong bond. The piezo stacks were cryogenic-coated by the manufacturer to withstand the low temperatures as 2 K. Strain was transferred to the single crystals by applying a positive voltage bias through the red and black voltage leads of the piezo stack which in response expands along the long dimension and contracts along the transverse direction. [135, 136] Fig. 6.9 shows a photograph of the set up that was used for the characterization of piezo stack before the experiment. The relative orientation of the crystal axes with respect to the piezo stack determines the direction in which the strain is applied. In this case the strain is applied along $a$ (or equivalently b) direction. The electrical contacts between the four probes of the resistivity option of 
the puck and the sample were made via platinum wires. The wires were connected to the four probes with $\mathrm{Sn} / \mathrm{Ag}$ solder and to the sample using two part silver paint which was baked at $80^{\circ} \mathrm{C}$ for 180 minutes to solidify.

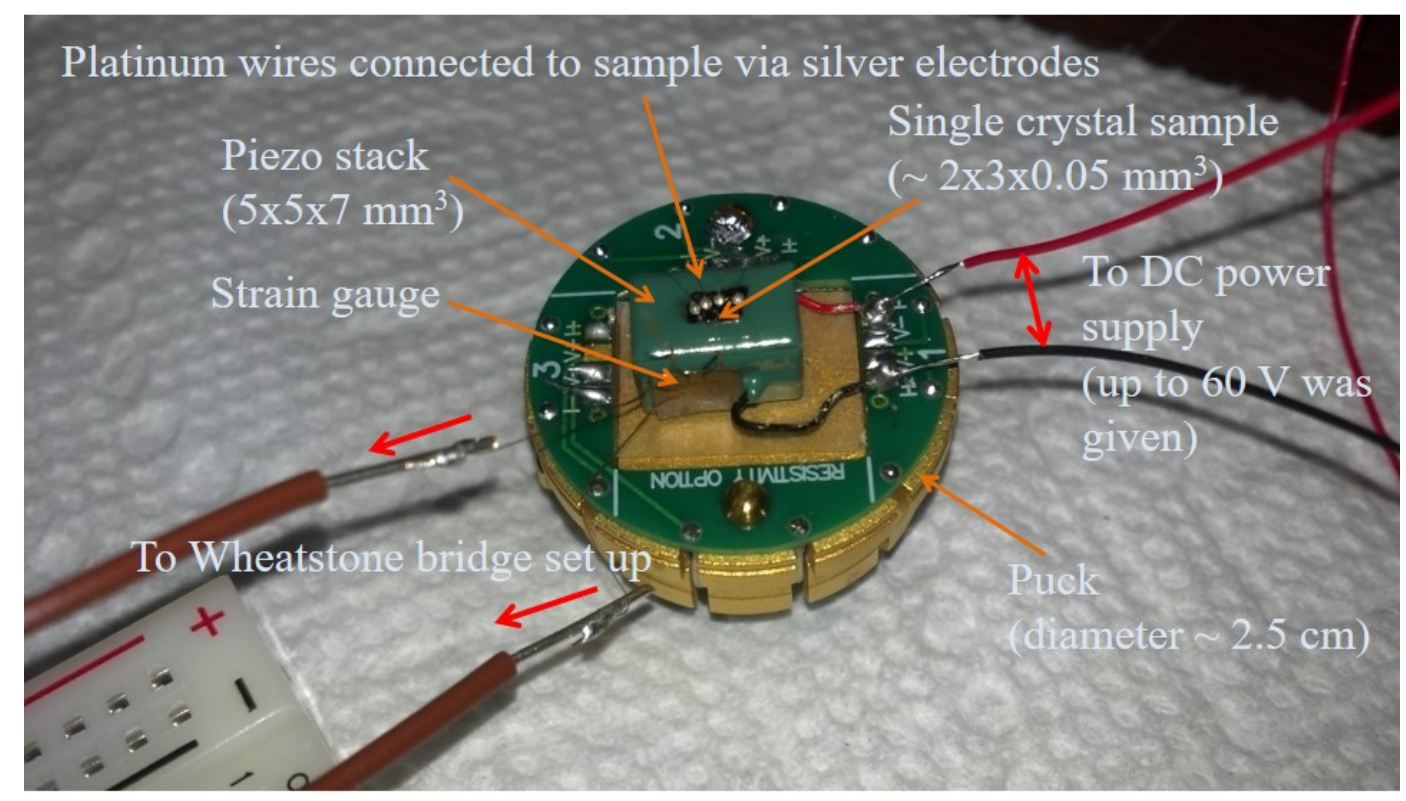

FIGURE 6.9: The photograph of the set up arranged for characterizing the piezo stack before measuring resistivity.

\subsubsection{Characterization of strain of piezo stack}

Strain is the amount of deformation of a body due to an applied force. More specifically, strain $(\varepsilon)$ is defined as the fractional change in length $\Delta L / L$, as shown in Fig. 6.10. In practice, the magnitude of measured strain involves quantities larger than a few millistrain. A strain gauge is a device whose electrical resistance varies in proportion to the amount of strain in the device. Measuring the strain requires accurate measurement of very small changes in resistance. Therefore a Wheatstone bridge (shown in Fig. 6.11) 
with four resistive arms was used to measure the small change in resistance of strain gauge.

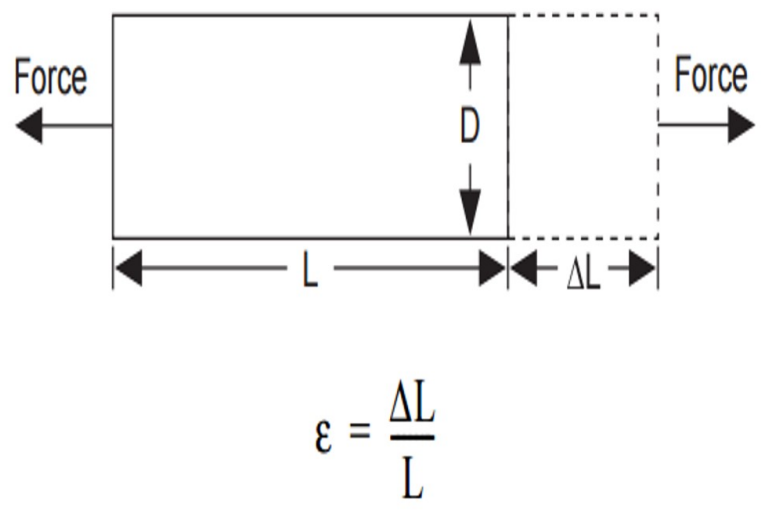

Figure 6.10: The definition of strain.

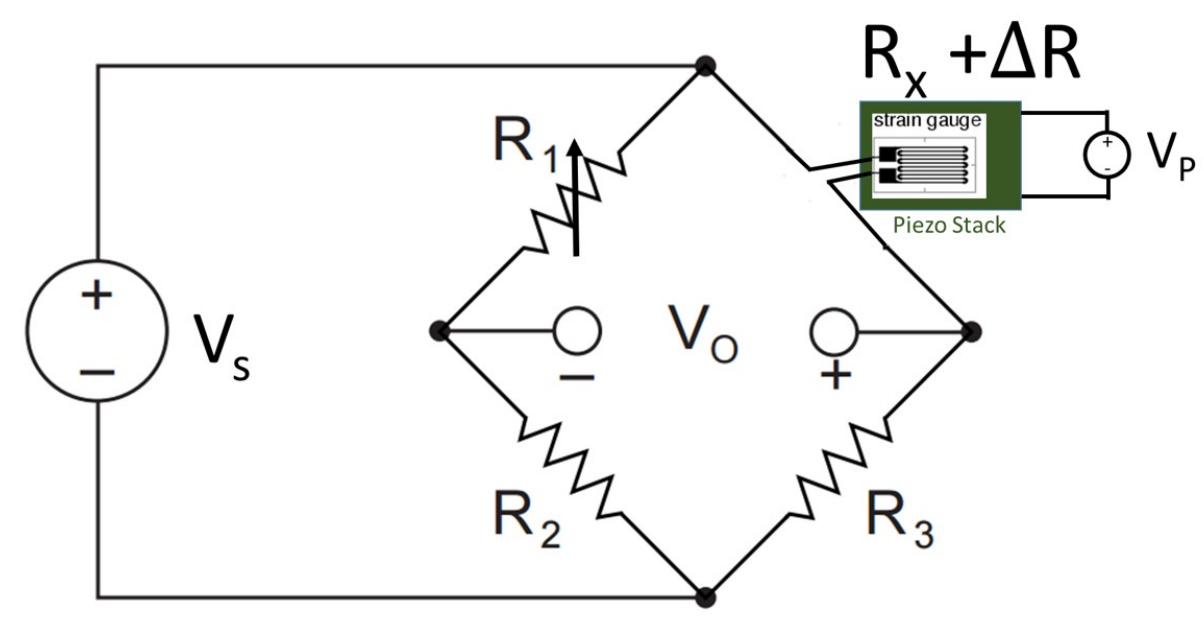

FIGURE 6.11: Wheatstone bridge with the strain gauge as one resistive arm.

A fundamental parameter of the strain gauge is its sensitivity to strain, expressed quantitatively as the gauge factor (GF). Gauge factor is defined as the ratio of fractional change in electrical resistance to the fractional change in length (strain), $\Delta L / L$ :

$$
G F=\frac{(\Delta R / R)}{(\Delta L / L)}=\frac{(\Delta R / R)}{\varepsilon}
$$


Gauge factor for the used strain gauge, $\mathrm{GF}=2.02$.

The bridge was balanced (make $V_{0}=0$ by adjusting the variable resister, $R 1$ ) when the applied voltage to piezo $\left(V_{p}\right)$ is zero. When a strain $(\varepsilon)$ occurs due to non-zero $V_{p}$, the bridge will result in a nonzero output voltage $V_{0}$.

$$
V_{0}=V_{S}\left(R_{3} /\left(R_{3}+\left(R_{x}+\Delta R\right)\right)-R_{2} /\left(R_{1}+R_{2}\right)\right)
$$

When the bridge was initially balanced,

$$
R_{1} / R_{2}=R_{x} / R_{3}
$$

and

$$
\Delta R=R_{x} G F \cdot \varepsilon
$$

Simplifying we can find an expression for strain,

$$
\varepsilon=\left(-V_{0}\left(R_{x}+R_{3}\right)^{2}\right) /\left(G F \cdot R_{x}\left(R_{3} V_{S}+V_{0}\left(R_{x}+R_{3}\right)\right)\right)
$$

The applied voltage to piezo was varied from $0 \mathrm{~V}$ to $60 \mathrm{~V}$ at room temperature and output voltage $V_{0}$ was measured. The resolution of voltmeter is $0.1 \mathrm{mV}$. The output voltage linearly increased (negatively) when applied piezo voltage was increased as shown in Fig. 6.12. Here the negative increase corresponds to an expansion.

The strain corresponding to the output voltage was calculated and graphed as in Fig. 6.13 as a function of applied voltage on piezo. The strain changes linearly with applied 


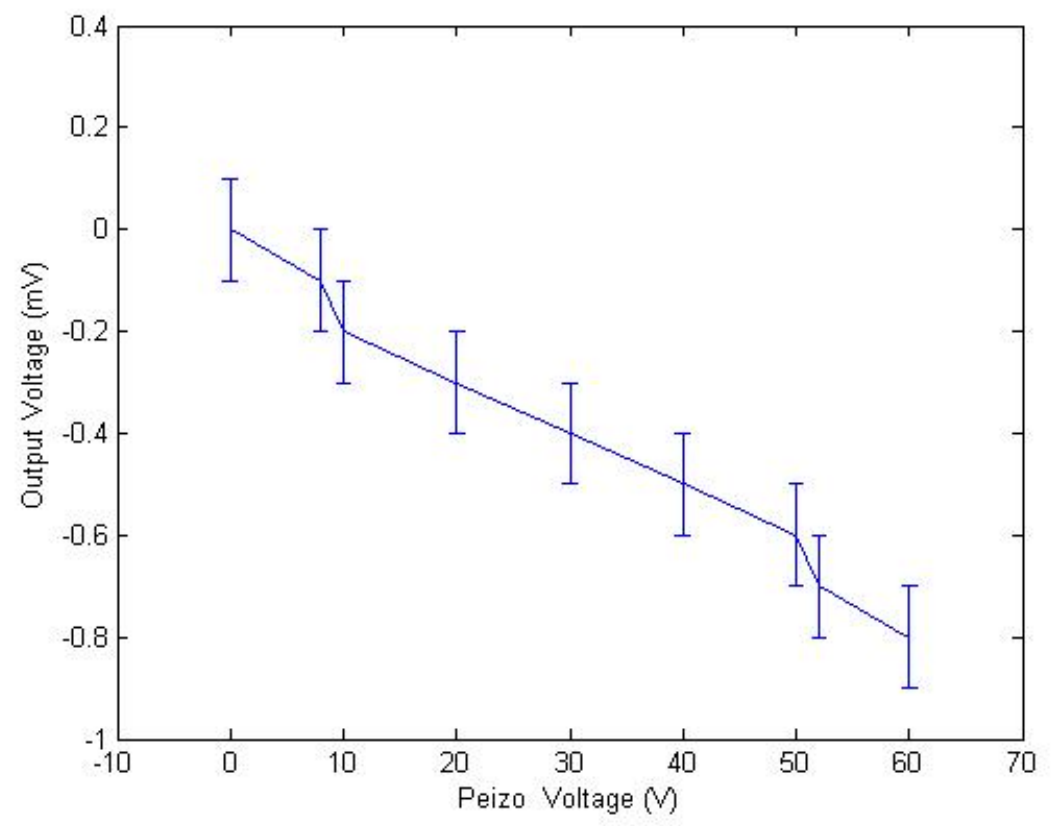

Figure 6.12: Output voltage of the bridge vs applied piezo voltage is shown where the output voltage, $V_{0}$ linearly increased negatively indicating an expansion.

voltage on piezo. Fitting the data gives $\varepsilon=11(1) \times 10^{-4}$ at $V_{p}=150 \mathrm{~V}$. The strain is in the range the manufacturer had specified, which was $\varepsilon=10 \times 10^{-4}$ at $V_{p}=150 \mathrm{~V}$. Therefore, we can conclude that the specific piezo stack in use was working and the strain was transferred to the gauge effectively via the glue in between the piezo and gauge. From the information obtained by the manufacturer, the strain at low temperatures as $2 \mathrm{~K}$ might be ten times less than that of at room temperature, therefore the effective strain applied on the sample around its $\mathrm{T}_{c}$ is much lower. 


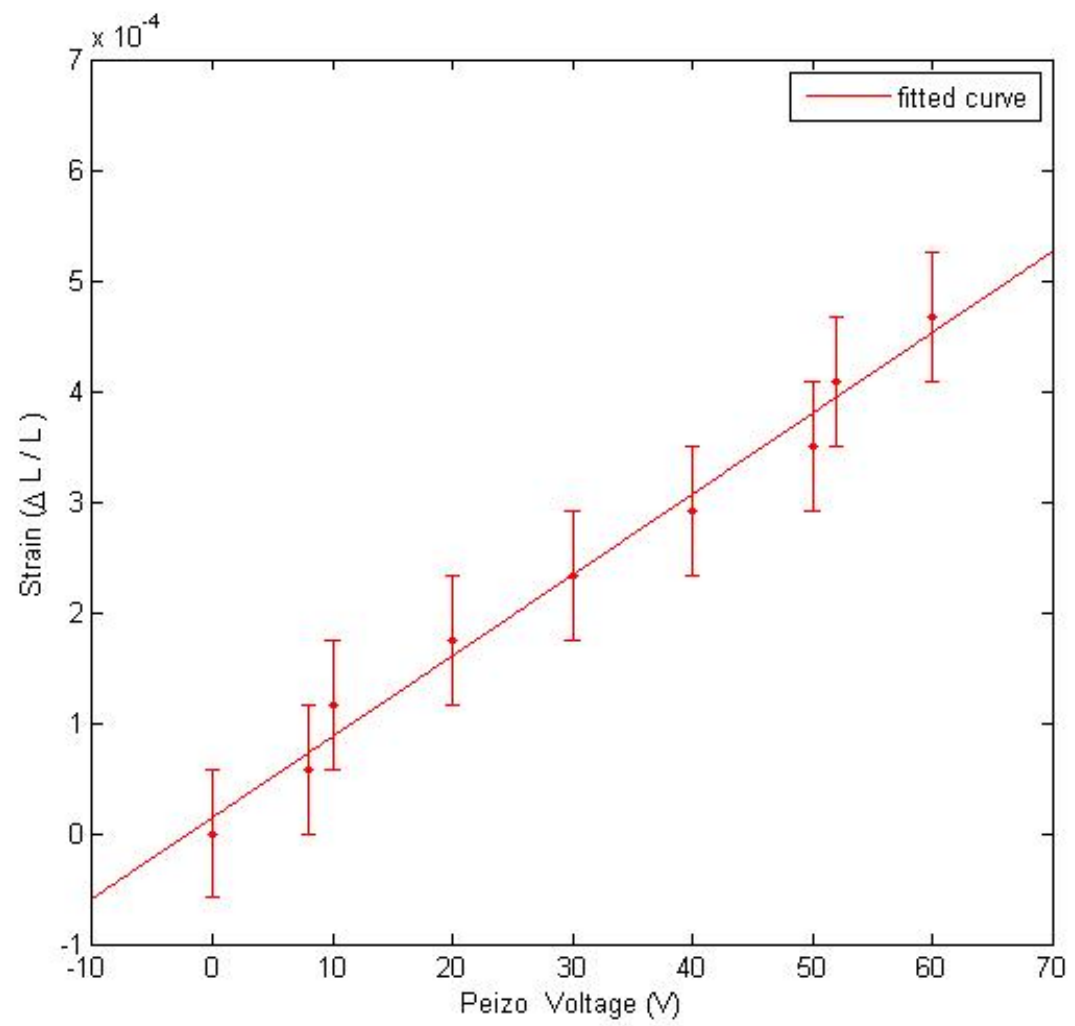

FiguRE 6.13: Strain vs.applied piezo voltage at room temperature.

\subsubsection{Resistance of $\mathrm{NdO}_{0.7} \mathrm{~F}_{0.3} \mathrm{BiS}_{2}$ single crystals under strain}

The resistance of $\mathrm{NdO}_{0.7} \mathrm{~F}_{0.3} \mathrm{BiS}_{2}$ was measured along the strain direction as a function of temperature using the physical property measuring system (PPMS). The strain was applied with a bias voltage on piezo of $49 \mathrm{~V}$ as it was the highest safest voltage recommended, inside the PPMS. Resistance was measured using the standard four probe technique while temperature was varied between 2 and $300 \mathrm{~K}$ for two cooling and warming cycles; with $0 \mathrm{~V}$ (no strain) and $49 \mathrm{~V}$ (with strain). The resistance plot as a function of temperature is shown in Fig. 6.14. There was no difference in either superconductivity onset temperature $\left(\mathrm{T}_{c 1}\right)$ or bulk superconducting temperature $\left(\mathrm{T}_{c 2}\right)$ between the two cases of $0 \mathrm{~V}$ and $49 \mathrm{~V}$ bias voltages. Therefore, we conclude that a strain with 49 $\mathrm{V}$ bias voltage at low temperatures is not sufficient to change the $\mathrm{T}_{c}$ of $\mathrm{NdO}_{0.7} \mathrm{~F}_{0.3} \mathrm{BiS}_{2}$ 
single crystals.

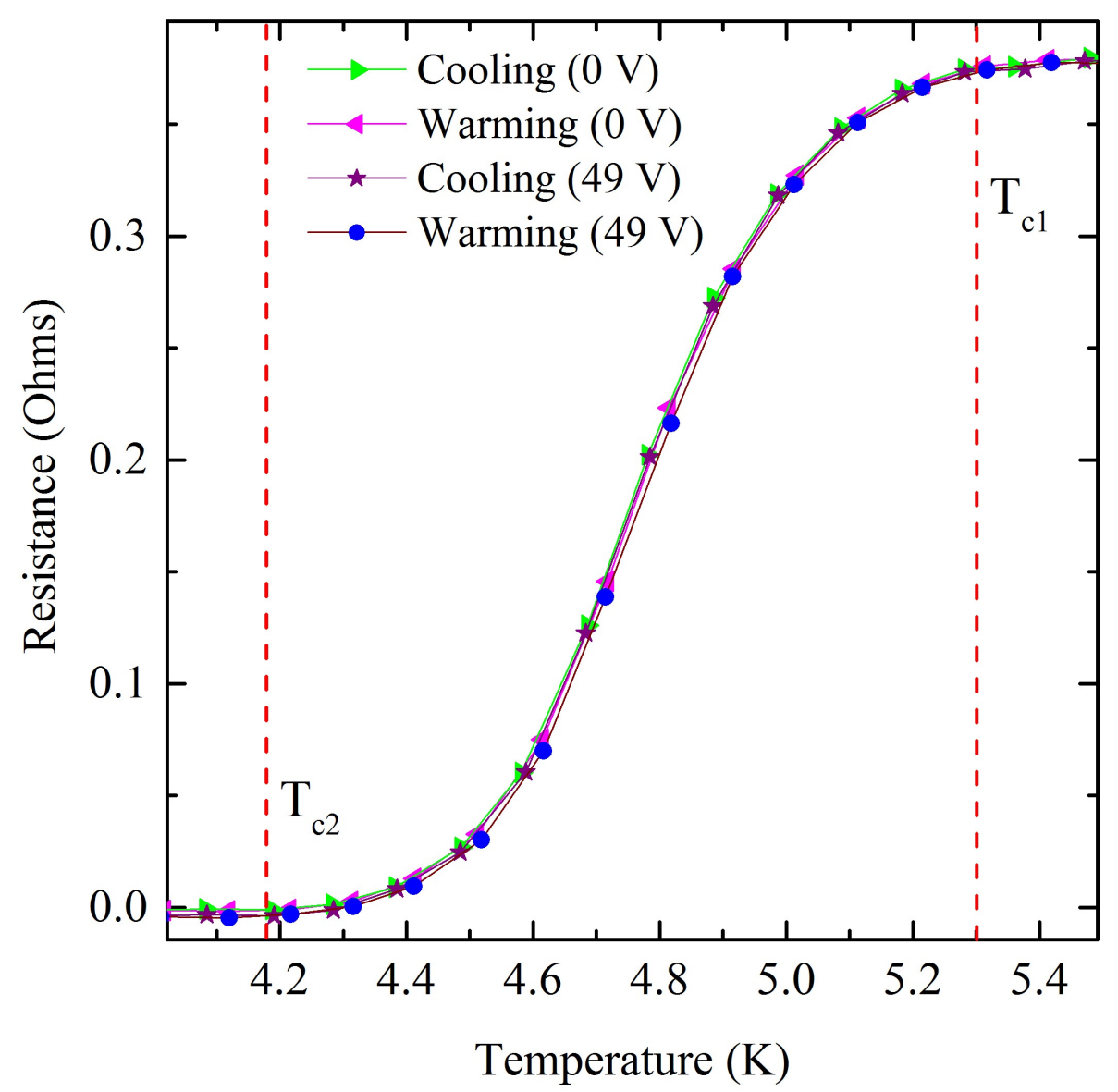

FiguRE 6.14: Resistance of $\mathrm{NdO}_{0.7} \mathrm{~F}_{0.3} \mathrm{BiS}_{2}$ single crystals as a function of temperature, without strain $(0 \mathrm{~V})$ and with strain $(49 \mathrm{~V})$. 


\subsection{Summary}

The structure of $\mathrm{ReO}_{1-x} \mathrm{~F}_{x} \mathrm{BiS}_{2}(\mathrm{Re}=\mathrm{La}, \mathrm{Nd}$ and $\mathrm{Pr})$ is exposed using neutron and synchrotron x-ray diffraction experiments. The sulfur mode that is responsible for the structural properties of $\mathrm{LaOBiS}_{2}$ is ferro-distortive in nature, where the in-plane displacement pattern can explain the single crystal results and must be responsible for the large electron-phonon coupling.[137] The Bi-S1 bond lengths are split in the parent compound, attesting to possible charge fluctuations and disproportionation. The sulfur displacements observed can explain the Bragg peak patterns of the superconducting crystals as well. Fluctuations of the bond lengths in the superconducting crystals indicate that charge fluctuations persist and may be significant in the mechanism of superconductivity. At low temperatures, no effect from applied strain was observed in $\mathrm{T}_{c}$ up to a piezo bias voltage of $49 \mathrm{~V}$ which is equal to a strain of $\sim 4 \times 10^{-5}$. 


\section{Chapter 7}

\section{Conclusions}

In phonon mediated superconductors, resolving the essential features of lattice modes that become active in connection with the superconducting transition might be key to understanding the mechanism. The crystal lattice of the $\mathrm{BiS}_{2}$ based superconductors plays an important role in superconductivity because of its potential to exhibiting a strong structure-property relationship due to its intrinsic disorder. The $\mathrm{ReO}_{1-x} \mathrm{~F}_{x} \mathrm{BiS}_{2}$ $(\mathrm{Re}=\mathrm{La}, \mathrm{Nd}$ and $\mathrm{Pr})$ system was investigated using neutron and synchrotron $\mathrm{x}$-ray diffraction to reveal the nature of electron-phonon coupling that leads to unconventional states with intricate microstructures and physical properties.

In $\mathrm{LaO}_{1-x} \mathrm{~F}_{x} \mathrm{BiS}_{2}$, the central issue to the coupling mechanism is an electron phonon lattice instability that potentially fosters competing ferroelectric and charge density wave states as suggested by first principles calculations. In this regard, the current study on polycrystalline samples confirmed that a long range structural order exists as evidenced from the Bragg structure, however, disorder is strong in the high pressure annealed superconducting sample with large distortions of $\mathrm{BiS}_{2}$ planes. The distortion of $\mathrm{BiS}_{2}$ plane remains at all doping levels, however, differences are observed in magnitude. Theoretically, it was expected that the distortion will vanish at the superconductivity phase, 
however, experimental results prove that distortions are also involved in the superconducting phase. Furthermore, the lower Debye temperature of the superconducting $x=0.5$ sample compared to its parent composition indicated a softer superconducting lattice which may allow enhanced lattice vibrations.

Local lattice distortions in both superconducting and non-superconducting $\mathrm{LaO}_{1-x} \mathrm{~F}_{x} \mathrm{BiS}_{2}$ compositions are manifested with in-plane sulfur distortions which give rise to split of Bi-S bonds. The splitting gives rise to two different bond lengths around $\mathrm{Bi}$ atoms and the bond length formations propose two types of displacement modes for sulfur; ferro-distortive type or antiferro-distortive type. The bond lengths in the two types of modes are equal in magnitude. The two proposed modes create unequal charge distributions around $\mathrm{Bi}$ atom and thus give rise to local charge fluctuations. Compared to the parent compound, the magnitude of sulfur displacement is reduced in the high pressure annealed $x=0.5$ superconducting samples while the out-of plane z-motion of apical sulfur increases. This is reminiscent of another phonon superconductor, $\mathrm{BaBiO}_{3}$, of which the Bi-O bonds are split at 2.11 and $2.29 \AA$ [138] due to a breathing [139] or a Peierls-like[140] instability where the strong coupling of the conduction electrons to the O phonons could explain the enhanced $\mathrm{T}_{c}$. However, with $\mathrm{K}$ doping as in $\mathrm{Ba}_{0.6} \mathrm{~K}_{0.4} \mathrm{BiO}_{3}$, the split disappears and the bond lengths become one, $2.14 \AA[138,141]$, which is not the case observed in the $\mathrm{LaO}_{1-x} \mathrm{~F}_{x} \mathrm{BiS}_{2}$ superconductor in which the distortions are present in the superconducting phase, albeit reduced in magnitude. Furthermore, even though $\mathrm{LaO}_{1-x} \mathrm{~F}_{x} \mathrm{BiS}_{2}$ is not magnetic, theoretical calculations showed that spin orbit coupling leads to a hidden spin polarization that has a considerable effect on the electronic band structure around the X-point of the Brillouin zone [33]. How the proposed lattice modes in $\mathrm{LaO}_{1-x} \mathrm{~F}_{x} \mathrm{BiS}_{2}$ affect this spin polarization will be interesting to investigate. 
At the optimum doping level, $\mathrm{NdO}_{1-x} \mathrm{~F}_{x} \mathrm{BiS}_{2}$ shows a comparatively higher transition temperature than $\mathrm{LaO}_{1-x} \mathrm{~F}_{x} \mathrm{BiS}_{2}$ without high pressure annealing. The polycrystalline samples have a higher chemical pressure in the $\mathrm{NdO}_{1-x} \mathrm{~F}_{x} \mathrm{BiS}_{2}$ unit cell compared to $\mathrm{LaO}_{1-x} \mathrm{~F}_{x} \mathrm{BiS}_{2}$ asserted by the significantly reduced unit cell volume. Both nonsuperconducting $x=0.2$ and superconducting $x=0.4$ lattices are locally distorted driven by atomic displacements. At the lower doping, the in-plane atomic distortions involve sulfur while at the higher doping both sulfur and bismuth contribute to the distortion. These atomic distortions are followed by asymmetric bond length formations around Bi which lead to charge fluctuations. The asymmetric coordination around Bi is reminiscent of the environment of $\mathrm{Bi}$ in $\mathrm{Cu}_{4} \mathrm{Bi}_{5} \mathrm{~S}_{10}$ due to the presence of a lone pair. [142] Similarly to the superconducting $\mathrm{LaO}_{1-x} \mathrm{~F}_{x} \mathrm{BiS}_{2}$, the superconducting phase of $\mathrm{NdO}_{1-x} \mathrm{~F}_{x} \mathrm{BiS}_{2}$ manifests z-motion of apical sulfur which could act as a charge transfer conduit between the electron doping layers and superconducting layers.

Several investigations were carried out regarding the precise structure of $\mathrm{ReO}_{1-x} \mathrm{~F}_{x} \mathrm{BiS}_{2}$ $(\mathrm{Re}=\mathrm{La}, \mathrm{Nd}$ and $\mathrm{Pr})$ and different symmetries were theoretically suggested based on lattice instability that gave rise to unstable phonon modes and charge density waves, and hidden spin polarization driven by atomic site asymmetry. In the current project, evidence is presented from single crystal synchrotron X-ray and neutron diffraction that challenges all theoretically suggested symmetries and true lattice symmetry is revealed. The observed Bragg structure from the $\mathrm{BiS}_{2}$ planes arises from $\mathrm{x}-\mathrm{y}$ displacements of sulfur that morph into coordinated ferro-distortive patterns in the $a b$-plane. This mode must bring significant changes in the electronic structure around the Fermi level. The pattern remains with electron doping and the fluctuations of the bond lengths in the superconducting crystal as shown here indicate that charge fluctuations persist and 
are most likely significant in the mechanism of superconductivity. Thus the electronic pairing in superconductivity present in this system is sensitive to the precise nature of the crystal structure.

Superconductivity in the presence of strong structural disorder might be a play field for discovering novel superconducting states, and $\mathrm{BiS}_{2}$ superconductors is a potential candidate. Charge fluctuations are present in the superconducting plane of all the $\mathrm{ReO}_{1-x} \mathrm{~F}_{x} \mathrm{BiS}_{2}(\mathrm{Re}=\mathrm{La}, \mathrm{Nd}$ and $\mathrm{Pr})$ compositions, as observed indirectly by their effects on the lattice. The distortions around Bi atoms are similar in nature in all the compositions, with coordinated ferrodistortive type modes. The charge fluctuations on the superconducting plane play an important role in the vicinity of superconductivity and can be coupled to the donor planes via z-motion of apical sulfur which could act as the charge transfer conduit in the pairing mediation of $\mathrm{BiS}_{2}$ superconductors. 


\section{Bibliography}

[1] H. K. Onnes. The resistance of pure mercury at helium temperatures. Commun. Phys. Lab. Univ. Leiden., 12:120, 1911.

[2] H. G. Smith and J. O. Wilhelm. Superconductivity. Rev. Mod. Phys., 7:237, 1935.

[3] H. H. Hill and B. T. Matthias. Study of the superconductivity of the intermetallic compounds $\mathrm{U}_{6} \mathrm{Mn}, \mathrm{U}_{6} \mathrm{Fe}, \mathrm{U}_{6} \mathrm{Co}$, and $\mathrm{U}_{6} \mathrm{Ni}$ and alloys formed between them. Phys. Rev., 168:464, 1968.

[4] A. Mohammad and D. A. Jyoti. Superconductivity in organic materials: a fascinating phenomenon. Research Journal of Chemical Sciences, 2(11):67, 2012.

[5] T. He, Q. Huang, A. P. Ramirez, Y. Wang, K. A. Regan, N. Rogado, M. A. Hayward, M. K. Haas, J. S. Slusky, K. Inumara, H. W. Zandbergen, N. P. Ong, and R. J. Cava. Superconductivity in the non-oxide perovskite $\mathrm{MgCNi}_{3}$. Nature, 411:54, 2001.

[6] S. L. Kakani K. P. Sinha. High temperature superconductivity: current results and novel mechanisms. Nova Science Publishers, 1994. ISBN 1-56072-133-2.

[7] S. Blundell. Superconductivity: a very short introduction. Oxford University Press, 2009. ISBN 978-0-19-954090-7.

[8] Y. Mizuguchi, H. Fujihisa, Y. Gotoh, K. Suzuki, H. Usui, K. Kuroki, S. Demura, Y. Takano, H. Izawa, and O. Miura. $\mathrm{BiS}_{2}$-based layered superconductor $\mathrm{Bi}_{4} \mathrm{O}_{4} \mathrm{~S}_{3}$. Phys. Rev. B, 86:220510, 2012.

[9] W. Meissner and R. Ochsenfeld. Ein neuer effekt bei eintritt der supraleitfähigkeit. Naturwissenschaften, 21(44):787, 1933.

[10] L. N. Cooper. Bound electron pairs in a degenerate Fermi gas. Phys. Rev., 104: 1189, 1956.

[11] J. Bardeen, L. N. Cooper, and J. R. Schrieffer. Microscopic theory of superconductivity. Phys. Rev., 106:162, 1957. 
[12] J. Bardeen, L. N. Cooper, and J. R. Schrieffer. Theory of superconductivity. Phys. Rev., 108:1175, 1957.

[13] G. Burns. High temperature superconductivity: an introduction. Academic Press Inc., 1992. ISBN 0-12-146090-8.

[14] P. Morel and P. W. Anderson. Calculation of the superconducting state parameters with retarded electron-phonon interaction. Phys. Rev., 125(4):1263, 1962.

[15] T. Yildirim. Ferroelectric soft phonons, charge density wave instability, and strong electron-phonon coupling in $\mathrm{BiS}_{2}$ layered superconductors: a first-principles study. Phys. Rev. B, 87:020506, 2013.

[16] Y. Feng, H-C. Ding, Y. Du, X. Wan, B. Wang, S. Y. Savrasov, and C-G. Duan. Electron-phonon superconductivity in $\mathrm{LaO}_{0.5} \mathrm{~F}_{0.5} \mathrm{BiSe}_{2}$. Journal of Applied Physics, 115(23):233901, 2014.

[17] X. Wan, H-C. Ding, S. Y. Savrasov, and C-G. Duan. Electron-phonon superconductivity near charge-density-wave instability in $\mathrm{LaO}_{0.5} \mathrm{~F}_{0.5} \mathrm{BiS}_{2}$ : Densityfunctional calculations. Phys. Rev. B, 87:115124, 2013.

[18] B. Li, Z. W. Xing, and G. Q. Huang. Evolution of superconductivity in $\mathrm{LaO}_{1-x} \mathrm{~F}_{x} \mathrm{BiS}_{2}$ prepared by high-pressure technique. Europhysics Letters, 101: 47002, 2013.

[19] P. S. Shruti and S. Patnaik. Evidence for fully gapped strong coupling s-wave superconductivity in $\mathrm{Bi}_{4} \mathrm{O}_{4} \mathrm{~S}_{3}$. Journal of Physics: Condensed Matter, 25(31): $312202,2013$.

[20] G. Lamura, T. Shiroka, P. Bonfà, S. Sanna, R. De Renzi, C. Baines, H. Luetkens, J. Kajitani, Y. Mizuguchi, O. Miura, K. Deguchi, S. Demura, Y. Takano, and M. Putti. s-wave pairing in the optimally doped $\mathrm{LaO}_{0.5} \mathrm{~F}_{0.5} \mathrm{BiS}_{2}$ superconductor. Phys. Rev. B, 88:180509, 2013.

[21] Z. R. Ye, H. F. Yang, D. W. Shen, J. Jiang, X. H. Niu, D. L. Feng, Y. P. Du, X. G. Wan, J. Z. Liu, X. Y. Zhu, H. H. Wen, and M. H. Jiang. Electronic structure of single-crystalline $\mathrm{NdO}_{0.5} \mathrm{~F}_{0.5} \mathrm{BiS}_{2}$ studied by angle-resolved photoemission spectroscopy. Phys. Rev. B, 90:045116, 2014.

[22] L. Sheng, Y. Huan, F. DeLong, W. ZhenYu, T. Jian, D. XiaXin, and W. HaiHu. Strong coupling superconductivity and prominent superconducting fluctuations in the new superconductor $\mathrm{Bi}_{4} \mathrm{O}_{4} \mathrm{~S}_{3}$. Sci. China - Phys. Mech. Astron., 56(11):2019, 2013. 
[23] J. Liu, D. Fang, Z. Wang, J. Xing, Z. Du, S. Li, X. Zhu, H. Yang, and H-H. Wen. Giant superconducting fluctuation and anomalous semiconducting normal state in $\mathrm{NdO}_{1-x} \mathrm{~F}_{x} \mathrm{Bi}_{1-y} \mathrm{~S}_{2}$ single crystals. EPL, 106:67002, 2014.

[24] G. B. Martins, A. Moreo, and E. Dagotto. RPA analysis of a two-orbital model for the $\mathrm{BiS}_{2}$-based superconductors. Phys. Rev. B, 87:081102, 2013.

[25] Y. Mizuguchi, S. Demura, K. Deguchi, Y. Takano, H. Fujihisa, Y. Gotoh, H. Izawa, and O. Miura. Superconductivity in novel $\mathrm{BiS}_{2}$-based layered superconductor $\mathrm{LaO}_{1-x} \mathrm{~F}_{x} \mathrm{BiS}_{2}$. J Phys. Soc. Jpn., 81:114725, 2012.

[26] D. Yazici, K. Huang, B. D. White, A.H. Chang, A. J. Friedman, and M.B. Maple. Superconductivity of F-substituted $\mathrm{LnOBiS}_{2}(\mathrm{Ln}=\mathrm{La}, \mathrm{Ce}, \mathrm{Pr}, \mathrm{Nd}, \mathrm{Yb})$ compounds. Philosophical Magazine, 93(6):673, 2013.

[27] D. Yazici, K. Huang, B. D. White, I. Jeon, V. W. Burnett, A. J. Friedman, I. K. Lum, M. Nallaiyan, S. Spagna, and M. B. Maple. Superconductivity induced by electron doping in $\mathrm{La}_{1-x} \mathrm{M}_{x} \mathrm{OBiS}_{2}(M=\mathrm{Ti}, \mathrm{Zr}, \mathrm{Hf}$, Th). Phys. Rev. B, 87:174512, 2013.

[28] X. Lin, X. Ni, B. Chen, X. Xu, X. Yang, J. Dai, Y. Li, X. Yang, Y. Luo, Q. Tao, G. Cao, and Z. Xu. Superconductivity induced by La doping in $\mathrm{Sr}_{1-x} \mathrm{La}_{x} \mathrm{FBiS}_{2}$. Phys. Rev. B, 87:020504, 2013.

[29] R. Jha, B. Tiwari, and V. P. S. Awana. Appearance of bulk superconductivity under hydrostatic pressure in $\mathrm{Sr}_{0.5} \mathrm{RE}_{0.5} \mathrm{FBiS}_{2}$ (re=ce, nd, pr, and sm) compounds. Journal of Applied Physics, 117(1):013901, 2015.

[30] H-F. Zhai, Z-T. Tang, H. Jiang, K. Xu, K. Zhang, P. Zhang, J-K. Bao, Y-L. Sun, W-H. Jiao, I. Nowik, I. Felner, Y-K. Li, X-F. Xu, Q. Tao, C-M. Feng, Z-A. Xu, and G-H. Cao. Possible charge-density wave, superconductivity, and f-electron valence instability in $\mathrm{EuBiS}_{2}$ F. Phys. Rev. B, 90:064518, 2014.

[31] K. Suzuki, M. Tanaka, S. J. Denholme, M. Fujioka, T. Yamaguchi, H. Takeya, and Y. Takano. Pressure-induced superconductivity in $\mathrm{BiS}_{2}$-based $\mathrm{EuFBiS}_{2}$. Journal of the Physical Society of Japan, 84(11):115003, 2015.

[32] Y. Mizuguchi, E. Paris, T. Wakita, G. Jinno, A. Puri, K. Terashima, B. Joseph, O. Miura, T. Yokoya, and N. L. Saini. Evolution of Eu valence and superconductivity in layered $\mathrm{Eu}_{0.5} \mathrm{La}_{0.5} \mathrm{FBiS}_{2-x} \mathrm{Se}_{x}$ system. Phys. Rev. B, 95:064515, 2017.

[33] X. Zhang, Q. Liu, J-W. Luo, A. J. Freeman, and A. Zunger. Hidden spin polarization in inversion-symmetric bulk crystals. Nature Physics, 10:387, 2014. 
[34] G. Bergmann. Amorphous metals and their superconductivity. Physics Reports, 27(4):159, 1976.

[35] C. C. Tsuei. Amorphous superconductors, pages 735-756. Springer US, Boston, MA, 1981. ISBN 978-1-4757-0037-4.

[36] O. Prakash, A. Kumar, A. Thamizhavel, and S. Ramakrishnan. Evidence for bulk superconductivity in pure bismuth single crystals at ambient pressure. Science, $355(6320): 52,2017$.

[37] A. V. Narlikar. Frontiers in superconducting materials. Springer, 2005. ISBN 3-540-24513-8.

[38] J. P. Castellan, B. D. Gaulin, H. A. Dabkowska, A. Nabialek, G. Gu, X. Liu, and Z. Islam. Two- and three-dimensional incommensurate modulation in optimallydoped $\mathrm{Bi}_{2} \mathrm{Sr}_{2} \mathrm{CaCu}_{2} \mathrm{O}_{8+\delta}$. Phys. Rev. B, 73:174505, 2006 .

[39] K. M. Shen, F. Ronning, D. H. Lu, F. Baumberger, N. J. C. Ingle, W. S. Lee, W. Meevasana, Y. Kohsaka, M. Azuma, M. Takano, H. Takagi, and Z. X. Shen. Nodal quasiparticles and antinodal charge ordering in $\mathrm{Ca}_{2-x} \mathrm{Na}_{x} \mathrm{CuO}_{2} \mathrm{Cl}_{2}$. Science, 307(5711):901-904, 2005.

[40] T. Kato, T. Machida, Y. Kamijo, R. Miyashita, and H. Sakata. Spatial correlation between the LDOS modulation and electronic inhomogeneity in $\mathrm{Bi}_{2} \mathrm{Sr}_{2-x} \mathrm{La}_{x} \mathrm{CuO}_{6+\delta}$. Journal of Physics: Conference Series, 150(5):052101, 2009.

[41] K. Chatterjee T. Kondo T. Takeuchi H. Ikuta Y. Wang W. D. Wise, M. C. Boyer and E. W. Hudson. Charge-density-wave origin of cuprate checkerboard visualized by scanning tunnelling microscopy. Nature Physics, 4:696 - 699, 2008.

[42] H. Jang, W.-S. Lee, H. Nojiri, S. Matsuzawa, H. Yasumura, L. Nie, A. V. Maharaj, S. Gerber, Y.-J. Liu, A. Mehta, D. A. Bonn, R. Liang, W. N. Hardy, C. A. Burns, Z. Islam, S. Song, J. Hastings, T. P. Devereaux, Z.-X. Shen, S. A. Kivelson, C.-C. Kao, D. Zhu, and J.-S. Lee. Ideal charge-density-wave order in the high-field state of superconducting YBCO. Proceedings of the National Academy of Sciences, 113 (51):14645-14650, 2016.

[43] K. Chatterjee M. Yi T. Kondo T. Takeuchi H. Ikuta E. W. Hudson M. C. Boyer, W. D. Wise. Imaging the two gaps of the high-temperature superconductor $\mathrm{Bi}_{2} \mathrm{Sr}_{2} \mathrm{CuO}_{6+x}$. Nature Physics, 3:802, 2007.

[44] H-H. Wen, G. Mu, H. Luo, H. Yang, L. Shan, C. Ren, P. Cheng, J. Yan, and L. Fang. Specific-heat measurement of a residual superconducting state in the 
normal state of underdoped $\mathrm{Bi}_{2} \mathrm{Sr}_{2-x} \mathrm{La}_{x} \mathrm{CuO}_{6+\delta}$ cuprate superconductors. Phys. Rev. Lett., 103:067002, 2009.

[45] T. Ekino M. S. Li H. Szymczak A. M. Gabovich, A. I. Voitenko and M. Pkaa. Competition of superconductivity and charge density waves in cuprates: recent evidence and interpretation. Advances in Condensed Matter Physics, 2010:681070, 2010 .

[46] M. B. Shalom, M. Sachs, D. Rakhmilevitch, A. Palevski, and Y. Dagan. Tuning spin-orbit coupling and superconductivity at the $\mathrm{SrTiO}_{3} / \mathrm{LaAlO}_{3}$ interface: a magnetotransport study. Phys. Rev. Lett., 104:126802, 2010.

[47] J. Ranninger R. Micnas and S. Robaszkiewicz. Superconductivity in narrow-band systems with local nonretarded attractive interactions. Rev. Mod. Phys., 62(1): 113, 1990.

[48] H. Woo, P. Dai, S. M. Hayden, H. A. Mook, T. Dahm, D. J. Scalapino, T. G. Perring, and F. Doğan. Magnetic energy change available to superconducting condensation in optimally doped $\mathrm{YBa}_{2} \mathrm{Cu}_{3} \mathrm{O}_{6} .95$. Nat. phys., 2:600, 2006.

[49] T. Dahm, V. Hinkov, S. V. Borisenko, A. A. Kordyuk, V. B. Zabolotnyy, J. Fink, B. Büchner, D. J. Scalapino, W. Hanke, and B. Keimer. Strength of the spinfluctuation-mediated pairing interaction in a high-temperature superconductor. Nat. phys., 5:217, 2009.

[50] R. Zhi-An, L. Wei, Y. Jie, Y. Wei, S. Xiao-Li, Zheng-Cai, C. Guang-Can, D. XiaoLi, S. Li-Ling, Z. Fang, and Z. Zhong-Xian. Superconductivity at $55 \mathrm{~K}$ in ironbased F-doped layered quaternary compound $\mathrm{Sm}\left[\mathrm{O}_{1-x} F_{x}\right]$ FeAs. Chinese Physics Letters, 25(6):2215, 2008.

[51] Y. Kamihara, H. Hiramatsu, M. Hirano, R. Kawamura, H. Yanagi, T. Kamiya, and H. Hosono. Iron-based layered superconductor: LaOFeP. Journal of the American Chemical Society, 128(31):10012-10013, 2006.

[52] K. Arii Y. Kamihara M. Hirano H. Hosono H. Takahashi, K. Igawa. Superconductivity at $43 \mathrm{~K}$ in an iron-based layered compound $\mathrm{LaO}_{1-x} \mathrm{~F}_{x} \mathrm{FeAs}$. Nature, 453: 376-378, 2008.

[53] C-H. Lee, A. Iyo, H. Eisaki, H. Kito, M. T. Fernandez-Diaz, T. Ito, K. Kihou, H. Matsuhata, M. Braden, and K. Yamada. Effect of structural parameters on superconductivity in fluorine-free $\mathrm{LnFeAsO}_{1-y}(\mathrm{Ln}=\mathrm{La}, \mathrm{Nd})$. J Phy. Soc. Jap., 77(8):083704, 2008. 
[54] Y. Mizuguchi, Y. Hara, K. Deguchi, S. Tsuda, T Yamaguchi, K. Takeda, H. Kotegawa, H. Tou, and Y. Takano. Anion height dependence of $\mathrm{T}_{c}$ for the Fe-based superconductor. Supercond. Sci. Technol., 23:054013, 2010.

[55] Y. Mizuguchi. Discovery of $\mathrm{BiS}_{2}$-based superconductor and material design concept. Condensed Matter, 2(1):6, 2017.

[56] Y. Mizuguchi, A. Nishida, A. Omachi, and O. Miura. Thermoelectric properties of new Bi-chalcogenide layered compounds. Cogent Physics, 3(1), 2016.

[57] A. Miura, Y. Mizuguchi, T. Sugawara, Y. Wang, T. Takei, N. Kumada, E. Magome, C. Moriyoshi, Y. Kuroiwa, O. Miura, and K. Tadanaga. Structural difference in superconductive and nonsuperconductive $\mathrm{BiS}$ planes within $\mathrm{Bi}_{4} \mathrm{O}_{4} \mathrm{Bi}_{2} \mathrm{~S}_{4}$ blocks. Inorganic Chemistry, 54(21):10462-10467, 2015.

[58] H. Takatsu, Y. Mizuguchi, H. Izawa, O. Miura, and H. Kadowaki. Bulk superconductivity in $\mathrm{Bi}_{4} \mathrm{O}_{4} \mathrm{~S}_{3}$ revealed by specific heat measurement. J Phys. Soc. Jpn., 81(12):125002, 2012.

[59] Y. Mizuguchi. Review of superconductivity in $\mathrm{BiS}_{2}$-based layered materials. Journal of Physics and Chemistry of Solids, 84:34, 2015.

[60] V.P.S. Awana, A. Kumar, R. Jha, S. K. Singh, A. Pal, Shruti, J. Saha, and S. Patnaik. Appearance of superconductivity in layered $\mathrm{LaO}_{0.5} \mathrm{~F}_{0.5} \mathrm{BiS}_{2}$. Solid State Communications, 157:21, 2013.

[61] I. R. Shein and A. L. Ivanovskii. Electronic band structure and Fermi surface for new layered superconductor $\mathrm{LaO}_{0.5} \mathrm{~F}_{0.5} \mathrm{BiS}_{2}$ in comparison with parent phase $\mathrm{LaOBiS}_{2}$ from first principles. JETP Letters, 96(12):769, 2013.

[62] J. Xing, S. L., X. Ding, H. Yang, and H-H. Wen. Superconductivity appears in the vicinity of semiconducting-like behavior in $\mathrm{CeO}_{1-x} \mathrm{~F}_{x} \mathrm{BiS}_{2}$. Phys. Rev. B, 86: $214518,2012$.

[63] R. Jha and V. P. S. Awana. Superconducting properties of $\mathrm{BiS}_{2}$-based superconductor $\mathrm{NdO}_{1-x} \mathrm{~F}_{x} \mathrm{BiS}_{2}$ ( $x=0$ to 0.9). Materials Research Express, 1(1):016002, 2014.

[64] J. Kajitani, K. Deguchi, A. Omachi, T. Hiroi, Y. Takano, H. Takatsu, H. Kadowaki, O. Miura, and Y. Mizuguchi. Correlation between crystal structure and superconductivity in $\mathrm{LaO}_{0.5} \mathrm{~F}_{0.5} \mathrm{BiS}_{2}$. Solid State Communications, 181:1, 2014.

[65] C. T. Wolowiec, B. D. White, I. Jeon, D. Yazici, K. Huang, and M. B. Maple. Enhancement of superconductivity near the pressure-induced semiconductormetal 
transition in the $\mathrm{BiS}_{2}$-based superconductors $\mathrm{LnO}_{0.5} \mathrm{~F}_{0.5} \mathrm{BiS}_{2}(\mathrm{Ln}=\mathrm{La}, \mathrm{Ce}, \mathrm{Pr}$, Nd). Journal of Physics: Condensed Matter, 25(42):422201, 2013.

[66] C. T. Wolowiec, D. Yazici, B. D. White, K. Huang, and M. B. Maple. Pressureinduced enhancement of superconductivity and suppression of semiconducting behavior in $L n \mathrm{O}_{0.5} \mathrm{~F}_{0.5} \mathrm{BiS}_{2}(L n=\mathrm{La}, \mathrm{Ce})$ compounds. Phys. Rev. B, 88:064503, 2013.

[67] H. Kotegawa, Y. Tomita, H. Tou, H. Izawa, Y. Mizuguchi, O. Miura, S. Demura, K. Deguchi, and Y. Takano. Pressure study of BiS2-based superconductors $\mathrm{Bi}_{4} \mathrm{O}_{4} \mathrm{~S}_{3}$ and $\mathrm{La}(\mathrm{O}, \mathrm{F}) \mathrm{BiS}_{2}$. J Phys. Soc. Jpn., 81(10):103702, 2012.

[68] T. Tomita, M. Ebata, H. Soeda, H. Takahashi, H. Fujihisa, Y. Gotoh, Y. Mizuguchi, H. Izawa, O. Miura, S. Demura, K. Deguchi, and Y. Takano. Pressure-induced enhancement of superconductivity and structural transition in BiS2-layered $\mathrm{LaO}_{1-x} \mathrm{~F}_{x} \mathrm{BiS}_{2}$. Journal of the Physical Society of Japan, 83(6): 063704, 2014.

[69] H. Kotegawa, Y. Tomita, H. Tou, H. Izawa, Y. Mizuguchi, O. Miura, S. Demura, K. Deguchi, and Y. Takano. Pressure study of $\mathrm{BiS}_{2}$-based superconductors $\mathrm{Bi}_{4} \mathrm{O}_{4} \mathrm{~S}_{3}$ and $\mathrm{La}(\mathrm{O}, \mathrm{F}) \mathrm{BiS}_{2}$. Journal of the Physical Society of Japan, 81(10): $103702,2012$.

[70] R. Akashi M. Ochi and K. Kuroki. Strong bilayer coupling induced by the symmetry breaking in the monoclinic phase of $\mathrm{BiS}_{2}$-based superconductors. Journal of the Physical Society of Japan, 85(9):094705, 2016.

[71] Q. Liu, X. Zhang, and A. Zunger. Polytypism in $\mathrm{LaOBiS}_{2}$-type compounds based on different three-dimensional stacking sequences of two-dimensional $\mathrm{BiS}_{2}$ layers. Phys. Rev. B, 93:174119, 2016.

[72] C. Y. Guo, Y. Chen, M. Smidman, S. A. Chen, W. B. Jiang, H. F. Zhai, Y. F. Wang, G. H. Cao, J. M. Chen, X. Lu, and H. Q. Yuan. Evidence for two distinct superconducting phases in $\mathrm{EuBiS}_{2} \mathrm{~F}$ under pressure. Phys. Rev. B, 91:214512, 2015.

[73] G. S. Thakur, R. Jha, Z. Haque, V. P. S. Awana, L. C. Gupta, and A. K. Ganguli. Pressure enhanced superconductivity at $10 \mathrm{~K}$ in La doped $\mathrm{EuBiS}_{2}$ F. Superconductor Science and Technology, 28(11):115010, 2015.

[74] J. Liu, S. Li, Y. Li, X. Zhu, and H-H. Wen. Pressure-tuned enhancement of superconductivity and change of ground state properties in $\mathrm{LaO}_{0.5} \mathrm{~F}_{0.5} \mathrm{BiSe}_{2}$ single crystals. Phys. Rev. B, 90:094507, 2014. 
[75] M. Fujioka, M. Tanaka, S. J. Denholme, T. Yamaki, H. Takeya, T. Yamaguchi, and Y. Takano. Pressure-induced phase transition for single-crystalline $\mathrm{LaO}_{0.5} \mathrm{~F}_{0.5} \mathrm{BiSe}_{2}$. EPL (Europhysics Letters), 108(4):47007, 2014.

[76] Y. Luo, H-F. Zhai, P. Zhang, Z-A. Xu, G-H. Cao, and J. D. Thompson. Pressureenhanced superconductivity in $\mathrm{Eu}_{3} \mathrm{Bi}_{2} \mathrm{~S}_{4} \mathrm{~F}_{4}$. Phys. Rev. B, 90:220510, 2014.

[77] C. Morice, R. Akashi, T. Koretsune, S. S. Saxena, and R. Arita. Weak phononmediated pairing in $\mathrm{BiS}_{2}$ superconductor from first principles. Phys. Rev. B, 95: $180505,2017$.

[78] M. Nagao, S. Demura, K. Deguchi, A. Miura, S. Watauchi, T. Takei, Y. Takano, N. Kumada, and I. Tanaka. Structural analysis and superconducting properties of F-substituted $\mathrm{NdOBiS}_{2}$ single crystals. J Phy. Soc. Jap., 82:113701, 2013.

[79] M. Mittag, M. Rosenberg, D. Peligrad, R. Wernhardt, V. A. M. Brabers, J. H. P. M. Emmen, and D. Hu. A study of the reversible and irreversible magnetic properties of a Bi-2212 single crystal in high magnetic fields. Superconductor Science and Technology, 7(4):214, 1994.

[80] S.G. Kozlova, S.P. Gabuda, M.R. Ryzhikov, and V.A. Slepkov. Topological characteristics of electron density and magnetic anisotropy in $\mathrm{MgB}_{2}$. Computational and Theoretical Chemistry, 1066:100 - 103, 2015.

[81] L. Aslamasov and L. Larkin. Effect of fluctuations on the properties of a superconductor above the critical temperature. Soviet Physics - Solid State, 10(4):875, 1968.

[82] I. M. Khalatnikov and V. P. Mineev. 30 Years of the Landau Institute - selected papers. World Scientific, 1996. ISBN 981-02-2253-X.

[83] P. K. Biswas, A. Amato, C. Baines, R. Khasanov, H. Luetkens, Hechang Lei, C. Petrovic, and E. Morenzoni. Low superfluid density and possible multigap superconductivity in the $\mathrm{BiS}_{2}$-based layered superconductor $\mathrm{Bi}_{4} \mathrm{O}_{4} \mathrm{~S}_{3}$. Phys. Rev. $B, 88: 224515,2013$.

[84] Y. Onishi and K. Miyake. Enhanced valence fluctuations caused by f-c coulomb interaction in ce-based heavy electrons: possible origin of pressure-induced enhancement of superconducting transition temperature in $\mathrm{CeCu}_{2} \mathrm{Ge}_{2}$ and related compounds. Journal of the Physical Society of Japan, 69(12):3955-3964, 2000.

[85] C. M. Varma. Missing valence states, diamagnetic insulators, and superconductors. Phys. Rev. Lett., 61:2713-2716, 1988. 
[86] Putti, M., G. d'Agliano, E., Marr, D., Napoli, F., Tassisto, M., Manfrinetti, P., Palenzona, A., Rizzuto, C., and Massidda, S. Electron transport properties of $\mathrm{MgB}_{2}$ in the normal state. Eur. Phys. J. B, 25(4):439-443, 2002.

[87] Y. Yang, W-S. Wang, Y-Y. Xiang, Z-Z. Li, and Q-H. Wang. Triplet pairing and possible weak topological superconductivity in $\mathrm{BiS}_{2}$-based superconductors. Phys. Rev. B, 88:094519, 2013.

[88] H. Usui, K. Suzuki, and K. Kuroki. Minimal electronic models for superconducting BiS $_{2}$ layers. Phys. Rev. B, 86:220501, 2012.

[89] T. Zhou and Z. D. Wang. Probing the superconducting pairing symmetry from spin excitations in $\mathrm{BiS}_{2}$ based superconductors. Journal of Superconductivity and Novel Magnetism, 26(8):2735-2740, 2013.

[90] Yi Liang, Xianxin Wu, Wei-Feng Tsai, and Jiangping Hu. Pairing symmetry in layered $\mathrm{BiS}_{2}$ compounds driven by electron-electron correlation. Frontiers of Physics, 9(2):194, 2014.

[91] J. Chadwick. Possible existence of a neutron. Nature, 129(3252):312, 1932.

[92] G. L. Squires. Introduction to the theory of thermal neutron scattering. Cambridge, 1978. ISBN 978-1-107-64406-9.

[93] L. V. Hove. Correlations in space and time and Born approximation scattering in systems of interacting particles. Phys. Rev., 95(1):249, 1954.

[94] S. W. Lovesey. Theory of neutron scattering from condensed matter volume 1. Clarendon Press. Oxford, 1984. ISBN 0-19-852017-4.

[95] T. Egami and S.J.L. Billinge. Underneath the Bragg peaks structural analysis of complex materials. Pergamon, 2003. ISBN 0080426980.

[96] B.H. Toby and T. Egami. Accuracy of pair distribution function analysis applied to crystalline and non-crystalline materials. Acta Cryst., A48:336, 1992.

[97] S. J. L. Billinge and M. G. Kanatzidis. Beyond crystallography: the study of disorder, nanocrystallinity and crystallographically challenged materials with pair distribution functions. Chem. Commun., 7:749, 2004.

[98] P. F. Peterson, E. S. Božin, T. Proffen, and S. J. L. Billinge. Improved measures of quality for the atomic pair distribution function. Journal of Applied Crystallography, 36(1):53, Feb 2003. 
[99] G. Beni and P. M. Platzmanick. Temperature and polarization dependence of extended x-ray absorption fine-structure spectra. Phys. Rev. B, 14:1514, 1976.

[100] M.J. Graf S.J.L. Billinge I.-K. Jeong, R.H. Heffner. Lattice dynamics and correlated atomic motion from the pair distribution function. Phys. Rev. B, 67:104301, 2003.

[101] K. C. Holmes G. Rosenbaum and J. Witz. Synchrotron radiation as a source for X-ray diffraction. Nature, 230:434, 1971.

[102] A. Guinier. X-ray diffraction. W. H. Freeman and Company, 1963. ISBN 62-13298.

[103] High resolution powder diffractometer, D1B. URL https://www.ill.eu/ instruments-support/instruments-groups/instruments/d1b/description/ instrument-layout/.

[104] F. Fernandez-Alonso and D. L. Price. Neutron scattering fundamentals. Elsevier, 2013. ISBN 978-0-12-398374-9.

[105] The four circle single crystal diffractometer. URL www.ems.psu.edu/ ryba/ coursework/530/four\%20circle.ppt.

[106] B. H. Toby. Expgui, a graphical user interface for gsas. J. Appl. Cryst., 34:210, 2001.

[107] A.C. Larson and R.B.V. Dreele. General structure analysis system (GSAS). Los Alamos National Laboratory Report LAUR, page 86, 1994.

[108] B. H. Toby. R factors in rietveld analysis: how good is good enough? Powder Diffraction, 21(1):67, 2006.

[109] G. Placzek. The scattering of neutrons by systems of heavy nuclei. Phys. Rev., $86: 377,1952$.

[110] G. M. Sheldrick. A short history of SHELX. Acta Crystallographica Section A, 64 (1):112, 2008.

[111] S. Demura H. Hara T. Watanabe S. J. Denholme M. Fujioka H. Okazaki T. Ozaki H. Takeya T. Yamaguchi O. Miura K. Deguchi, Y. Mizuguchi and Y. Takano. Evolution of superconductivity in $\mathrm{LaO}_{1-x} \mathrm{~F}_{x} \mathrm{BiS}_{2}$ prepared by high-pressure technique. Europhysics Letters, 101(1):17004, 2013.

[112] A. Miura, M. Nagao, T. Takei, S. Watauchi, I. Tanaka, and N. Kumada. Crystal structures of $\mathrm{LaO}_{1-x} \mathrm{~F}_{x} \mathrm{BiS}_{2}(x \sim 0.23,0.46)$ : effect of $\mathrm{F}$ doping on distortion of BiS plane. Journal of Solid State Chemistry, 212:213, 2014. 
[113] J. Lee, M. B. Stone, A. Huq, T. Yildirim, G. Ehlers, Y. Mizuguchi, O. Miura, Y. Takano, K. Deguchi, S. Demura, and S.-H. Lee. Crystal structure, lattice vibrations, and superconductivity of $\mathrm{LaO}_{1-x} \mathrm{~F}_{x} \mathrm{BiS}_{2}$. Phys. Rev. B, 87:205134, 2013.

[114] A. Athauda, J. Yang, B. Li, Y. Mizuguchi, S-H. Lee, and D. Louca. The crystal structure of superconducting $\mathrm{LaO}_{1-x} \mathrm{~F}_{x} \mathrm{BiS}_{2}$. Journal of Superconductivity and Novel Magnetism, 28(4):1255, 2015.

[115] T. Egami, Y. Petrov, and D. Louca. Lattice effects on charge localization in cuprates. Journal of Superconductivity, 13(5):709, 2000.

[116] D. Yazici, I. Jeon, B.D. White, and M.B. Maple. Superconductivity in layered $\mathrm{BiS}_{2}$-based compounds. Physica C: Superconductivity and its Applications, 514: 218, 2015. Superconducting materials: conventional, unconventional and undetermined.

[117] K. Huang. X-ray reflexions from dilute solid solutions. Proceedings of the Royal Society of London A: Mathematical, Physical and Engineering Sciences, 190(1020): $102,1947$.

[118] J. O. Sofo, A. A. Aligia, and M. D. N. Regueiro. Effect of the local charge disproportionation on the electronic structure of $\mathrm{BaPb}_{1-\mathrm{x}} \mathrm{Bi}_{\mathrm{x}} \mathrm{O}_{3}$. Phys. Rev. B, 40:6955, 1989.

[119] S. Ishiwata, M. Azuma, M. Hanawa, Y. Moritomo, Y. Ohishi, K. Kato, M. Takata, E. Nishibori, M. Sakata, I. Terasaki, and M. Takano. Pressure/temperature/substitution-induced melting of A-site charge disproportionation in $\mathrm{Bi}_{1-x} \mathrm{La}_{x} \mathrm{NiO}_{3}(0 \leqslant x \leqslant 0.5)$. Phys. Rev. B, 72:045104, 2005.

[120] M. Gupta and R. P. Gupta. Role of local coordination geometry in charge transfer in $\mathrm{YBa}_{2} \mathrm{Cu}_{3} \mathrm{O}_{7-\delta}$ superconductors. J. Phys. III France, 2:175, 1992.

[121] S. Demura, Y. Mizuguchi, K. Deguchi, H. Okazaki, H. Hara, T. Watanabe, S. J. Denholme, M. Fujioka, T. Ozaki, H. Fujihisa, Y. Gotoh, O. Miura, T. Yamaguchi, H. Takeya, and Y. Takano. New member of $\mathrm{BiS}_{2}$-based superconductor $\mathrm{NdO}_{1-x} \mathrm{~F}_{x} \mathrm{BiS}_{2}$. Journal of the Physical Society of Japan, 82(3):033708, 2013.

[122] J. Kajitani T. Hiroi O. Miura K. Tadanaga N. Kumada E. Magome C. Moriyoshi Y. Kuroiwa Y. Mizuguchi, A. Miura. In-plane chemical pressure essential for superconductivity in $\mathrm{BiCh}_{2}$-based (Ch: S, Se) layered structure. Scientific Reports, $5: 14968,2015$. 
[123] Y. Mizuguchi. Recent advances in layered metal chalcogenides as superconductors and thermoelectric materials: Fe-based and Bi-based chalcogenides. The Chemical Record, 16(2):633, 2016.

[124] G. S. Thakur, G. K. Selvan, Z. Haque, L. C. Gupta, S. L. Samal, S. Arumugam, and A. K. Ganguli. Synthesis and properties of $\mathrm{SmO}_{0.5} \mathrm{~F}_{0.5} \mathrm{BiS}_{2}$ and enhancement in $\mathrm{T}_{c}$ in $\mathrm{La}_{1-y} \mathrm{Sm}_{y} \mathrm{O}_{0.5} \mathrm{~F}_{0.5} \mathrm{BiS}_{2}$. Inorganic Chemistry, 54(3):1076-1081, 2015.

[125] Omachi A., Hiroi T., Kajitani J., Miura O., and Mizuguchi Y. Importance of uniaxial compression for the appearance of superconductivity in $\mathrm{NdO}_{1-x} \mathrm{~F}_{x} \mathrm{BiS}_{2}$. Journal of Physics: Conference Series, 507(1):012033, 2014.

[126] J. Neuefeind, M. Feygenson, J. Carruth, R. Hoffmann, and K. K. Chipley. The nanoscale ordered materials diffractometer nomad at the spallation neutron source sns. Nuclear Instruments and Methods in Physics Research Section B: Beam Interactions with Materials and Atoms, 287:68, 2012.

[127] A. Athauda, J. Yang, S-H. Lee, Y. Mizuguchi, K. Deguchi, Y. Takano, O. Miura, and D. Louca. In-plane charge fluctuations in bismuth-sulfide superconductors. Phys. Rev. B, 91:144112, 2015.

[128] A. Athauda, C. Hoffmann, S. Aswartham, J. Terzic, G. Cao, X. Zhu, Y. Ren, and D. Louca. Ferro-Lattice-Distortions and Charge Fluctuations in Superconducting $\mathrm{LaO}_{1-x} \mathrm{~F}_{x} \mathrm{BiS}_{2}$. Journal of the Physical Society of Japan, 86(5):054701, 2017.

[129] T. Muranaka Y. Zenitani J. Akimitsu J. Nagamatsu, N. Nakagawa. Superconductivity at $39 \mathrm{~K}$ in magnesium diboride. Nature, 410:63, 2001.

[130] J. Orenstein and A. J. Millis. Advances in the physics of high-temperature superconductivity. 288(5465):468-474, 2000.

[131] H. Lei, K. Wang, M. Abeykoon, E. S. Bozin, and C. Petrovic. New layered fluorosulfide $\mathrm{SrFBiS}_{2}$. Inorganic Chemistry, 52(18):10685, 2013.

[132] International tables for crystallography volume a: space-group symmetry. URL http://it.iucr.org/A/.

[133] X. Zhou, Q. Liu, J. A. Waugh, H. Li, T. Nummy, X. Zhang, X. Zhu, G. Cao, A. Zunger, and D. S. Dessau. Predicted electronic markers for polytypes of $\mathrm{LaOBiS}_{2}$ examined via angle-resolved photoemission spectroscopy. Phys. Rev. $B, 95: 075118,2017$.

[134] R. Sagayama, H. Sagayama, R. Kumai, Y. Murakami, T. Asano, J. Kajitani, R. Higashinaka, T. D. Matsuda, and Y. Aoki. Symmetry lowering in $\mathrm{LaOBiS}_{2}$ : a 
mother material for $\mathrm{BiS}_{2}$-based layered superconductors. Journal of the Physical Society of Japan, 84(12):123703, 2015.

[135] J-H. Chu, H-H. Kuo, J. G. Analytis, and I. R. Fisher. Divergent nematic susceptibility in an iron arsenide superconductor. Science, 337(6095):710, 2012.

[136] M. Shayegan, K. Karrai, Y. P. Shkolnikov, K. Vakili, E. P. De Poortere, and S. Manus. Low-temperature, in situ tunable, uniaxial stress measurements in semiconductors using a piezoelectric actuator. Applied Physics Letters, 83(25): $5235,2003$.

[137] A. Athauda, Y. Mizuguchi, M. Nagao, J. Neuefeind, and D. Louca. Charge Fluctuations in the $\mathrm{NdO}_{1-x} \mathrm{~F}_{x} \mathrm{BiS}_{2}$ Superconductors. Accepted to Journal of the Physical Society of Japan, 2017.

[138] S. Salem-Sugui, E. E. Alp, S. M. Mini, M. Ramanathan, J. C. Campuzano, G. Jennings, M. Faiz, S. Pei, B. Dabrowski, Y. Zheng, D. R. Richards, and D. G. Hinks. Determination of the local structure in $\mathrm{Ba}_{1-x} \mathrm{~K}_{x} \mathrm{BiO}_{3}$ by x-ray-absorption spectroscopy. Phys. Rev. B, 43:5511, 1991.

[139] I. I. Mazin, A. I. Liechtenstein, and O. V. Ivanov. Breathing instability and disproportionation of $\mathrm{Bi} 4$ ions in $\mathrm{BaBiO}_{3}$. Ferroelectrics, 164(1):169, 1995.

[140] L. F. Mattheiss, E. M. Gyorgy, and D. W. Johnson. Superconductivity above 20 K in the Ba-K-Bi-O system. Phys. Rev. B, 37:3745-3746, 1988.

[141] S. M. Heald, D. DiMarzio, M. Croft, M. S. Hegde, S. Li, and M. Greenblatt. Xray-absorption study of charge-density ordering in $\left(\mathrm{Ba}_{1-\mathrm{x}} \mathrm{K}_{\mathrm{x}}\right) \mathrm{BiO}_{3}$. Phys. Rev. B, 40:8828-8833, 1989.

[142] L. A. Olsen, J. Lpez-Solano, A. Garca, T. Balic Zunic, and E. Makovicky. Dependence of the lone pair of bismuth on coordination environment and pressure: an ab initio study on $\mathrm{Cu}_{4} \mathrm{Bi}_{5} \mathrm{~S}_{10}$ and $\mathrm{Bi}_{2} \mathrm{~S}_{3}$. Journal of Solid State Chemistry, 183(9): 2133-2143, 2010. 